PNL-6824

UC-11, 41

$5 \mathrm{C}$

\title{
Borehole Summary Report \\ for Five Ground-Water \\ Monitoring Wells Constructed \\ in the 1100 Area
}

R.W. Bryce

S.M. Goodwin

May 1989

Prepared for the U.S. Department of Energy under Contract DE-AC06-76RLO 1830

Pacific Northwest Laboratory

Operated for the U.S. Department of Energy

by Battelle Memorial Institute 


\section{DISCLAIMER}

This report was prepared as an account of work sponsored by an agency of the United States Government. Neither the United States Government nor any agency thereof, nor Battelle Memorial Institute, nor any or their employees, makes any warranty, expressed or implied, or assumes any legal liability or responsibility for the accuracy, completeness, or usefulness of any information, apparatus, product, or process disclosed, or represents that its use would not infringe privately owned rights. Reference herein to any specific commercial product, process, or service by trade name, trademark, manufacturer, or otherwise does not necessarily constitute or imply its endorsement, recommendation, or favoring by the United States Government or any agency thereof, or BattelleMemorial Institute. The views and opinions of authors expressed herein do not necessarily state or reflect those of the United States Government or any agency thereof.

\section{PACIFIC NORTHWEST LABORATORY operated by \\ BATTELLE MEMORIAL INSTITUTE for the UNITED STATES DEPARTMENT OF ENERGY under Contract DE-AC06-76RLO 1830}

\begin{tabular}{|c|c|}
\hline \multirow{2}{*}{\multicolumn{2}{|c|}{ Printed in the United States of America }} \\
\hline \multirow{2}{*}{\multicolumn{2}{|c|}{$\begin{array}{c}\text { Available from } \\
\text { National Technical Information Service }\end{array}$}} \\
\hline & \\
\hline \multicolumn{2}{|c|}{ United States Department of Commerce } \\
\hline \multicolumn{2}{|c|}{5285 Port Royal Road } \\
\hline \multicolumn{2}{|c|}{ Springfield, Virginia 22161} \\
\hline \multirow{2}{*}{\multicolumn{2}{|c|}{$\begin{array}{l}\text { NTIS Price Codes } \\
\text { Microfiche A01 }\end{array}$}} \\
\hline & \\
\hline \multicolumn{2}{|c|}{ Printed Copy } \\
\hline & Price \\
\hline Pages & Codes \\
\hline 001-025 & A02 \\
\hline 026-050 & A03 \\
\hline $051-075$ & A04 \\
\hline 076-100 & A05 \\
\hline $101-125$ & A06 \\
\hline $126-150$ & $\mathrm{~A} 07$ \\
\hline $151-175$ & A08 \\
\hline $176-200$ & A09 \\
\hline $201-225$ & A10 \\
\hline $226-250$ & All \\
\hline 251-275 & $\mathrm{A} 12$ \\
\hline $276-300$ & $\mathrm{~A} 13$ \\
\hline
\end{tabular}


BOREHOLE SUMMARY REPORT FOR FIVE GROUND-WATER MONITORING WELS CONSTRUCTED IN THE 1100 AREA

R. W. Bryce

S. M. Goodwin

May 1989

Prepared for the U.S. Department of Energy under Contract DE-AC06-76RLO 1830

Pacific Northwest Laboratory

Richland, Washington 99352 


\section{SUMMARY}

This report contains the data collected during the installation and initial sampling of five ground-water monitoring wells between the 1100 Area and Richland City water supply wells. The five wells were installed to provide for early detection of contaminants and to provide data that may be used in making decisions on the management of the North Richland Well Field and recharge basins. 


\section{CONTENTS}

SUMMARY ......................... $i$. . . .

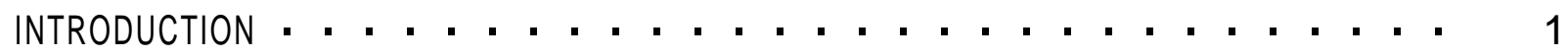

RESULTS ............................... 7

WELL $1 \ldots \ldots \ldots \ldots$

WELL $2 \ldots \ldots \ldots$

WELL $3 \ldots \ldots \ldots . \ldots \ldots$

WELL $4 \ldots \ldots \ldots \ldots \ldots$

WELL $5 \ldots \ldots \ldots \ldots \ldots \ldots$

RESULTS OF GROUND-WATER SAMPLE ANALYSIS . . . . . . . . 13

REFERENCES ........................... 15

APPENDIX A - GEOPHYSICAL AND GEOLOGIC LOGS FOR WELL $1 \ldots \ldots$. . . . . A.I

APPENDIX B - GEOPHYSICAL AND GEOLOGIC LOGS FOR WELL $2 \ldots \ldots$. . . . . B.I

APPENDIX C - GEOPHYSICAL AND GEOLOGIC LOGS FOR WELL $3 \ldots \ldots$. . . . .

APPENDIX D - GEOPHYSICAL AND GEOLOGIC LOGS FOR WELL $4 \ldots \ldots$. . . . . .

APPENDIX E - GEOPHYSICAL AND GEOLOGIC LOGS FOR WELL $5 \ldots$. . . . . E.I

APPENDIX F - WELL SURVEY DATA . . . . . . . . . . F.I

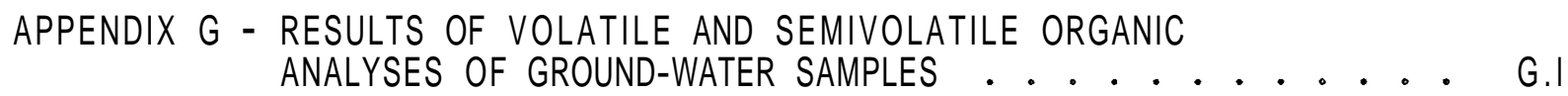

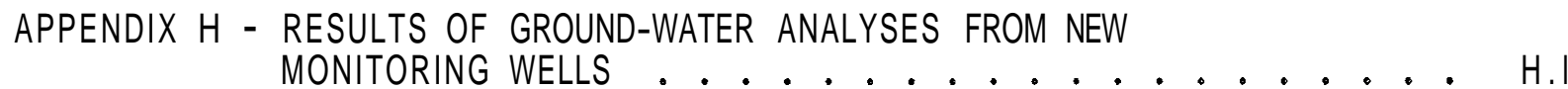


The Hanford Site, operated for the U.S. Department of Energy (DOE) by Westinghouse Hanford Company, consists of several "areas" that accommodate various activities. The 1100 Area, one of the areas near the city of Richland, has been used for maintenance activities. Waste disposal sites in the 1100 Area received unknown quantities of liquid and solid waste between 1950 and 1985. While the types and quantities of waste are not documented, they are known to include spent battery acid, antifreeze, used motor oils, solvents, degreasers, paints, and paint thinners. These disposal sites are in proximity to wells that supply water to Richland. Five monitoring wells were installed between the waste disposal sites and the wells supplying water to Richland to detect contaminants in the ground water, should they exist, before they reach the water supply wells. Background information on the waste sites and water supply wells and a plan for installation of the new wells are presented in Bryce (1989). This document presents the data gathered during the construction of the monitoring wells and the analytical results of the initial sampling of ground water from the wells.

The water supply wells of concern include the Duke Wells, the North Richland Well Field, and well 3000D-5 (Figure 1). The locations of the five new monitoring we11s are shown in Figure 2. We11s 1 and 3 are located between the North Richland Well Field and two waste disposal sites: the Battery Acid Pit and the site of the exhumed waste antifreeze storage tank. The Battery Acid Pit received an estimated 15,000 gal of battery acid and is considered the site with the highest potential to impact the quality of the ground water. These two monitoring wells are located some distance from' the recharge ponds to increase the chance that representative ground water (i.e., unaffected by the recharge ponds) will be collected. This location will also provide water-level elevation data that will help determine the direction of ground-water flow between the waste sites and the water supply wells. Well 1 will intercept contaminants near the top of the aquifer; well 3 will intercept contaminants that have migrated to a depth near the bottom of the aquifer. 


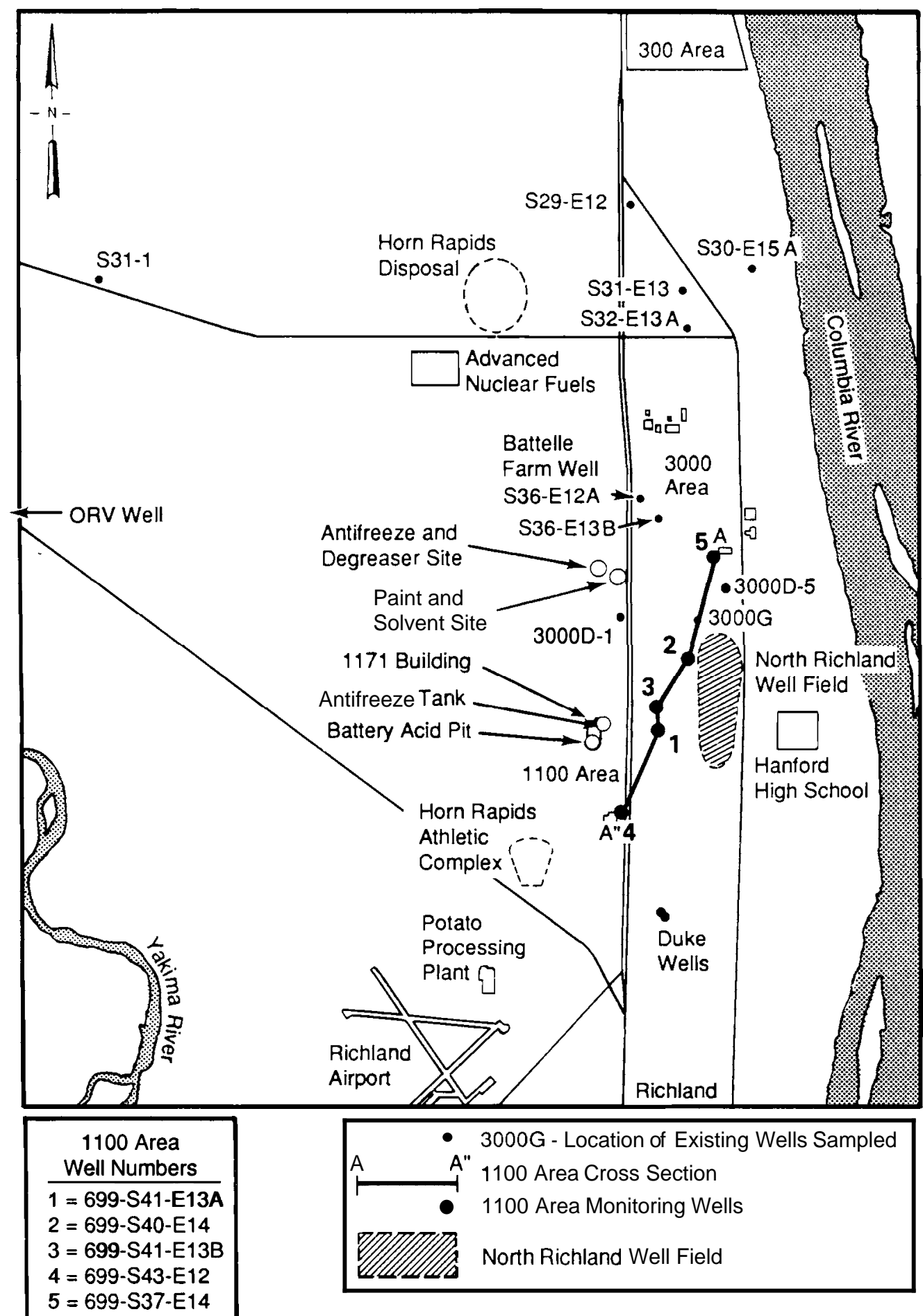

FIGURE 1. Map of the 1100 Area 


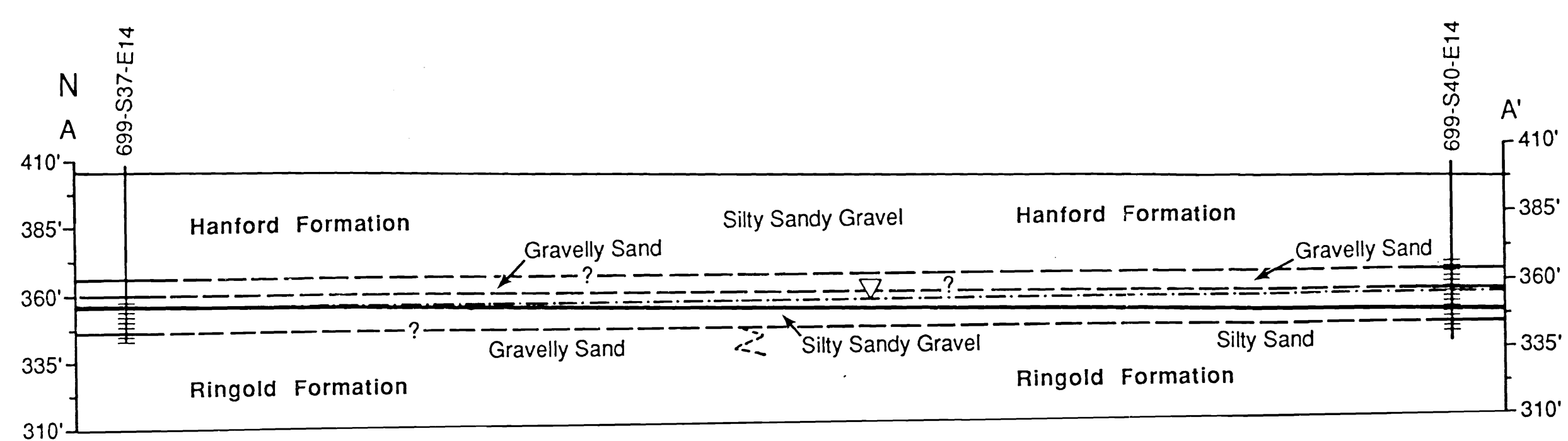

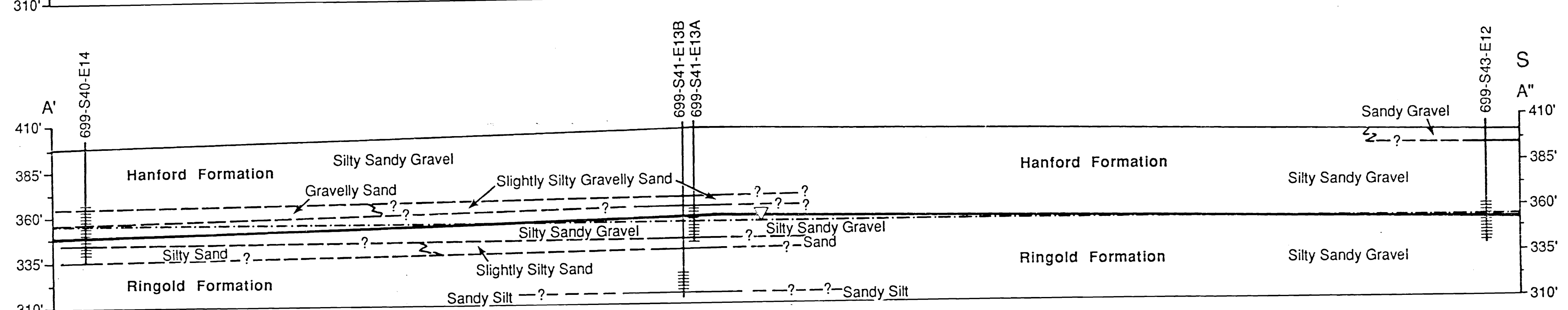

Scale in Feet

$0 \quad 100 \quad 200 \quad 300 \quad 400 \quad 500$

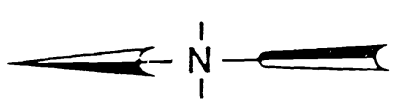

Vertical Exaggeration $=4 x$

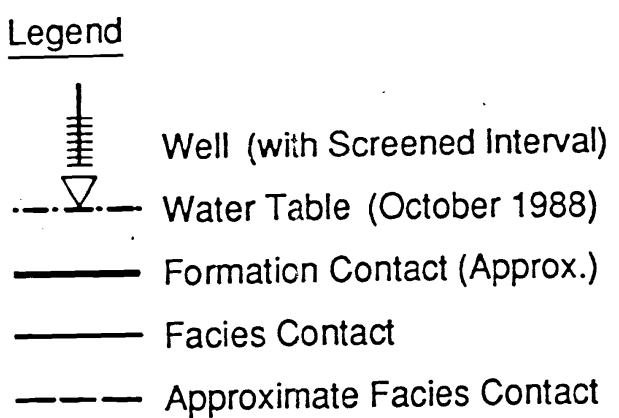

FIGURE 2 Cross Section of the 1100 Area Environmental Monitoring Wells, 1988 
Well 2 is located close to the North Richland Well Field and midway between the Battery Acid Pit and Antifreeze Tank to the south and the Paint and Solvent Site and Antifreeze and Degreaser Site to the north. This well was completed at the top of the aquifer because contaminants, if present, will most likely be found there and the top of the aquifer is tapped by the water supply wells of the North Richland Well Field. This location provides water-level elevation data that may be used to characterize the impact recharge and withdrawal of ground water in the North Richland Well Field has on the local ground-water flow system.

Well 4 is located south of the other wells, between the waste disposal sites and the Duke Wells. During periods when a ground-water mound existed beneath the recharge ponds in the North Richland Well Field, ground water may have flowed south from the vicinity of the Battery Acid Pit and the Antifreeze Tank toward the Duke Wells. A well at this location assisted in determining if flow to the south did occur by allowing the collection of water-table elevation data and ground-water samples for chemical analysis.

Well 5 is located north of the well field to sample ground water in the vicinity of Well 3000D-5. Although the ideal location of this well would be between we11 3000D-5 and the Paint and Solvent Site and Antifreeze and Degreaser Site, the land in this area is not accessible to the DOE. The we11 was therefore drilled to the north of the water supply well of interest, because wells 3000D-1 and 3000-G are south of the well and disposal sites and are available for sample collection there. 


\section{$\underline{\text { RESULTS }}$}

The following sections summarize well construction and ground-water sampling activities at each well site. A geologic cross section summarizing geologic data gathered from these wells is presented in Figure 2. Supporting data, including the Well Completion/Inspection Form, as-built drawing, sample pump installation form, geophysical $\operatorname{logs}$, and geologic $\operatorname{logs}$ for each well, are presented in Appendixes A through E. Well survey data (location and elevation) are presented in Appendix F; results of ground-water analyses are presented in Appendix G.

\section{WELL 1 (699-S41-E13A)}

Drilling at well 699-S41-E13A began October 7, 1988, and reached a total depth of $67 \mathrm{ft}$ below ground surface on October 12, 1988. The sediments encountered were primarily silty sandy gravels and slightly silty gravel ly sands. The Ringold Formation was tentatively identified at $50 \mathrm{ft}$ below ground surface; this assumption was based on changes in drilling rates, soil color, basalt percentages, and increased field reactions to $10 \% \mathrm{HCl}$. Additional laboratory analyses would be necessary to further define the formation contact. Static water level during well completion was $53.25 \mathrm{ft}$ below ground surface.

Well 699-S41-E13A was completed at a depth of $62.8 \mathrm{ft}$ below ground surface. Final well materials include $15.65 \mathrm{ft}$ of 20-slot Johnson Division type 304 stainless steel screen (4 in. dia) and $50 \mathrm{ft}$ of $4 \mathrm{in}$. Johnson Division type 304 stainless steel casing. Factory-welded stainless steel casing centralizers were located 7.15, 27.15, 57.55, and $65.65 \mathrm{ft}$ below land surface. The annular seal consists of a 16-30 mesh Colorado silica sand pack along the screened interval to $3.65 \mathrm{ft}$ above the top of the screen, $6.3 \mathrm{ft}$ of Enviroplug bentonite pellets, (a) $18.83 \mathrm{ft}$ of 8-20 mesh granular bentonite crumbles, $17.07 \mathrm{ft}$ of Portland cement grout, and an air-entrained concrete seal that reaches $1.3 \mathrm{ft}$ below ground surface. The casing was pulled back with the Bucyrus Erie $22 \mathrm{~W}$ cable-tool drill rig that drilled the borehole.

(a) Manufactured by Wyo-Ben, Inc., Bill ings, Montana. 
The estimated volume of water added to the borehole during drilling is $120 \mathrm{gal}$; the estimated volume of water removed during the two-phase development process is $1287 \mathrm{gal}$. Water added to the borehol'e during drilling was obtained from the Richland municipal water supply in the 3000 Area and was stored onsite in 55-gal steel drums. Field monitoring for contamination was performed by geologists and radiation protection technologists from Pacific Northwest Laboratory (PNL). № contamination was detected, so the vadose zone was not sampled for hazardous constituents.

Well development consisted of bail ing the completed well with the cabletool rig and dart bailer until the water was essentially sediment free; continued development was performed with a 1.5-hp submersible pump. The well was considered developed when water samples were consistently below the 5-NTU criteria specified in the Technical Enforcement Guidance Document (EPA 1986); water samples were analyzed with a Hach Portable Turbidimeter. Development water was discharged to the ground after receiving acceptable results from a rapid water analysis sent to U.S. Testing Company, Inc. (UST), on October 12, 1988. No aquifer test was performed. The HydroStar(a) pump was installed on November 3, 1988; this completed construction activities at we11 699-S41-E13A.

\section{WELL 2 (699-S40-E14)}

Drilling at well 699-S40-E14 began October 3, 1988, and reached a total depth of $62.44 \mathrm{ft}$ below ground surface on October 6, 1988. The sediments encountered were primarily silty sandy gravels and gravelly sands. The middle Ringold member was encountered at approximately $52 \mathrm{ft}$ below ground surface; static water level during well completion was $44.43 \mathrm{ft}$ below ground surface.

Well 699-S40-E14 was completed at a depth of $59.5 \mathrm{ft}$ below ground surface. Final well materials include $25.95 \mathrm{ft}$ of 10 -slot Johnson Division type 304 stainless steel screen (4 in. dia) and $35 \mathrm{ft}$ of 4 -in. Johnson

(a) HydroStar is a tradename of Instrumentation Northwest, Inc., Redmond, Washington. 
Division type 304 stainless steel casing. Factory-welded stainless steel casing centralizers were located at $34.5,53.5$, and $59.5 \mathrm{ft}$ below ground surface. The annular seal consists of a 20-40 mesh Colorado silica sand pack along the screened interval to $3.40 \mathrm{ft}$ above the top of the screen, $6.85 \mathrm{ft}$ of Enviroplug bentonite pellets, $3.45 \mathrm{ft}$ of 8-20 mesh granular bentonite crumbles, $16.45 \mathrm{ft}$ of Portland cement grout, and an air-entrained concrete seal that reaches $3.4 \mathrm{ft}$ below ground surface. The casing was pulled back with the Bucyrus Erie $2 \mathrm{~W}$ cable-tool drill rig that drilled the borehole.

The estimated volume of water added to the borehole during drilling is 120 gal ; the estimated volume of water removed during the two-phase development process is $689 \mathrm{gal}$. Water added to the borehole was obtained from the Richland municipal water supply in the 3000 Area and was stored onsite in 55-gal steel drums. Field monitoring for contamination was performed by PNL geologists and radiation protection technologists. $\mathrm{N}$ contamination was detected, so the vadose zone was not sampled for hazardous constituents.

Well development consisted of bailing the completed well with the cabletool rig and dart bailer until the water was essentially sediment free; continued development was performed with a 1.5 -hp submersible pump. The well was considered developed when water samples were consistently below the 5-NTU criteria (EPA 1986); water samples were analyzed with a Hach Portable Turbidimeter. Results from a rapid water analysis sent to UST on October 6, 1988, indicated that the development water could be discharged to the ground; however, because of the proximity of the North Richland Well Field, the development water was contained. $\mathrm{N}$ aquifer test was performed. The HydroStar pump was installed on November 3, 1988; this completed construction activities at well 699-S40-E14.

\section{WELL 3 (699-S41-E13B)}

Drilling at well 699-S41-E13B began October 3, 1988. The well was drilled to a depth of $95 \mathrm{ft}$ below ground surface by October 12, 1988. The sediments encountered were primarily silty sandy gravels with the final $9 \mathrm{ft}$ of drilling in sandy silt and clay. The middle Ringold member was 
encountered at approximately $50 \mathrm{ft}$ below ground surface; static water level during we 11 completion was $52.90 \mathrm{ft}$ below ground surface.

Well 699-S41-E13B was completed at a depth of $86.85 \mathrm{ft}$ below ground surface. Final well materials include $10.3 \mathrm{ft}$ of 20 -slot Johnson Division type 304 stainless steel screen (4 in. dia) and $78 \mathrm{ft}$ of 4 -in. Johnson Division type 304 stainless steel casing. Factory-welded stainless steel casing centralizers were located at 36.55 and $76.55 \mathrm{ft}$ below ground surface. The annular seal consists of a 16-30 mesh Colorado silica sand pack along the screened interval to $4.55 \mathrm{ft}$ above the top of the screen, $28.15 \mathrm{ft}$ of bentonite grout, $25.20 \mathrm{ft}$ of bentonite slurry, $15.85 \mathrm{ft}$ of Portland cement grout, and an air-entrained concrete seal that reaches $2.8 \mathrm{ft}$ below ground surface. The casing was pulled back with the Bucyrus Erie 60L cable-tool drill rig that drilled the borehole.

The estimated volume of water added to the borehole during drilling is $150 \mathrm{gal}$; the estimated volume of water removed during the two-phase development process is 1968 gal. Water added to the borehole was obtained from the Richland municipal water supply in the 3000 Area and was stored onsite in 55-gal steel drums. Field monitoring for contamination was performed by PNL geologists and PNL radiation protection technologists. $\mathrm{N}$ contamination was detected, so the vadose zone was not sampled for hazardous constituents.

Well development consisted of bailing the completed well with the cabletool rig and dart bailer until the water was essentially sediment free; continued development was performed with a 1.5-hp submersible pump. The well was considered developed when water samples were consistently below the 5-NTU criteria (EPA 1986); water samples were analyzed with a Hach Portable Turbidimeter. Development water was discharged to the ground after receiving acceptable results from a rapid water analysis sent to UST on October 12, 1988. No aquifer test was performed. The HydroStar pump was installed on November 3, 1988; this completed construction activities at well $699-$ S41-E13B. 


\section{WELL 4 (699-S43-E12)}

Drilling at well 699-\$43-E12 began October 13, 1988. The well was drilled to a depth of $61.75 \mathrm{ft}$ below ground surface by October 20, 1988. The sediments encountered were primarily silty sandy gravels and sandy gravels. The middle Ringold member was encountered at approximately $50 \mathrm{ft}$ below ground surface; static water level during well completion was $48.20 \mathrm{ft}$ below ground surface.

Well 699-S43-E12 was completed at a depth of $58.0 \mathrm{ft}$ below ground surface. Final well materials include $16.0 \mathrm{ft}$ of 20 -slot Johnson Division type 304 stainless steel screen ( $4 \mathrm{in}$. dia) and $45 \mathrm{ft}$ of 4 -in. Johnson Division type 304 stainless steel casing. Factory-welded stainless steel casing centralizers were located at 22 and $52.6 \mathrm{ft}$ below ground surface. The annular seal consists of a 16-30 mesh Colorado silica sand pack along the screened interval to $4.65 \mathrm{ft}$ above the top of the screen, $5.92 \mathrm{ft}$ of Volclay(a) bentonite pellets, $11.75 \mathrm{ft}$ of 8-20 mesh granular bentonite crumbles, $16.70 \mathrm{ft}$ of Portland cement grout, and an air-entrained concrete seal that reaches $3.0 \mathrm{ft}$ below ground surface. The casing was pulled back with the Bucyrus Erie 2W cable-tool drill rig that drilled the borehole.

The estimated volume of water added to the borehole during drilling is $160 \mathrm{gal}$; the estimated volume of water removed during the two-phase development process is 1068 gal. Water added to the borehole was obtained from the Richland municipal water supply in the 3000 Area and was stored onsite in 55-gal steel drums. Field monitoring for contamination was performed by PNL geologists and radiation protection technologists from Westinghouse Hanford Company. No contamination was detected, so the vadose zone was not sampled for hazardous constituents.

Well development consisted of bailing the completed well with the cabletool rig and dart bailer until the water was essentially sediment free; continued development was performed with a 1.5-hp submersible pump. The well was considered developed when water samples were consistently below the 5-NTU

(a) Volclay is a registered trademark of American Colloid Company, Arl ington Heights, Illinois. 
criteria (EPA 1986); water samples were analyzed with a Hach Portable Turbidimeter. Results from rapid water analysis sent to UST on October 20, 1988, indicated that the development water could be discharged to the ground; however, because of the proximity of the 1166 Building parking area, the water was contained. $\mathrm{N}$ aquifer test was performed. The HydroStar pump was installed on November 4, 1988; this completed construction activities at well 699-S43-E12.

\section{WELL 5 (699-S37-E14)}

Drilling at well 699-S37-E14 began October 3, 1988. The well was drilled to a depth of $62.5 \mathrm{ft}$ below ground surface by October 10, 1988. The sediments encountered were primarily silty sandy gravels. The middle Ringold member was encountered at approximately $50 \mathrm{ft}$ below ground surface; static water level during well completion was $51.17 \mathrm{ft}$ below ground surface.

Well 699-S37-E14 was completed at a depth of $63.0 \mathrm{ft}$ below ground surface. Final well materials include $15.75 \mathrm{ft}$ of 20-slot Johnson Division type 304 stainless steel screen (4 in. dia) and $50 \mathrm{ft}$ of 4 -in. Johnson Division type 304 stainless steel casing. Factory-welded stainless steel casing centralizers were located at 27.25 and $57.75 \mathrm{ft}$ below ground surface. The annular seal consists of a 16-30 mesh Colorado silica sand pack along the screened interval to $3.35 \mathrm{ft}$ above the top of the screen, $5.73 \mathrm{ft}$ of Volclay bentonite pellets, $19.79 \mathrm{ft}$ of 8-20 mesh granular bentonite crumbles, $15.18 \mathrm{ft}$ of Portland cement grout, and an air-entrained concrete seal that reaches $3.2 \mathrm{ft}$ below ground surface. The casing was pulled back with the Bucyrus Erie 22W cable-tool drill rig that drilled the borehole.

The estimated volume of water added to the borehole during drilling is $150 \mathrm{gal}$; the estimated volume of water removed during the two-phase development process is $788 \mathrm{gal}$. Water added to the borehole was obtained from the Richland municipal water supply in the 3000 Area and was stored onsite in 55-gal steel drums. Field monitoring for contamination was performed by PNL geologists and radiation protection technologists. No contamination was detected, so the vadose zone was not sampled for hazardous constituents. 
We11 development consisted of bailing the completed well with the cabletool rig and dart bailer until the water was essentially sediment free; continued development was performed with a 1.5 -hp submersible pump. The well was considered developed when water samples were consistently below the 5-NTU criteria (EPA 1986); water samples were analyzed with a Hach Portable Turbidimeter. Devel opment water was discharged to the ground after receiving acceptable results from a rapid water analysis sent to UST on October 12, 1988. N aquifer test was performed. The HydroStar 'pump was instal led on November 3, 1988; this completed construction activities at we11 699-S37-E14.

\section{RESULTS OF GROUND-WATER SAMPLE ANALYSIS}

Results of the analysis of ground-water samples from the five new wells are presented in Appendix H. The samples were collected on November 7, 1988. The reported results were determined by UST under an analytical support contract to PNL. Results for all constituents were below those allowable under drinking water standards. A single volatile organic constituent (methylene chloride) was detected in three of the samples. Methylene chloride is not expected to be present in the ground water at the site, and its presence in the samples may have indicated that the samples were contaminated with methylene chloride. The wells were resampled on November 11 and 14 and analyzed by PNL. Methylene chloride was below detection in these samples, indicating samples collected November 7 were contaminated with this constituent during the sampling process.

Ground-water samples collected on November 7, 1988, from each well were also analyzed by PNL using a gas chromatography/electron capture detection/ flame ionization detector with attached purge and trap on November 9, 1988. Trace concentrations of several volatile organic constituents were observed during this analysis, although all concentrations were well below the maximum contaminant level identified by the U.S. Environmental Protection Agency. 


\section{REFERENCES}

Bryce, R. W. 1989. Well Installation and Ground-Water Samplinq Plan for the 1100 Area Environmental Monitorins Wells. PNL-6815, Pacific Northwest Laboratory, Richland, Washington.

EPA. 1986. Resource Conservation and Recoverv Act (RCRA) Ground-Water Monitoring Technical Enforcement Guidance Document. OSWER 99S0.1,

U.S. Environmental Protection Agency, Washington, D.C. 
APPENDIX A

GEOPHYSICAL AND GEOLOGIC LOGS FOR WELL 1 (699-S41-E13A) 


\section{APPENDIX A \\ GEOPHYSICAL AND GEOLOGIC LOGS FOR WEL 1 (699-S41-E13A)}

This appendix contains the Well Completion/Inspection Report, as-built diagram, notes from the sampling pump installation, the natural gamma log, and the geologists' logs for well 1 (699-S41-E13A) in the 1100 Area. 


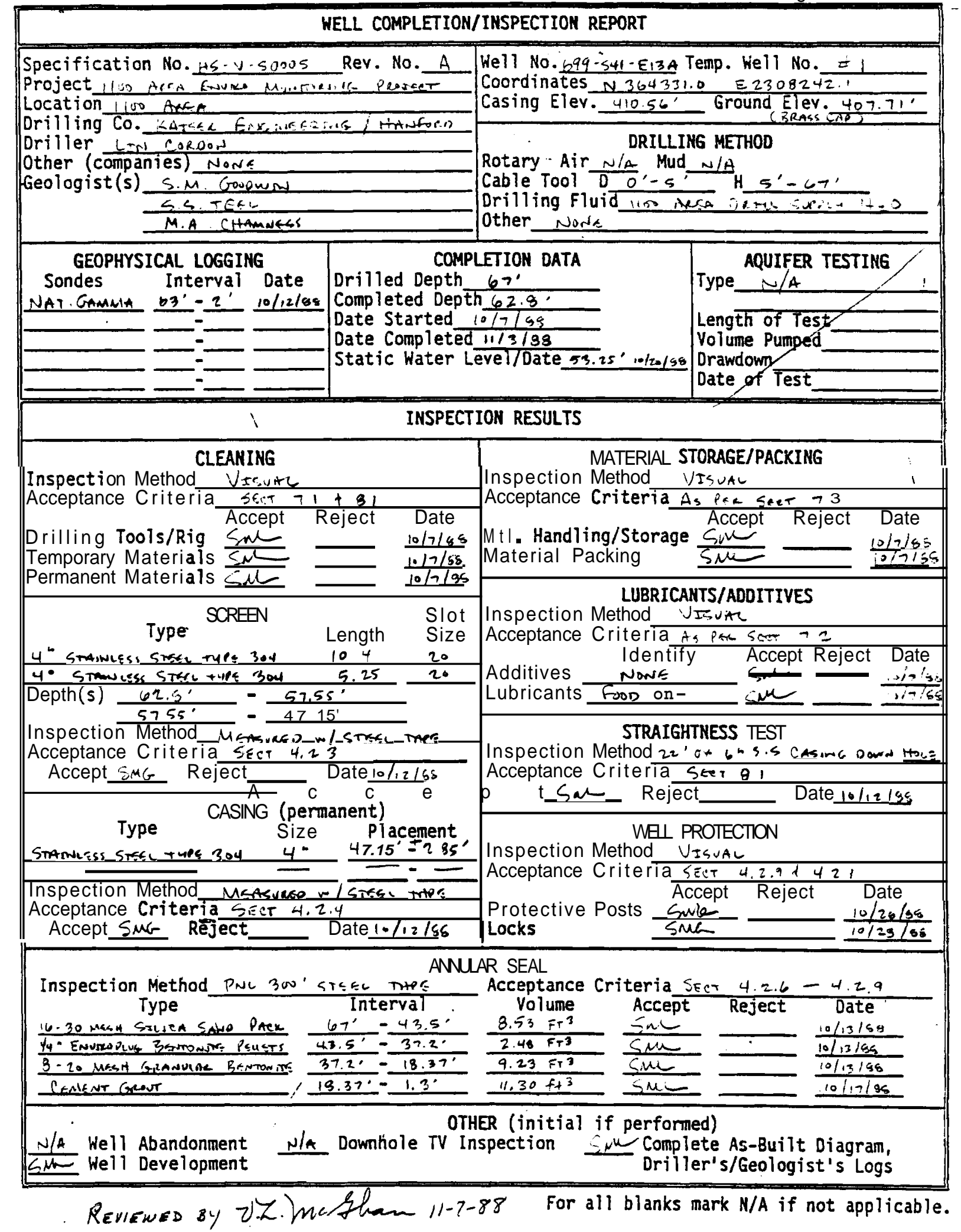




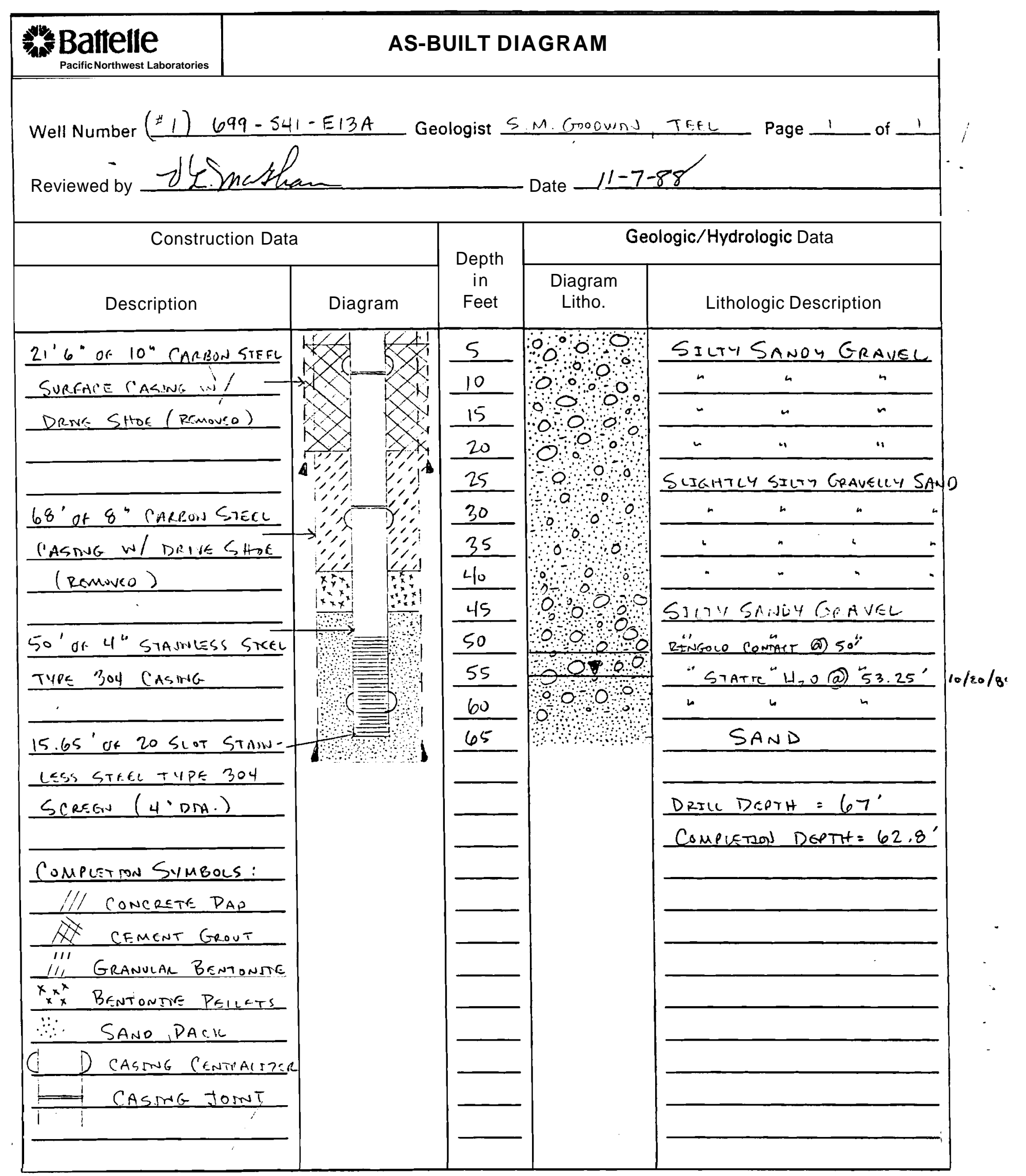


SAMPLING PUMP INSTALLATION IN

GROUND-WATER MONITORING WELLS

Site: 1100 AfEA

Monitoring Well Number: $(\# 1) 699-541-E_{13} A$

Depth to Water: $54.7^{\circ}$

Depth to Bottom: $62.87^{\circ}$ Reported Depth to Bottom: $62.8^{\circ}$

Pump Type: Positive Displacement Piston

Pump Model : Hydrostar HS-8001

Date Instal led: $11 / 3 / 98$

Installed By: KEH: OLNW Amos. LOUTS WATKSWS, LENNH COROON

Pump Discharge Pipe Description:

$$
\begin{aligned}
& 610^{\circ} \text { SEctions of } 3 / 4^{\prime \prime} \text { STAMULSSS STEEL DISCHAtece PAPE }
\end{aligned}
$$

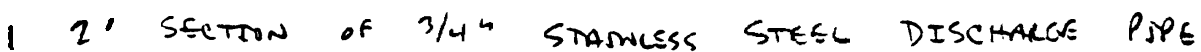

$$
\begin{aligned}
& 2^{\prime} \text { SECTow } 15 \text { BETwEEN } 10^{\prime} \mathrm{d} 12^{\prime} \text { (From PJuP base plats) }
\end{aligned}
$$

Additional Comments:

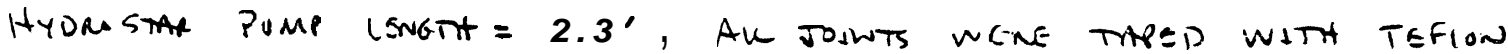
DRJLLSR WORE COTtON GLOVES DURWG mRSTAUATION

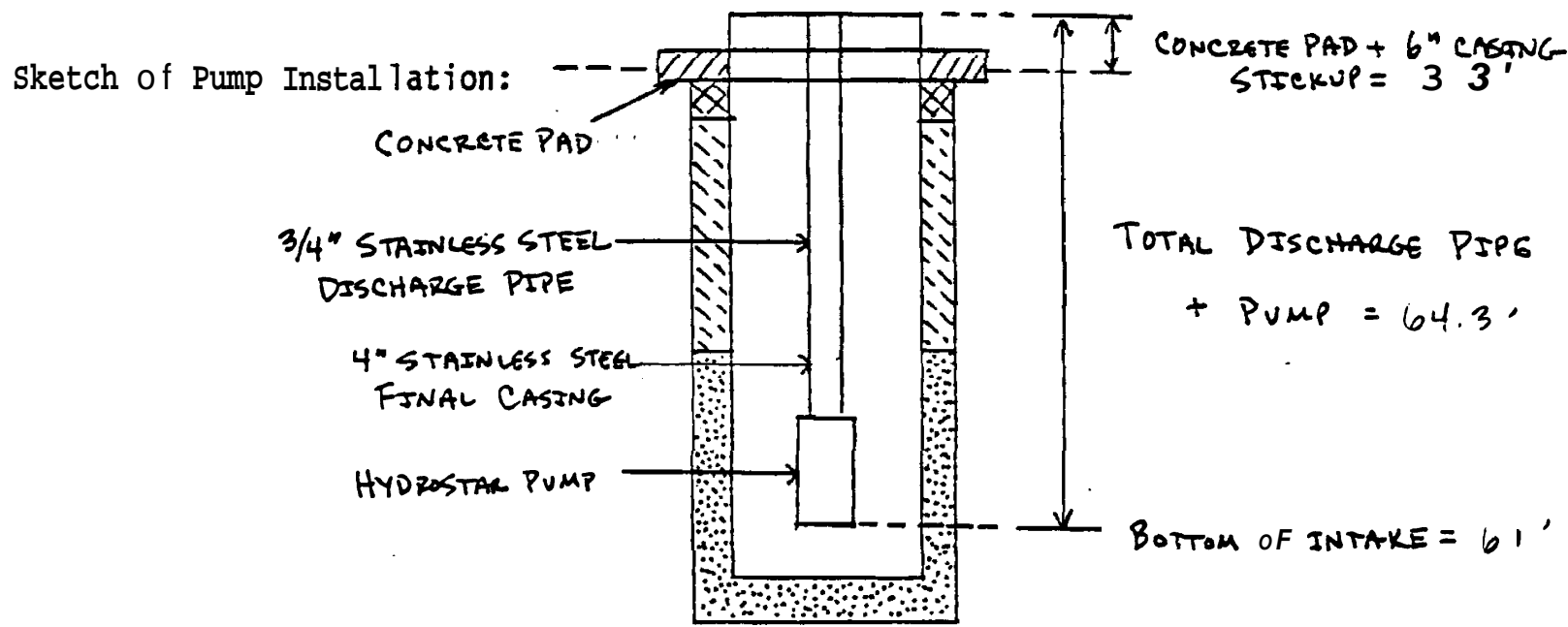

Completed By: Shankertool.

Date: $11 / 3 / 39$ 


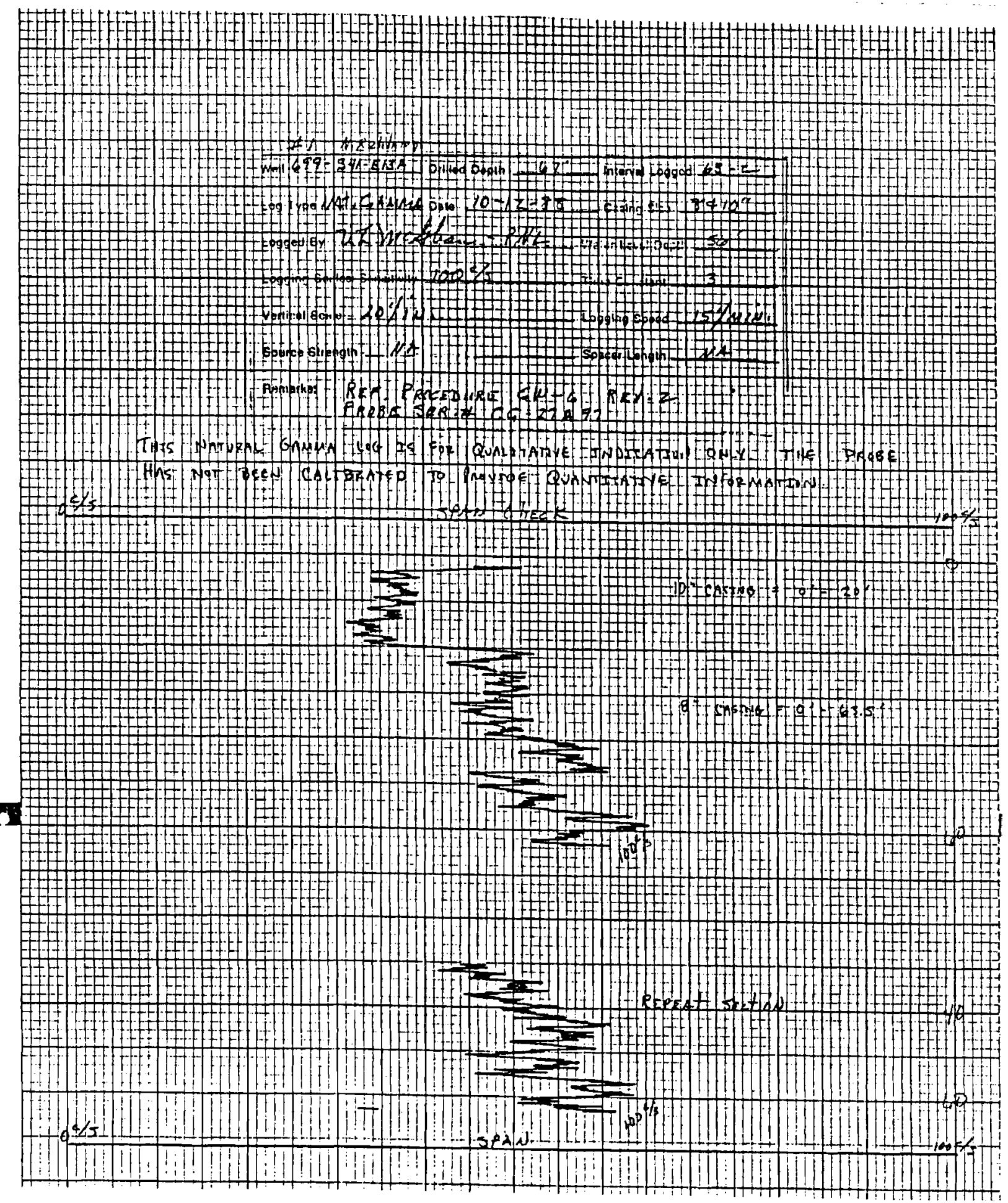

\section{A. 6}


1 or 2

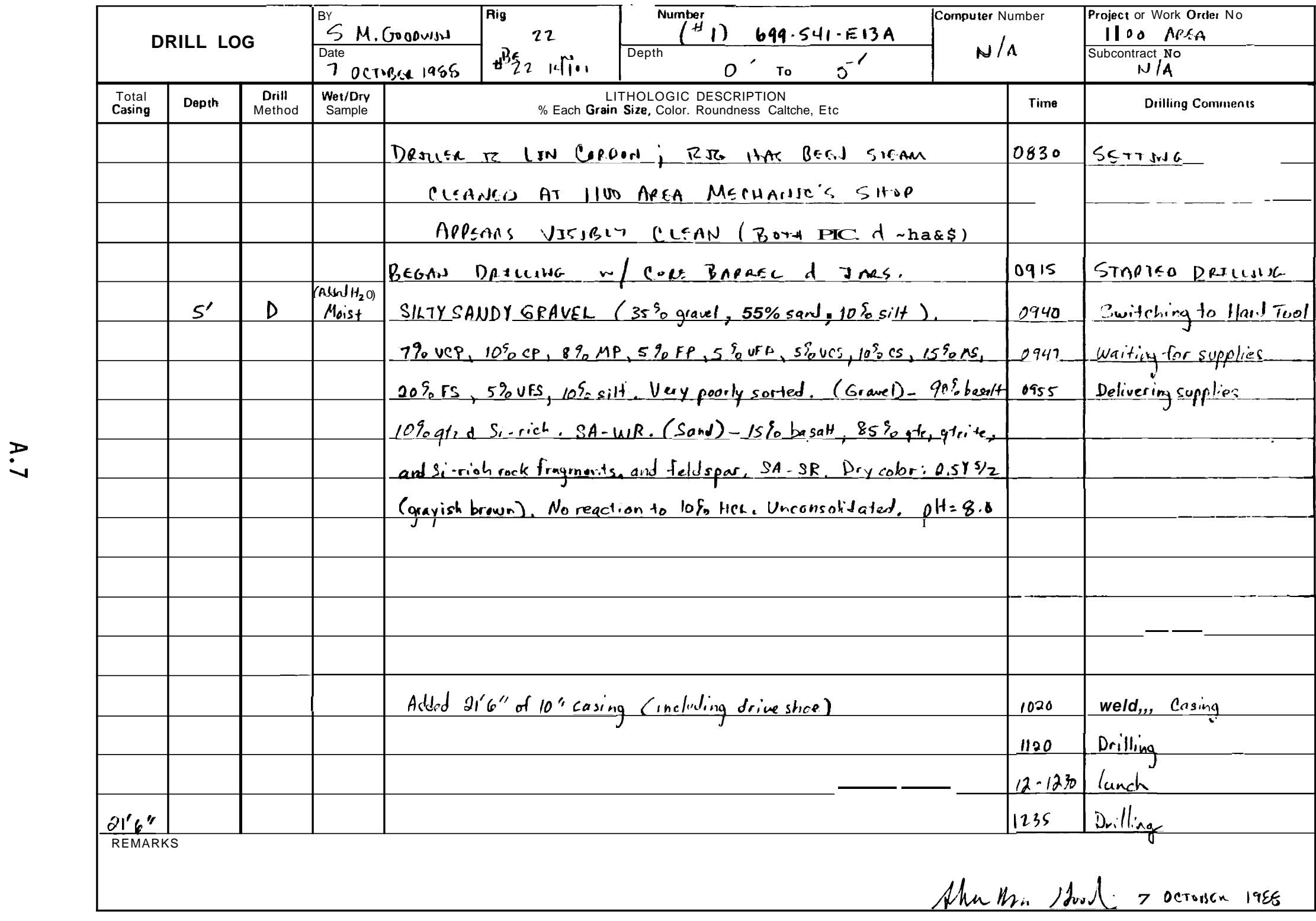

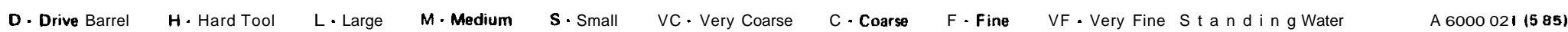


(S8.9) 120.00098

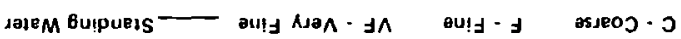

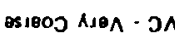

Hews - $s$ un!paw - $W$

$26897 \cdot 7$

POOL PABH - H

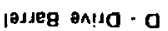

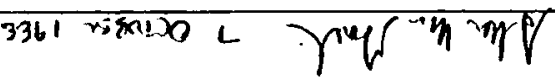

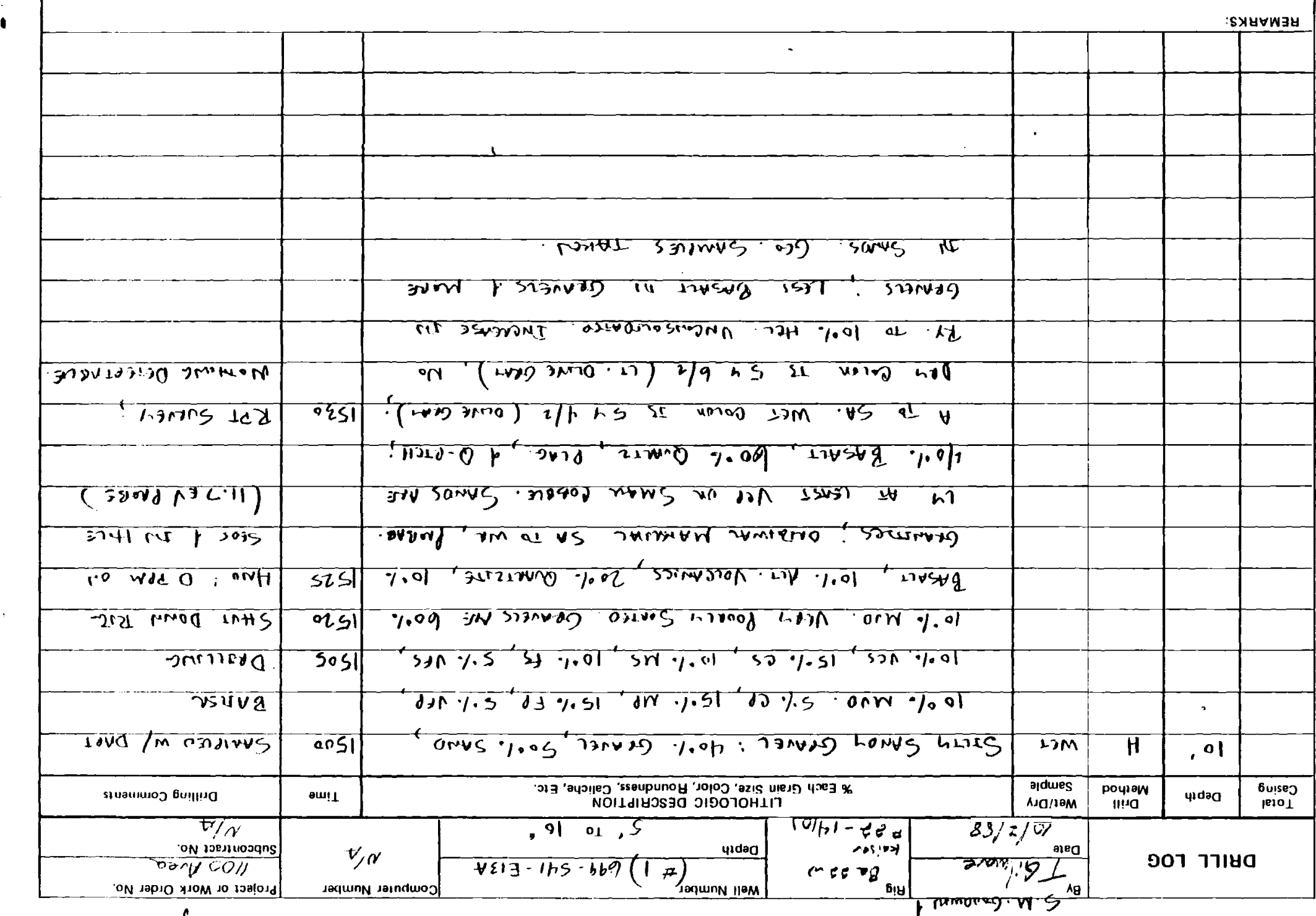

$2 \% \rho$ 


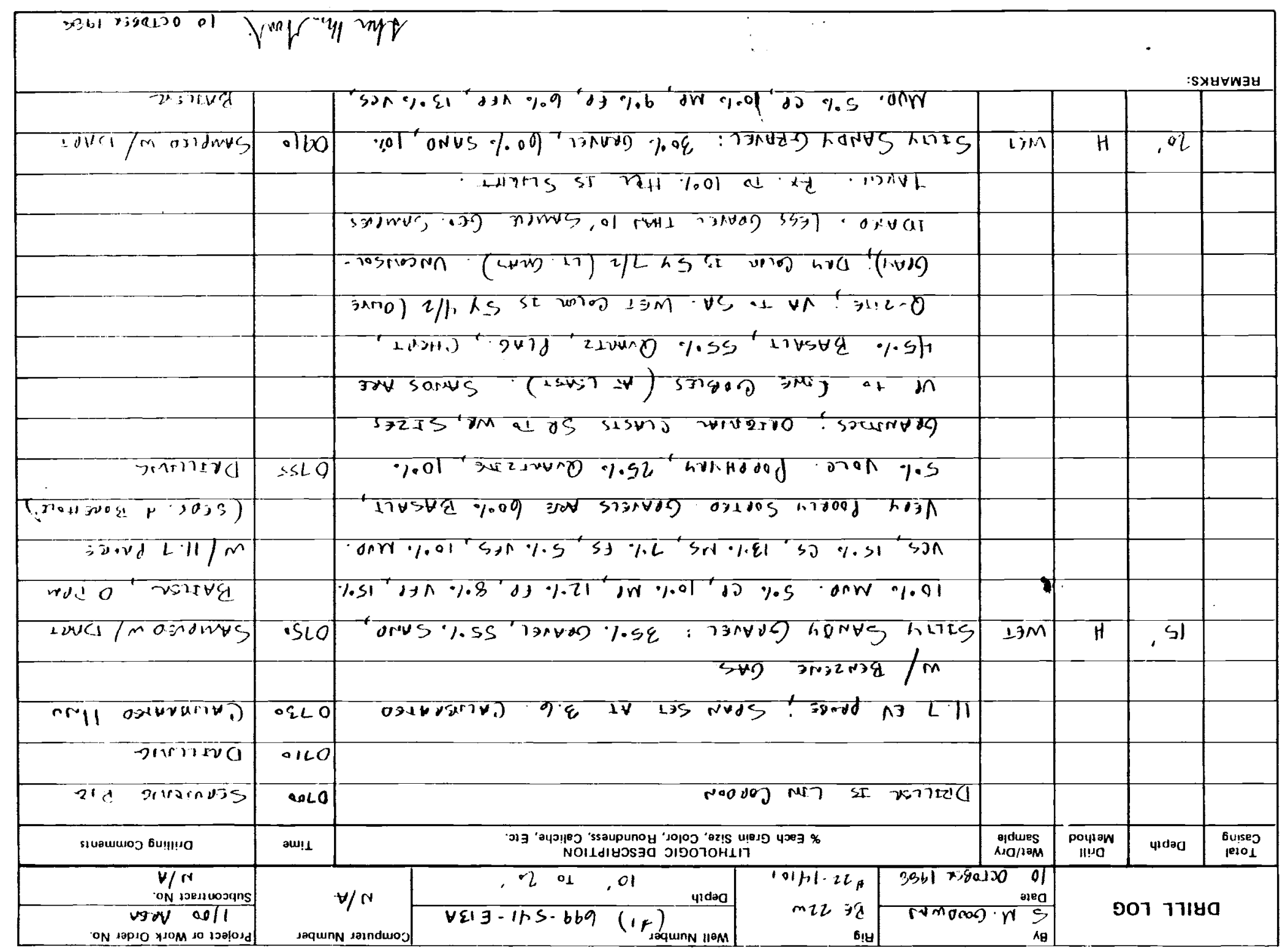


(s8 9) เzo $0009 \forall$

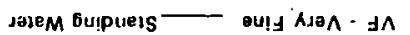

au!y - y

as

asheoj Rian - כ八

Ilews - $\mathbf{s}$

แกрมา - w

$26807-7$

1001 P.eH - H

0

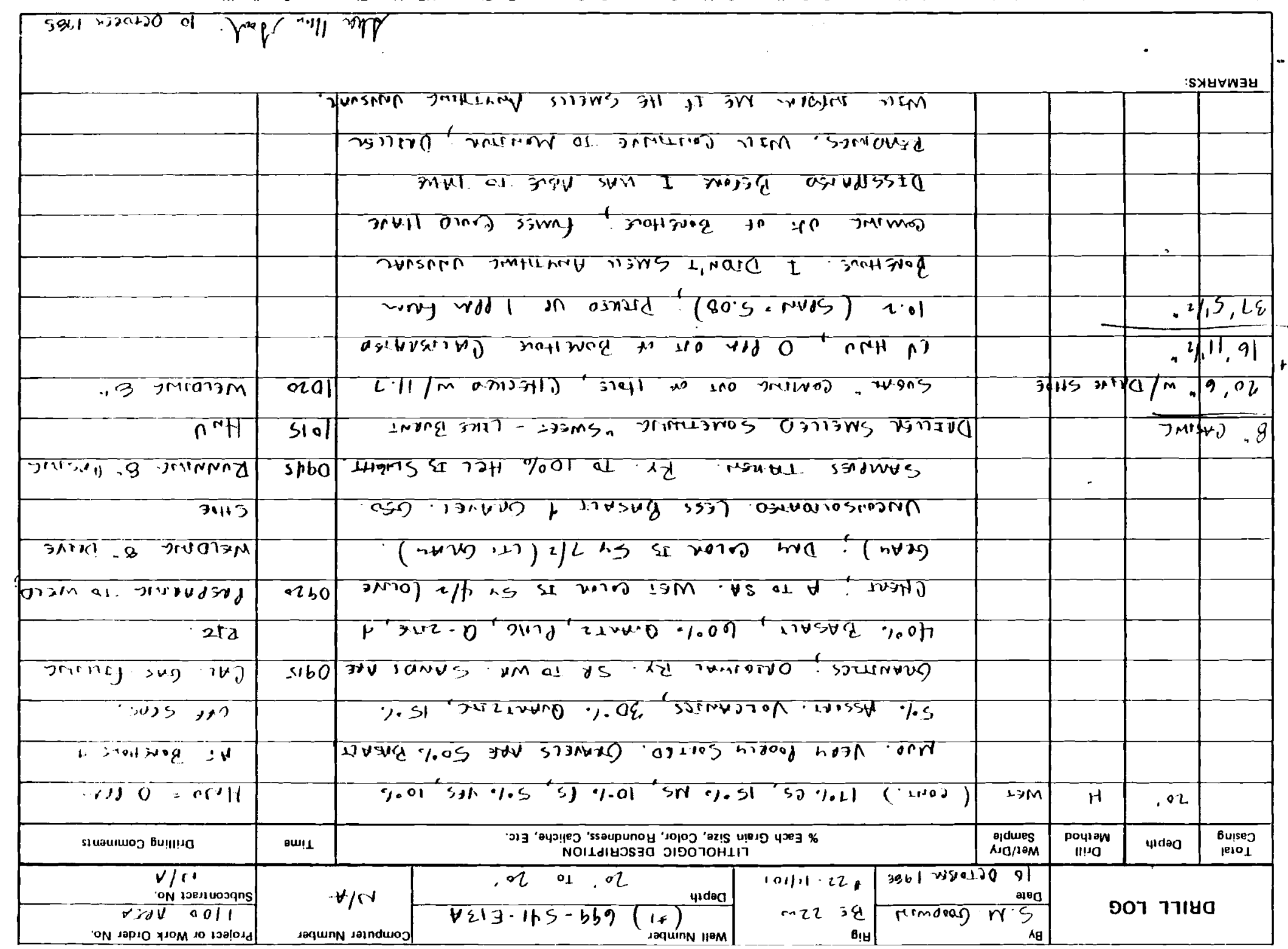

5,02 
0,6

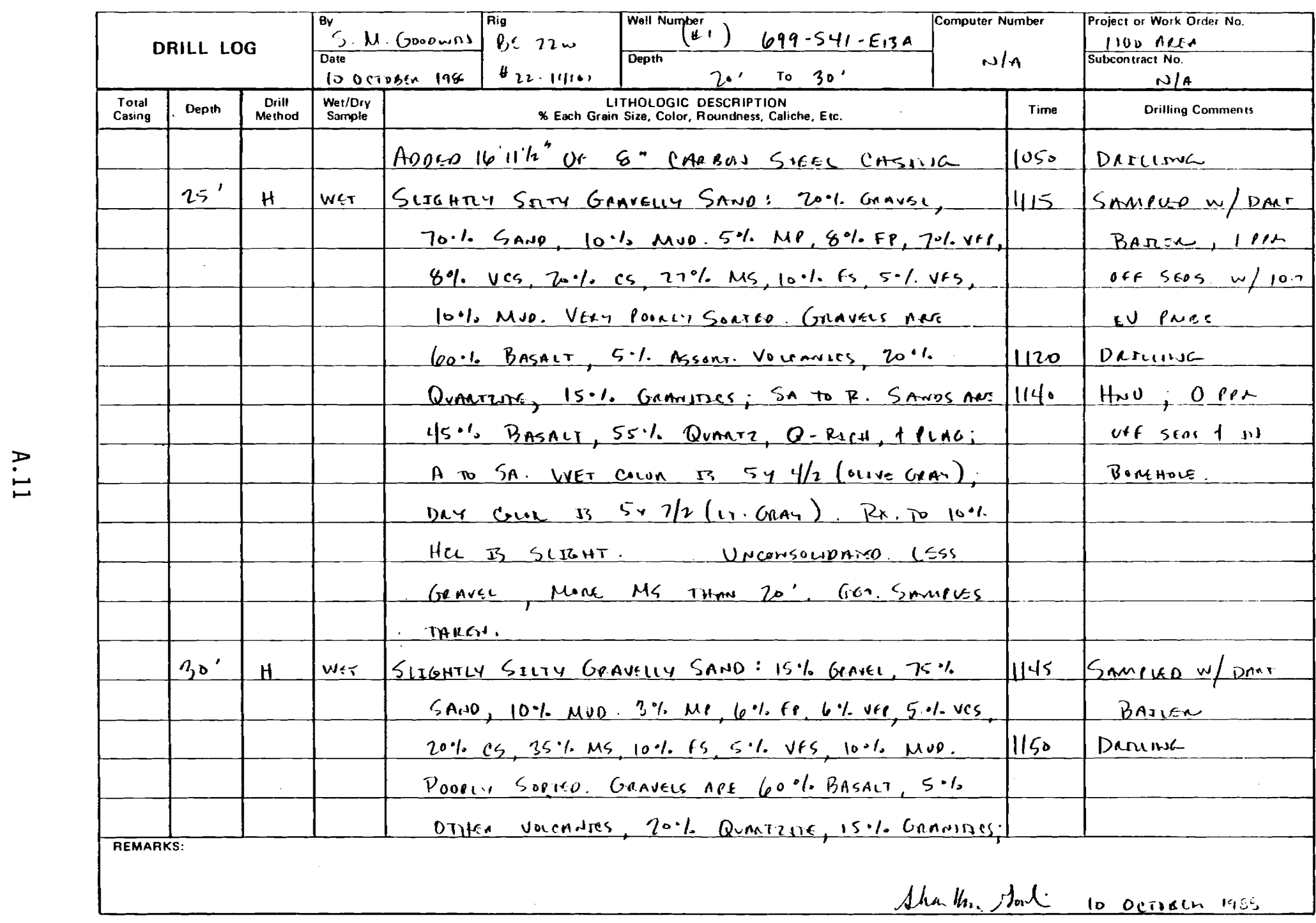

D. Drive Barrel H. Hard Tool L. Large M-Medium S.Small VC.Very Coarse C.Coarse F. Fine VF. Very Fine - Standing Water A6000 021 (5.85) 
4 of 5

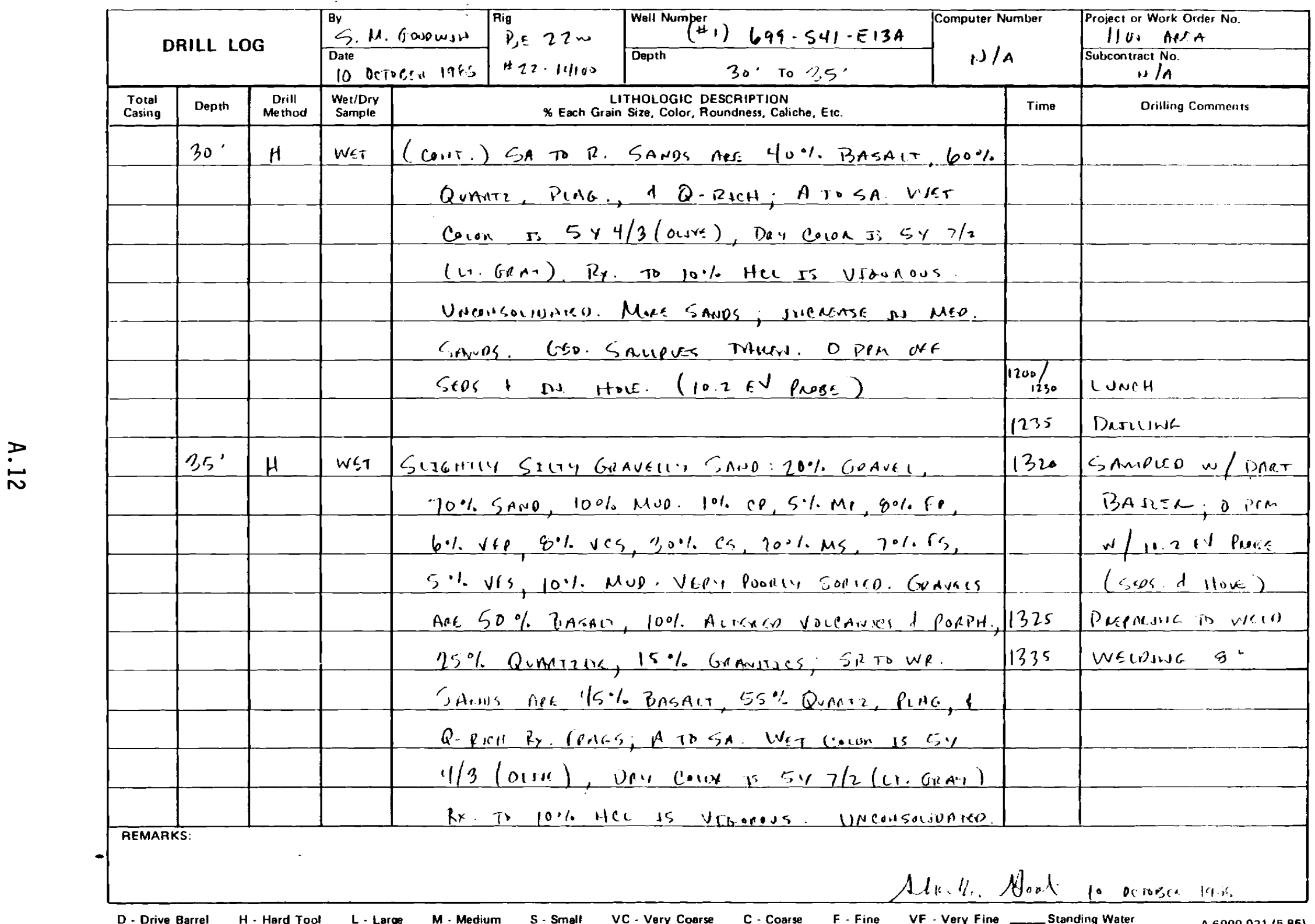




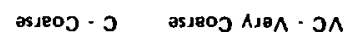

flews - S untpaw - W

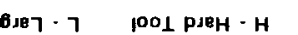

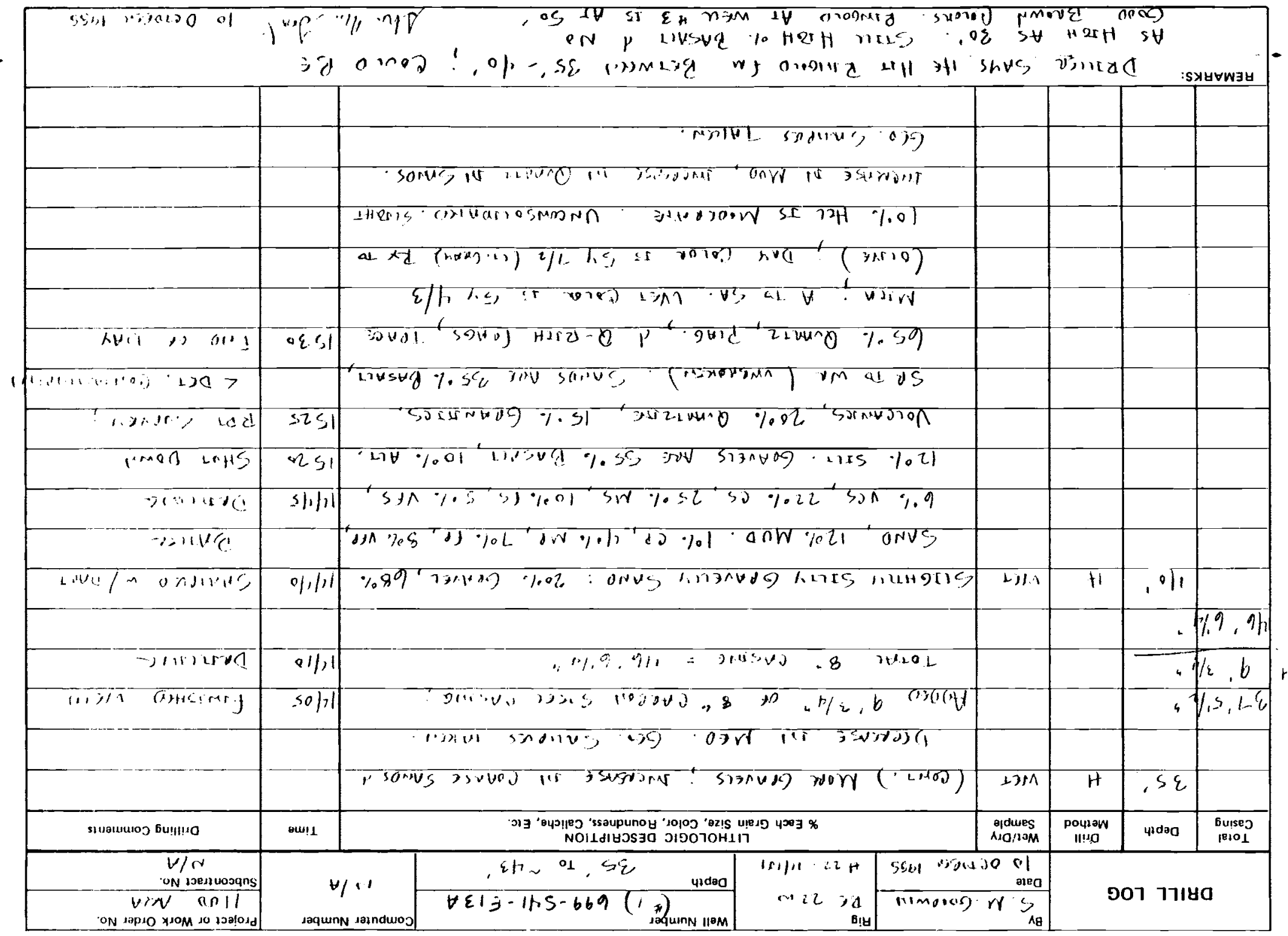




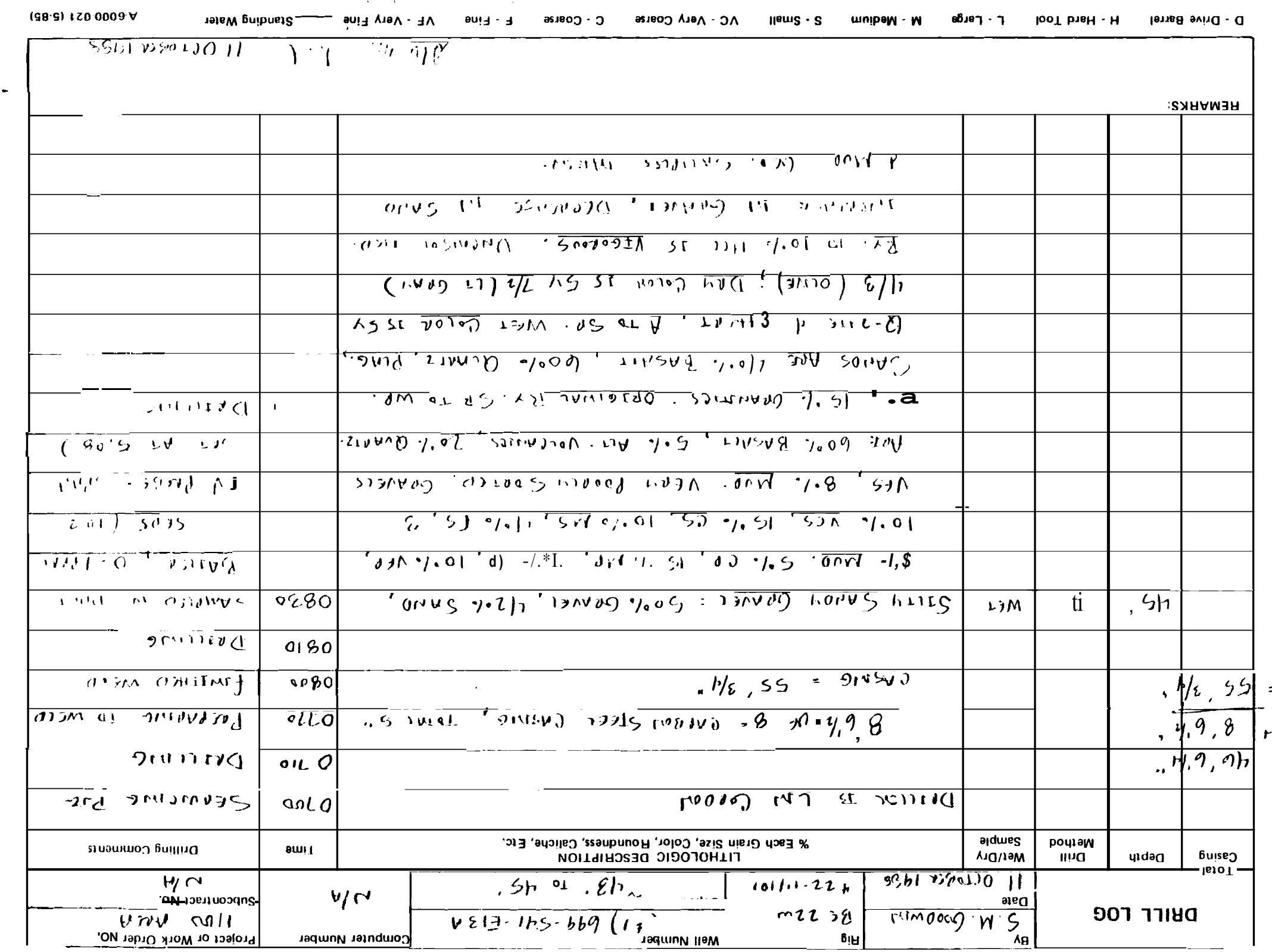


2. of 4

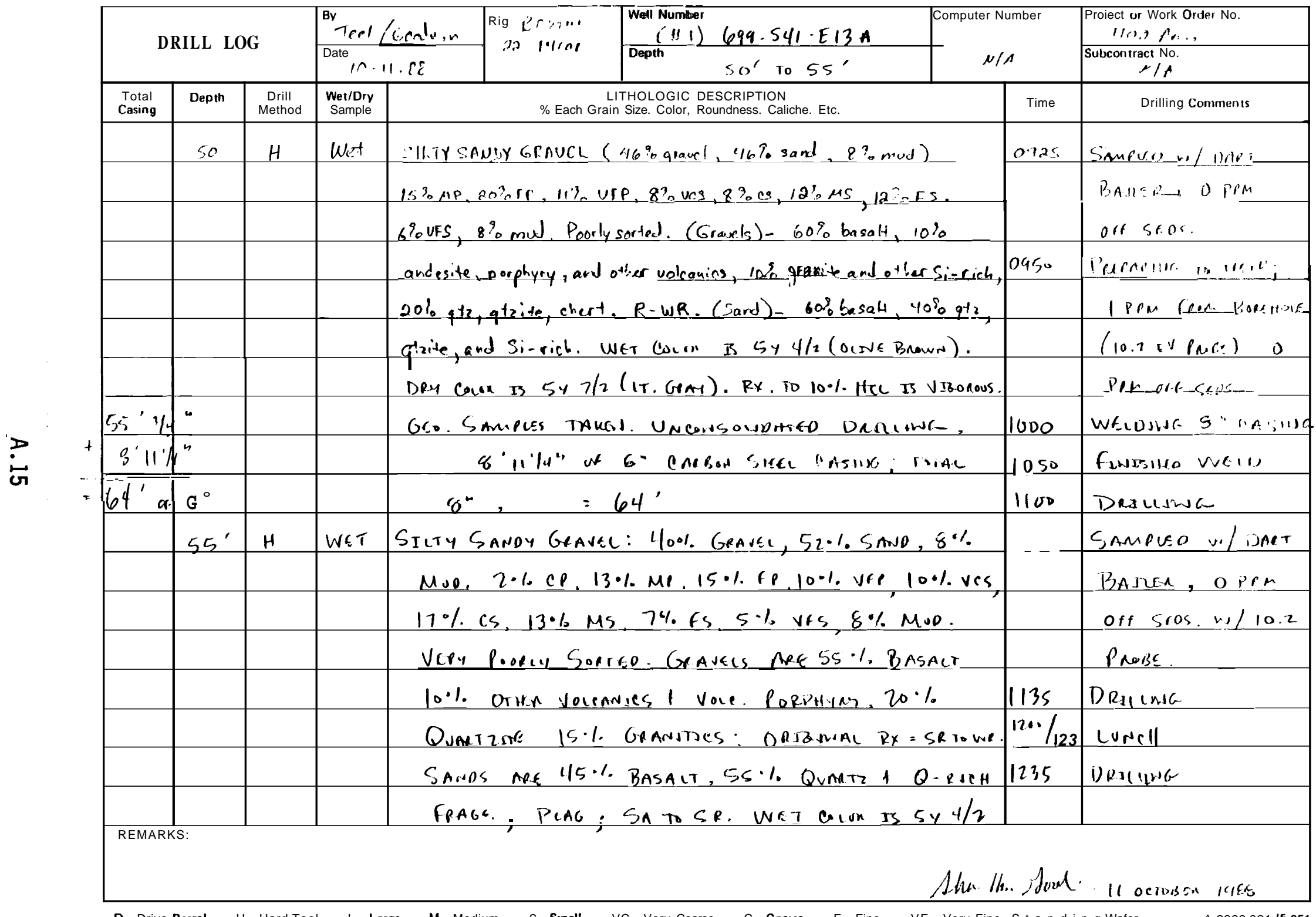




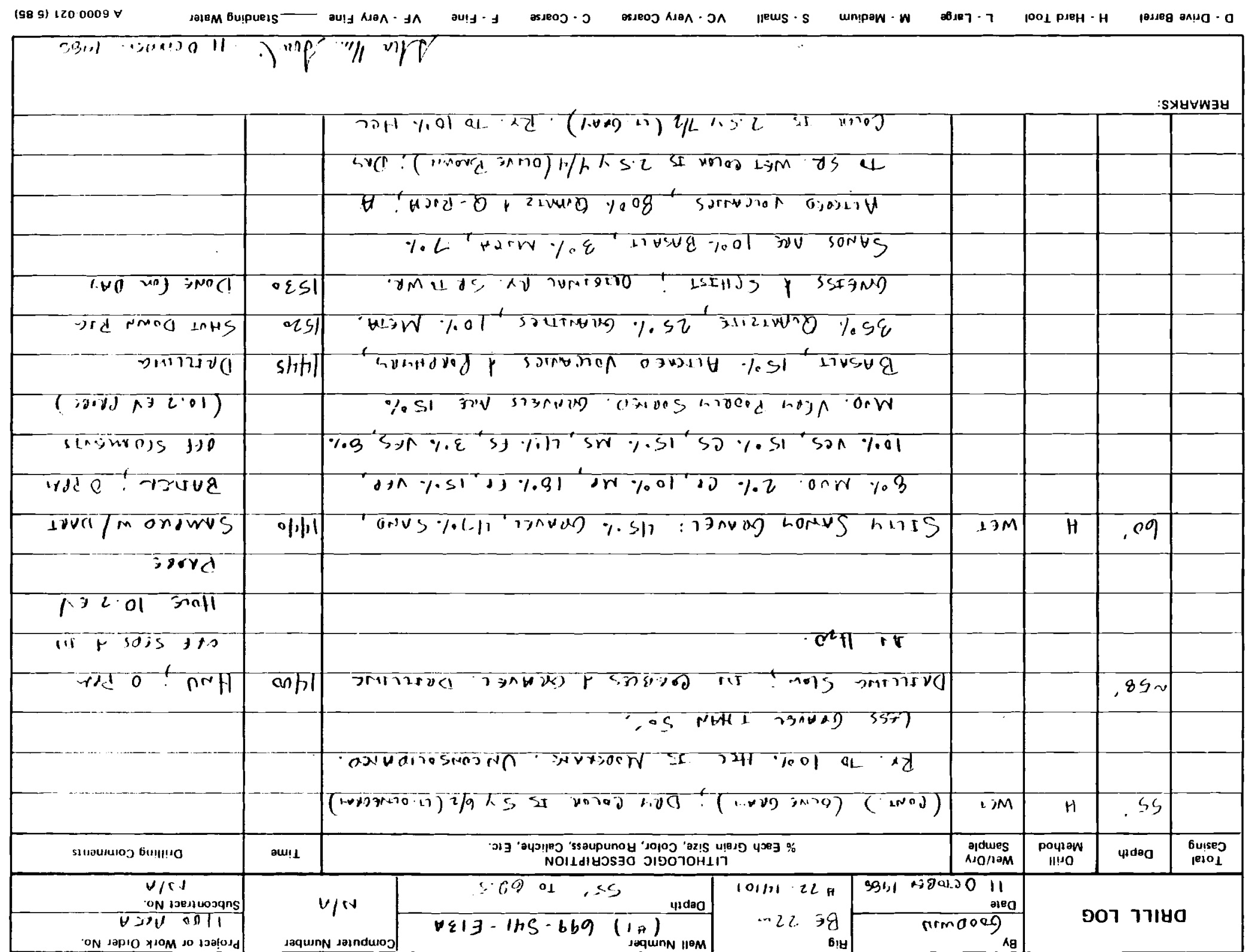


11 or 11

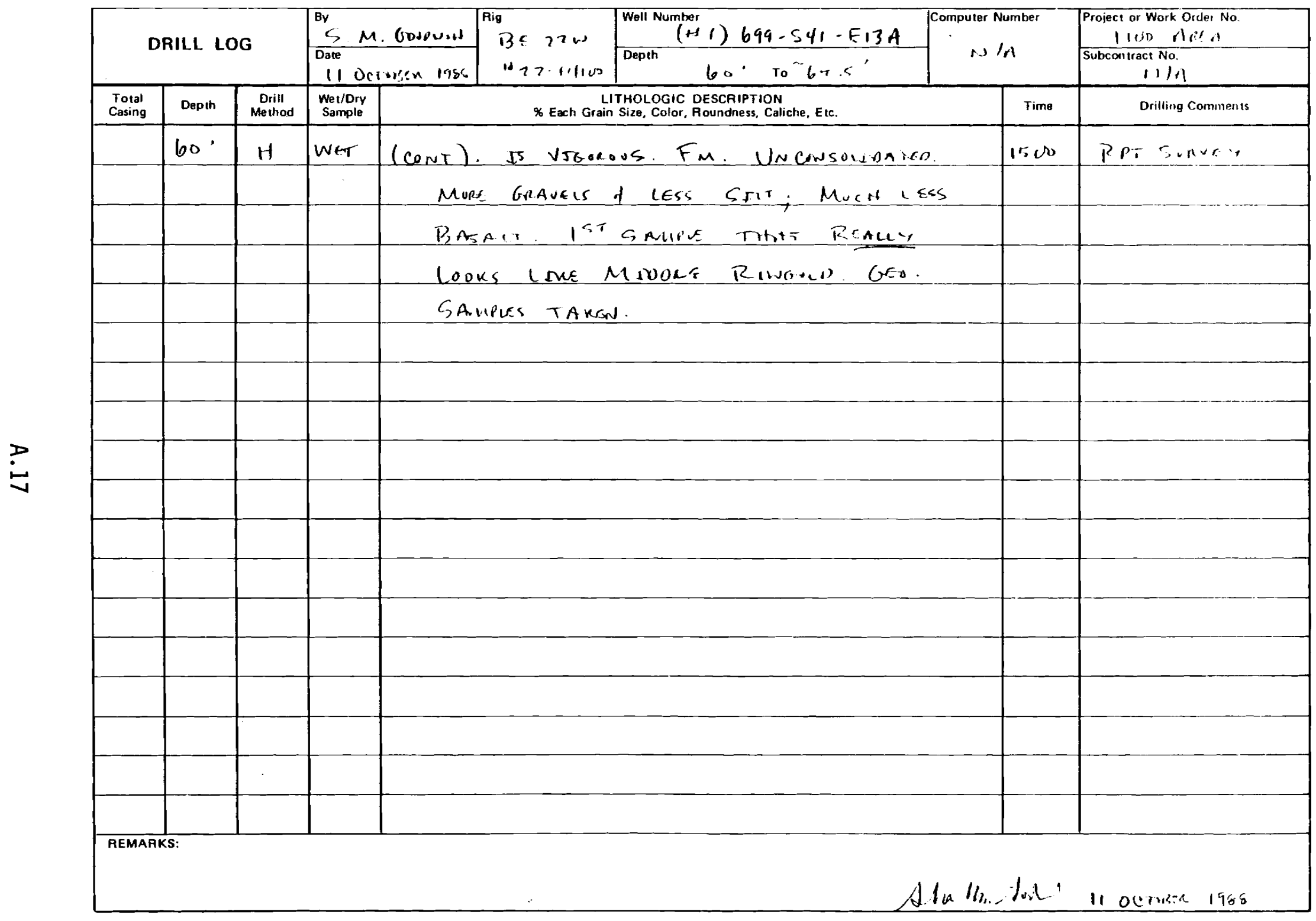

D. Drive Barrel H-Hard Tool L-Large M-Medium S-Small VC-Very Coarse C - Coarse F-Fine VF-Very Fine _-Standing Water 


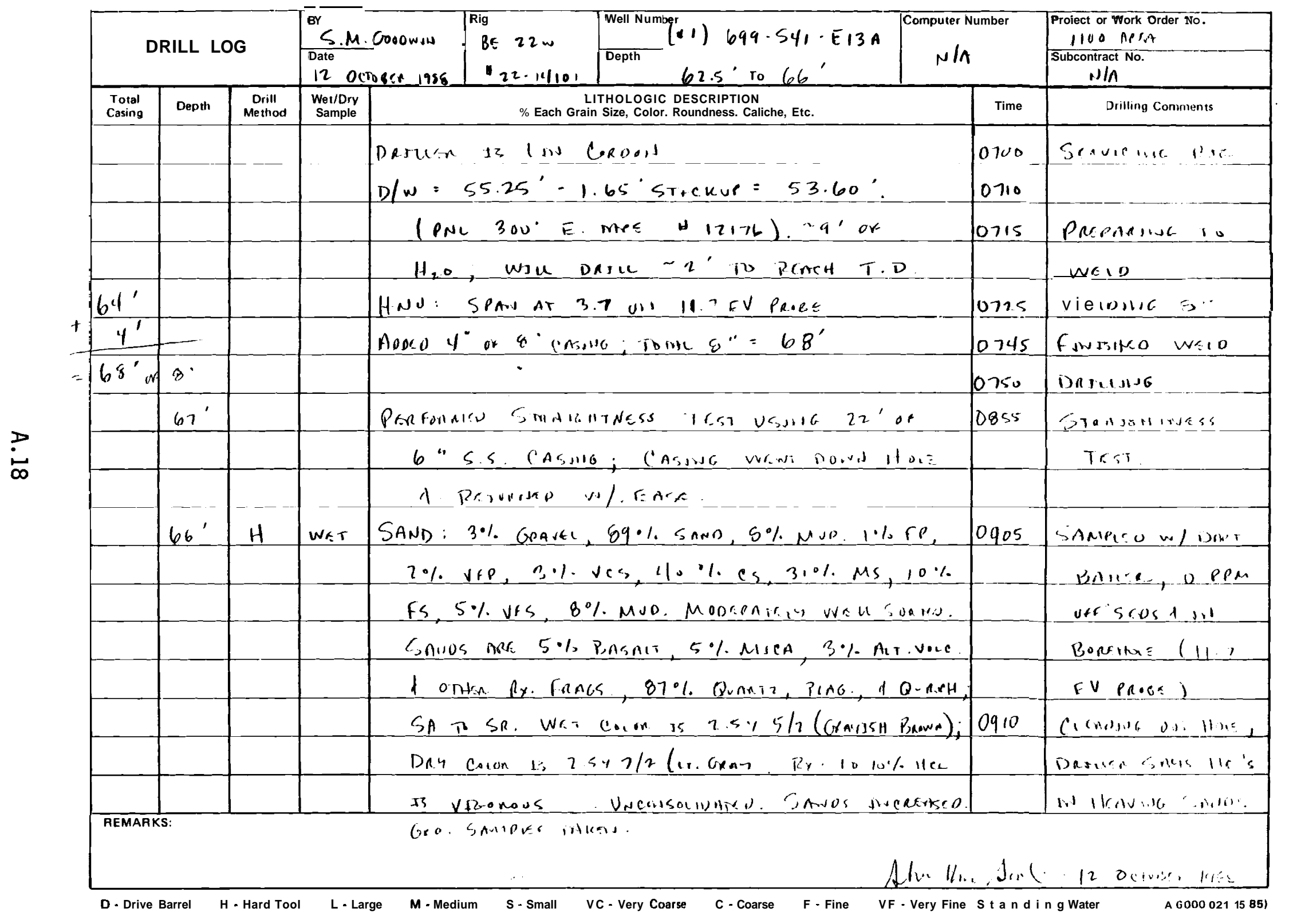


of 4

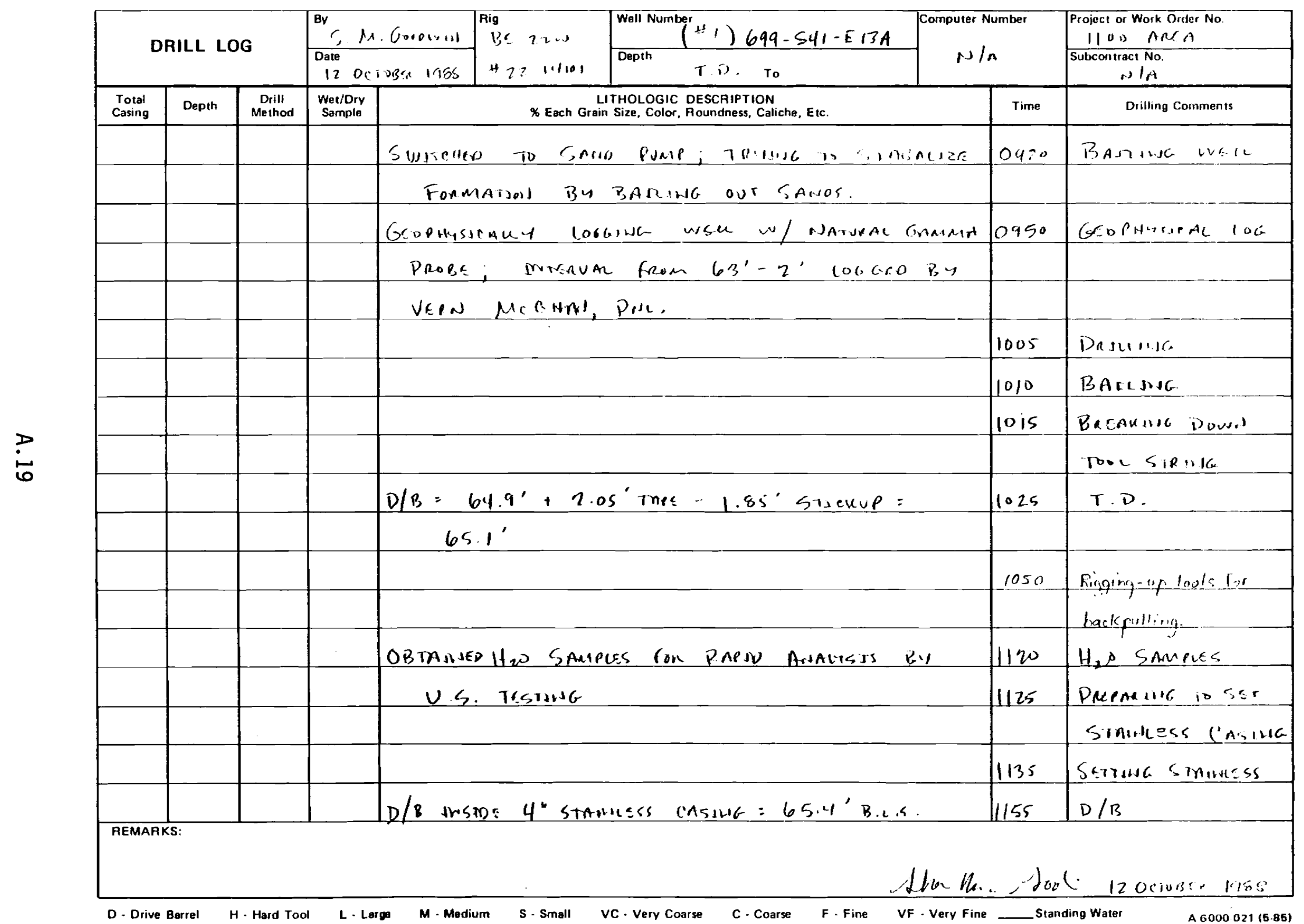




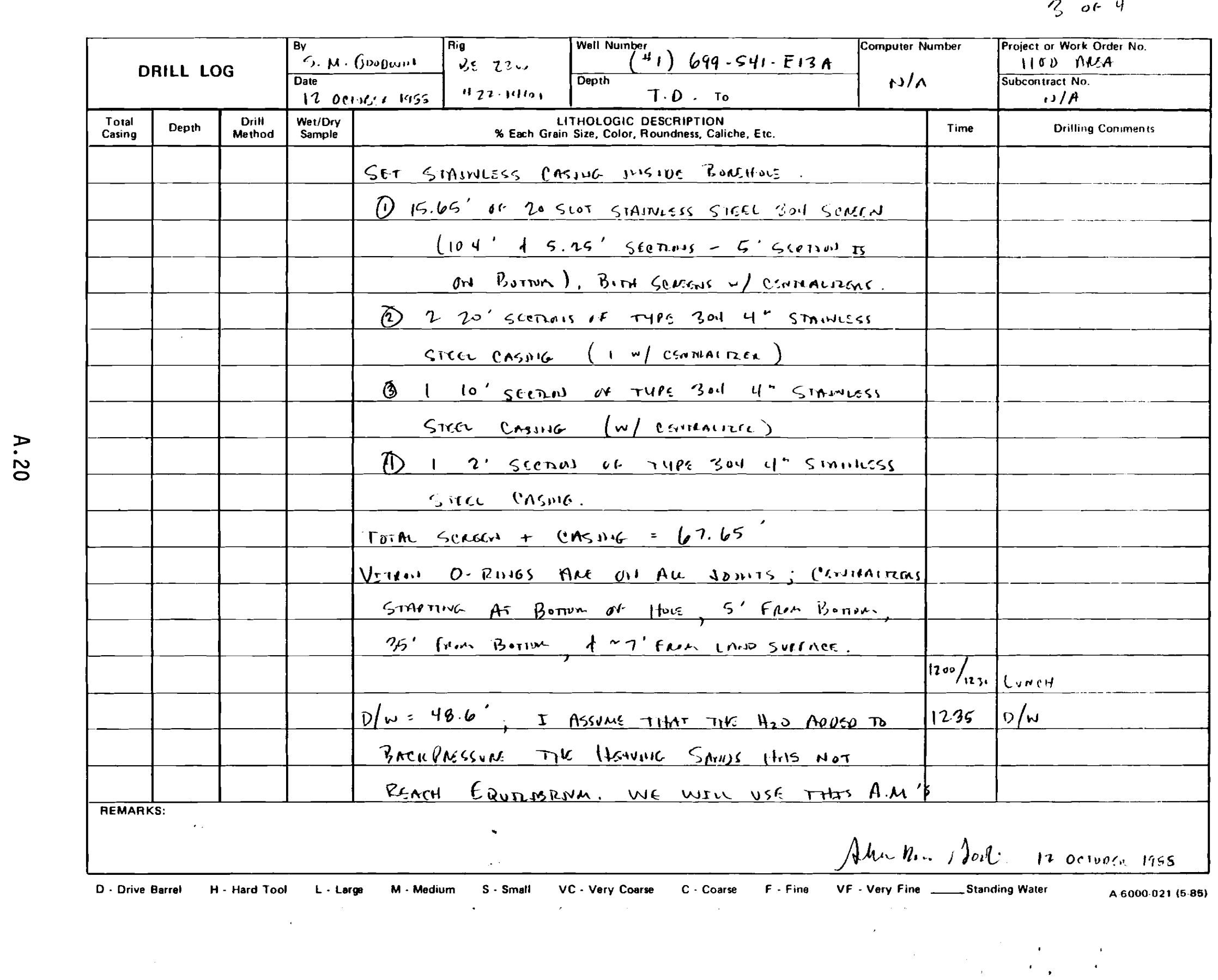


$10+4$

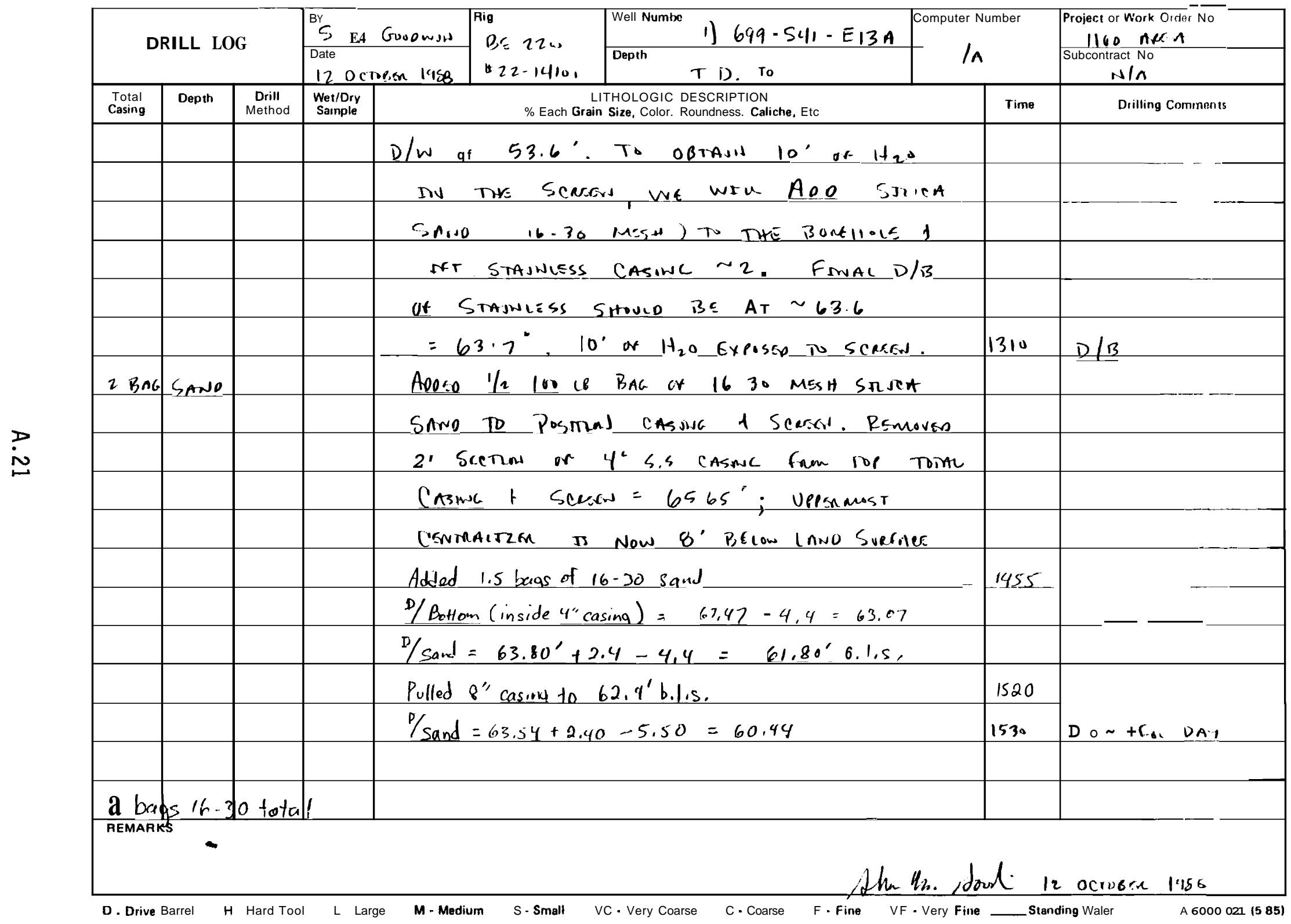




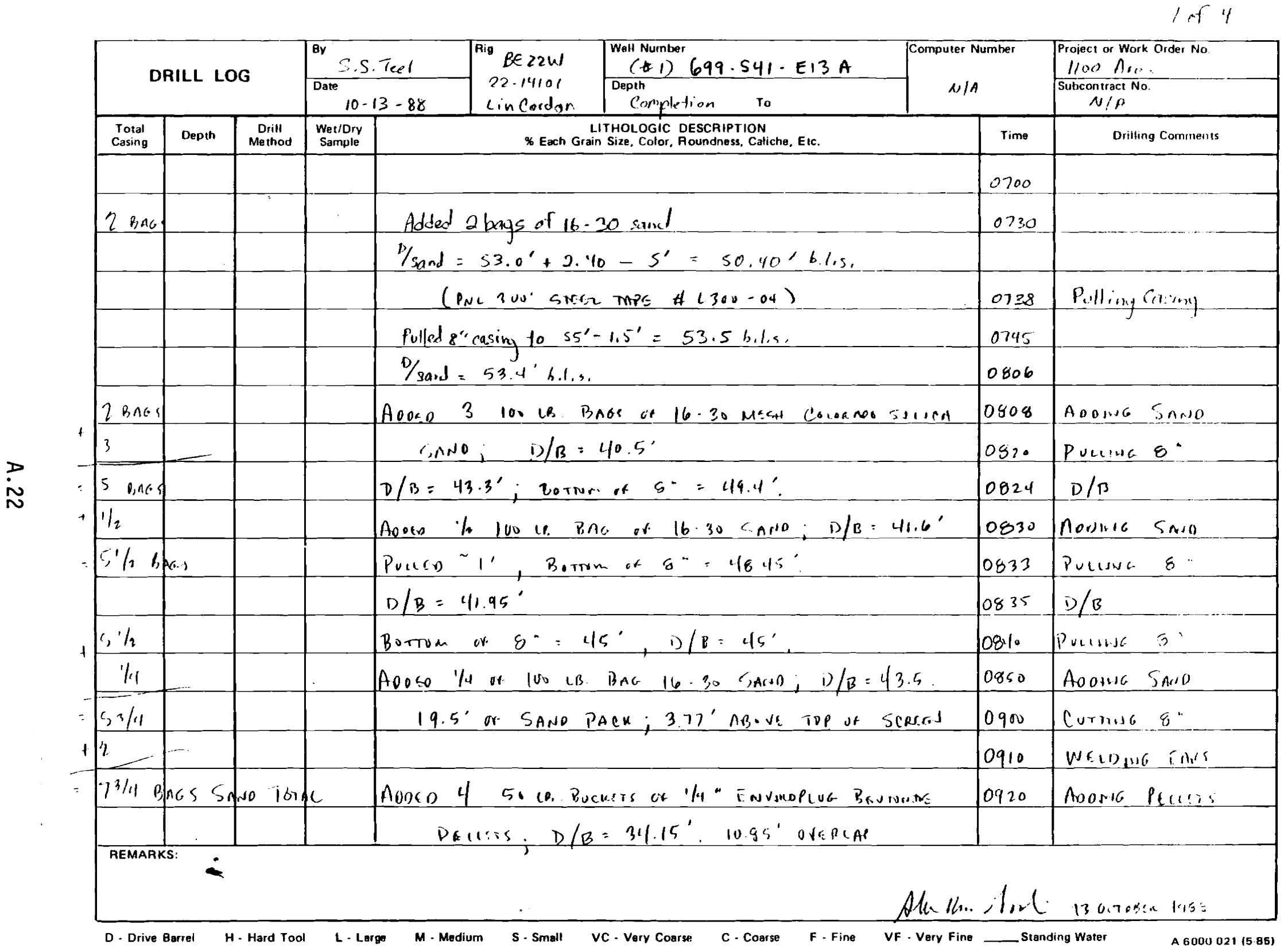




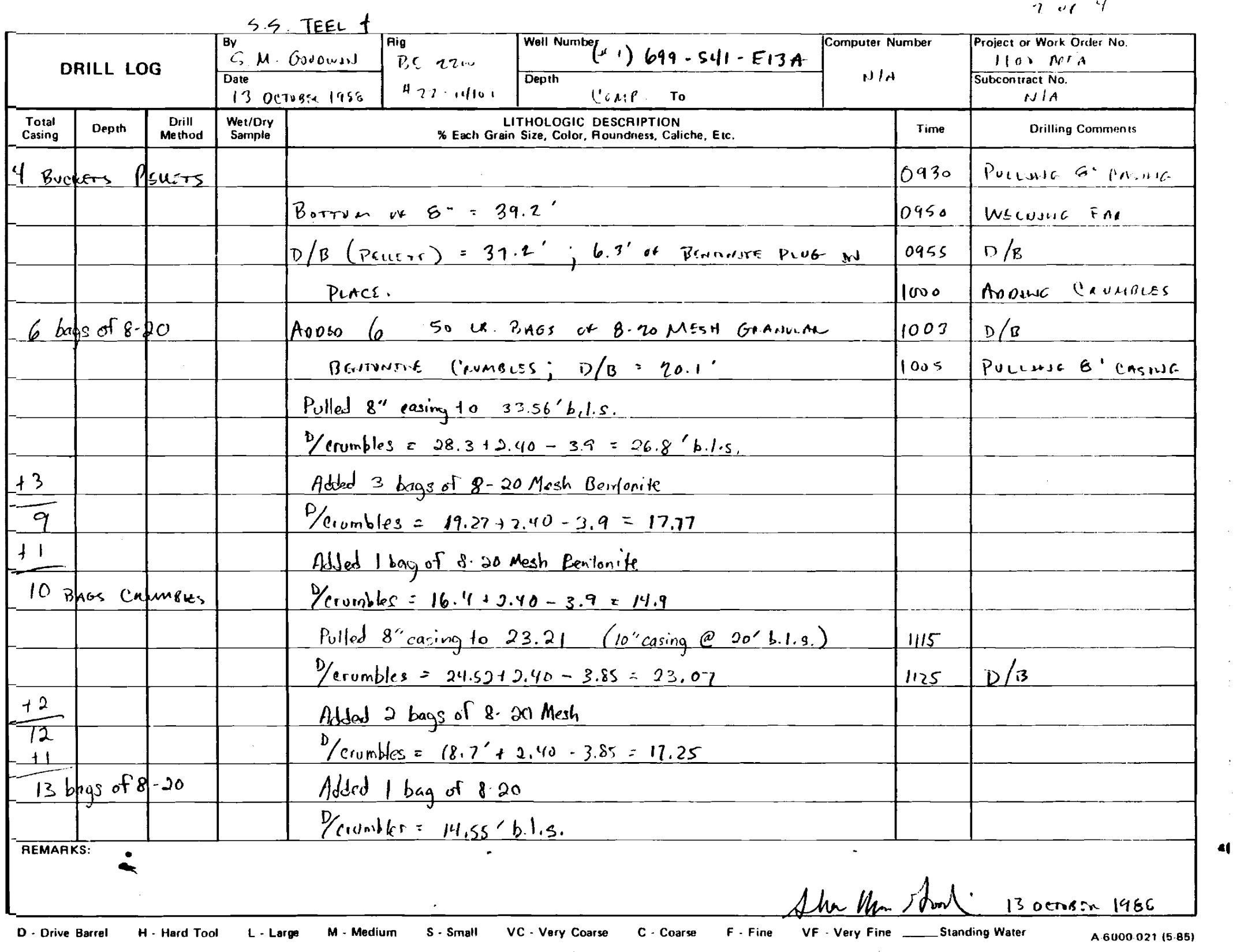




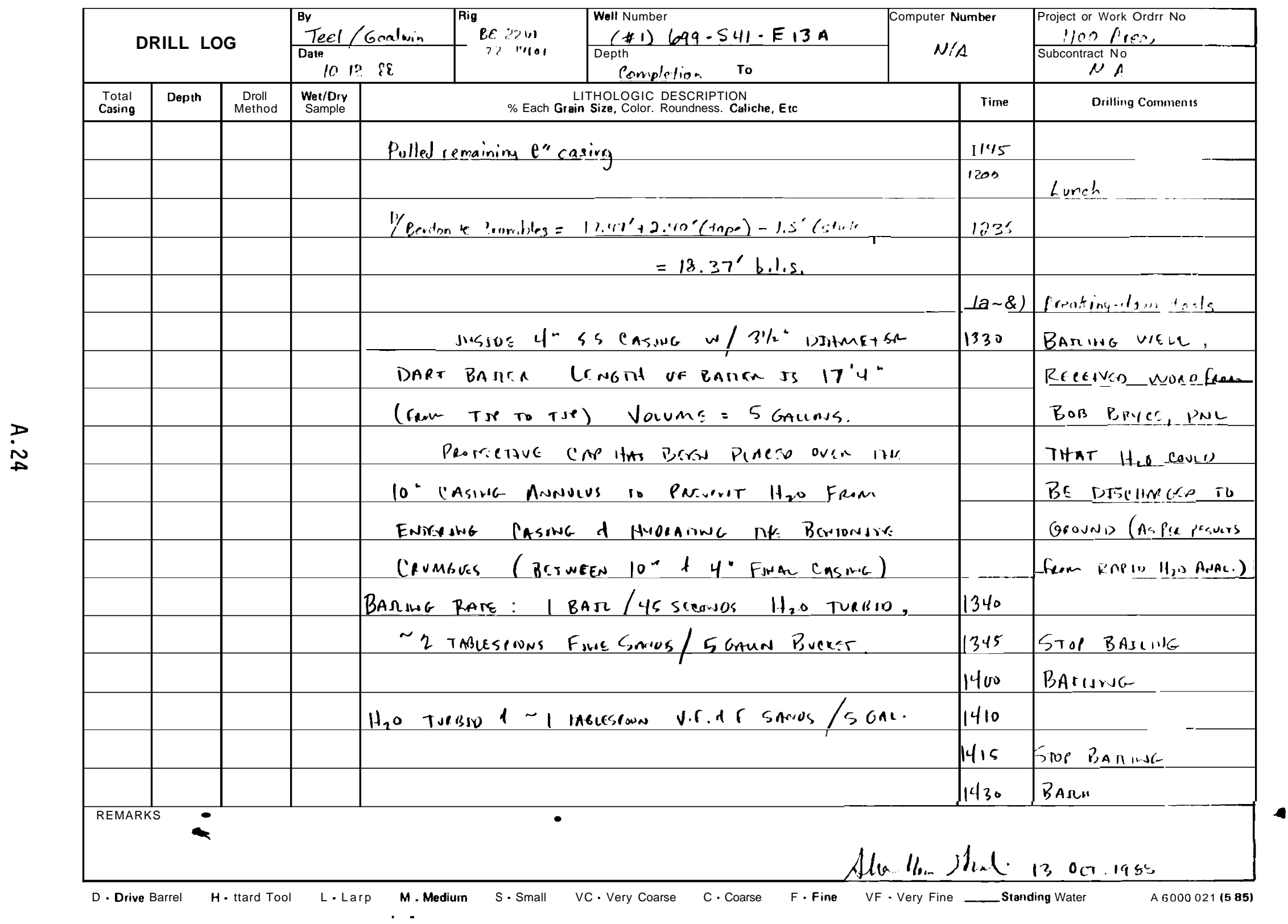




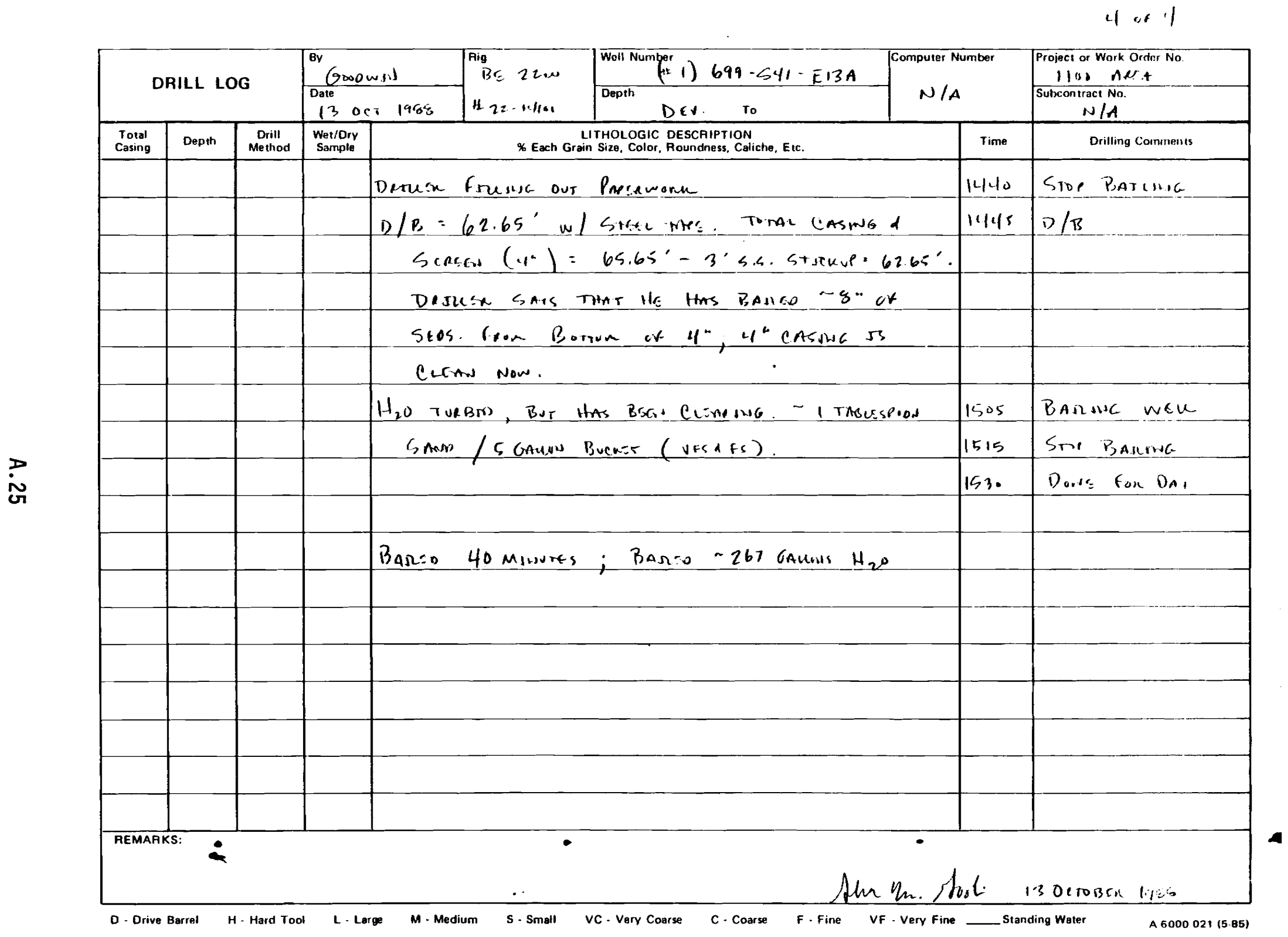


1 or 3

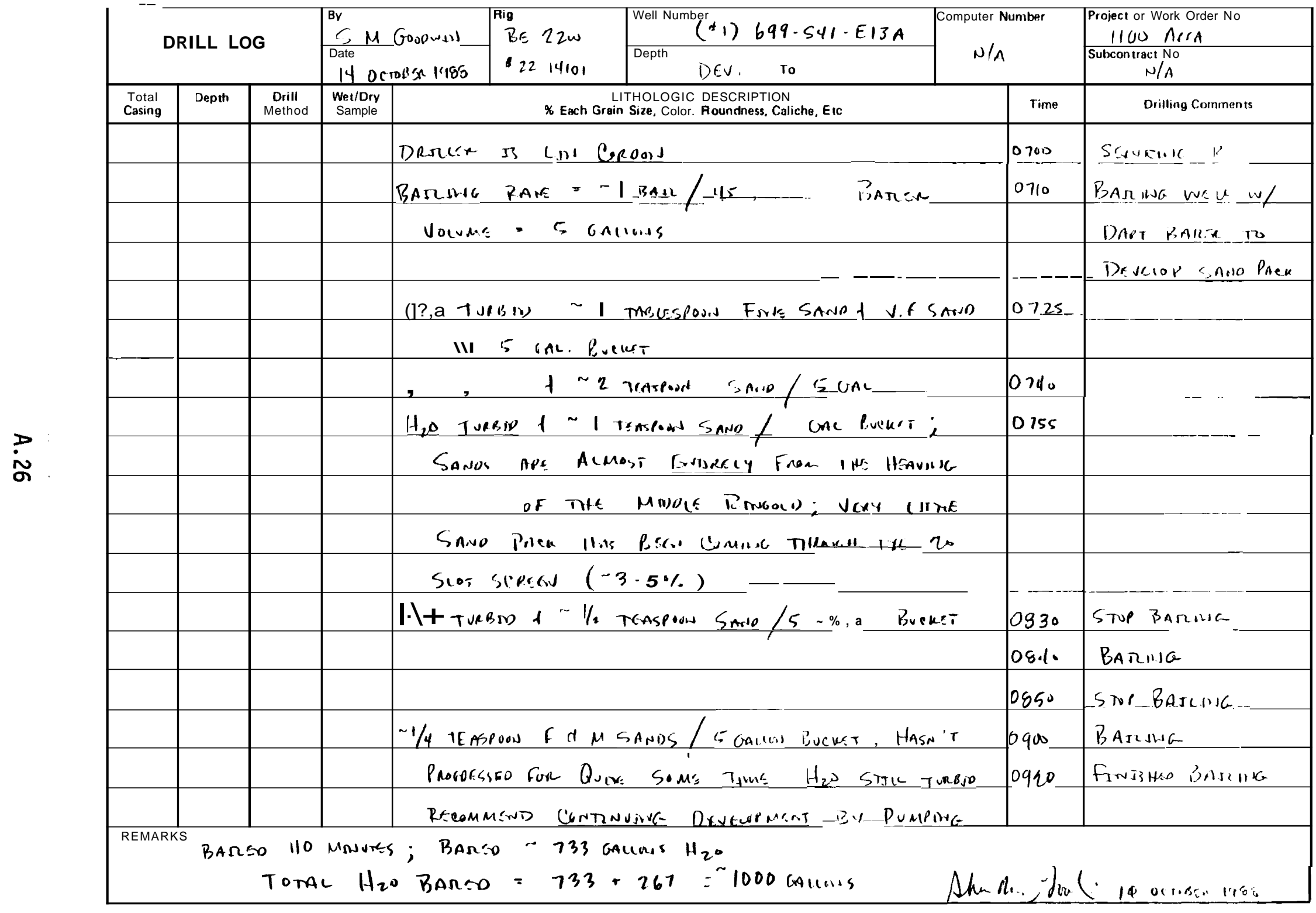

D. Drive Barrel H. Hard Tool L. Large M. Medium S. Small VC. Very Coarse C. Coarse F. Fine VF. Very Fine Sta $n$ d i n g Water $\quad$ A 6000021 (5.85) 
2 of 3

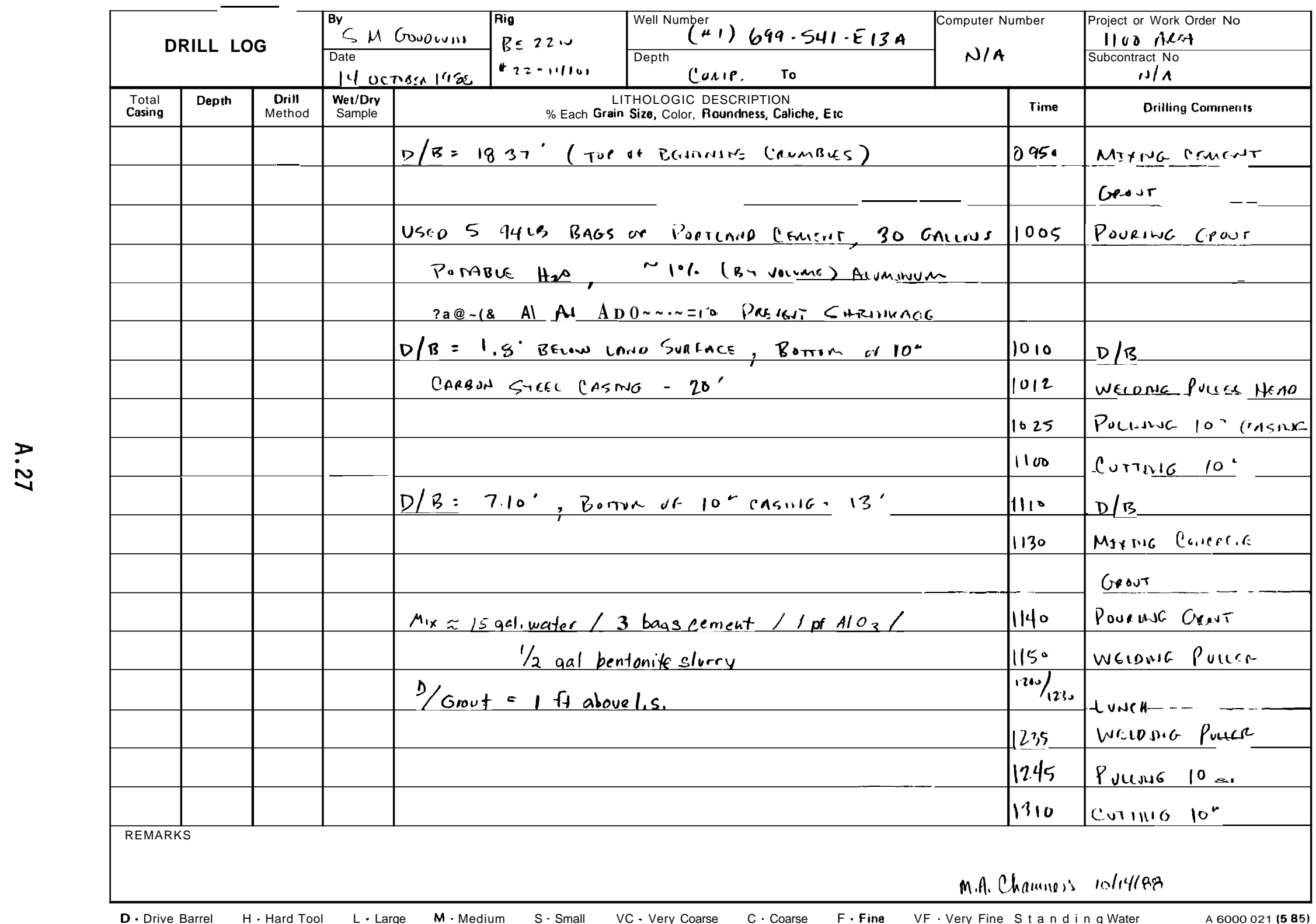




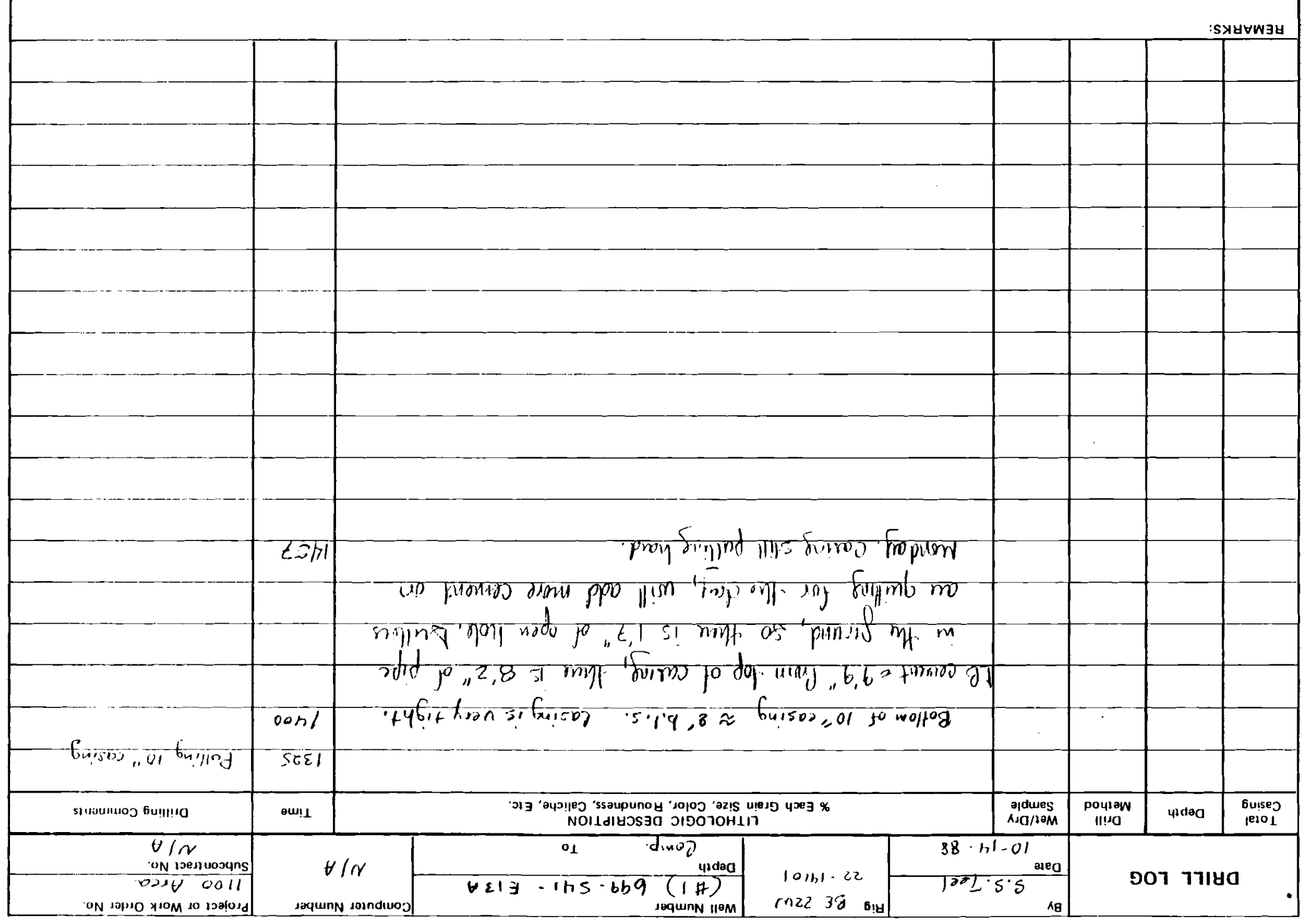

$$
\text { efuE }
$$




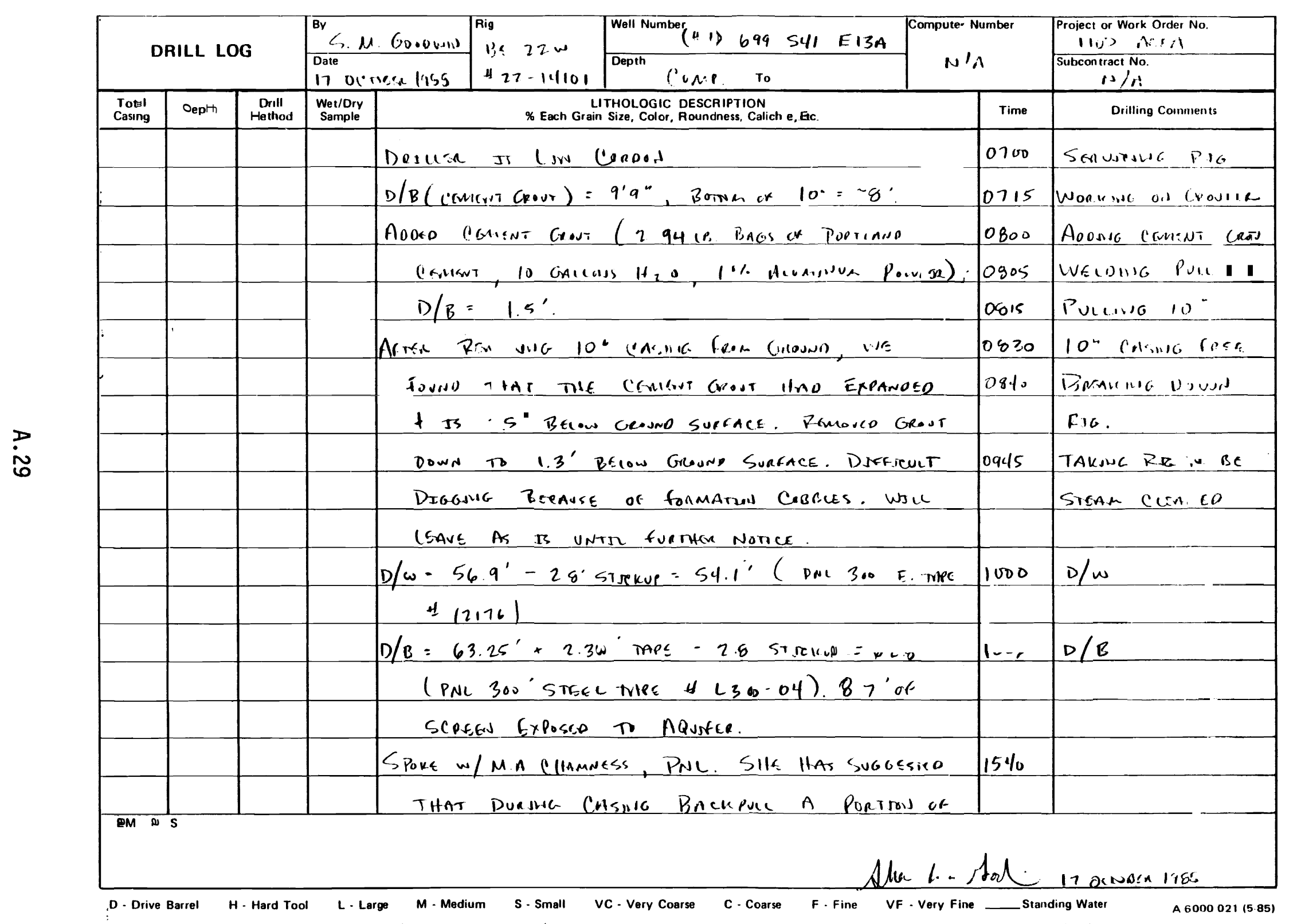




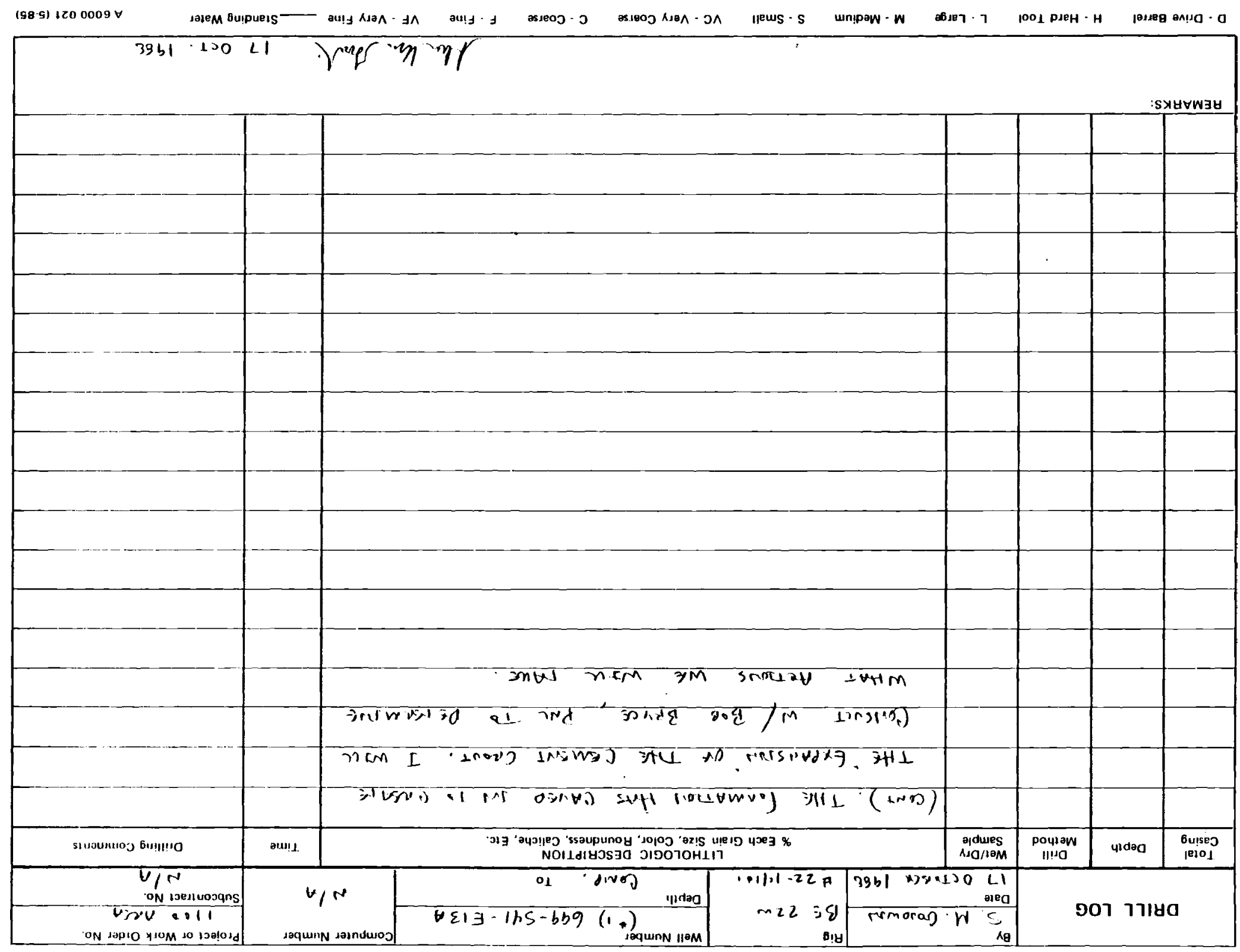


(98.9) $120 \cdot 0009 \forall$

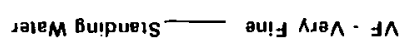

au! . .

as

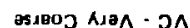

กews - $S$

wnipaw - w $06187 \cdot 7$

1001 P $28 \mathrm{H} \cdot \mathrm{H}$

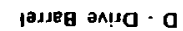

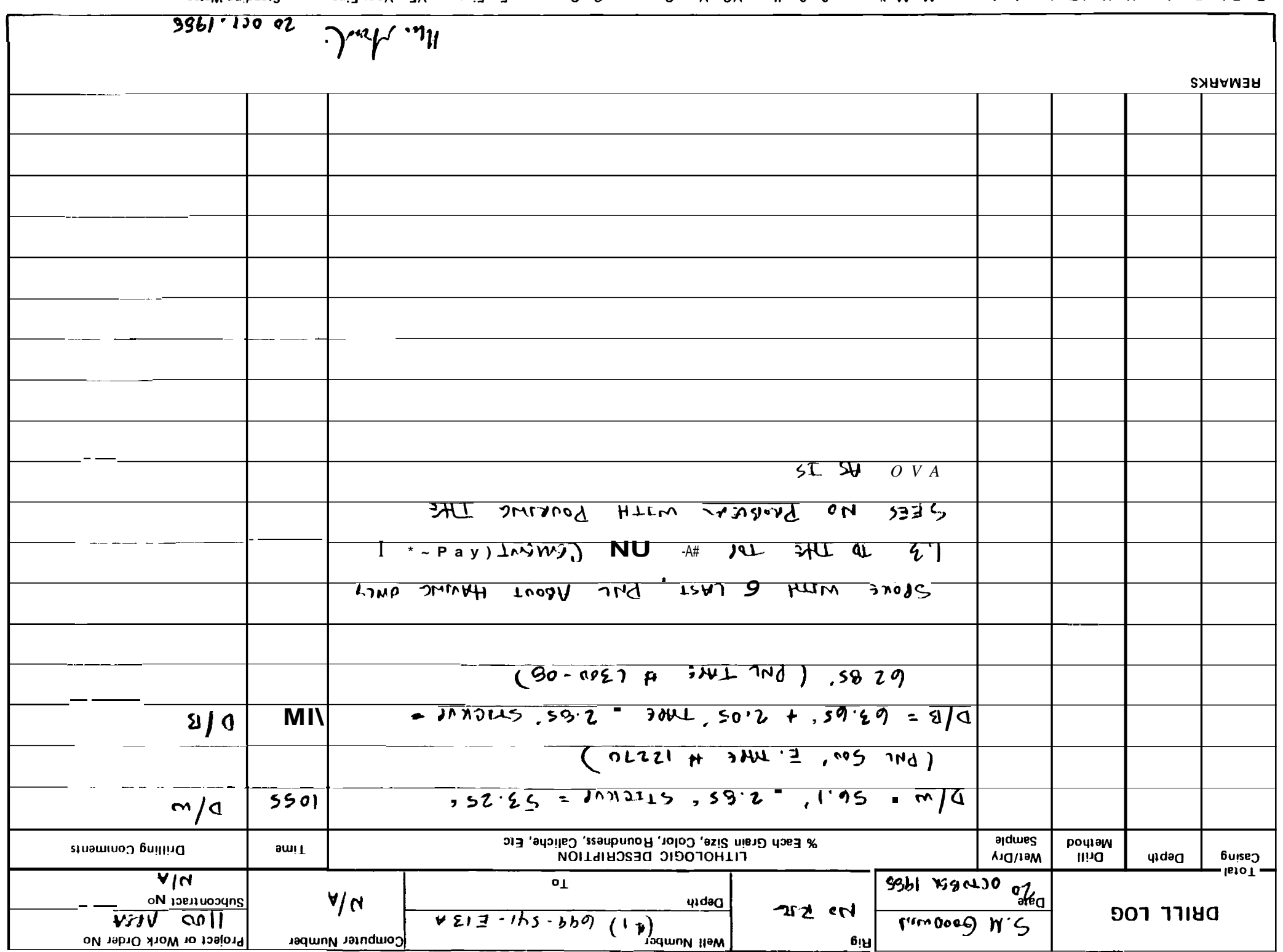




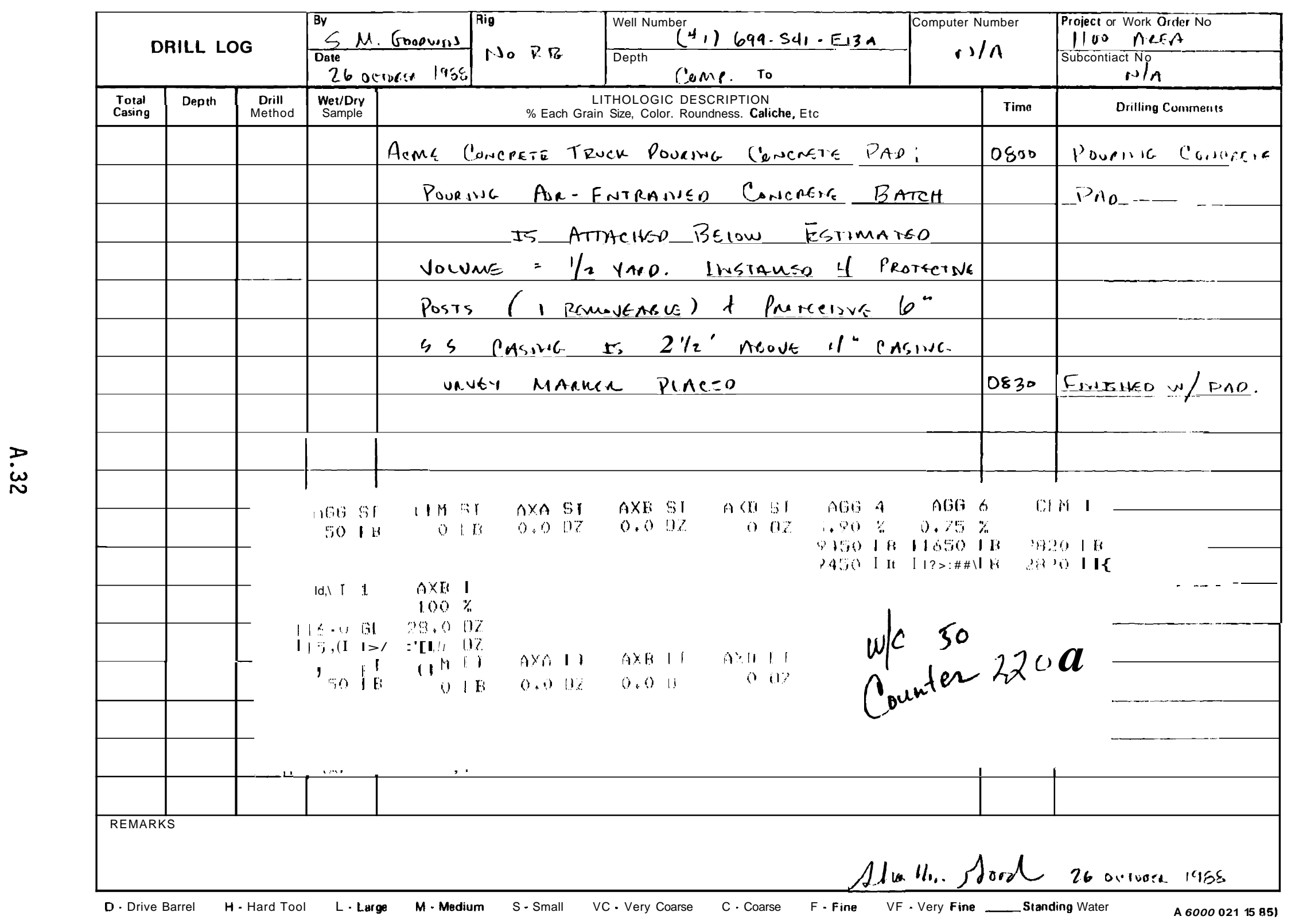


lor 1

\begin{tabular}{|c|c|c|c|c|c|c|c|c|}
\hline \multicolumn{3}{|c|}{ DRILL LOG } & \multicolumn{2}{|c|}{$\begin{array}{l}\text { Bv } S \text { M Govowis } \\
\text { Date } \\
28 \text { ocrussenlitos }\end{array}$} & $\operatorname{trig}_{0} \mathrm{R}_{0}$ & 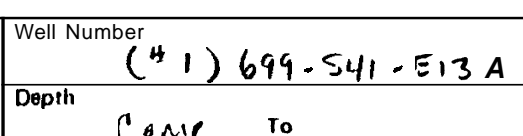 & $\begin{array}{c}\text { Computer Number } \\
\text { I) } / A\end{array}$ & \multirow{2}{*}{\begin{tabular}{|} 
Project or Work Order No \\
1100 nerA \\
$\begin{array}{c}\text { Subcontract No } \\
\text { No }\end{array} /$ A \\
Ditling Cormments
\end{tabular}} \\
\hline $\begin{array}{c}\text { Total } \\
\text { Casing }\end{array}$ & Depth & $\begin{array}{l}\text { Drill } \\
\text { Method }\end{array}$ & 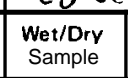 & & \multicolumn{2}{|c|}{ 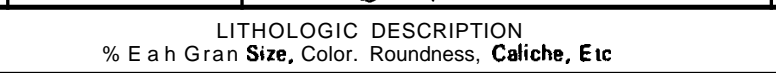 } & \begin{tabular}{|l|l} 
& rime \\
\end{tabular} & \\
\hline & & & & Penceo & wek o & weu cnp & 1025 & - \\
\hline & & & & & & & & L- - \\
\hline & & & & & & & & \\
\hline & & & & & & & & \\
\hline & & & & & & & & \\
\hline & & & & & & & & \\
\hline & & & & & & & & \\
\hline & & & & & & & & \\
\hline & & & & & & & & \\
\hline & & & & & & & & \\
\hline & & & & & & & & \\
\hline & & & & & & & & \\
\hline & & & & & & & & \\
\hline & & & & & & & & \\
\hline & & & & & & & & \\
\hline & & & & & & & & \\
\hline & & & & & & & & \\
\hline REMAR & & & & & & & & \\
\hline & & & & & & sh & m...s.unt & 28 octuBs. $116 \%$ \\
\hline
\end{tabular}


1 or 3

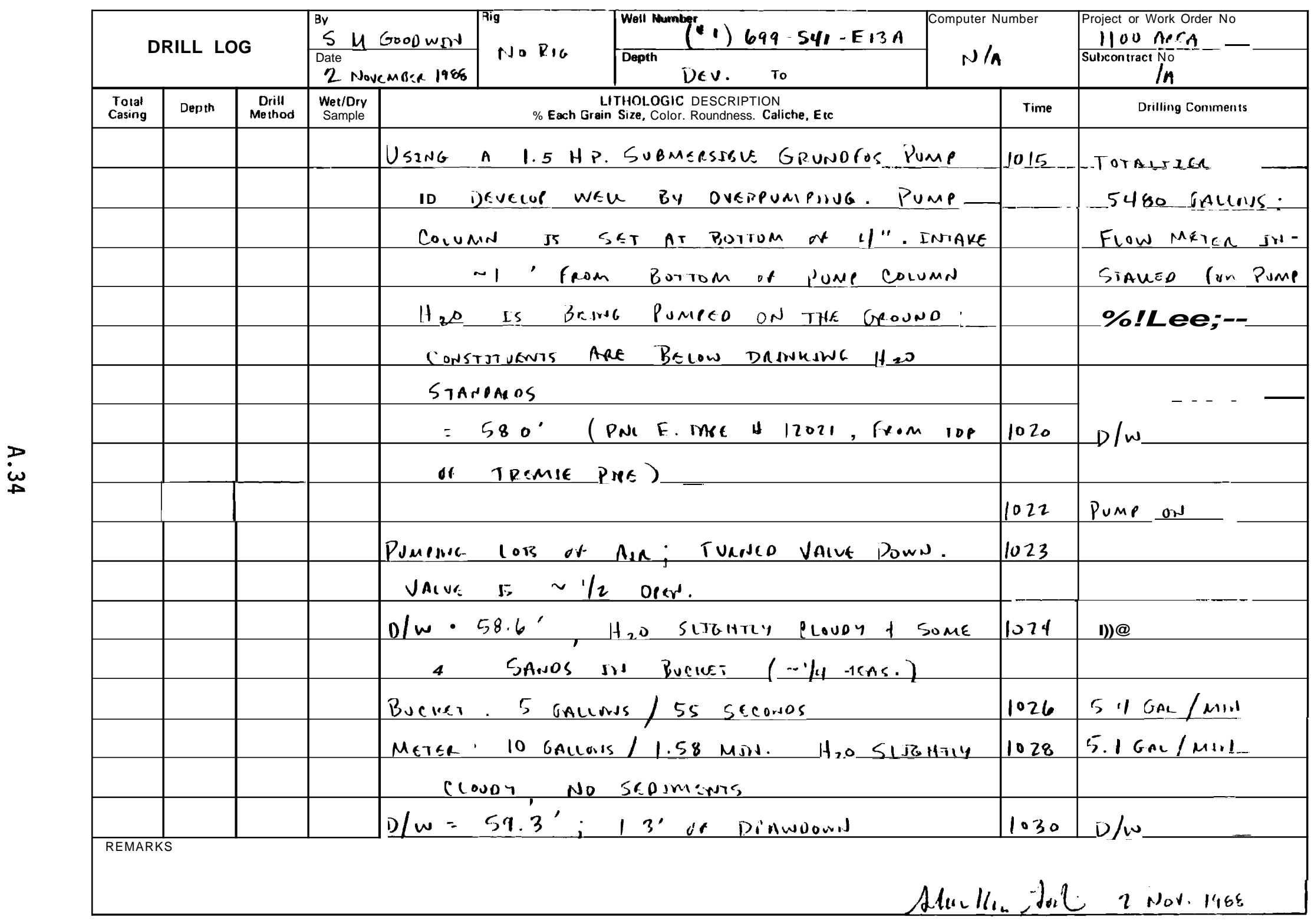

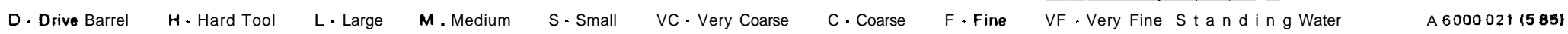




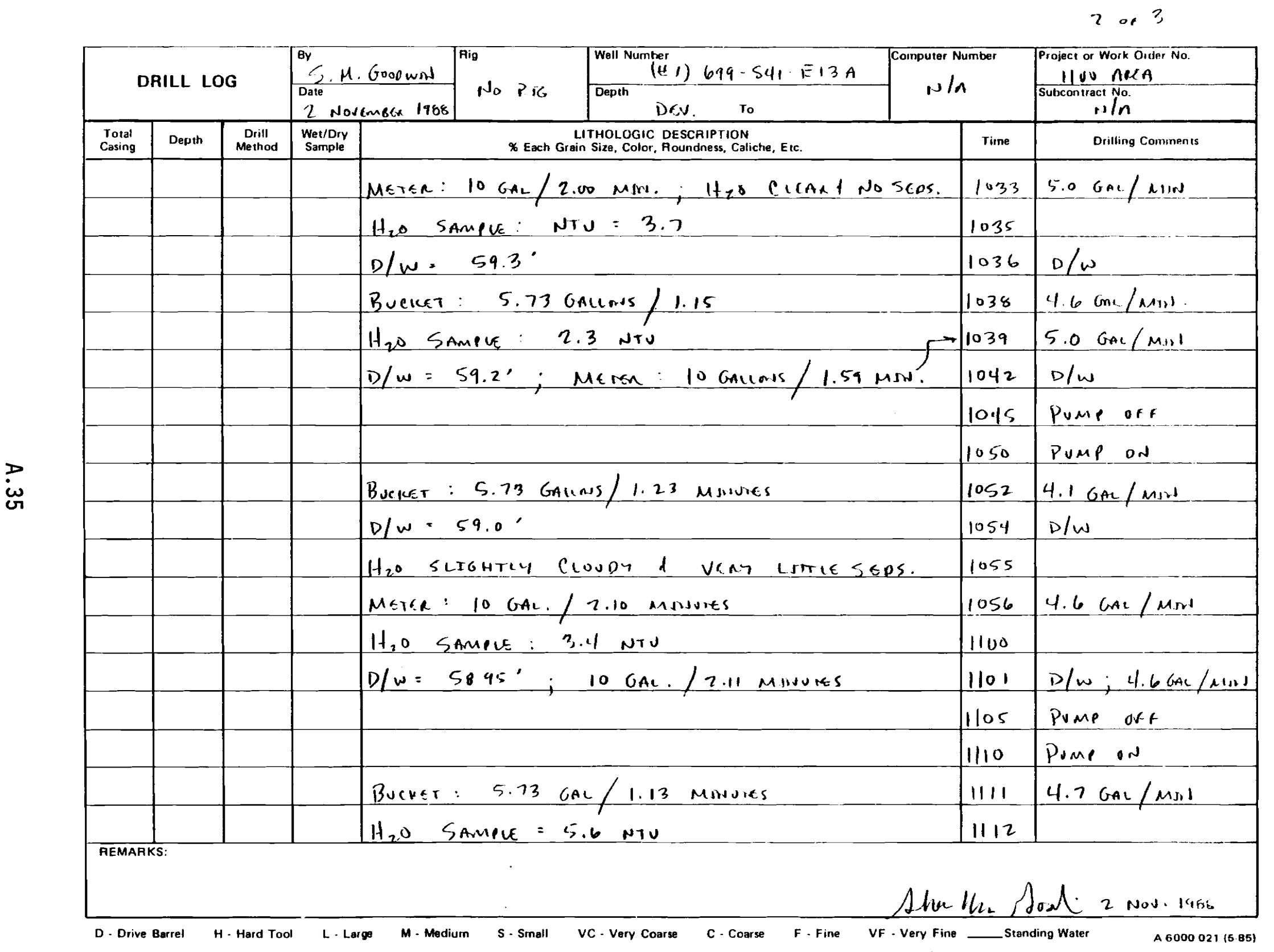


3 of 3

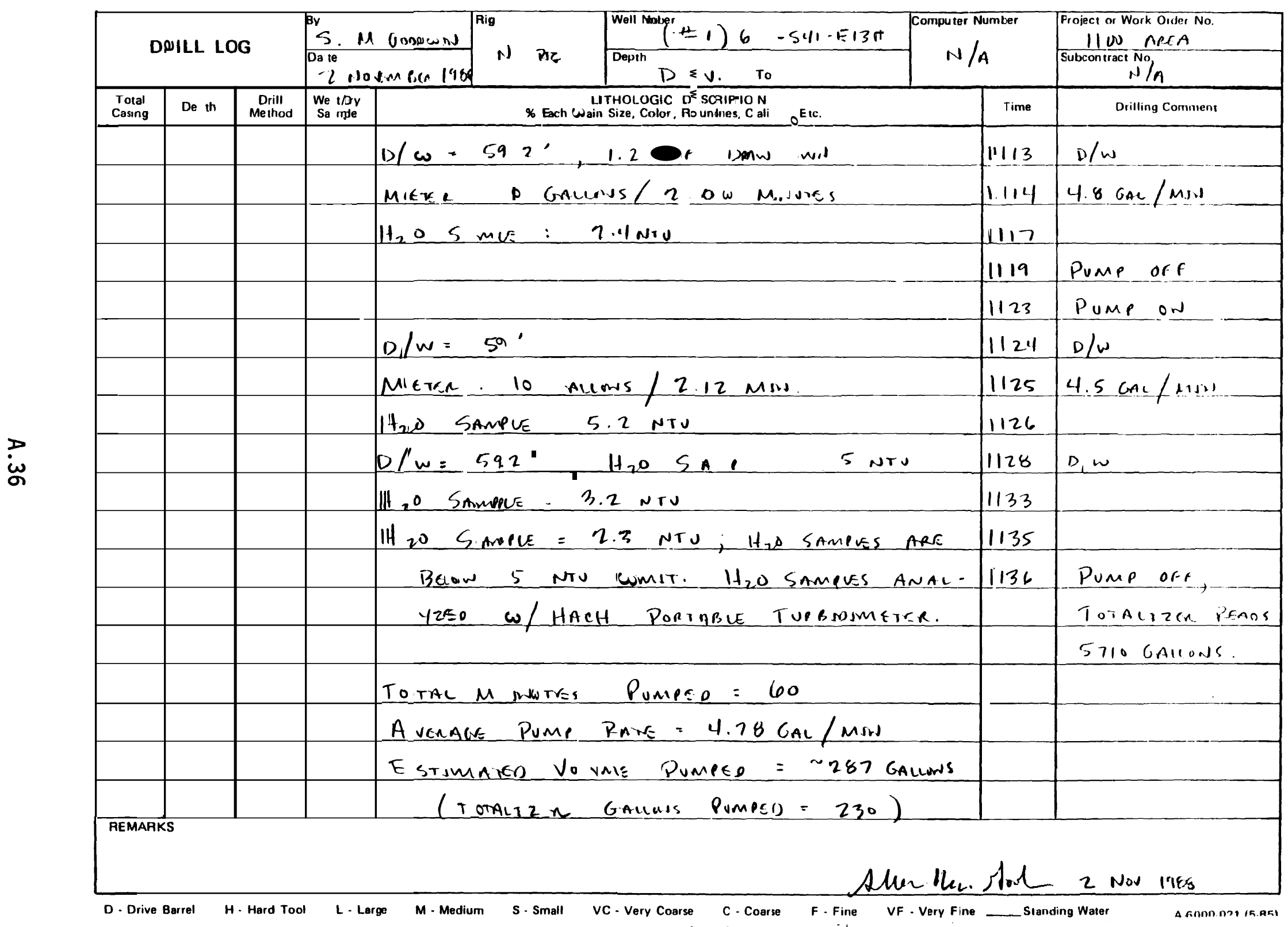


1 of 2

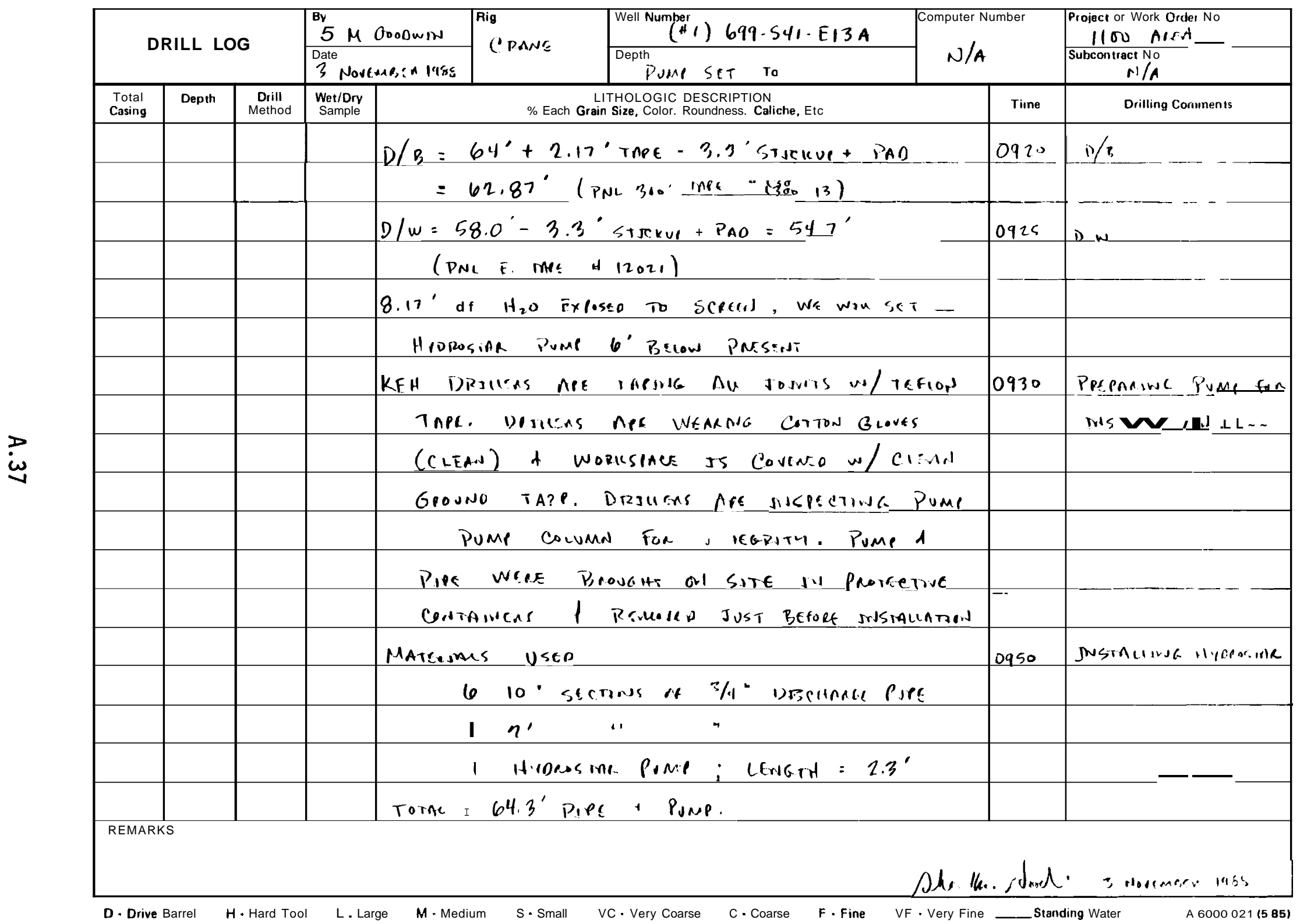




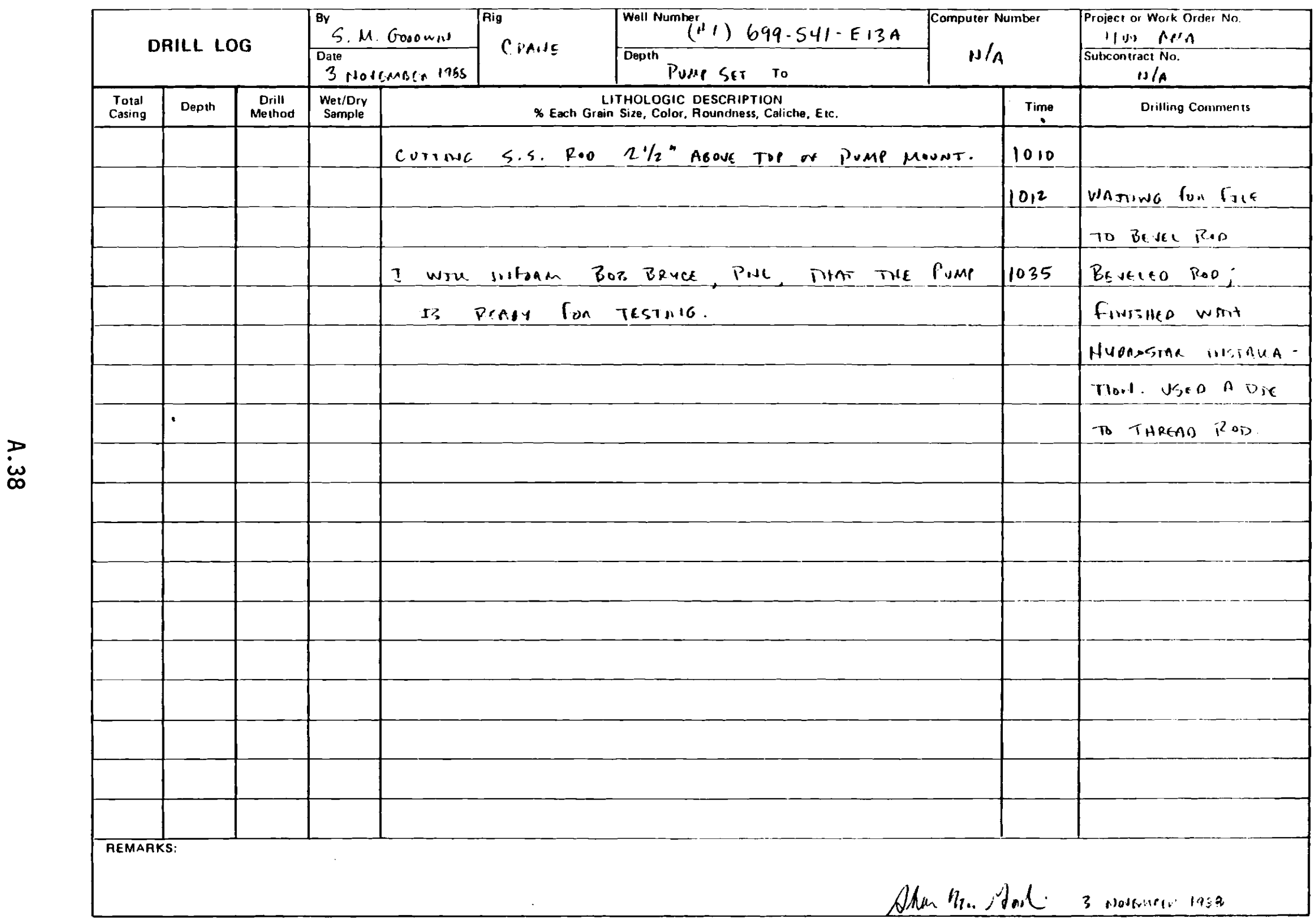

D. Drive Barret H- Hard Tool L-Large M-Medium S-Small VC-Very Coarse C-Coarse F-Fine VF-Very Fine -Standing Water A6000 021 (5.85) 


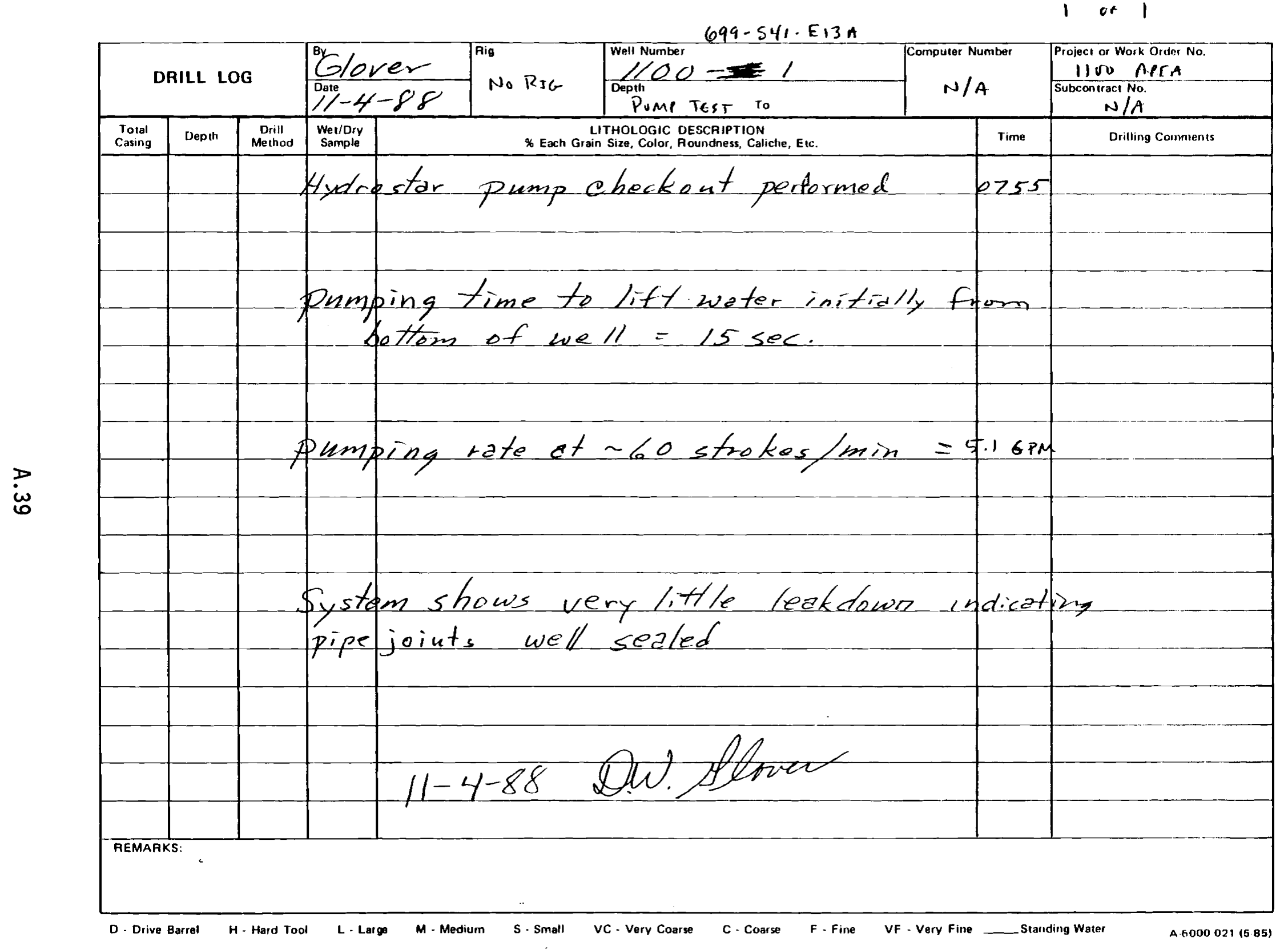


APPENDIX B

GEOPHYSICAL AND GEOLOGIC LOGS FOR WELL 2 (699-S40-E14) 


\section{APPENDIX B}

\section{GEOPHYSICAL AND GEOLOGIC LOGS FOR WELL 2 (699-S40-E14)}

This appendix contains the We11 Completion/Inspection Report, as-built diagram, notes from the sampling pump install ation, the natural gamma log, and the geologists' logs for well $2(699-\mathrm{S} 40-\mathrm{E} 14)$ in the 1100 Area. 
Procedure P-6

Attachment 1

Page 1 of 1

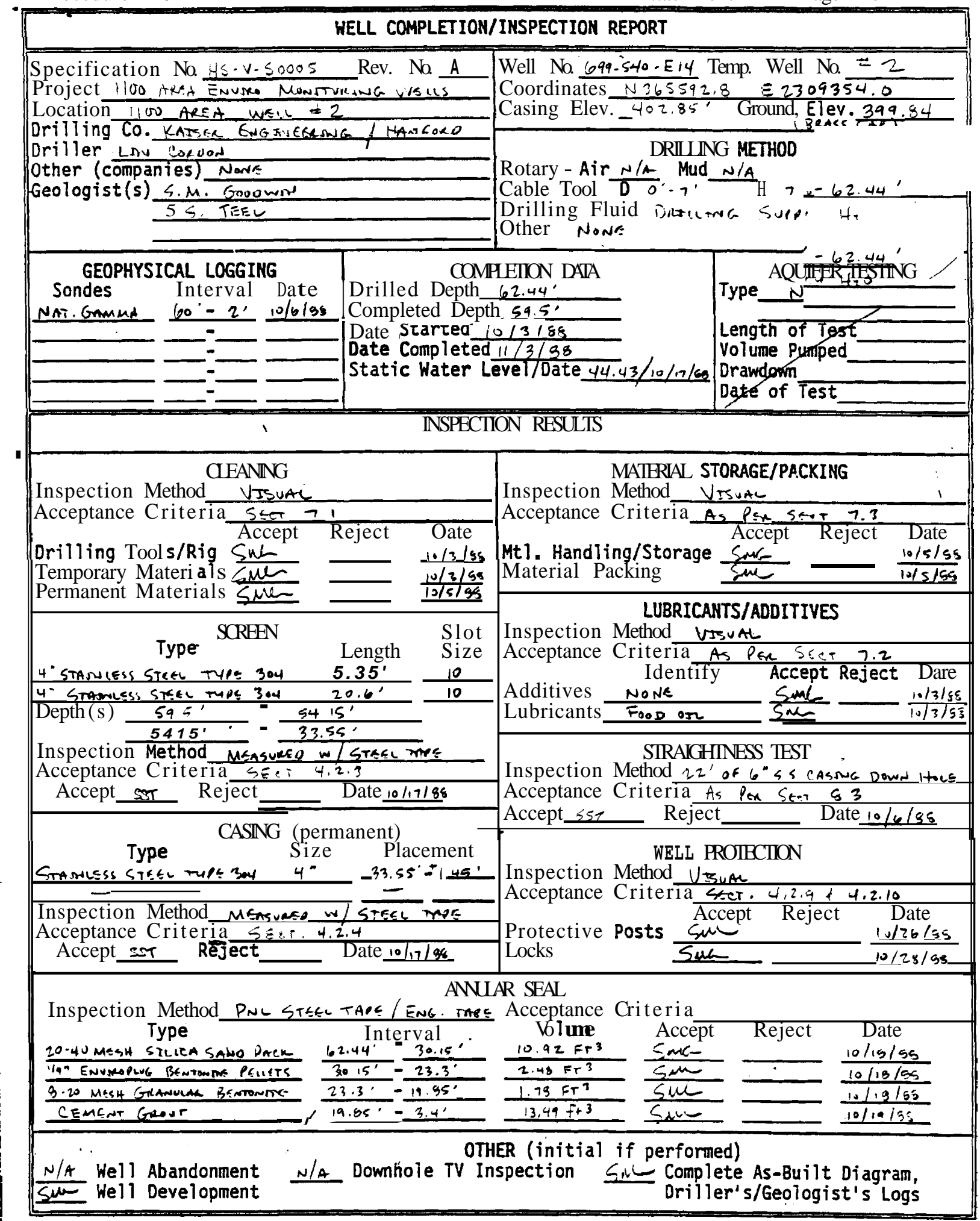

Reviowes by vih)ne slem 11-7-88

For all blanks mark N/A if not applicable. 


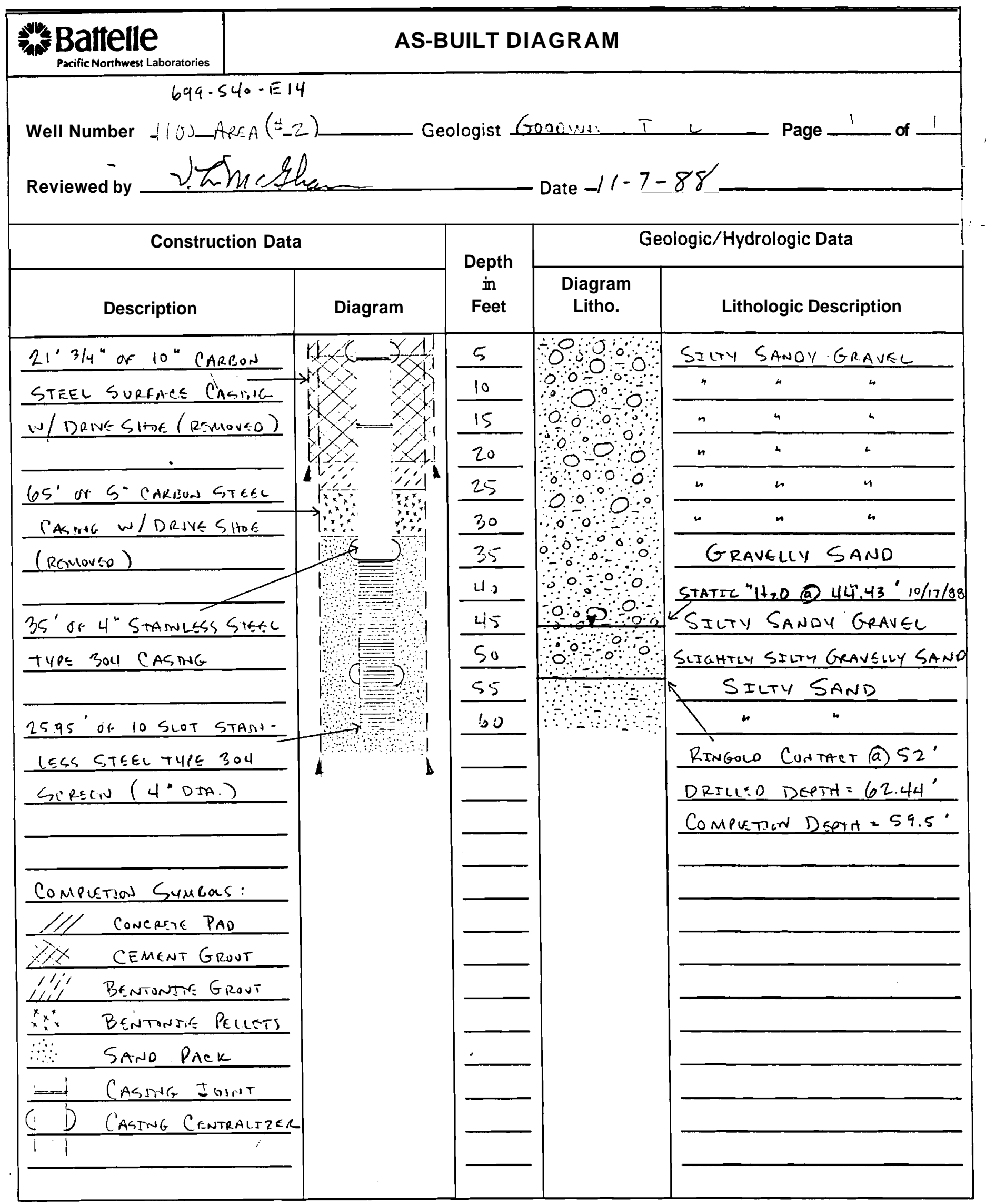


SAMPLING PUMP INSTALLATION IN

GROUND-WATER MONITORING WELLS

site: 1100 AREA

Monitoring Well Number: $(\# 2) 699-540-E 14$

Depth to Water: $47.10^{\circ}$

Depth to Bottom: $59.12^{\prime}$ Reported Depth to Bottom: $59.5^{\prime}$

Pump Type: Positive Displacement Piston

Pump Model: Hydrostar HS-8001

Date Installed: $11 / 3 / 88$

Installed By: KEH: OLN AMus, LOUIS WATKSWS; LENNY COROON

Pump Discharge Pipe Description:

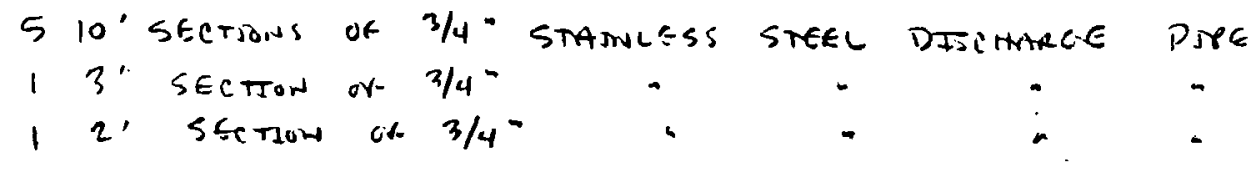

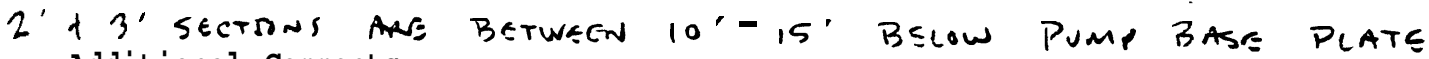
Additional Comments:

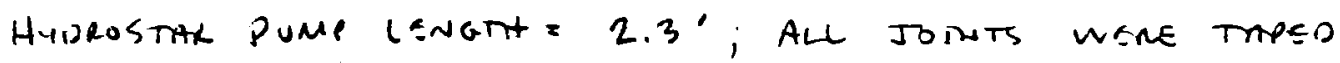

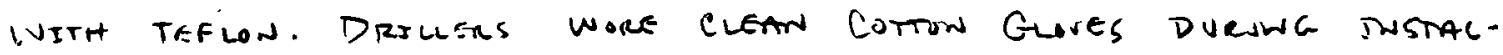
LATTON.

Sketch of Pump Instal lation:
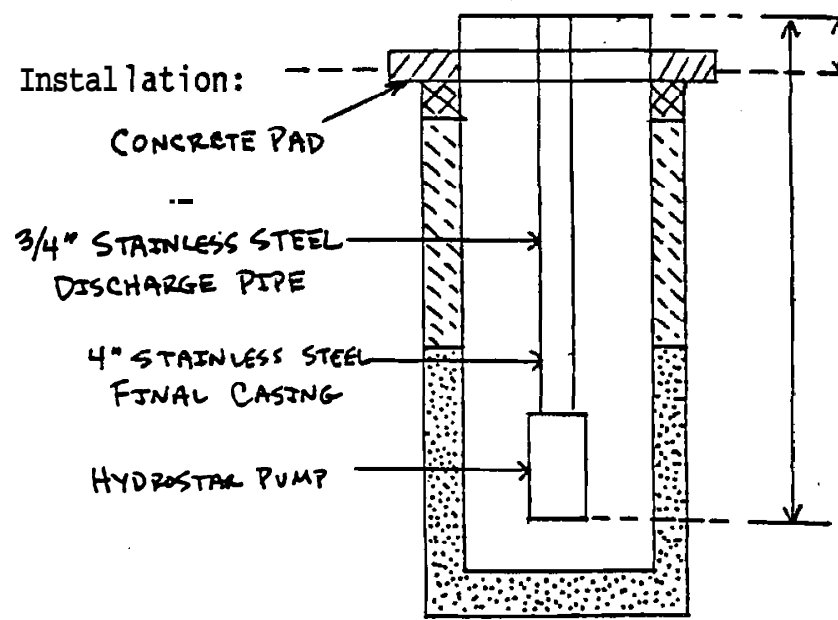

COnCrETE PAD + 6n CASTNG

CONCRETE PAD DASCHARGE PIPE 4" statnvess stee final casting

HYDZOSTAR PUMP

T OT DIScinage PIPG

+ PUMP $=57.3^{\circ}$

BOTTOM OF INTAKE $=53.58^{\circ}$

Completed By: Whanth, Joord:

Date: $11 / 3 / 88$ 


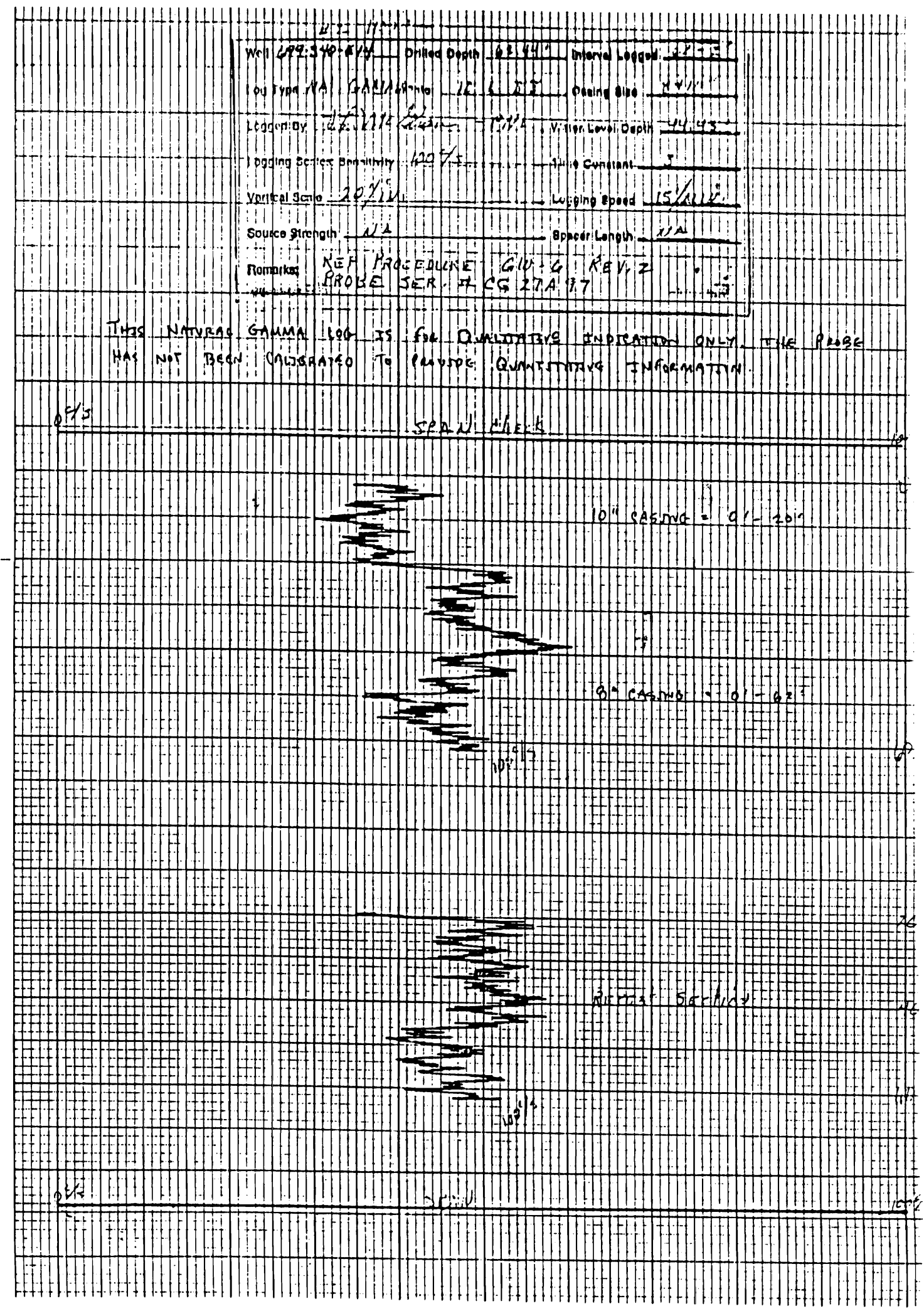

B. 6 
I of 1

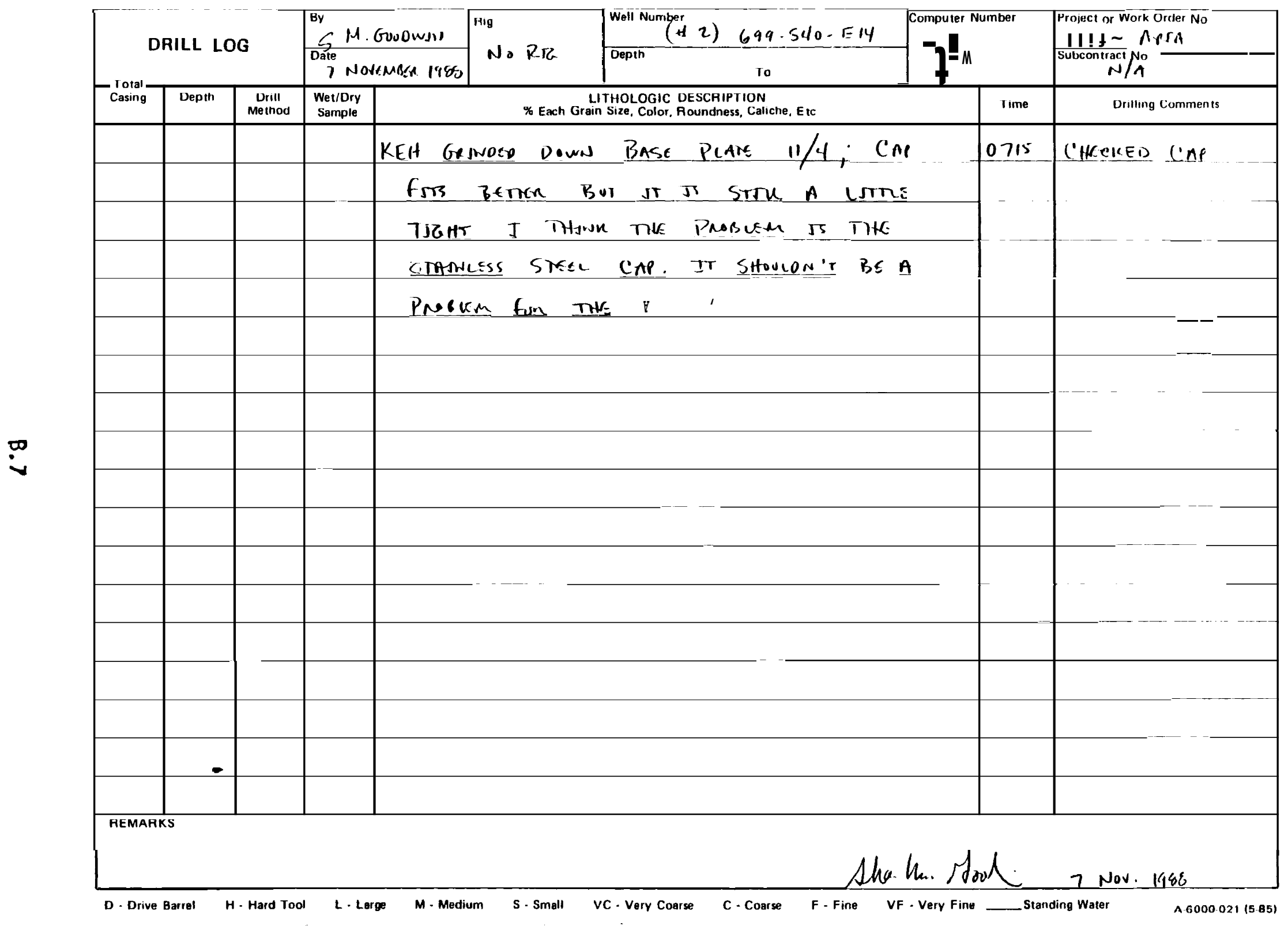


1 of 3

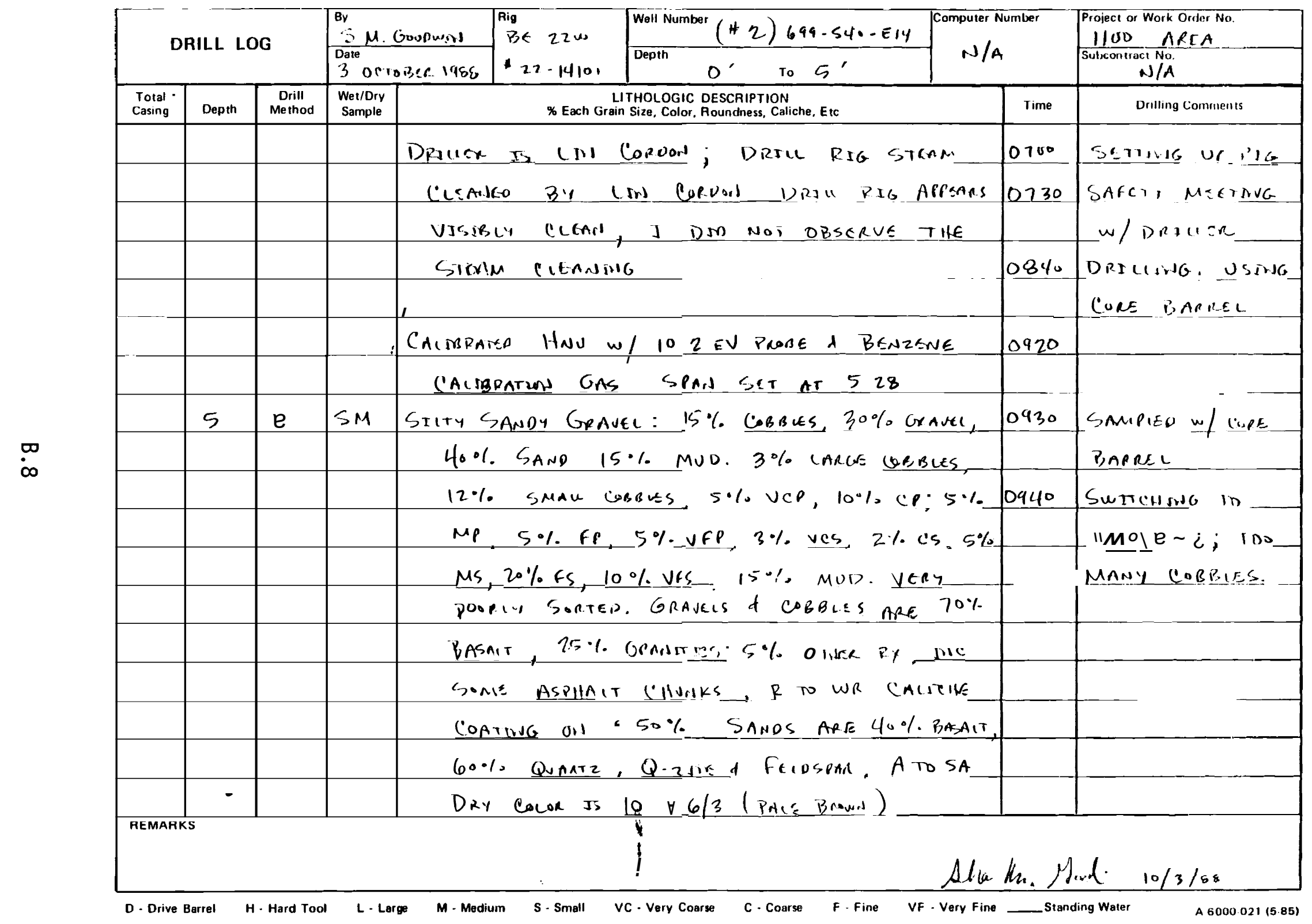




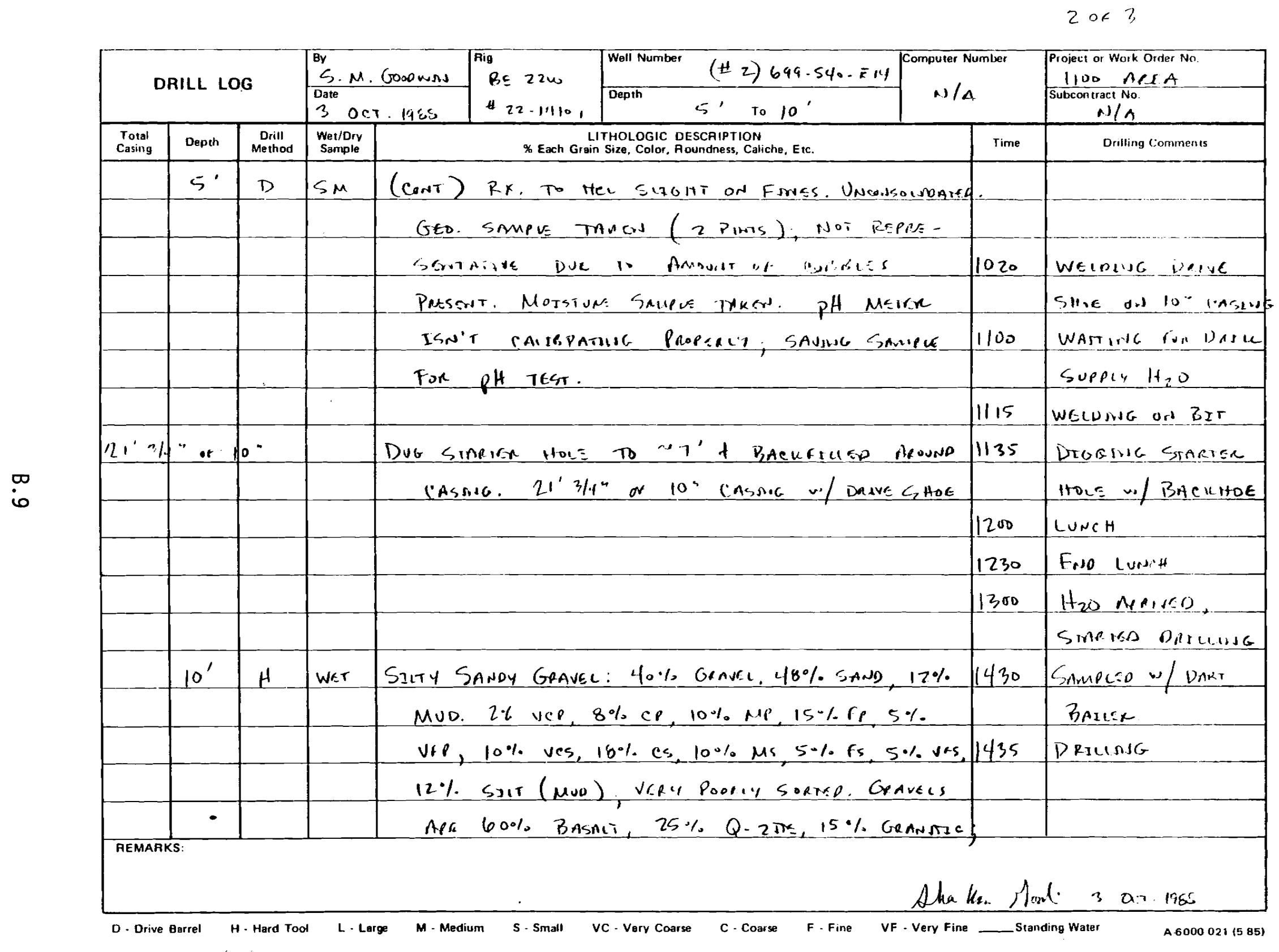


3 or 3

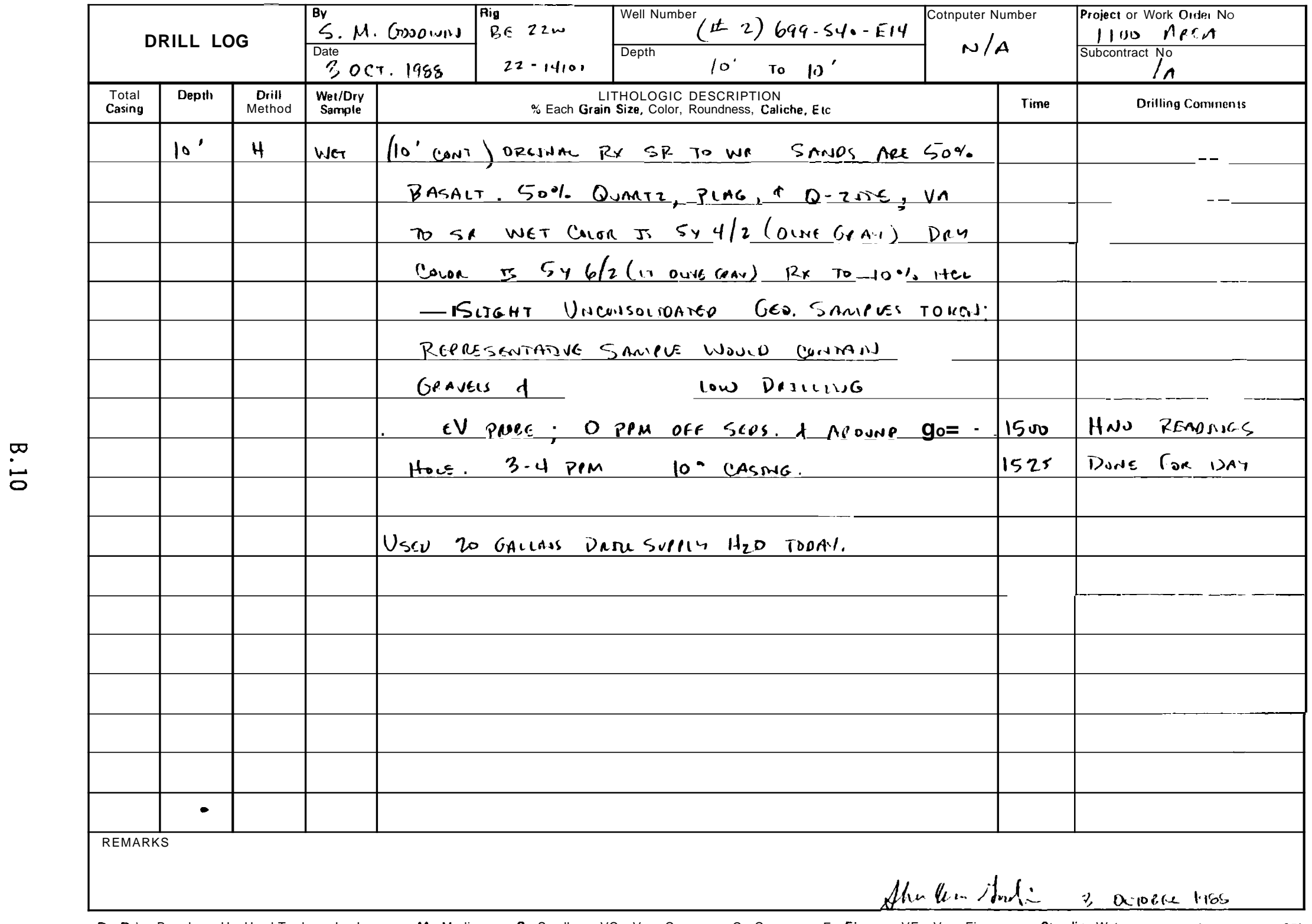


for 4

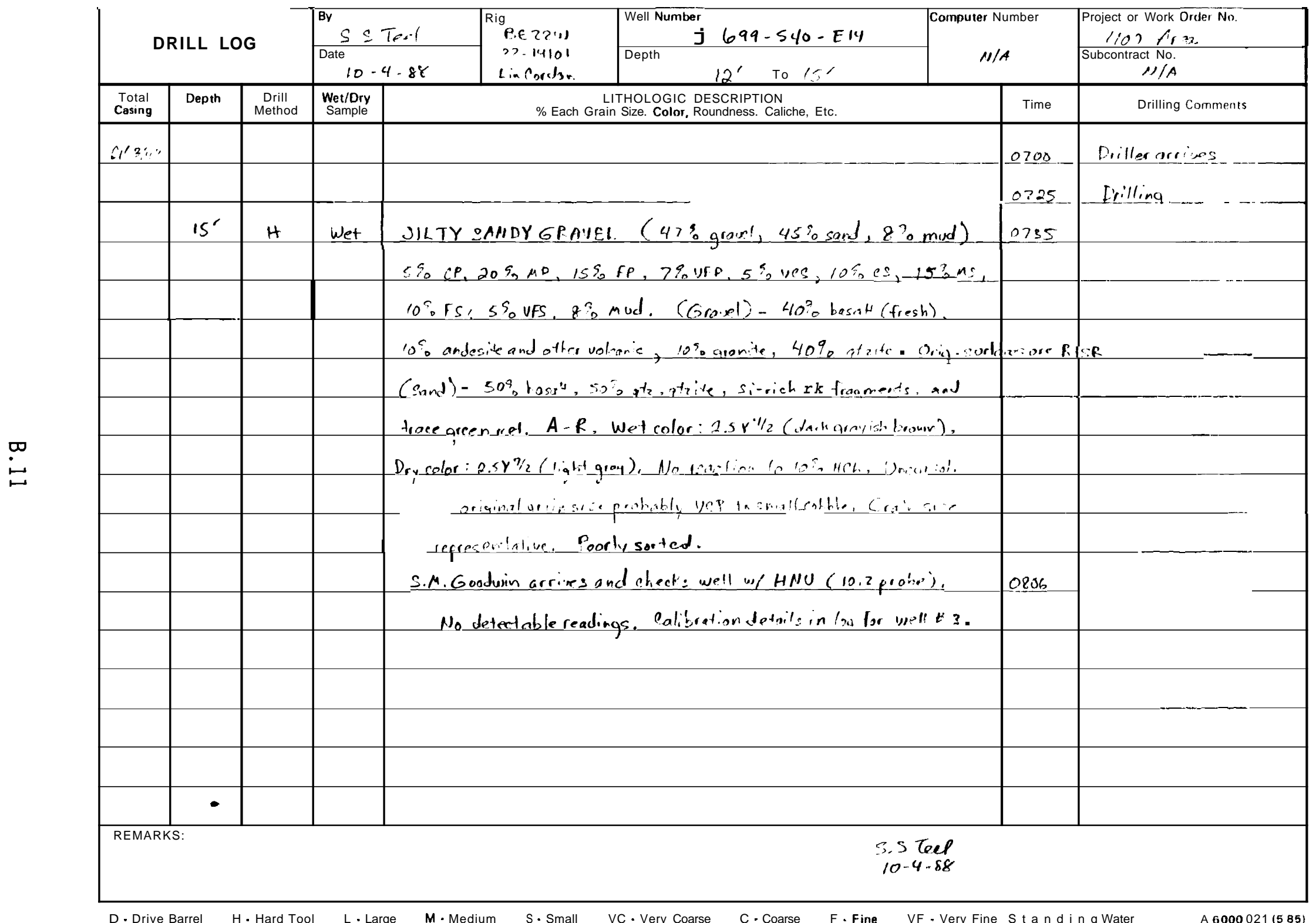


$\therefore 4$

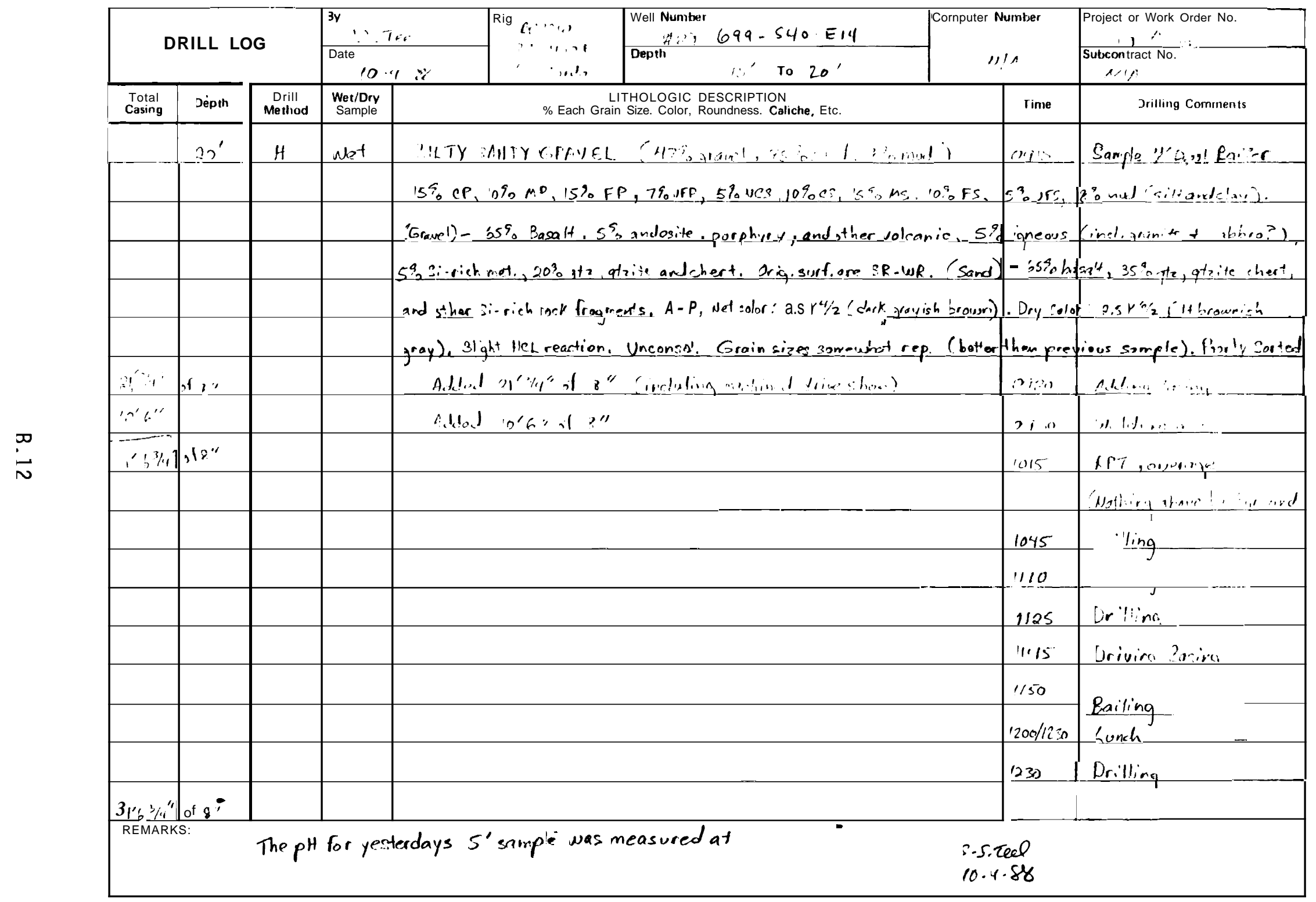

D. Drive Barrel $\quad$ H. Hard Tool L. Large $\quad$ M. Medium $\quad$ S. Small $\quad$ VC. Very Coarse $\quad$ C. Coarse $\quad F$. Fine $\quad$ VF. Very Fine $S t a n d i n g$ Wafer 


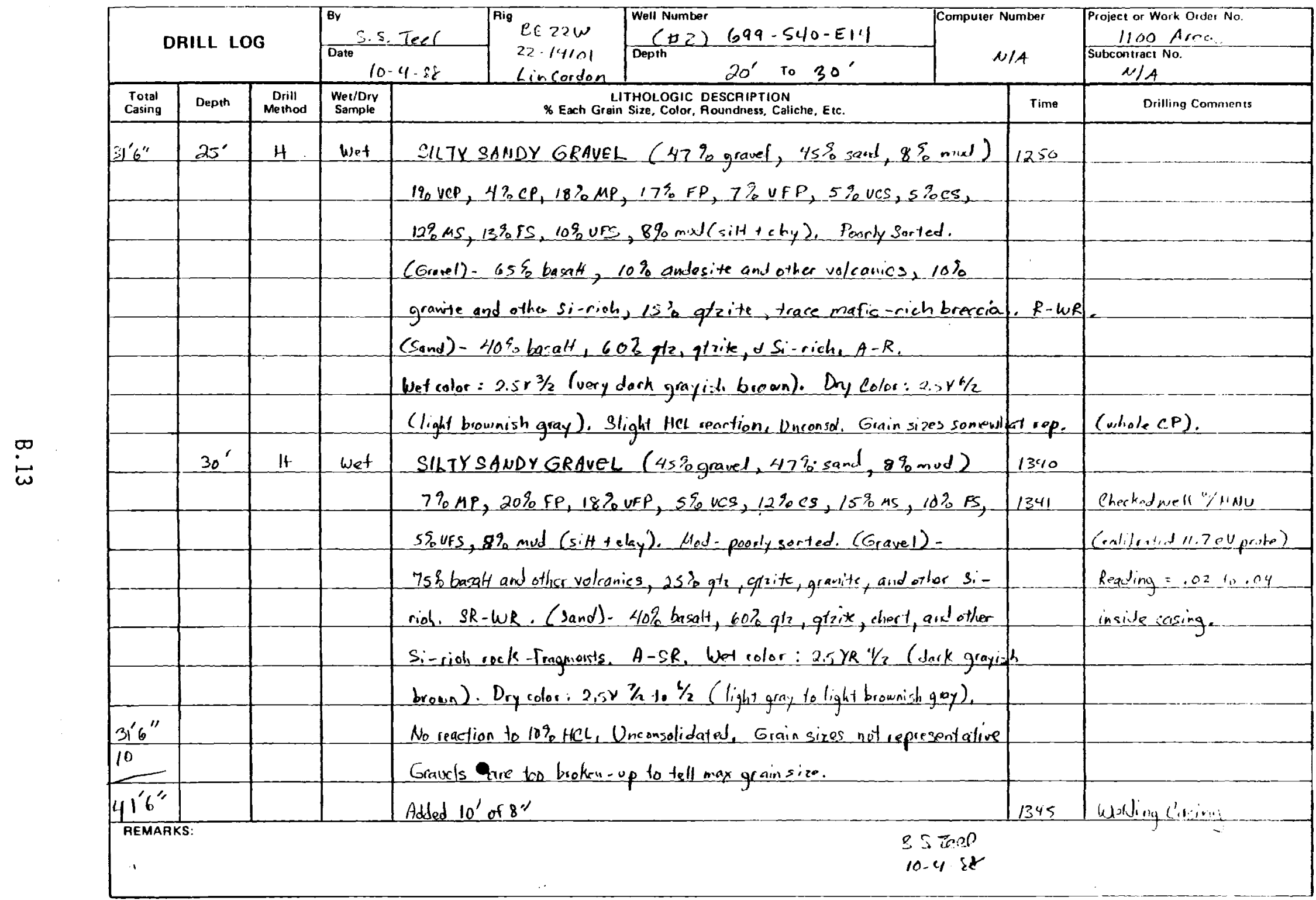

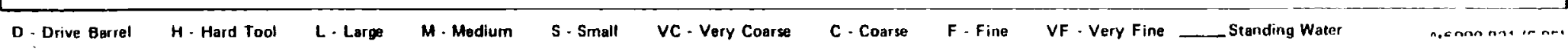




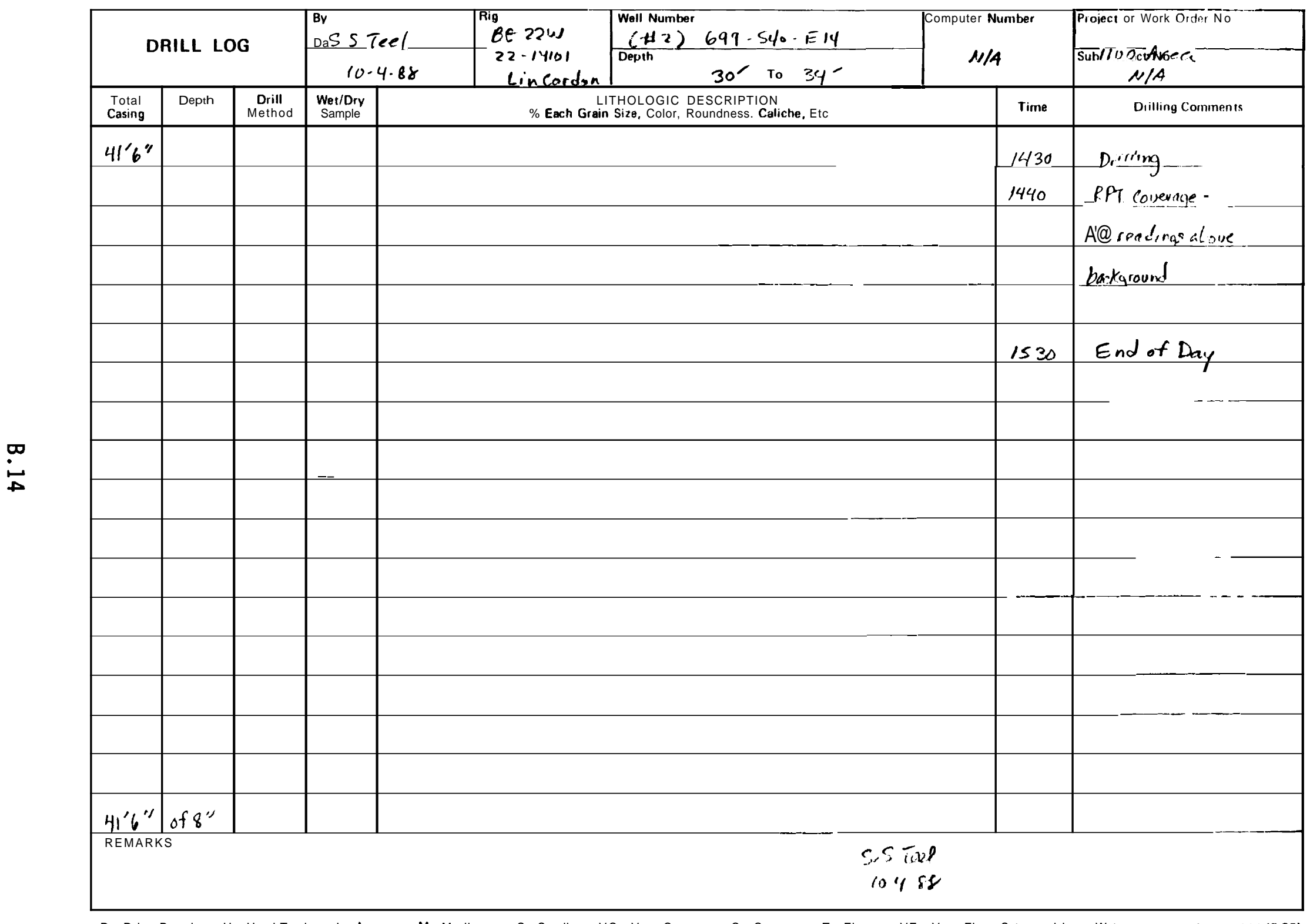

D. Drive Barrel H. Hard Tool L. Large $\quad$ M. Medium S. Small $\quad$ VC. Very Coarse $\quad$ C. Coarse $\quad$ F - Fine $\quad$ VF · Very Fine S t a nd ing Water $\quad$ A6000 021 (5.85) 
1 or 4

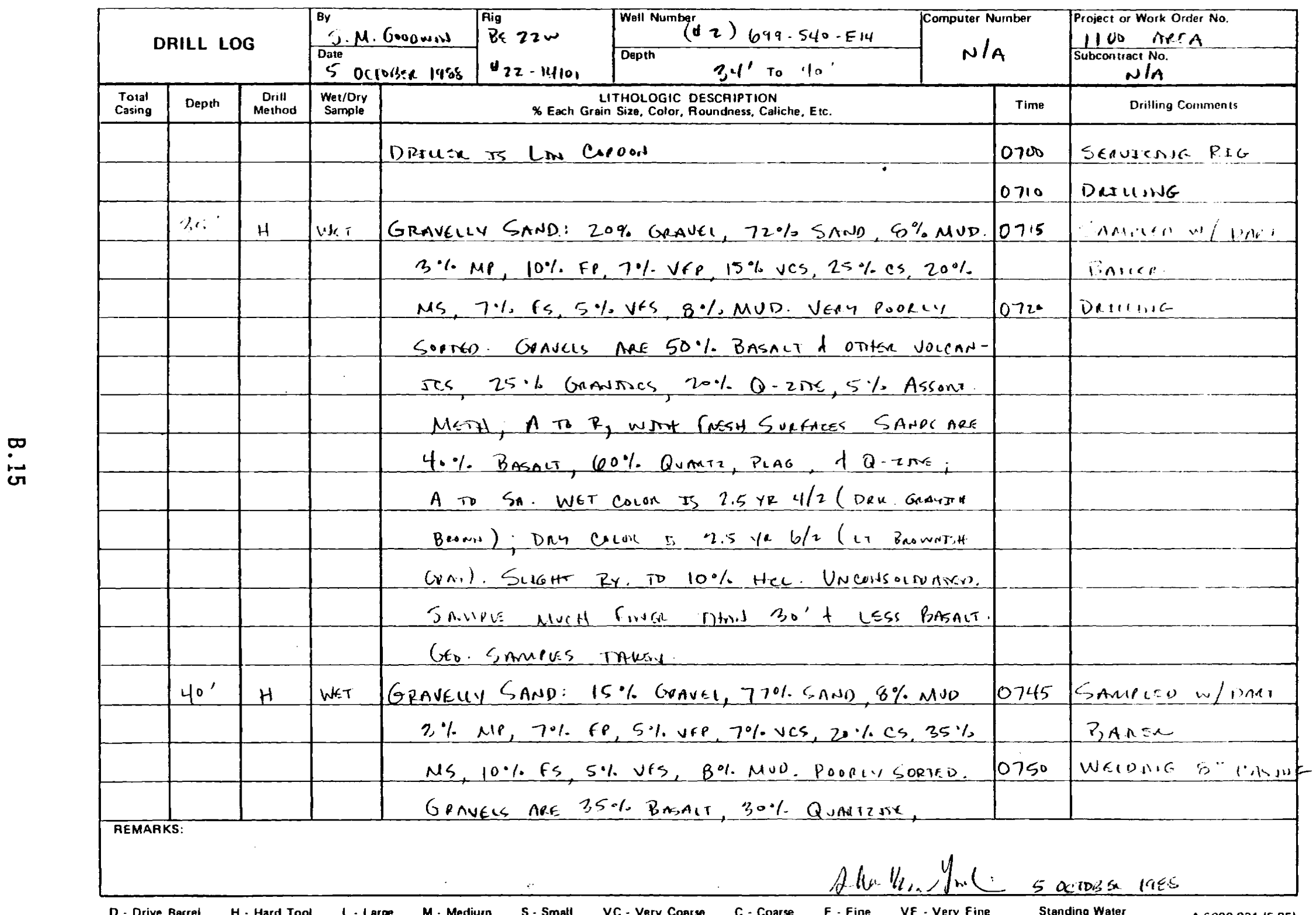




$$
2 \text { or } 4
$$

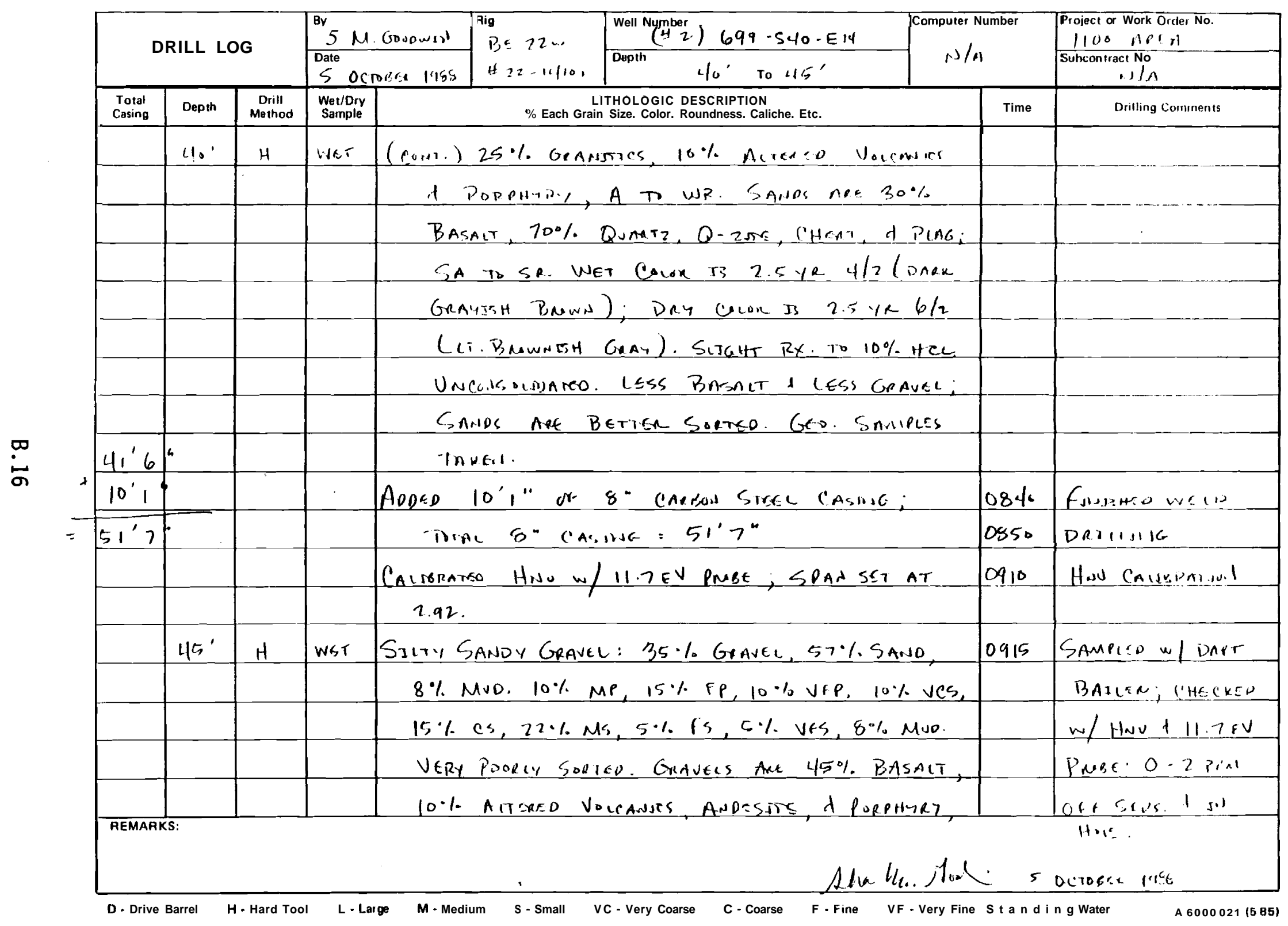


3 or 4

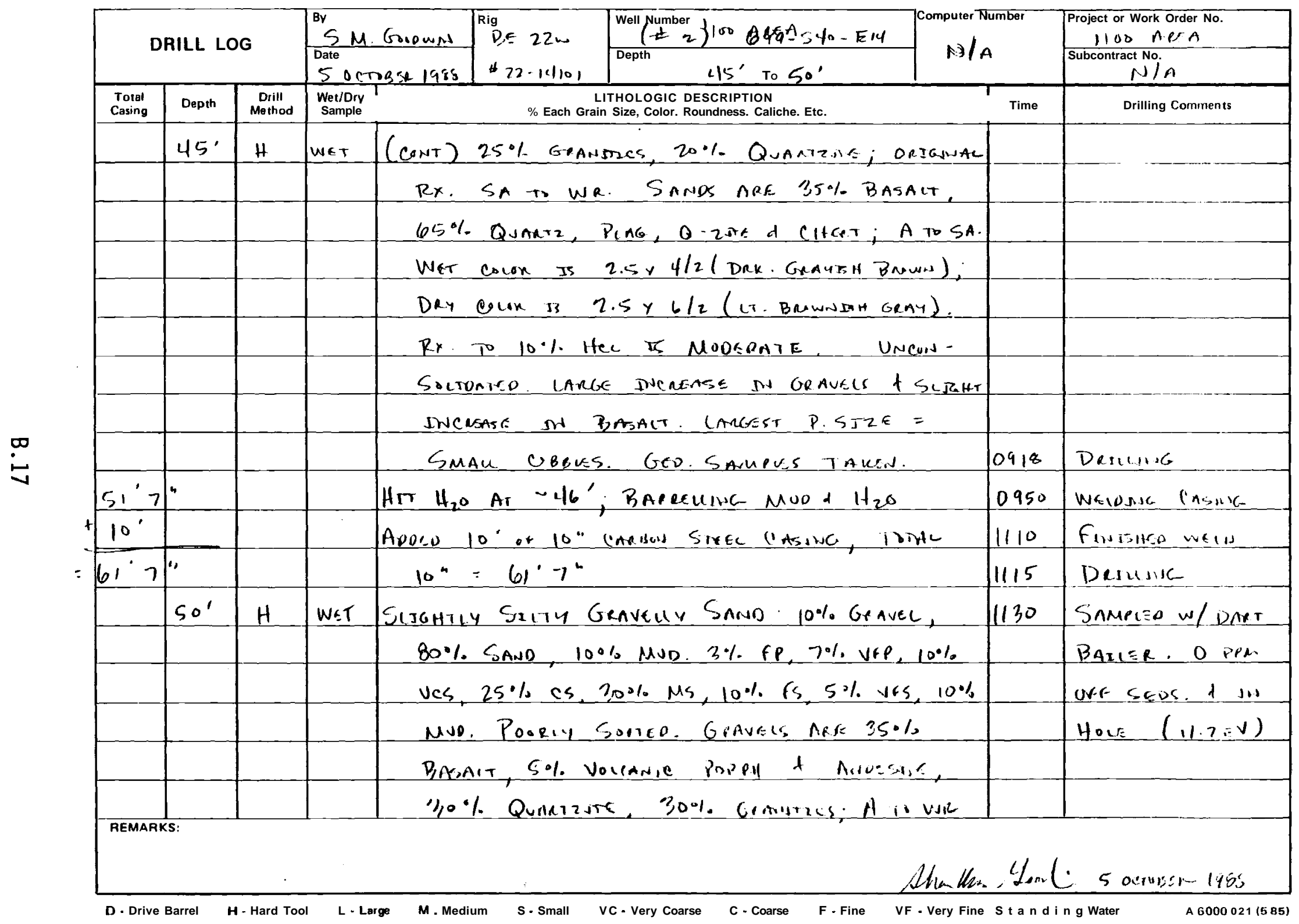




$$
4 \operatorname{or} 4
$$

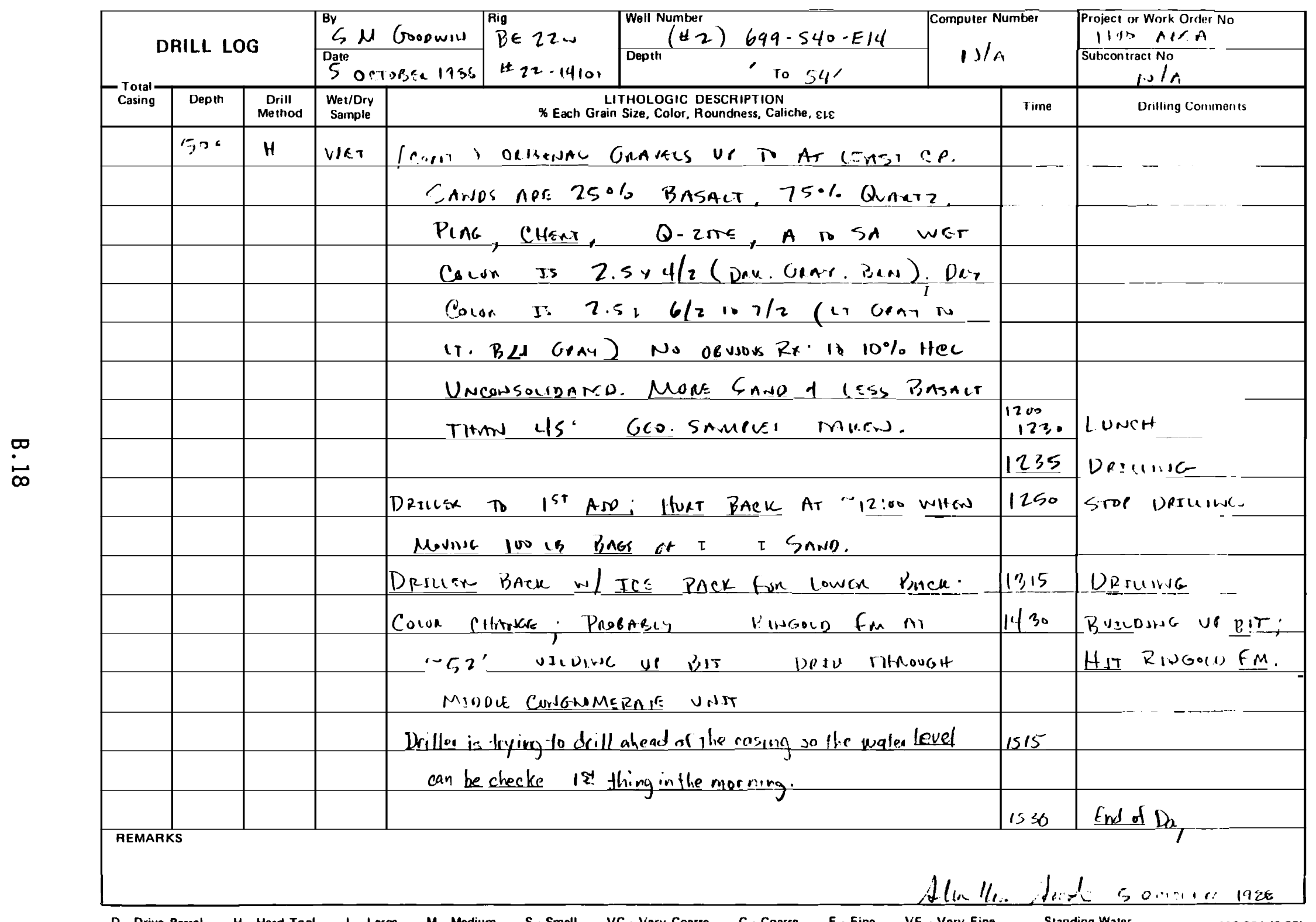

D- Drive Barrel H- Hard Tool L-Large M-Medium S-Small VC-Very Coarse C - Coarse F-Fine VF - Very Fine _-Standing Water 
$\operatorname{lof} 3$

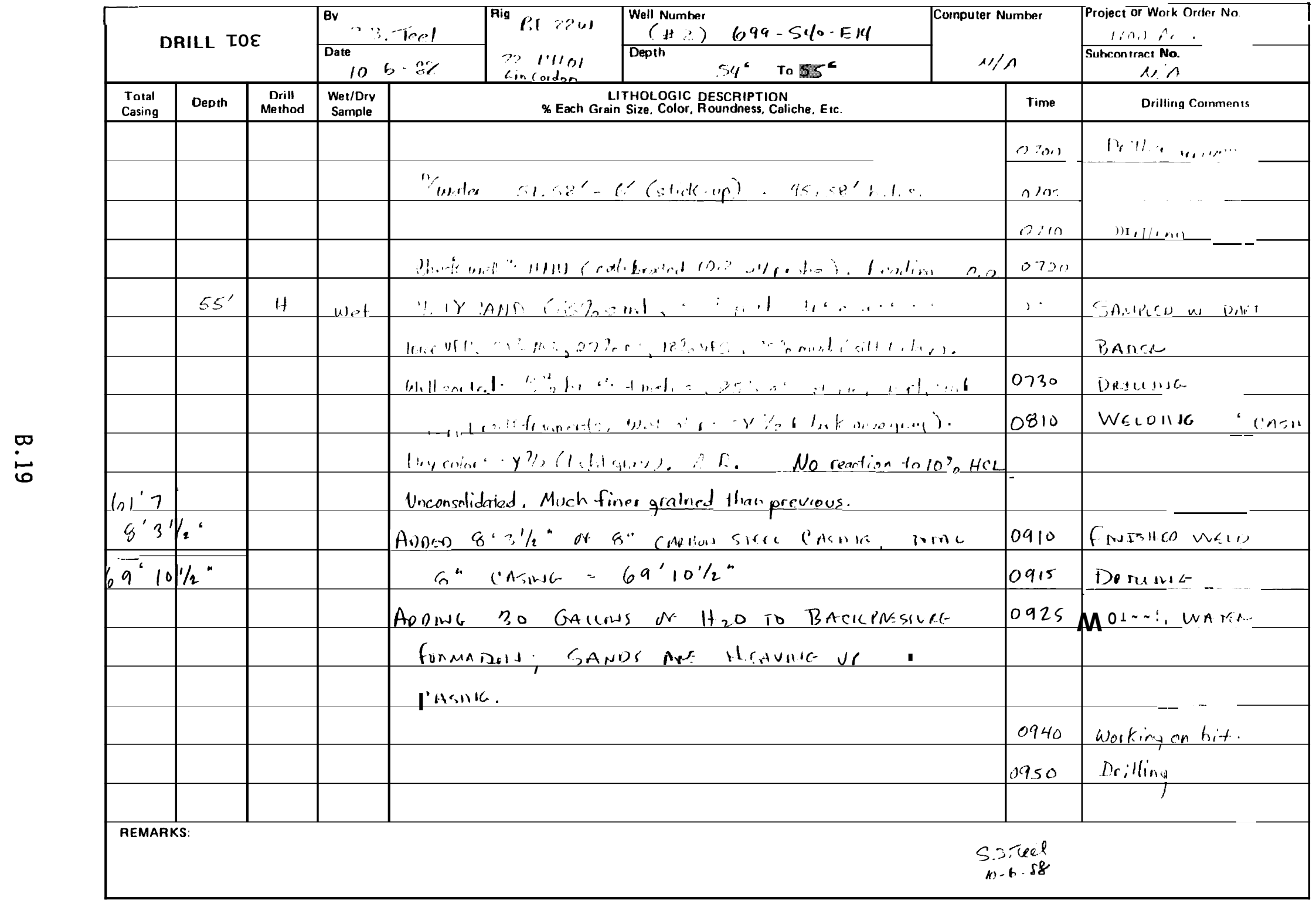

D - Drive Barrel H. Hard Tool L- Large M-Medium S-Small VC-Very Coarse $\quad$ C - Coarse F- Fine VF - Very Fine _- Standing Water 
$\therefore+13$

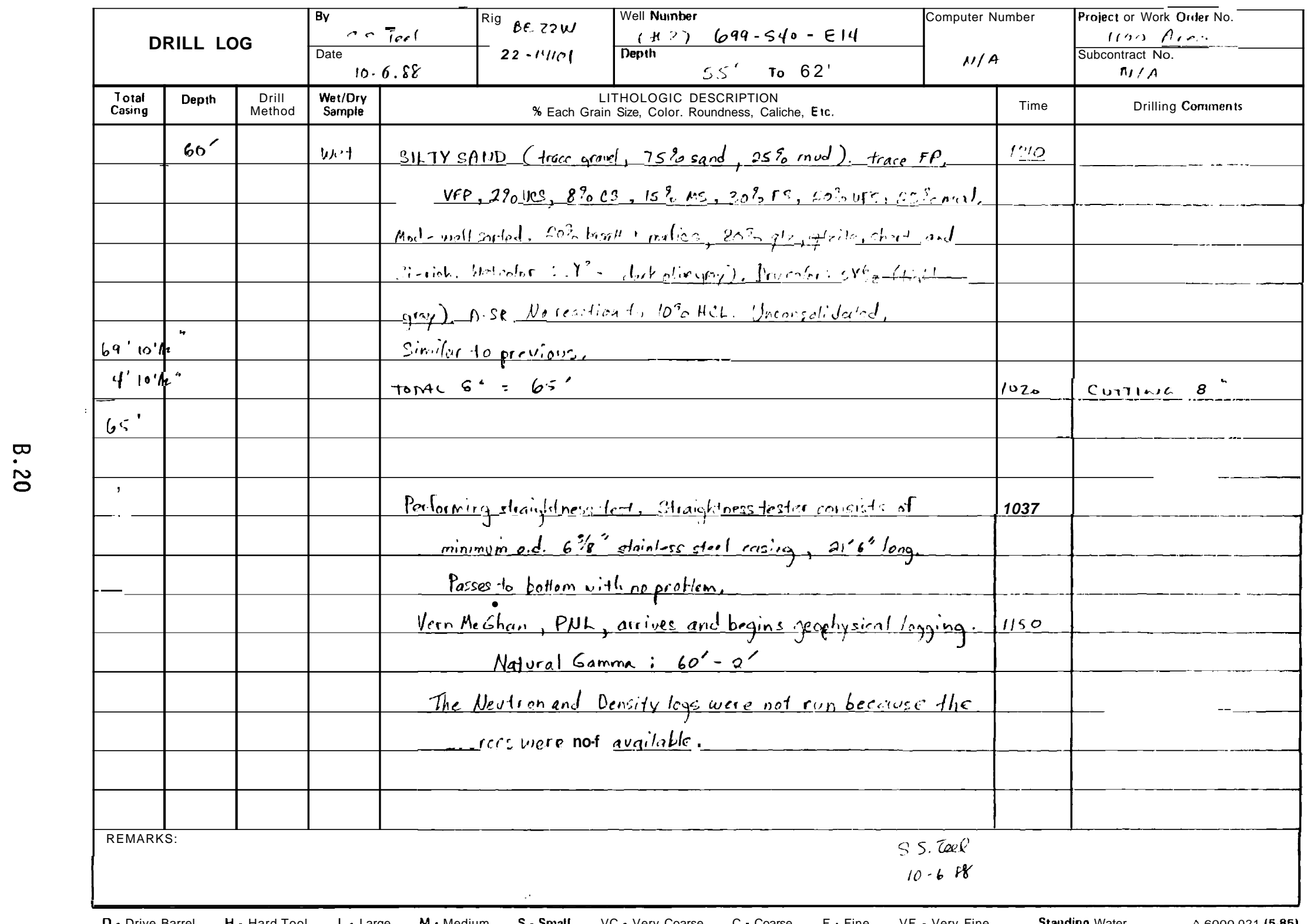

D. Drive Barrel $\quad$ H. Hard Tool L. Large $\quad$ M. Medium S. Small $\quad$ VC. Very Coarse $\quad$ C. Coarse F. Fine VF - Very Fine ___ Standing Water 


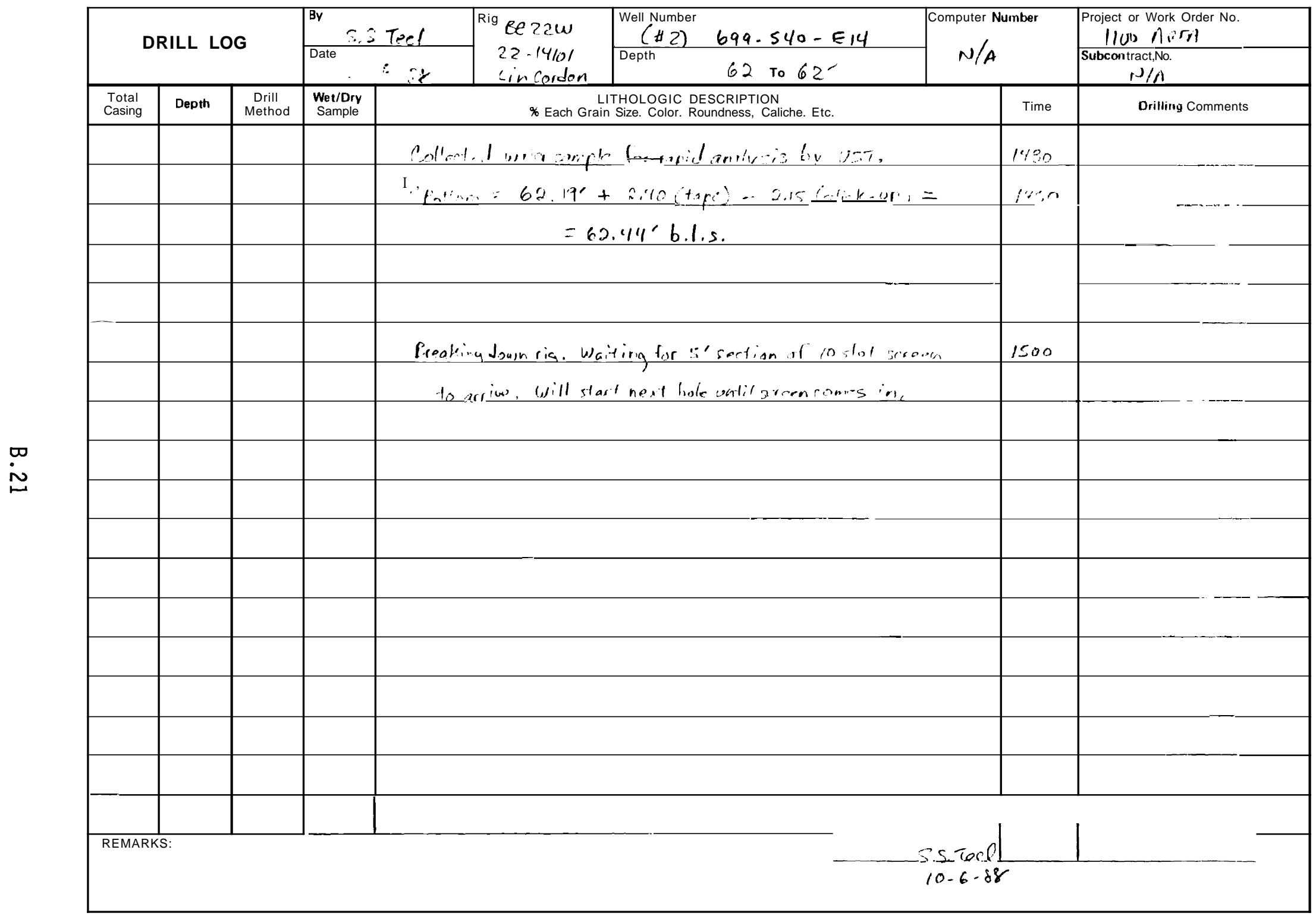

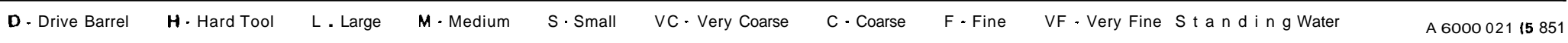




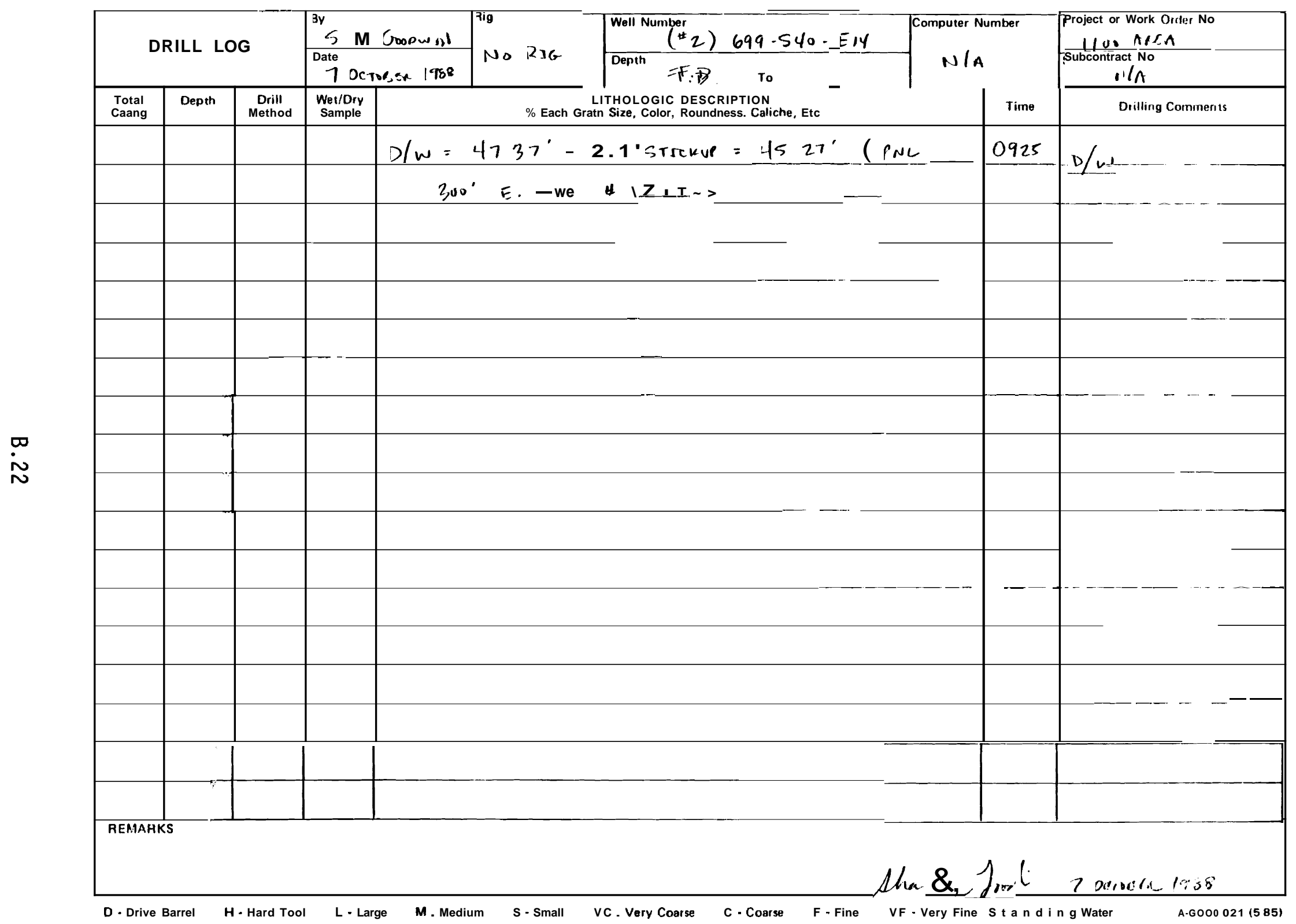


I or 1

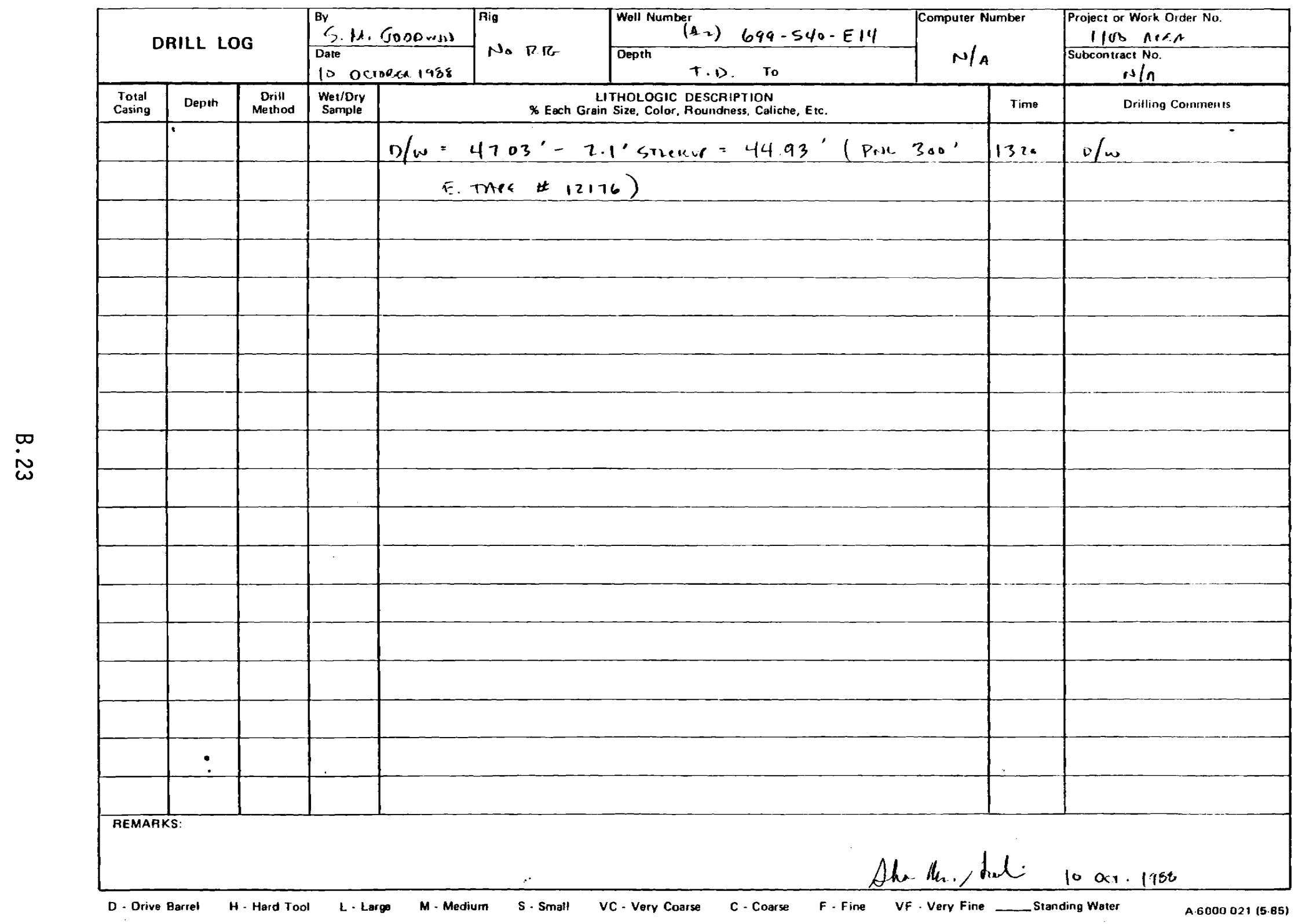


1001

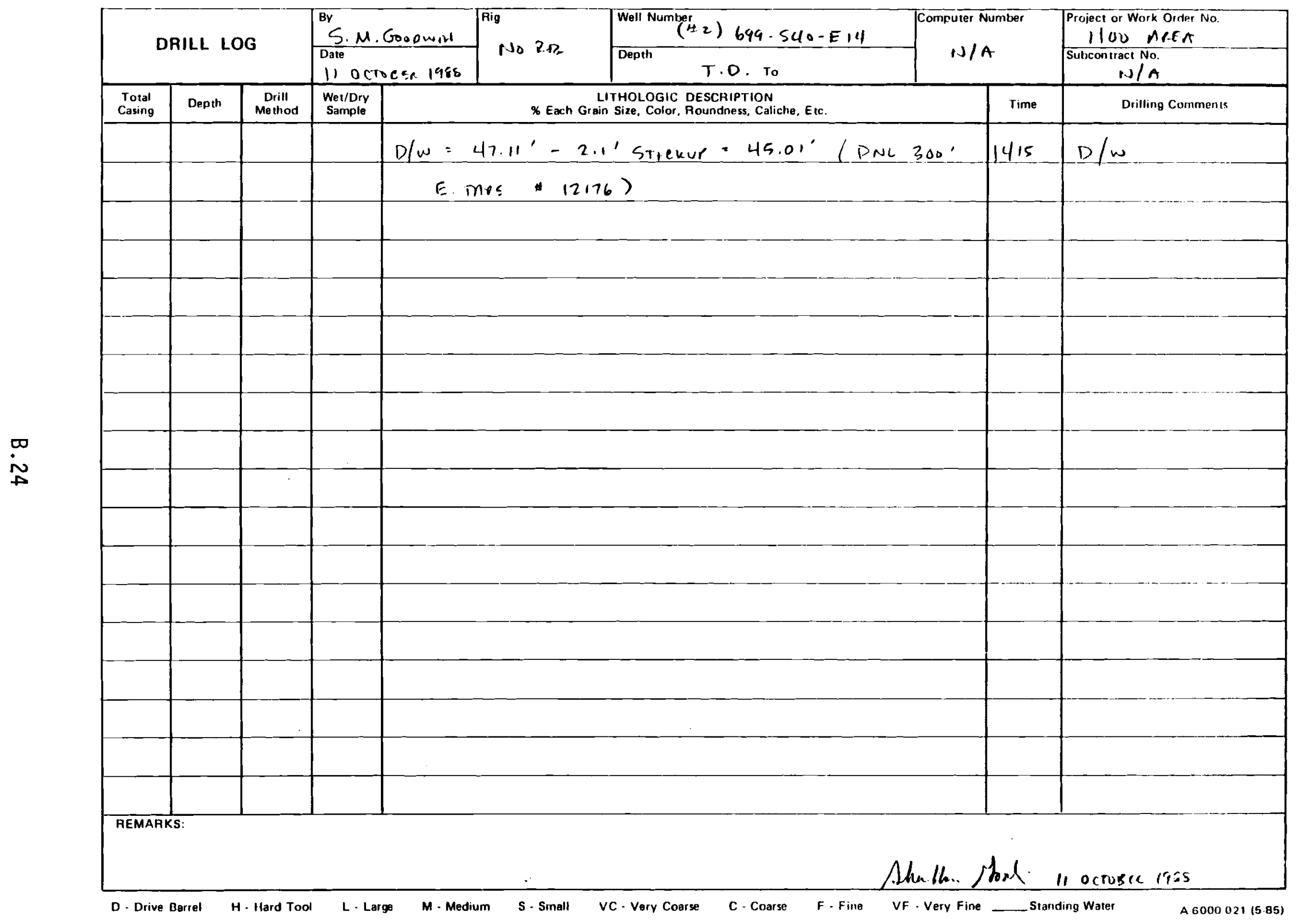


lof 1

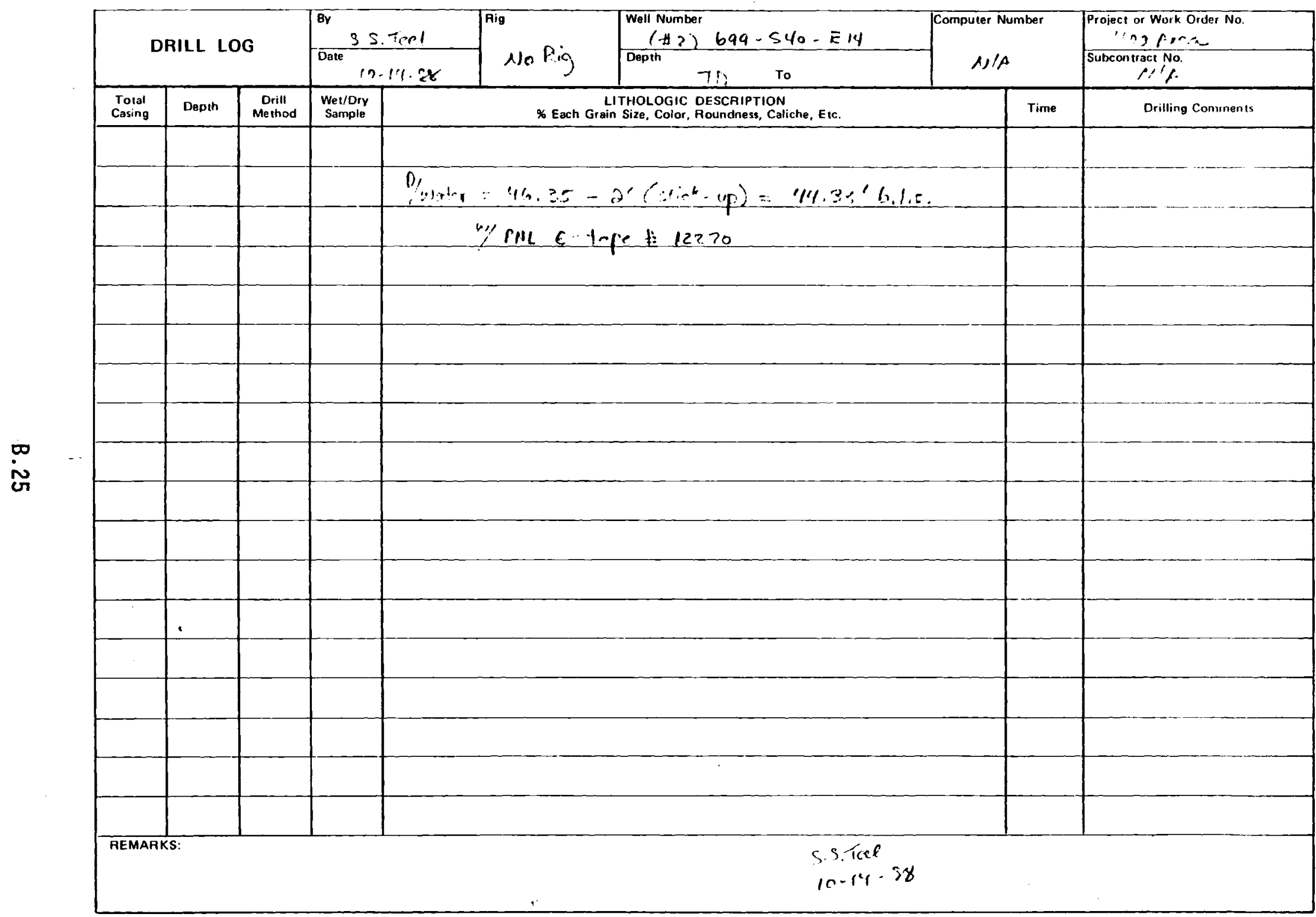

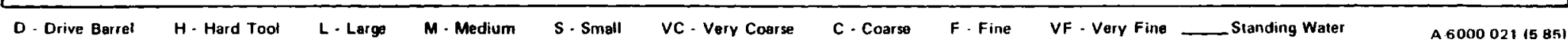


1.62

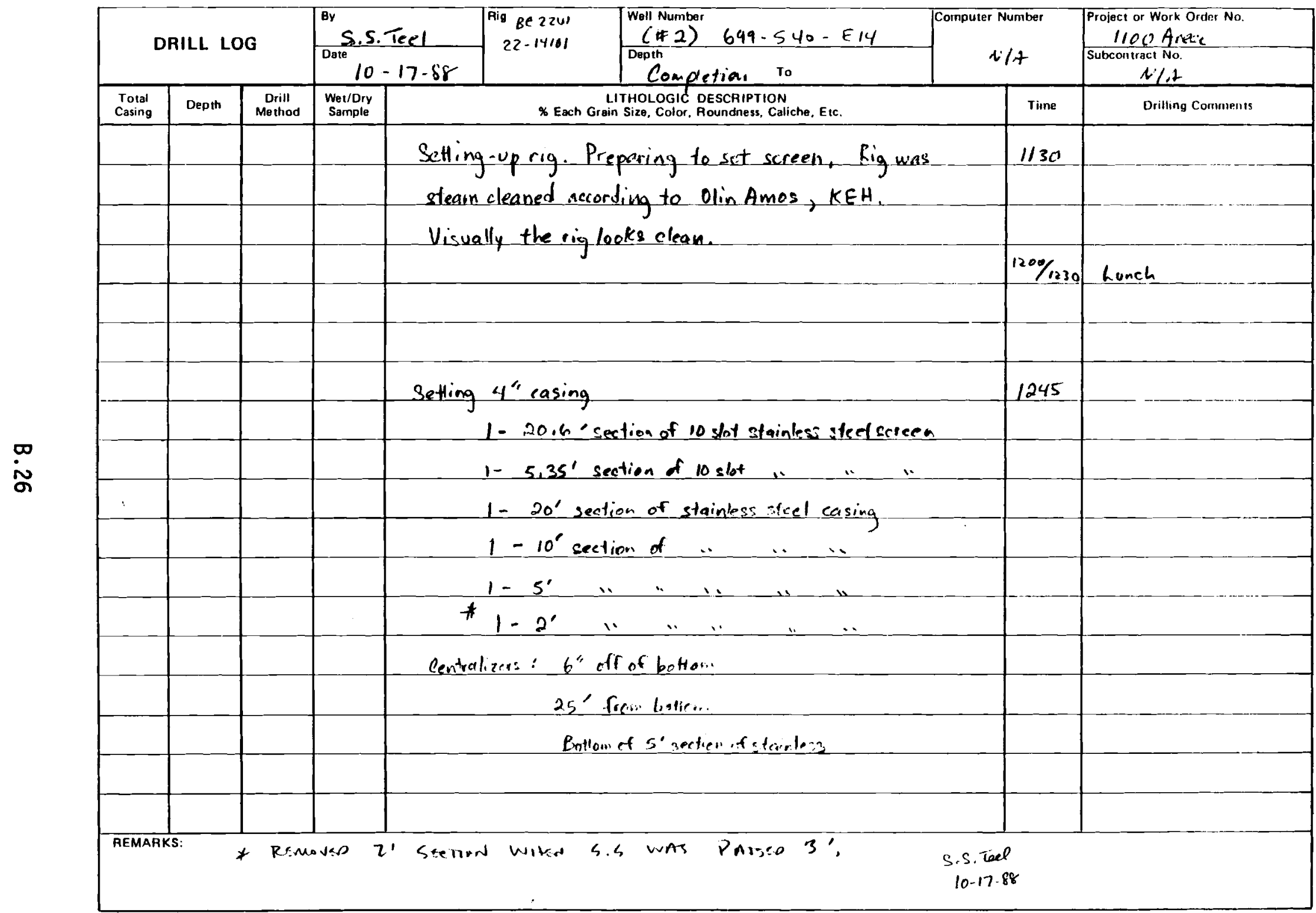

D- Drive Barrel H- Hard Tool L-Large M-Medium S-Small VC-Very Coarse C - Coarse F- Fine VF - Very Fine _- Standing Water 
2 of 2

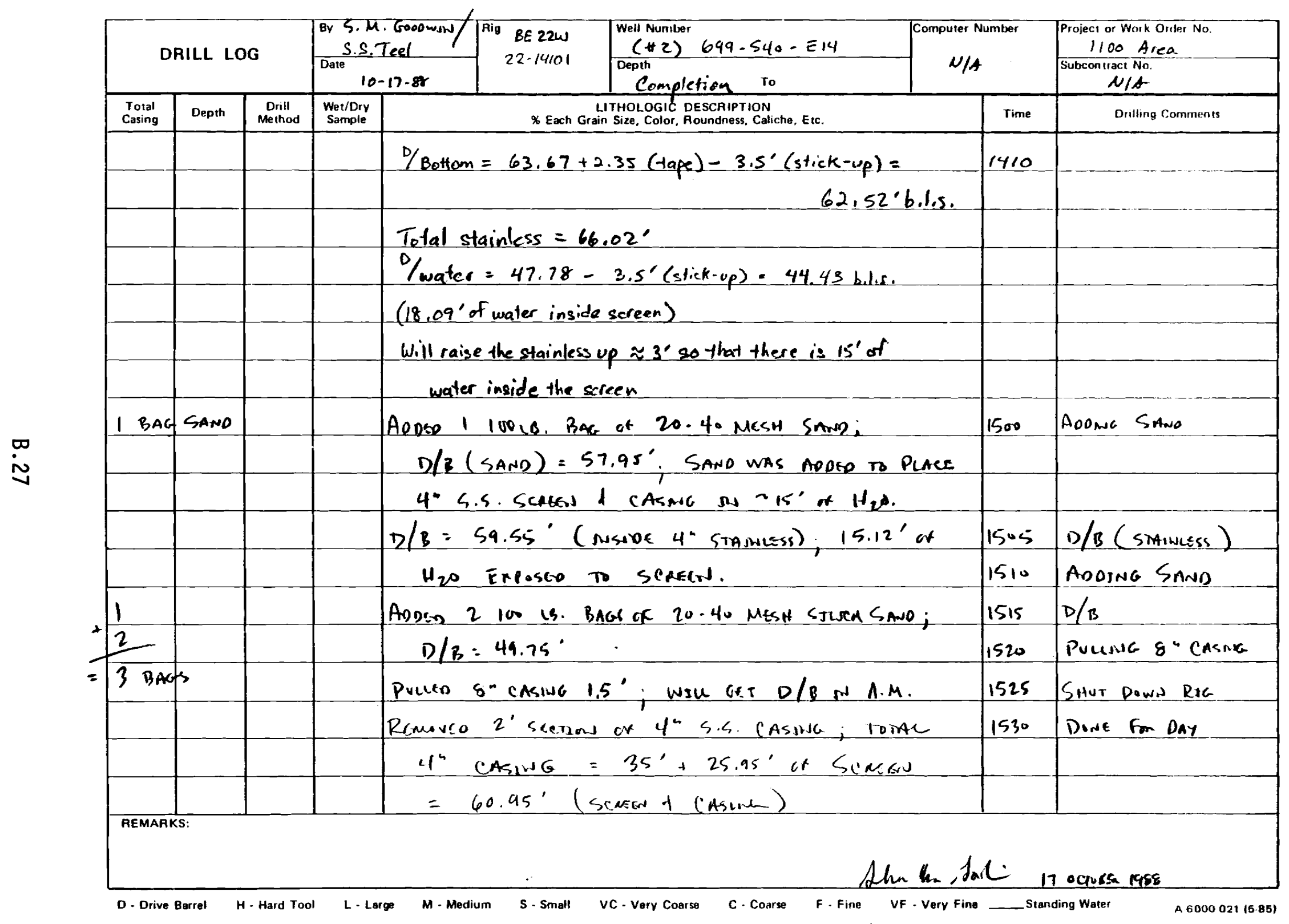




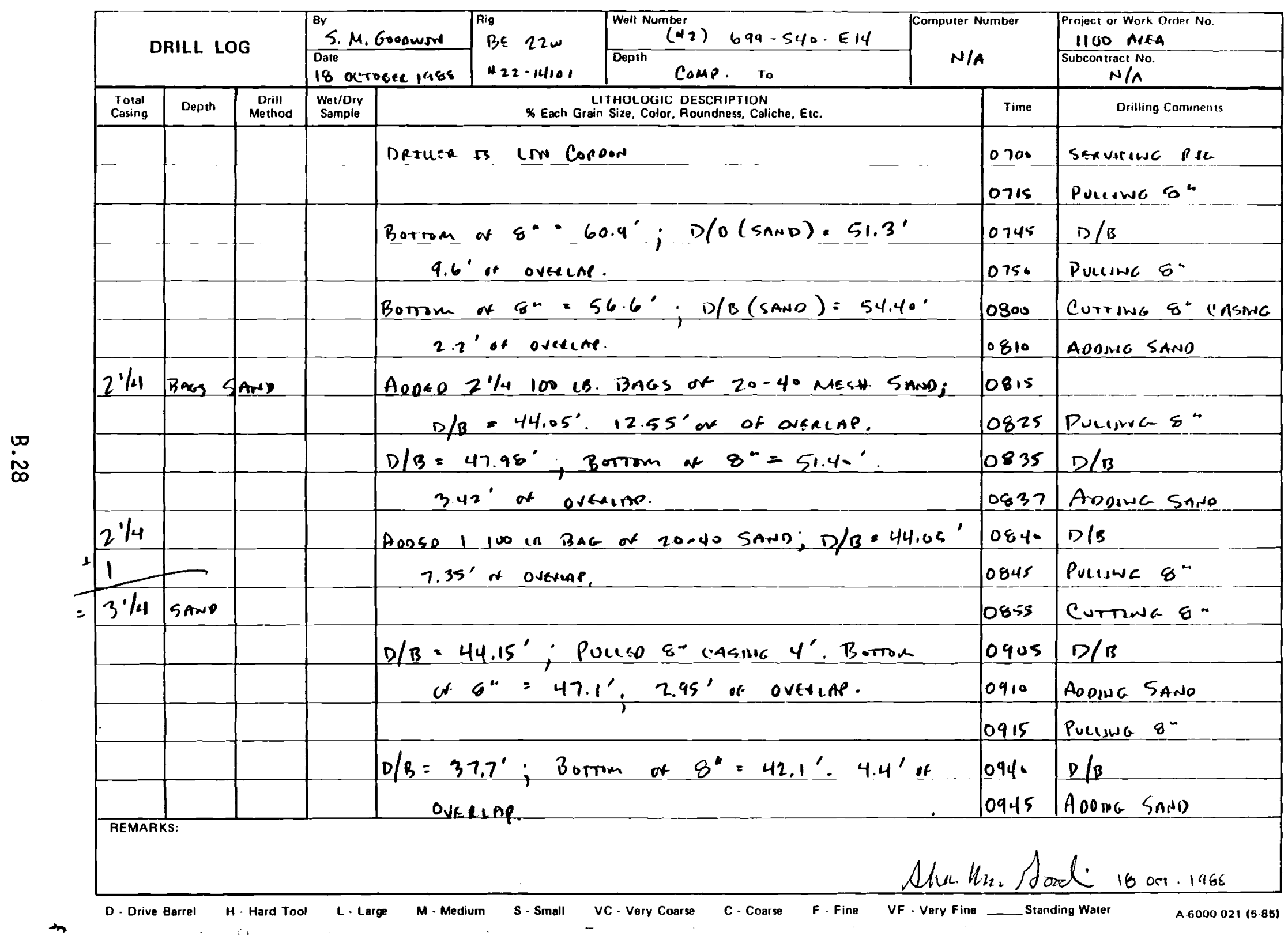




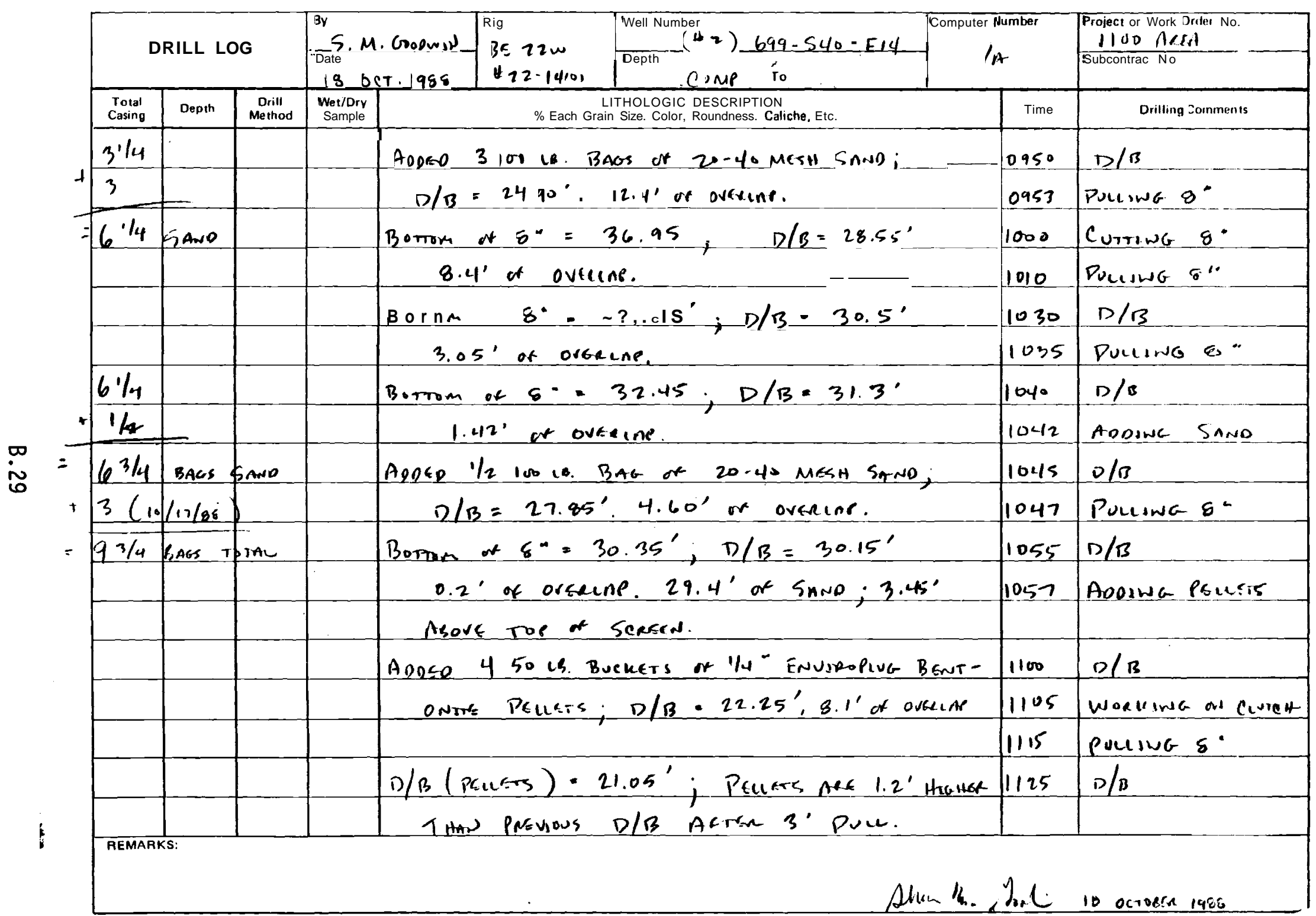

D. Drive Barrel H. Hard Tool L. Large M. Medium S. Small VC. Vary Coarse C.Coarse F. Fine VF. Very Fine Standing Water Agarn 
3 of 4

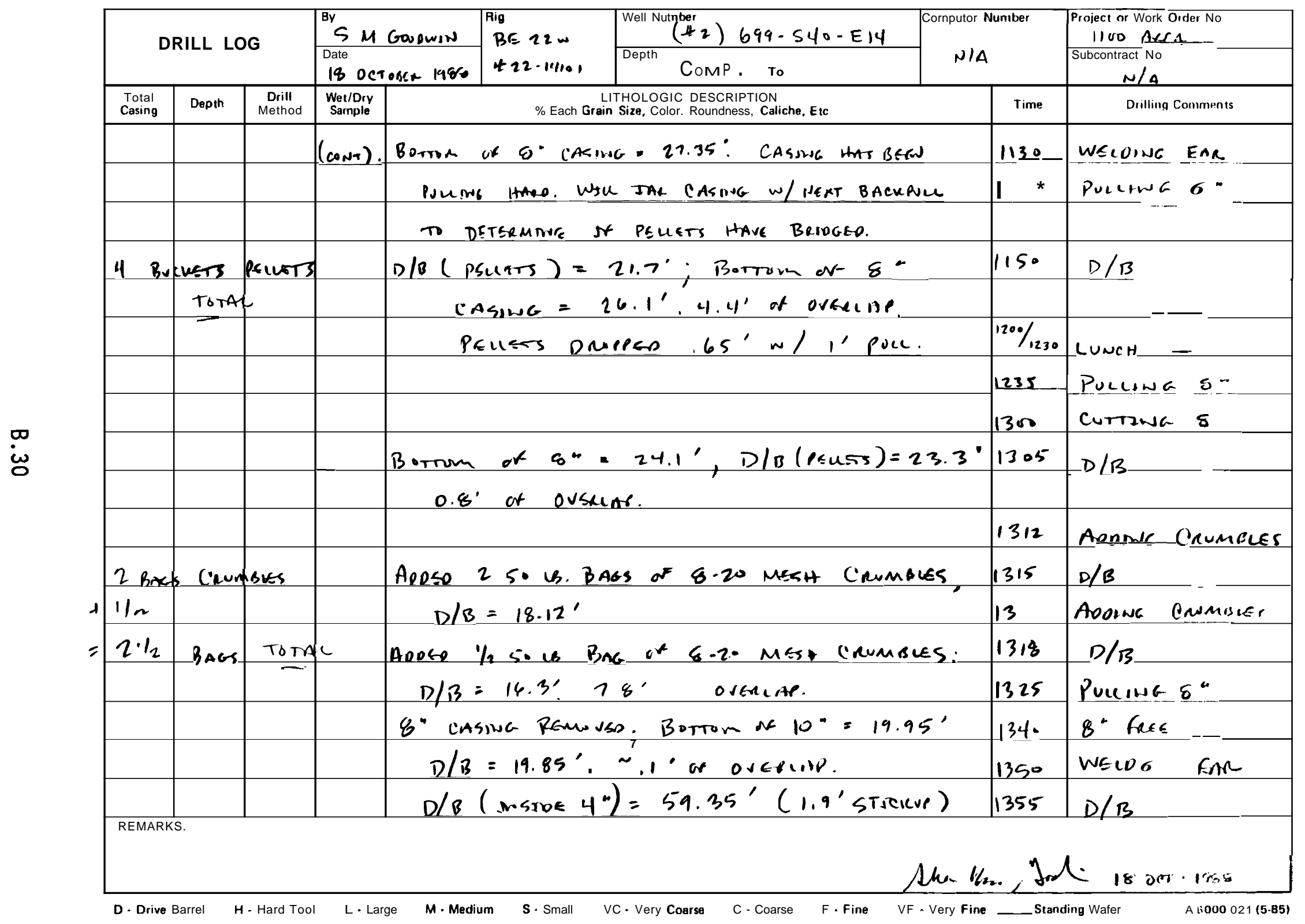


4 of 4

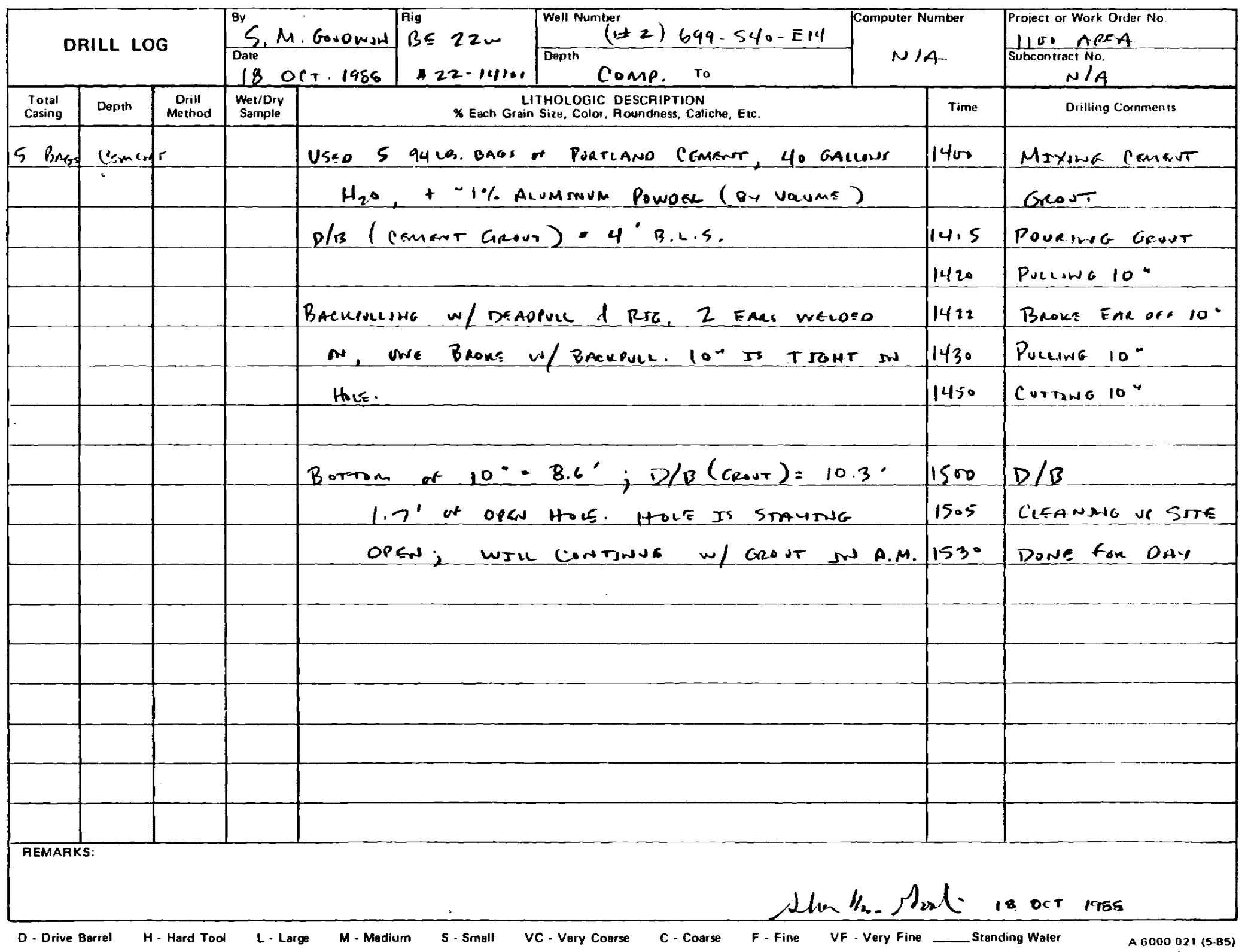




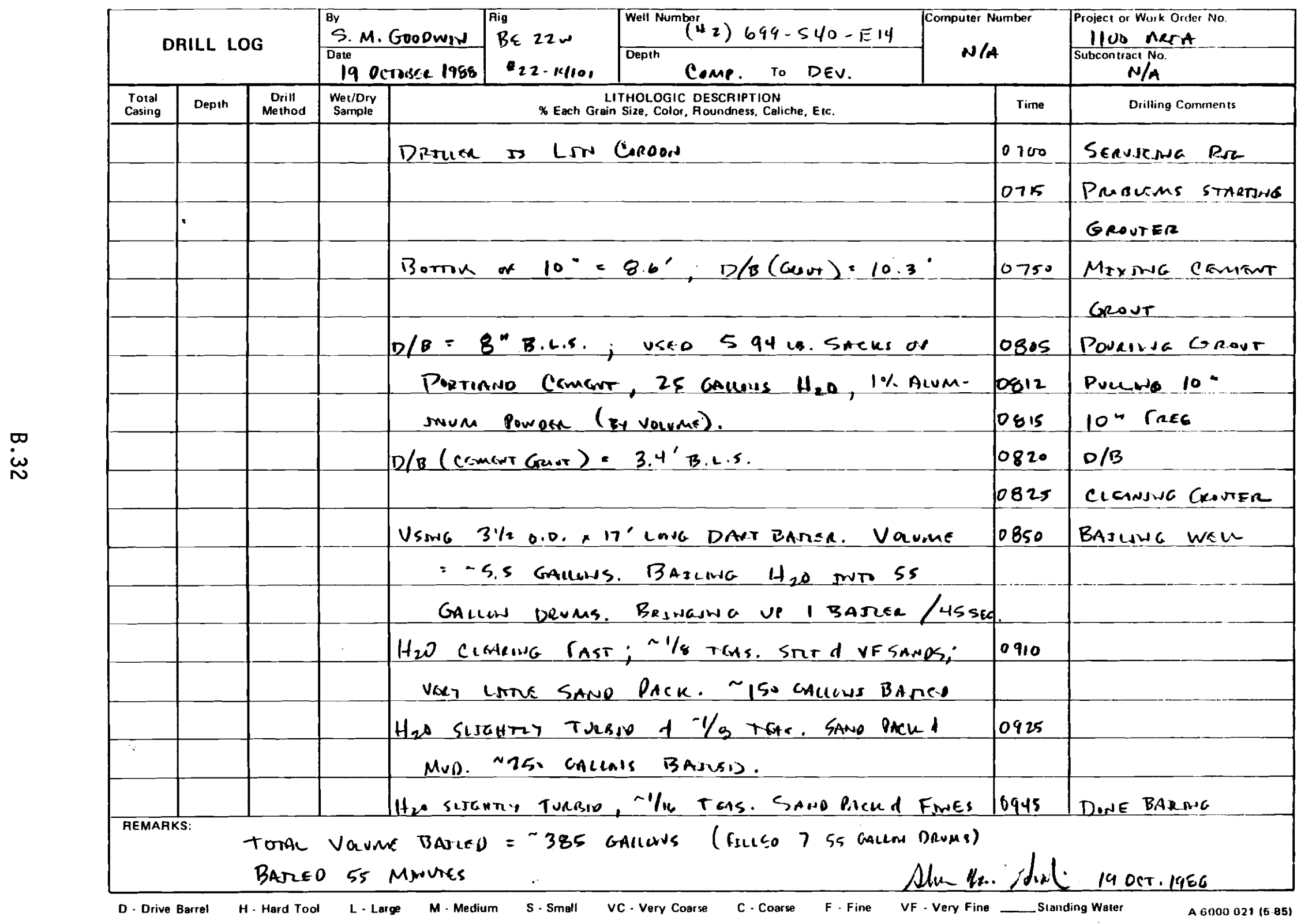


1 or 1

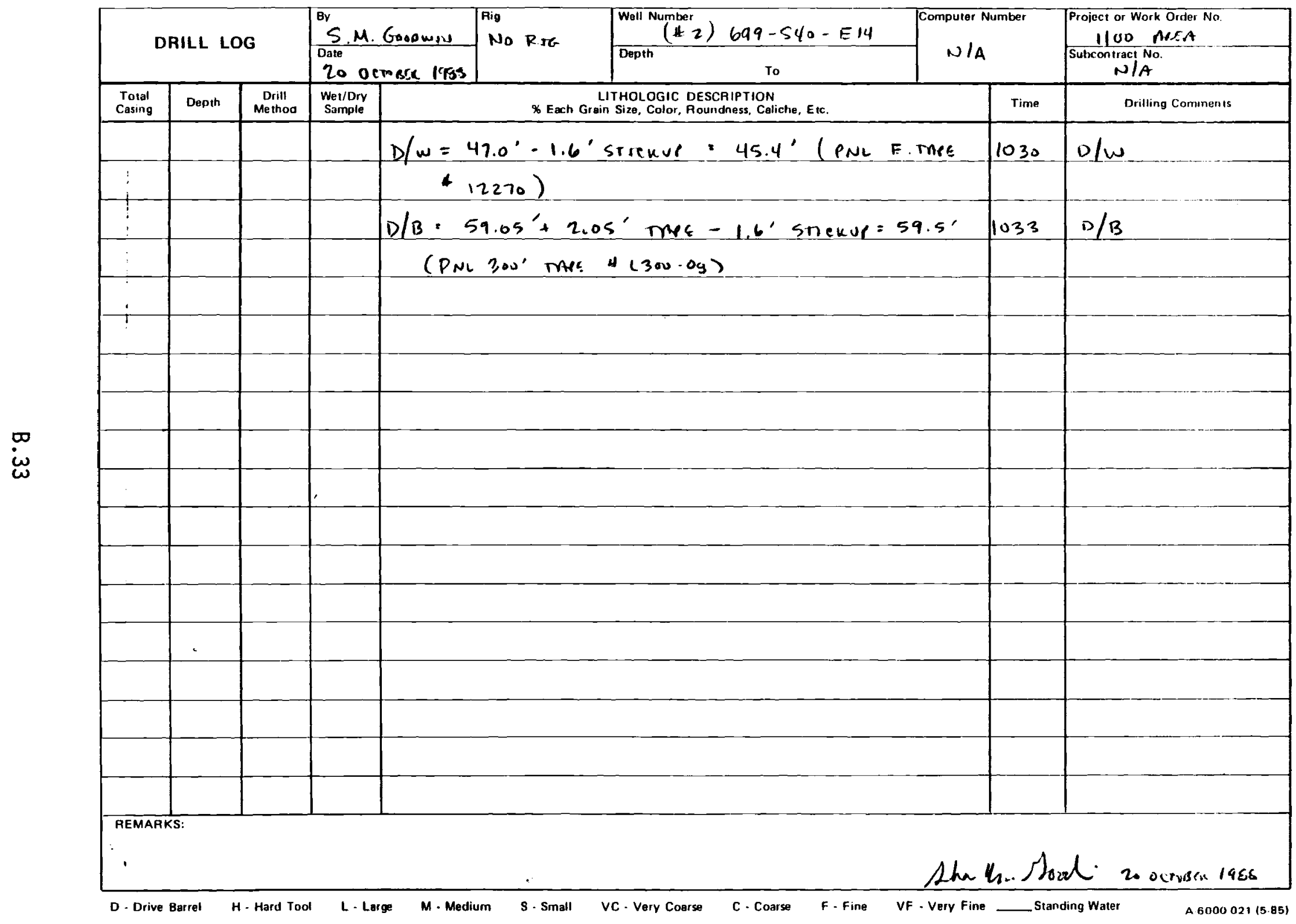


101

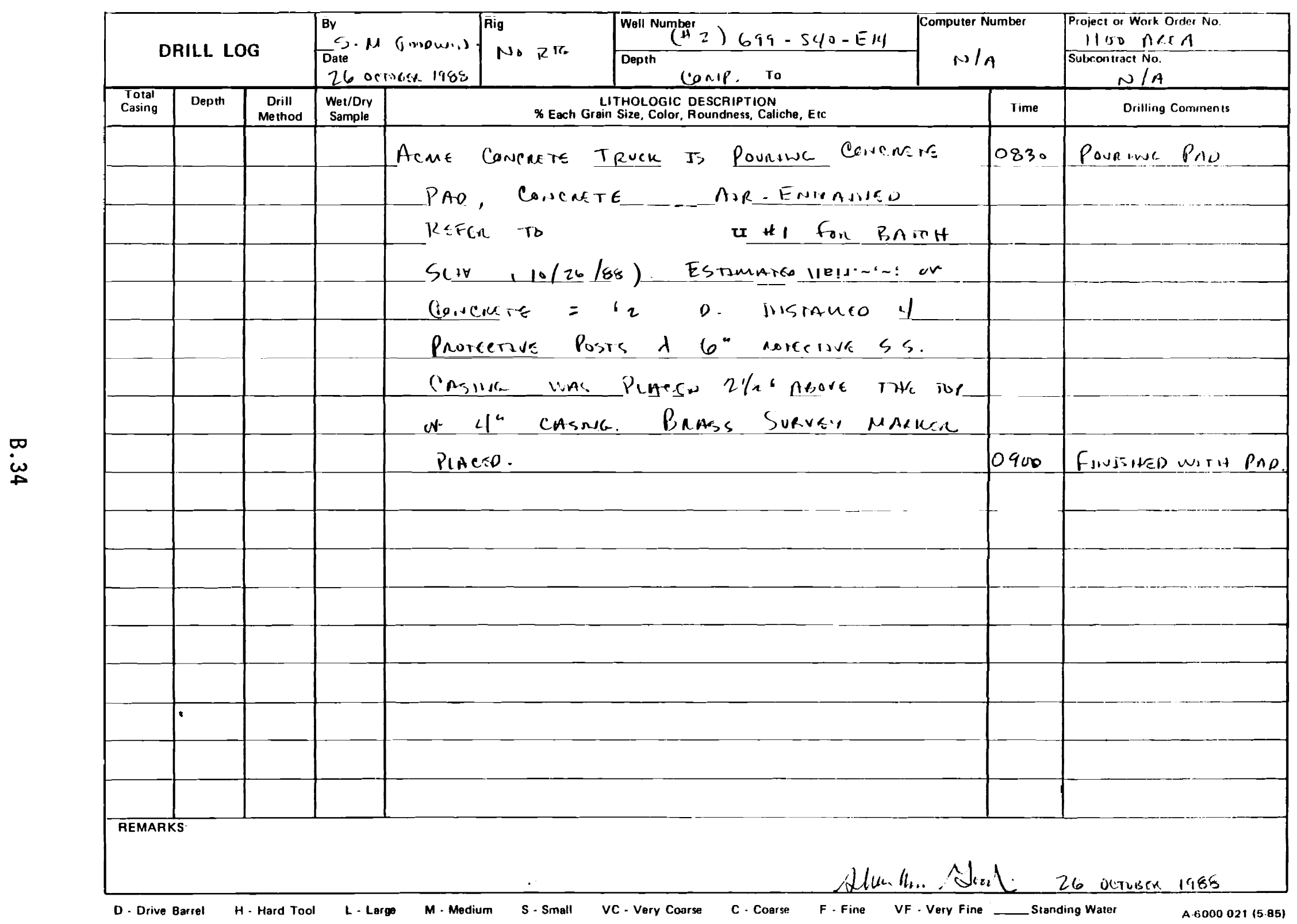




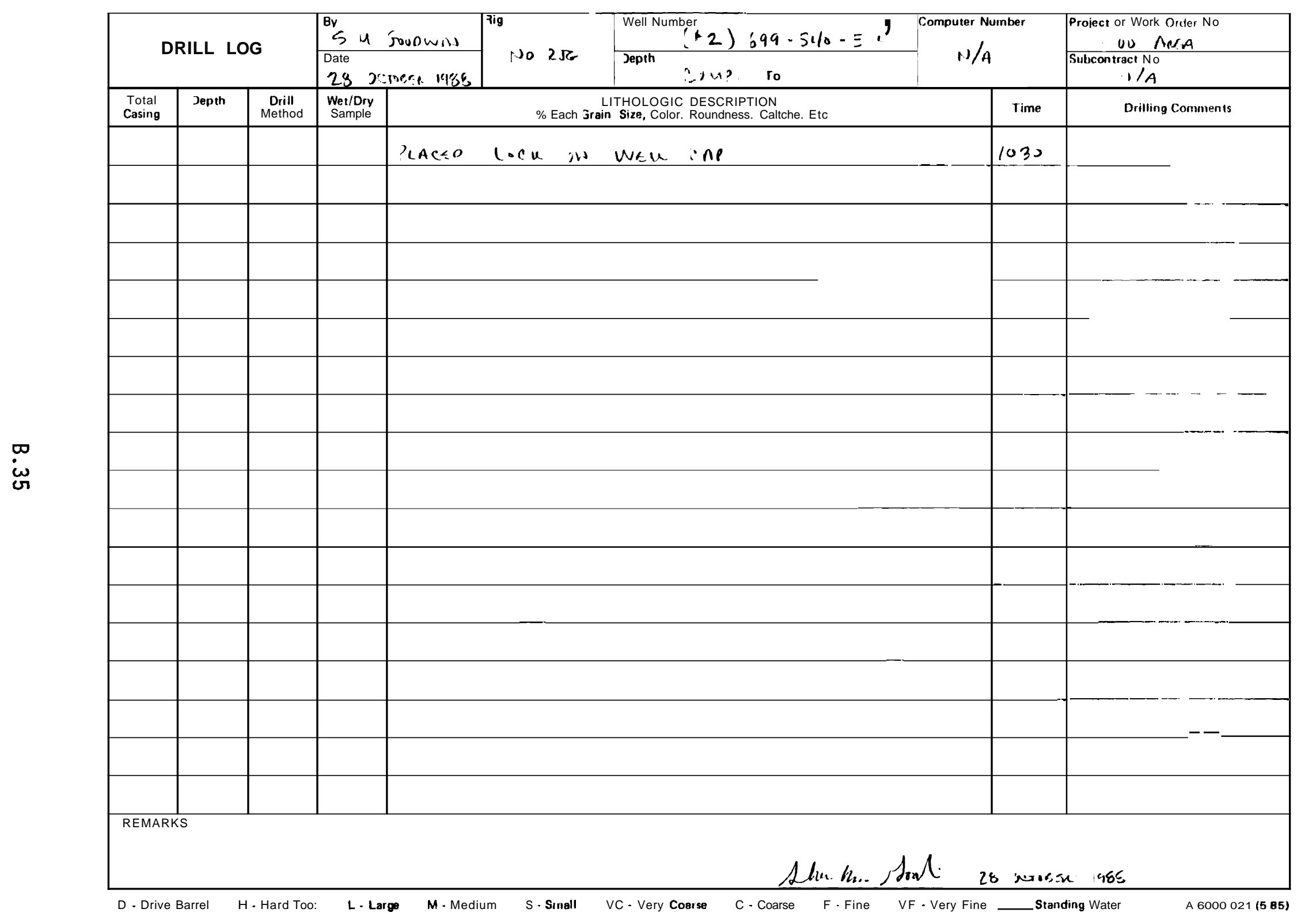




\begin{tabular}{|c|c|c|c|c|c|c|}
\hline \multirow{2}{*}{\multicolumn{3}{|c|}{ DRILL LOG }} & \multirow{2}{*}{ 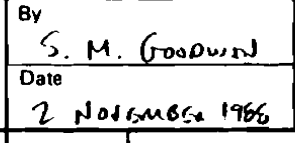 } & \multirow{2}{*}{\multicolumn{2}{|c|}{ 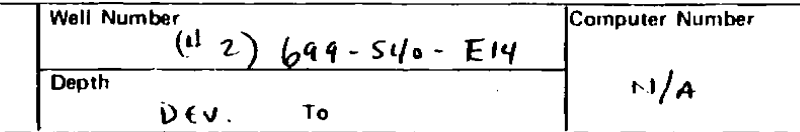 }} & \multirow{3}{*}{$\begin{array}{l}\text { Project or Work Order No. } \\
\text { I1 uo Aren } \\
\text { Subcontract No. } \\
\text { N/A } \\
\text { Drilling Comments }\end{array}$} \\
\hline & & & & & & \\
\hline $\begin{array}{c}\text { Total } \\
\text { Casing }\end{array}$ & Depth & $\begin{array}{c}\text { Drill } \\
\text { Method }\end{array}$ & $\begin{array}{l}\text { Wet/ory } \\
\text { Sample }\end{array}$ & $\begin{array}{l}\text { LITHOLOGIC DESCAIPTION } \\
\text { \% Each Grain Size, Color, Roundness, Caliche, Etc. }\end{array}$ & Time & \\
\hline & & & & USING A 1.5 H.P. GRUNDFOS SUBMSASHOVE & 09.5 & Doracizea renos \\
\hline & & & & PUMP to DEVELP WEU BY OVERPUMPSOSG. & & 5230 canors \\
\hline & & & & Pume cocuma is Ser Ar Bortom of $4^{\circ}$ & & \\
\hline & & & & Sivmune ts $\sim 1.5^{\prime}$ from Zoriom or Pump & & \\
\hline & & & & Cocuma. $H_{1} \mathrm{O}$ is Brone Pumpeo wor $A$ & & \\
\hline & & & & WATER TRUEV to PlEVENT RECHARGE of & & \\
\hline & & & & MUNJeIPAC WATER UGUS W/DEVGloPMirs & & \\
\hline & & & & 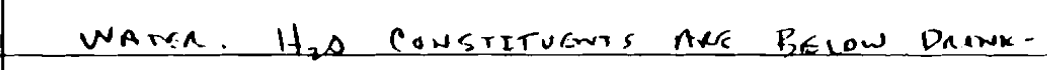 & & \\
\hline & & & & NWE H,O STANDAKOS. & & \\
\hline & & & & $D / w=50.8^{\circ}$ (PNL E. Mners $412021^{2}$ Fron & 0920 & $D / w$ \\
\hline & & & & IDP of TREMIE PIPC) & 0925 & Pume orl \\
\hline & & & & Flow rates Aere freom flow MERSR; 10 Gaunus & 0926 & \\
\hline & & & & Pumpto / 49.11 ste. $0 / w=50.9^{\prime}$ & 0927 & $D / \omega ; 12.2 \mathrm{GAL} / \mathrm{mns}$ \\
\hline & & & & H 20 SAMPUE $=0.87$ NTV; Clsar 1 CLCAN & 0929 & \\
\hline & & & & Veav line seomeroves & & \\
\hline & & & & $D / w=50.9^{\prime}$ & 0930 & $D / w$ \\
\hline & & & & 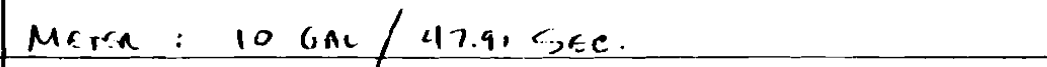 & 10931 & $12.5 \mathrm{cmc} / \mathrm{Mm}$ \\
\hline & & & & 1t 20 SAMPLE $=0.57 \mathrm{NTU}$ & 10934 & \\
\hline AEMAF & & & & I. & $n_{2} \ldots, 2$ & $11 / 2 / 88$ \\
\hline
\end{tabular}

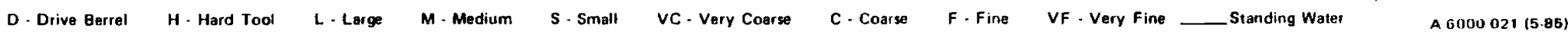




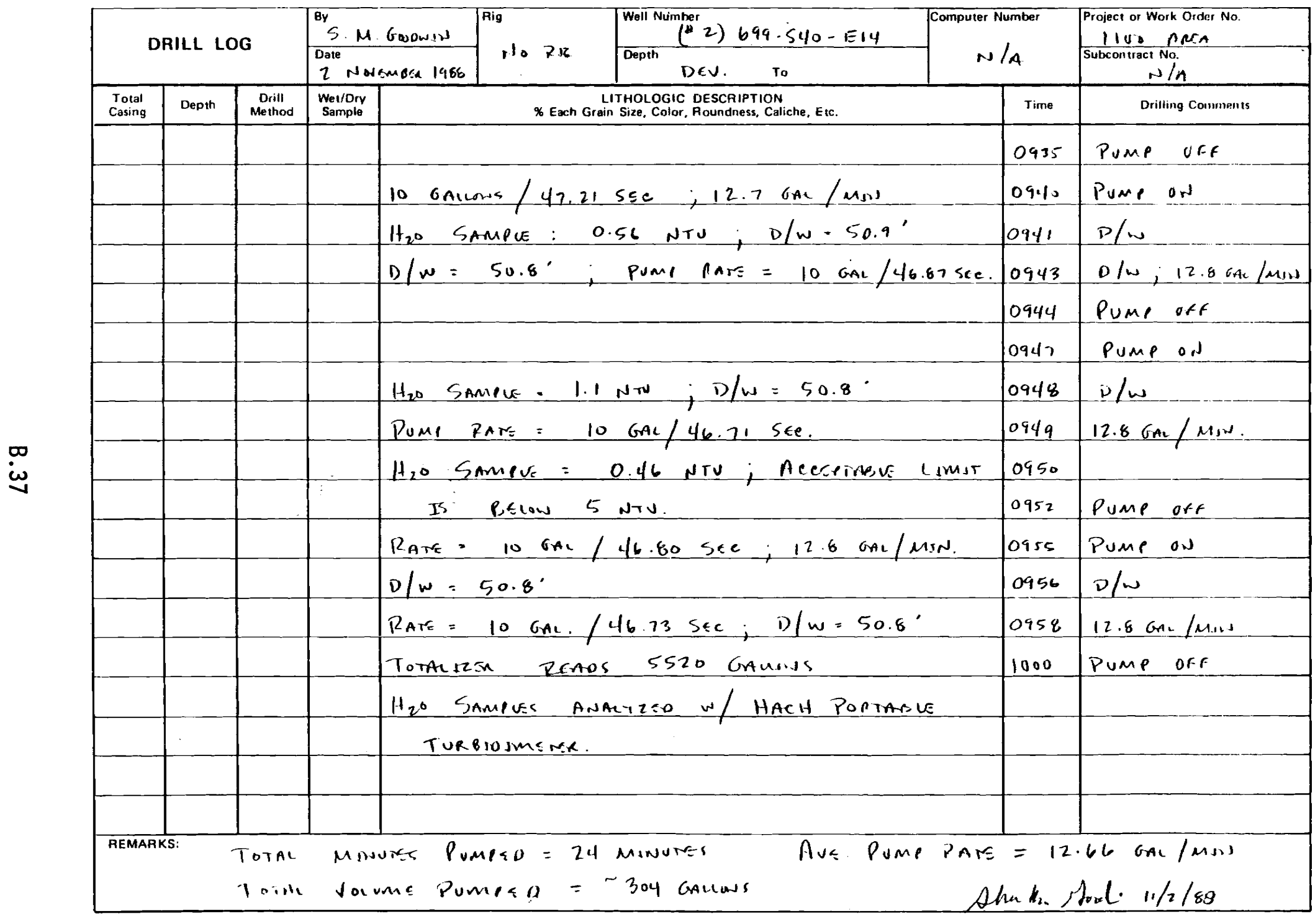

D. Drive Barrel H- Hard Tool L-Large M-Medium S-Small VC-Very Coarse C. Coarse F-Fine VF-Very Fine _ Standing Water A.6000.021 (5.85) 
1082

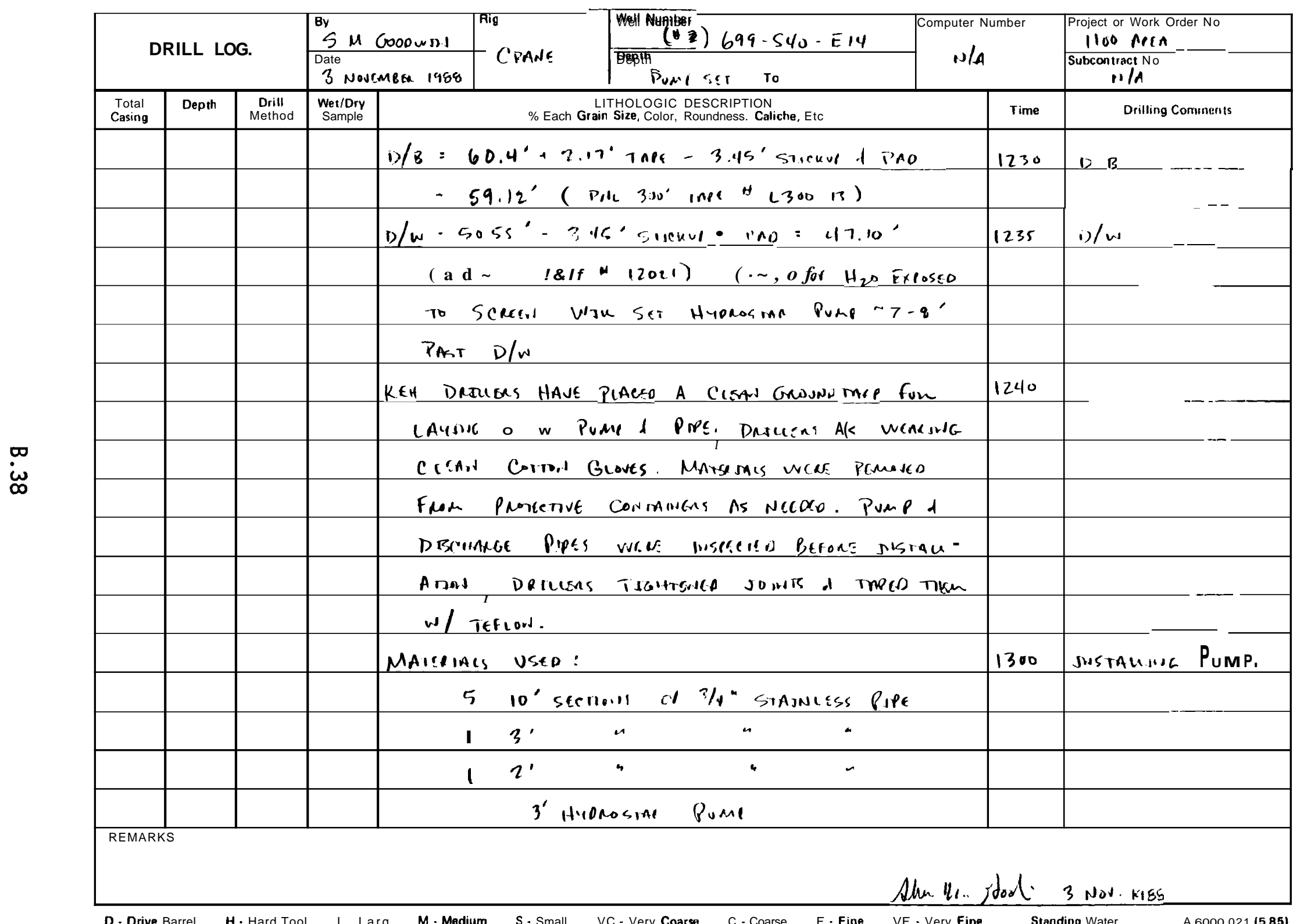


$2+2$

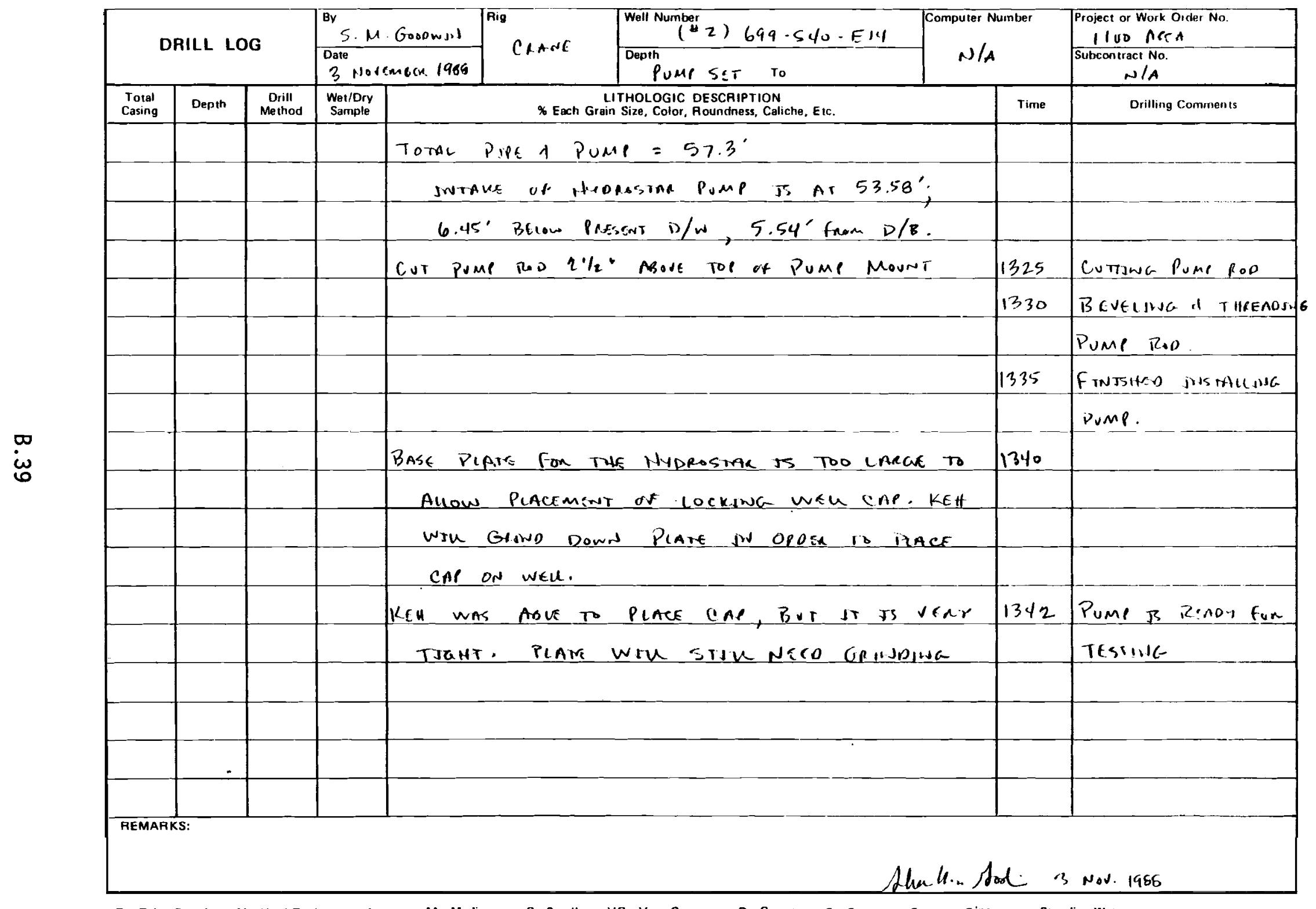

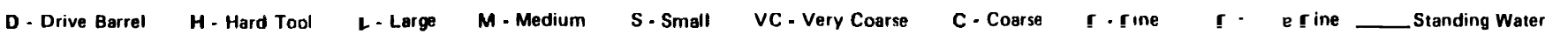

A $6000027(585)$ 


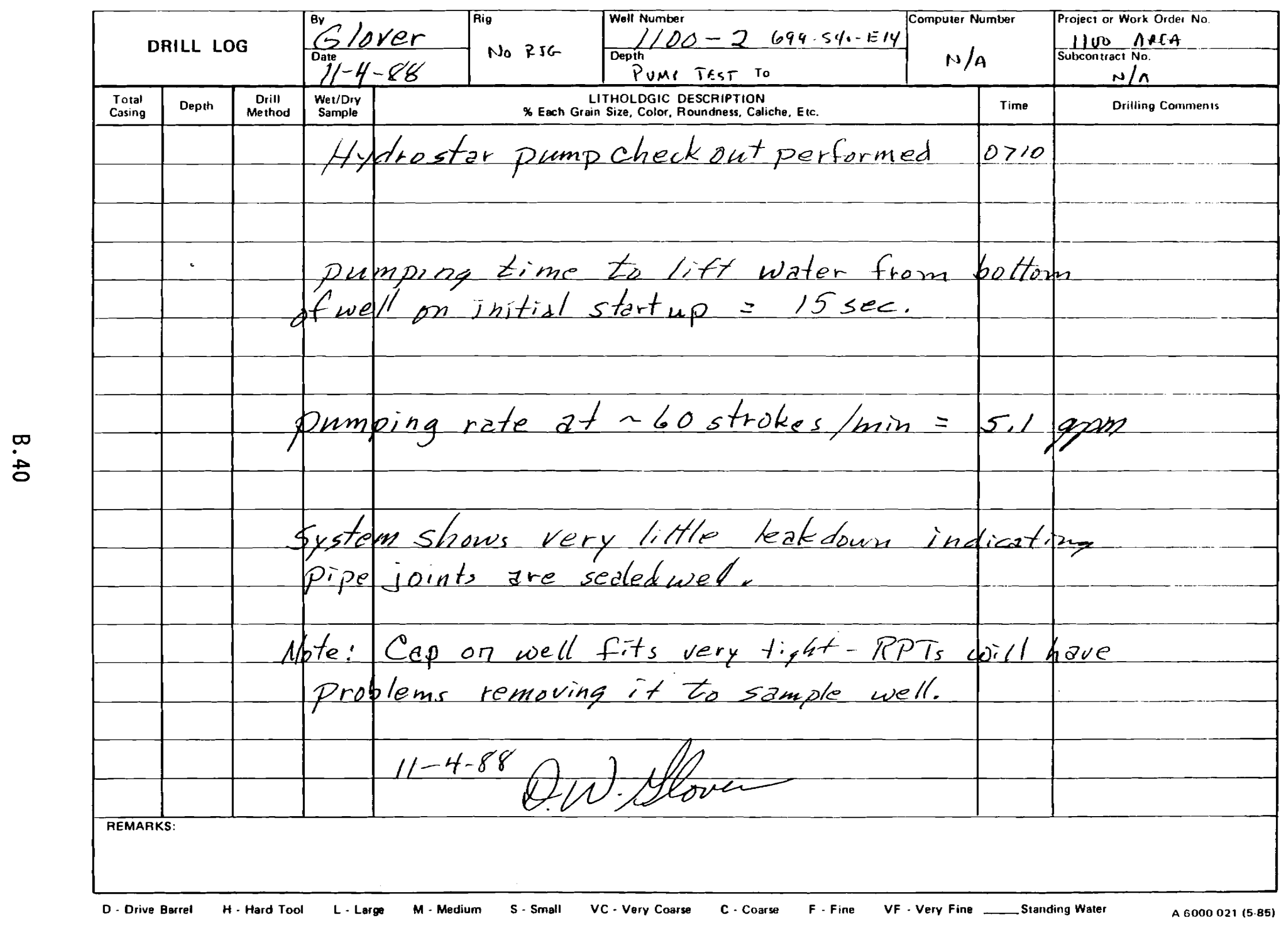




\section{APPENDIX C}

GEOPHYSICAL AND GEOLOGIC LOGS FOR WELL 3 (699-S41-E13B) 


\section{APPENDIX C}

\section{GEOPHYSICAL AND GEOLOGIC LOGS FOR WELL 3 (699-S41-E13B)}

This appendix contains the Well Completion/Inspection Report, as-built diagram, notes from the sampling pump installation, the natural gamma log, and the geologists' logs for well 3 (699-S41-E13B) in the 1100 Area. 
Procedure P-6

Attachment 1

Page 1 of 1

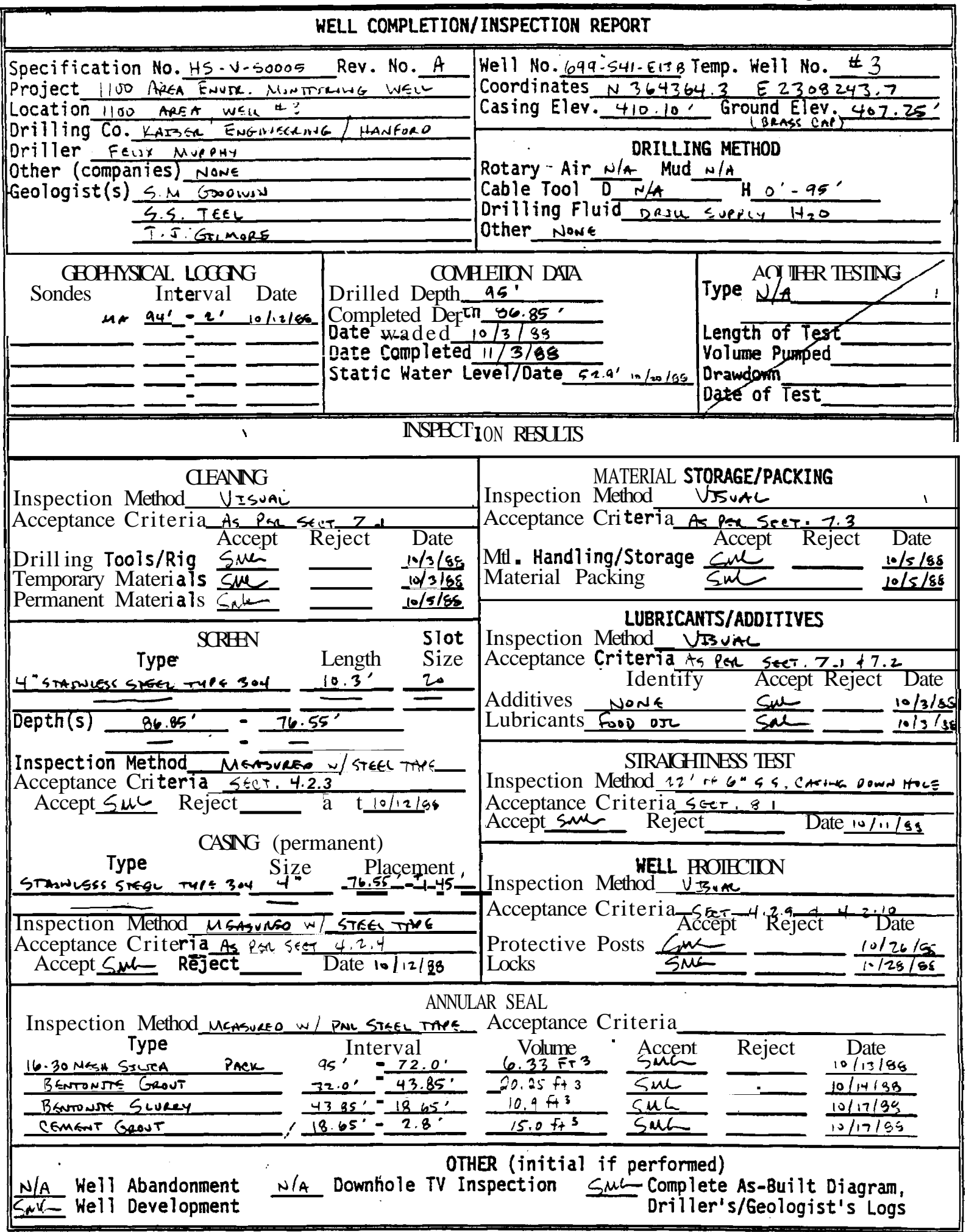

REVIEWED By JiL. Sucthan 11-7-88

For all blanks mark N/A if not applicable. 


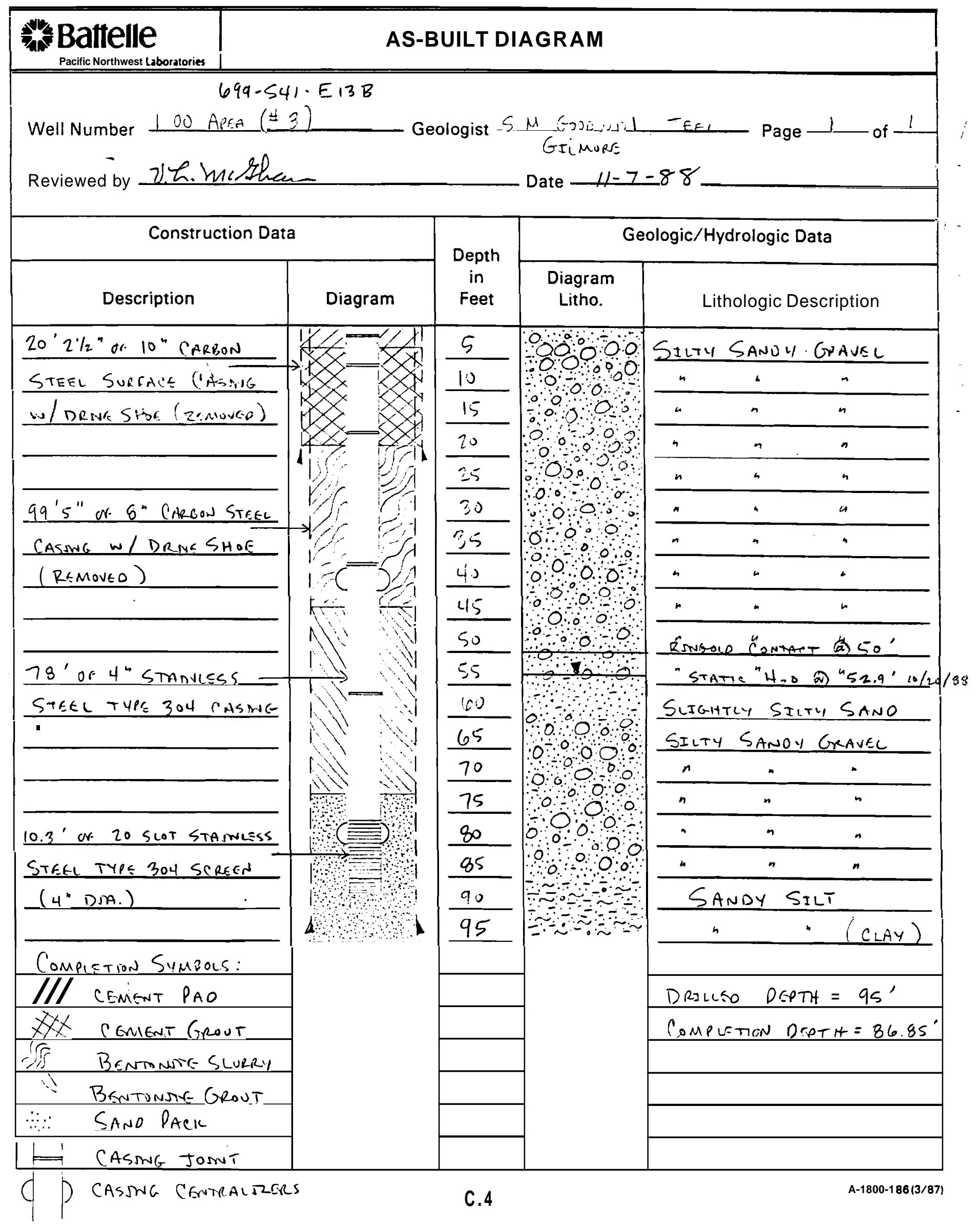


SAMPLING PUMP INSTALLATION IN

GROUND-WATER MONITORING WELLS

Site: 1100 Arst

Monitoring Well Number: (H3) $699-541-E 13 B$

Depth to Water: 54.25

Depth to Bottom: $36.37^{\prime}$ Reported Depth to Bottom: $36.85^{\circ}$

Pump Type: Positive Displacement Piston

Pump Model: Hydrostar HS-8001

Date Instal led: $11 / 3 / 98$

Installed By: KEH : OUN AMOS; LOUJS WATKNNS. LENNY CORDON

Pump Discharge Pipe Description:

$810^{\prime}$ SECTIONS of $3 / 4$ " STANeSS STEEL DISIHARCE PJE

$13^{\prime}$ SECTION ON $3 / 4^{2}$ STANULESS STEEL DTSCHTRCE PJPS

$12^{\prime}$ SECTIOW of $3 / 4$ ' STAJWLSSS STEEL $C$ PNE

$2^{\prime}$ ' $3^{\prime}$ SECTUWS ARE BSTWEEN $10^{\prime}$ ' $15^{\prime}$ BSLOW. BASE PLATE

Additional Comments:

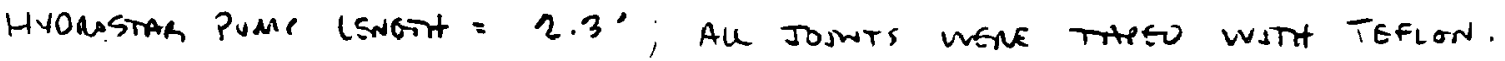

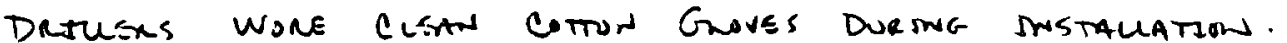

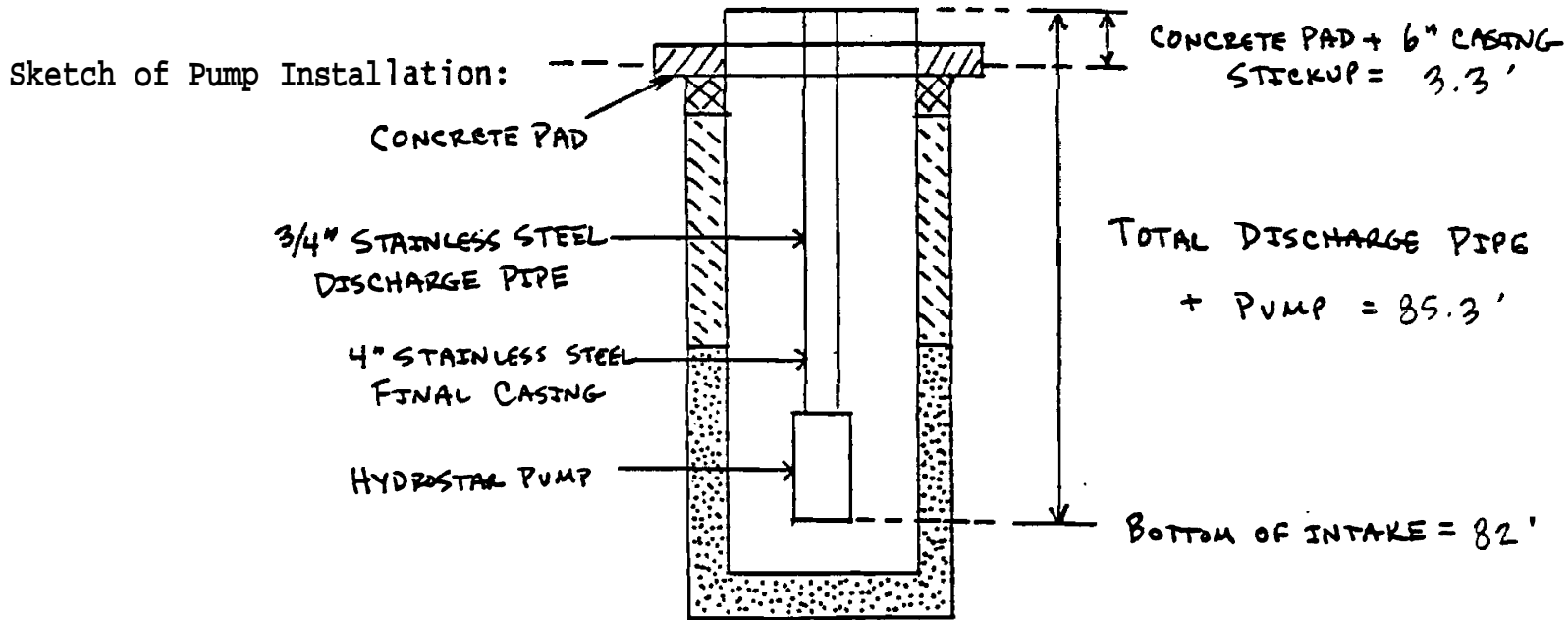

Completed By: Ahkna Hal:

Date: $11 / 3 / 35$

C. 5 


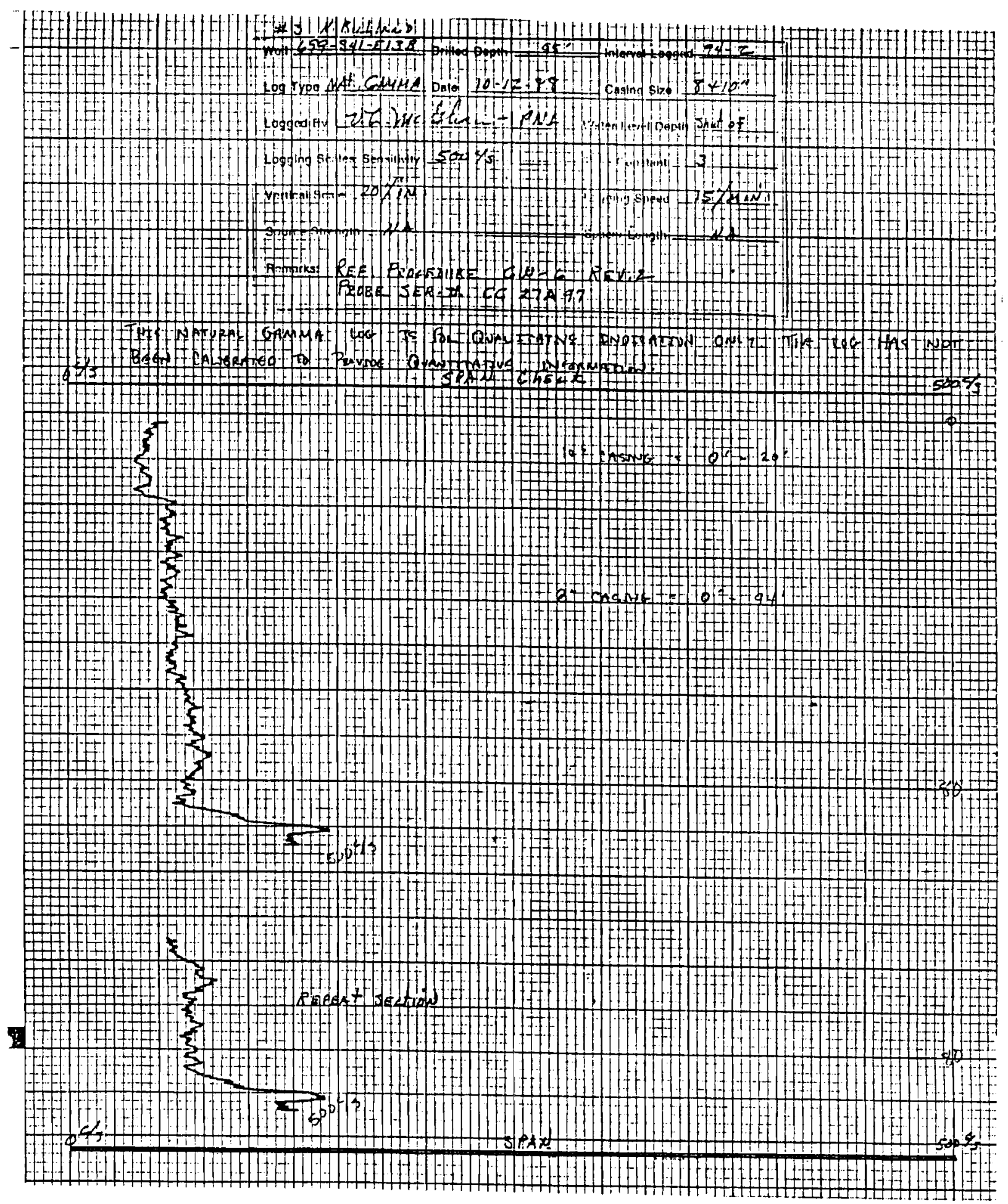


1 of 2

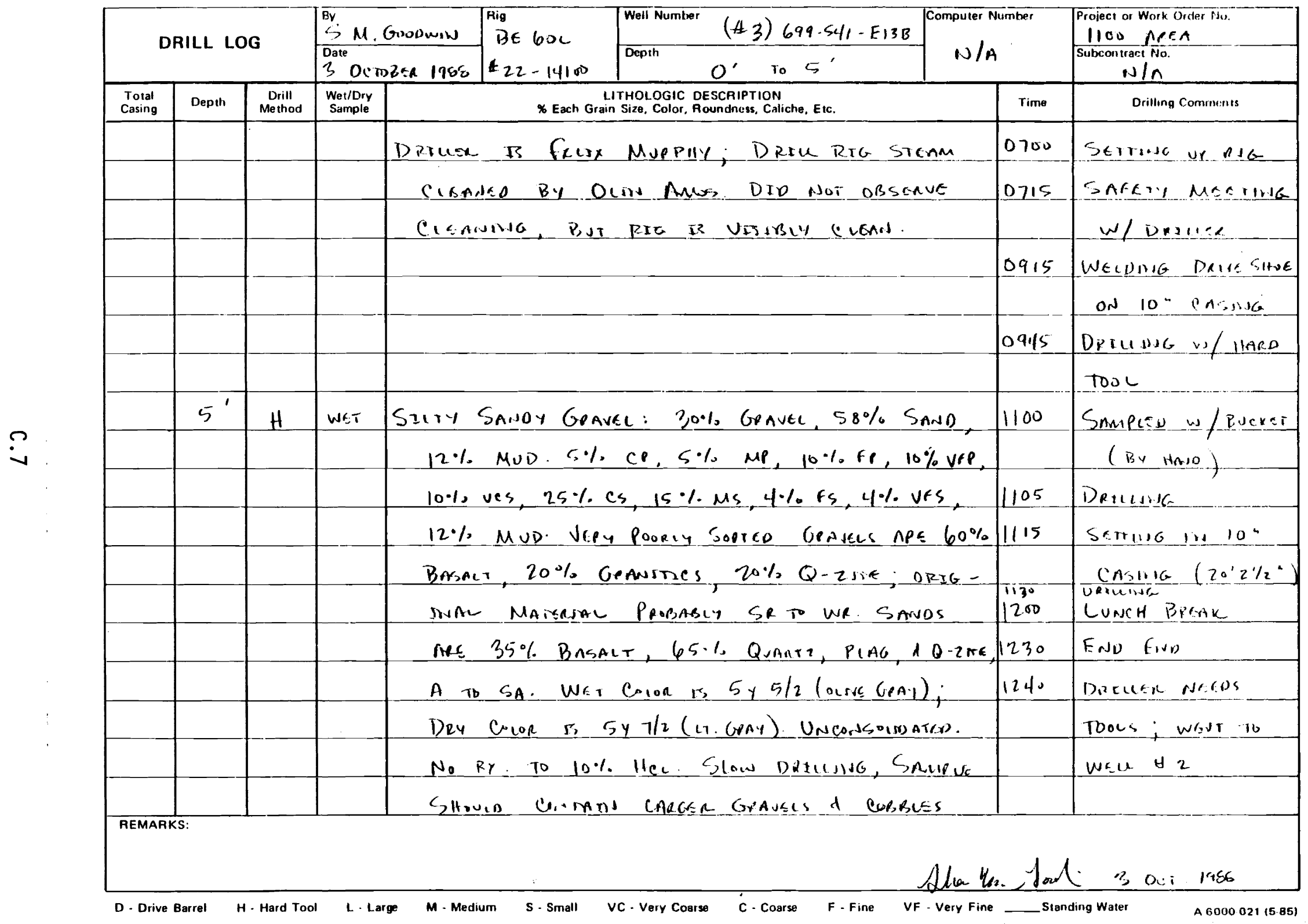


2 or 2

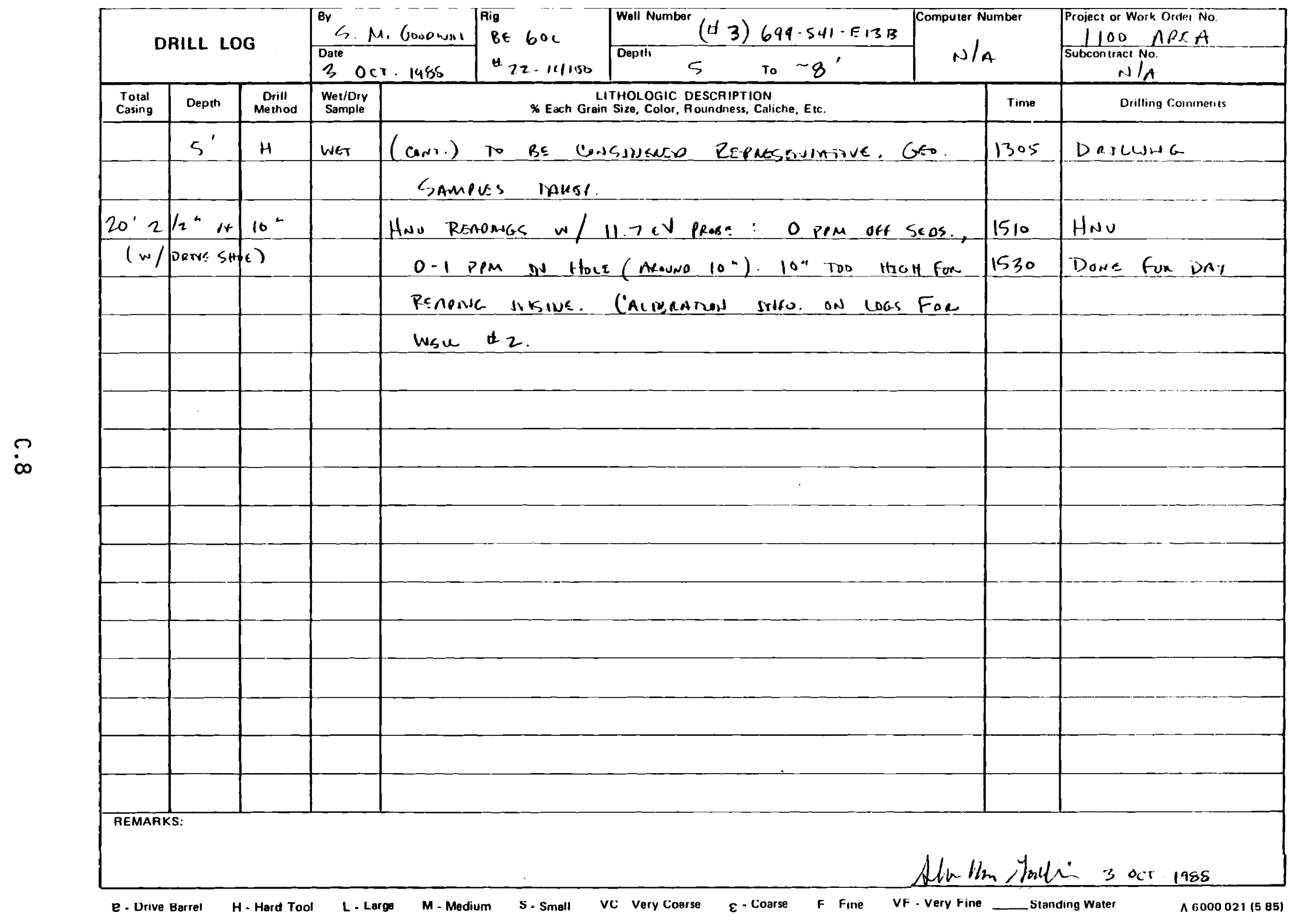


1 or 11

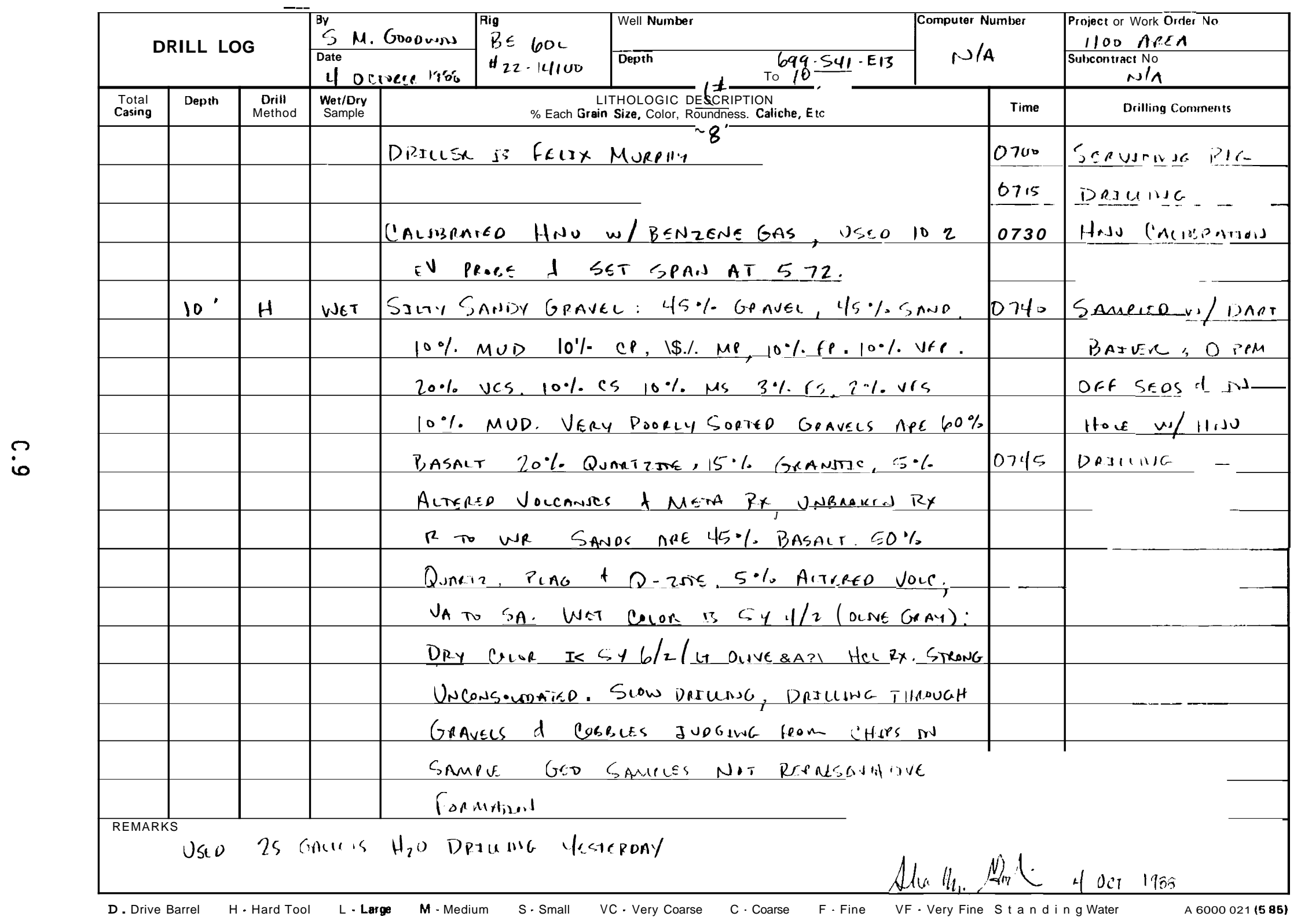


Cor 4

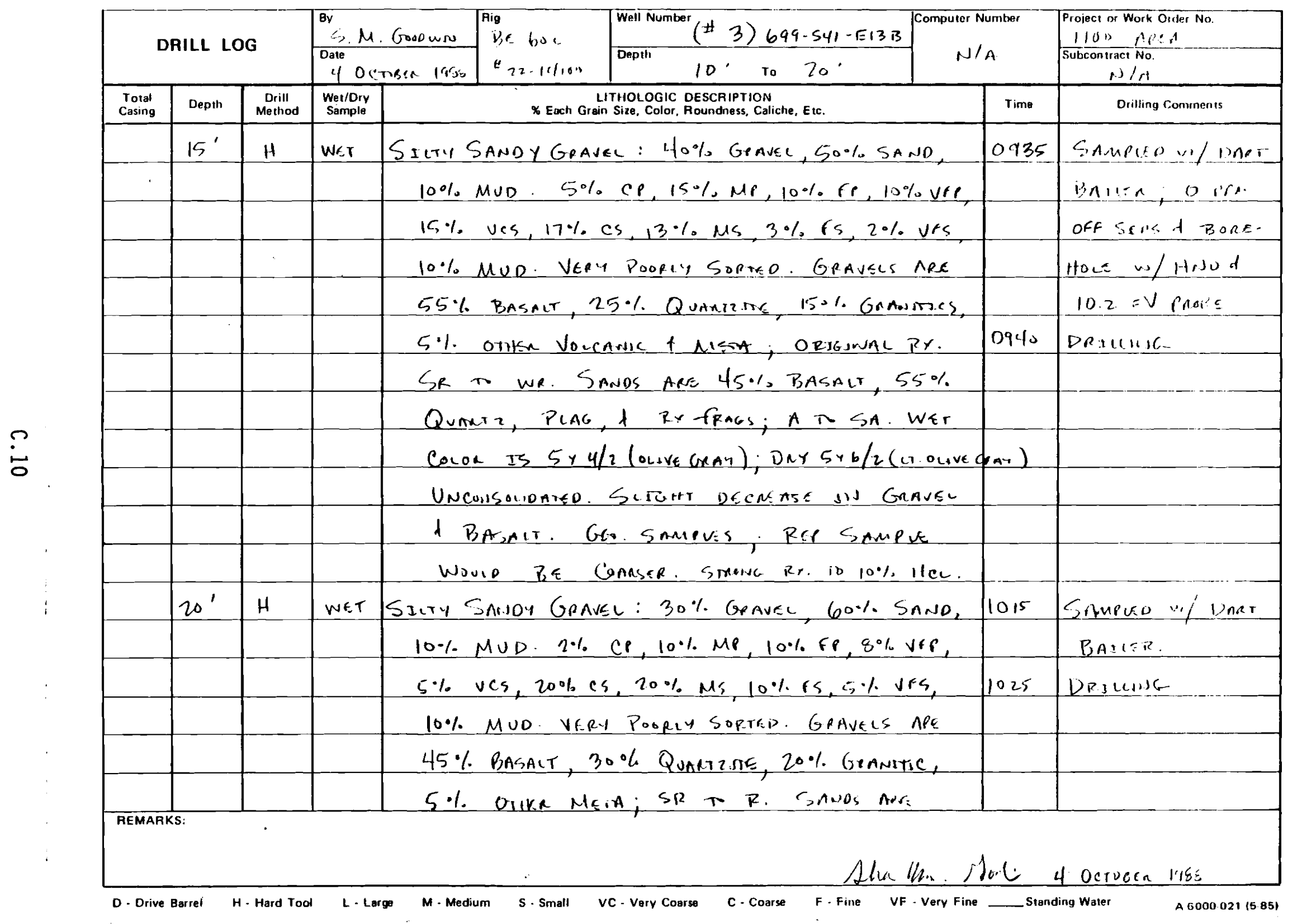




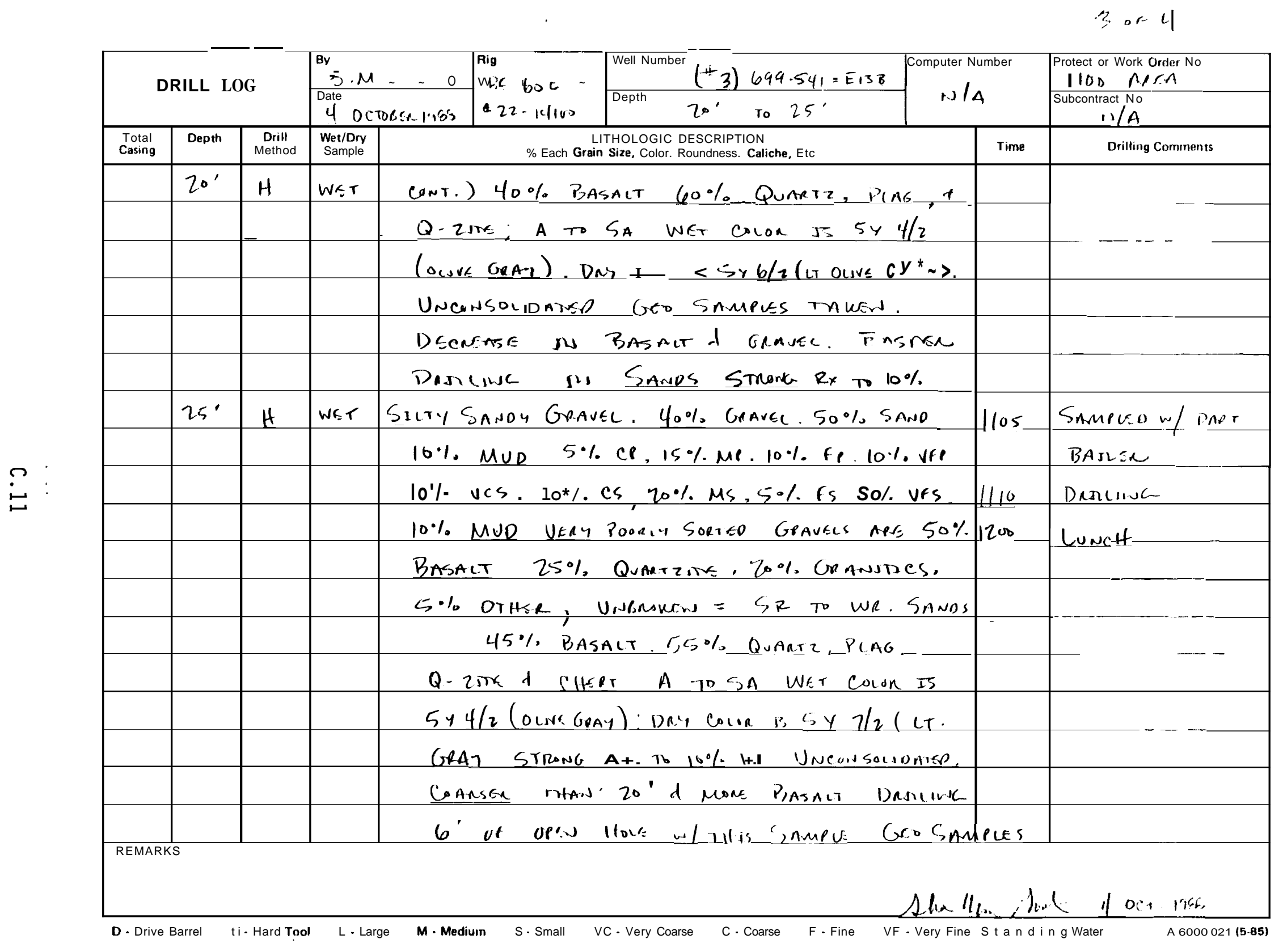


4 or 4

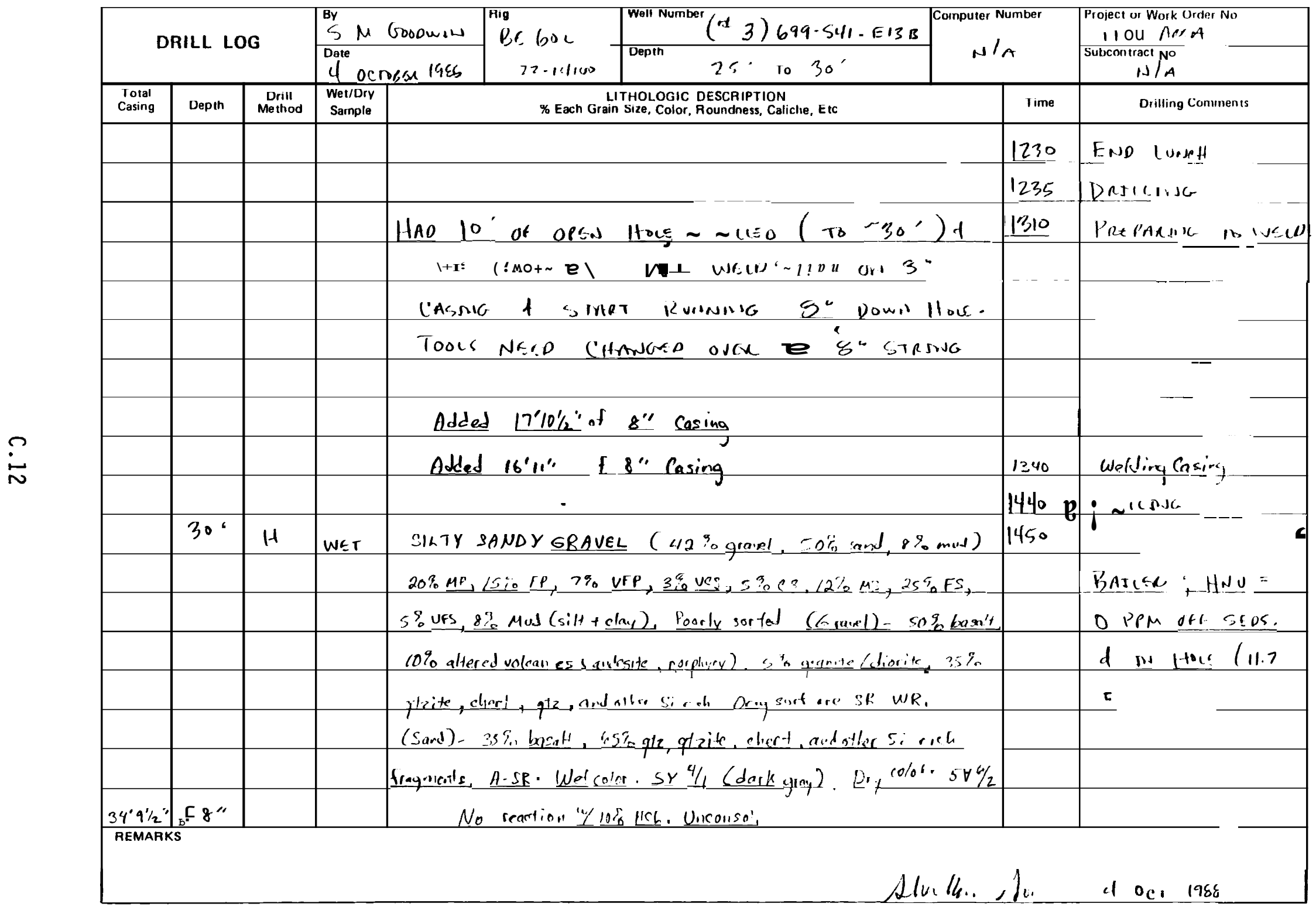

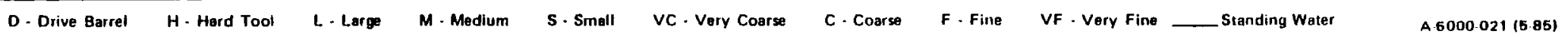


1 st 3

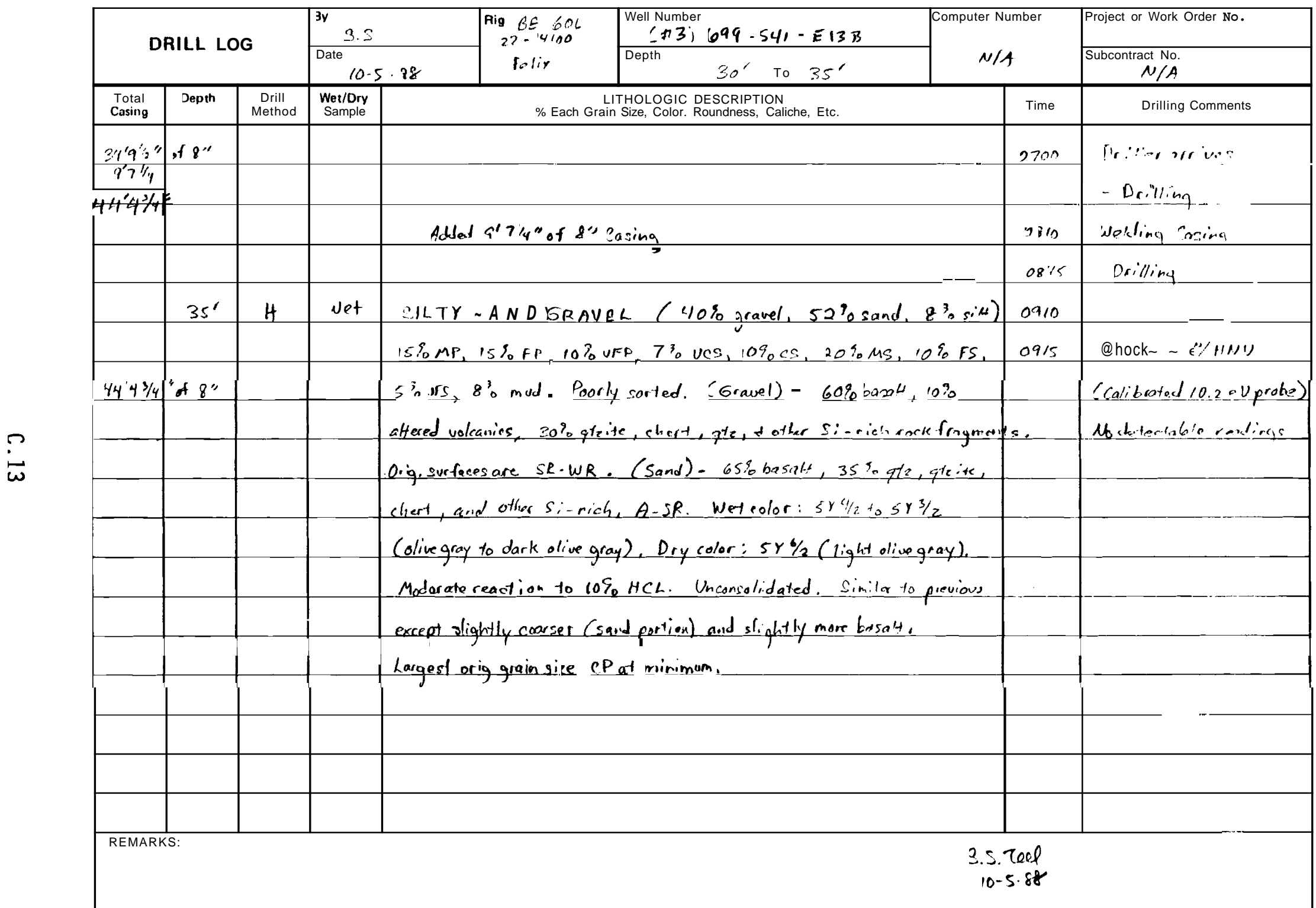


$\hat{n}, \hat{f} ?$

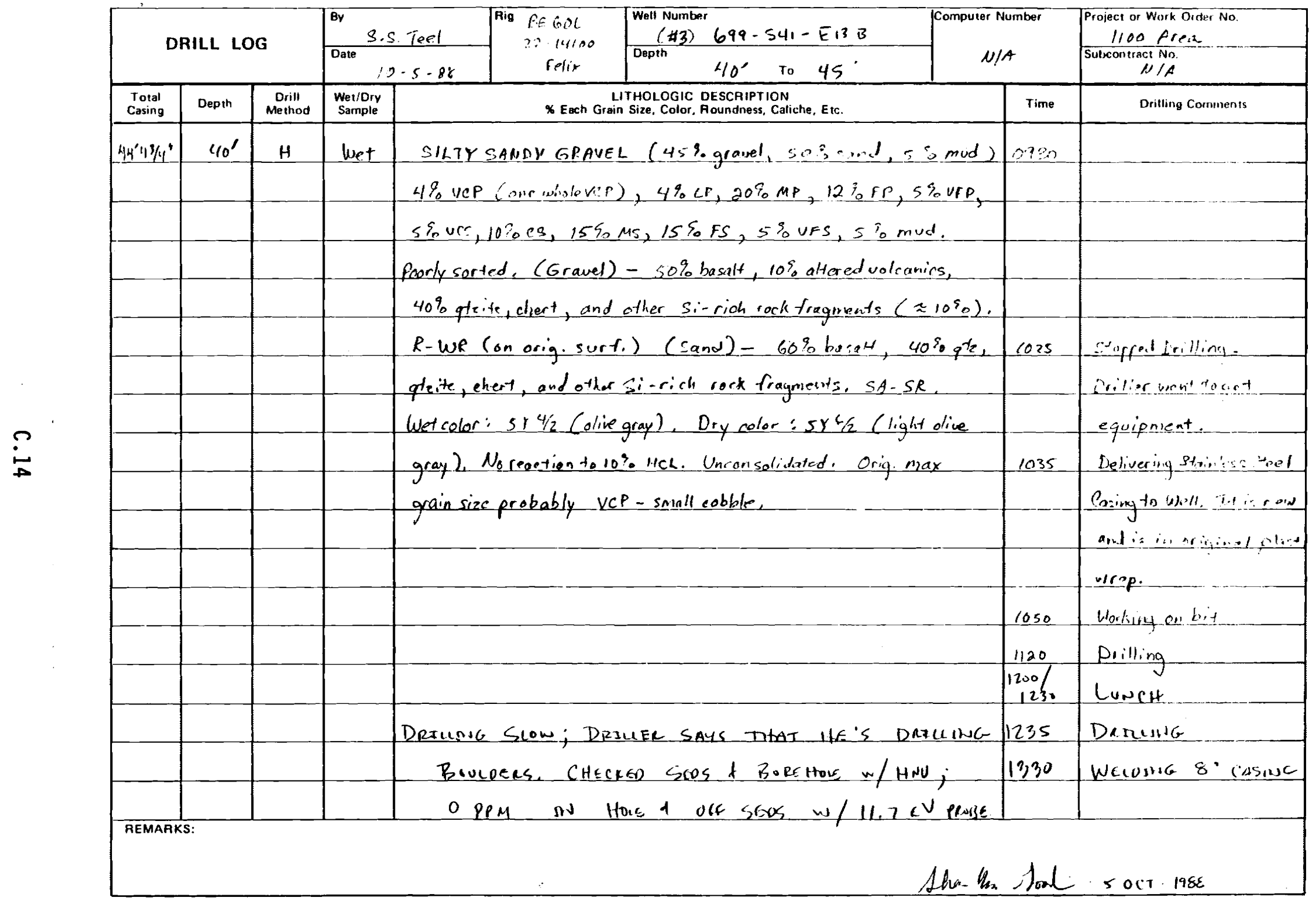

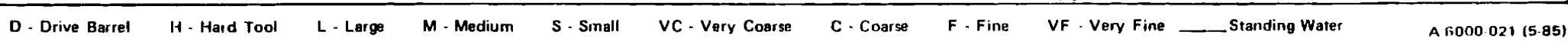


3 or?

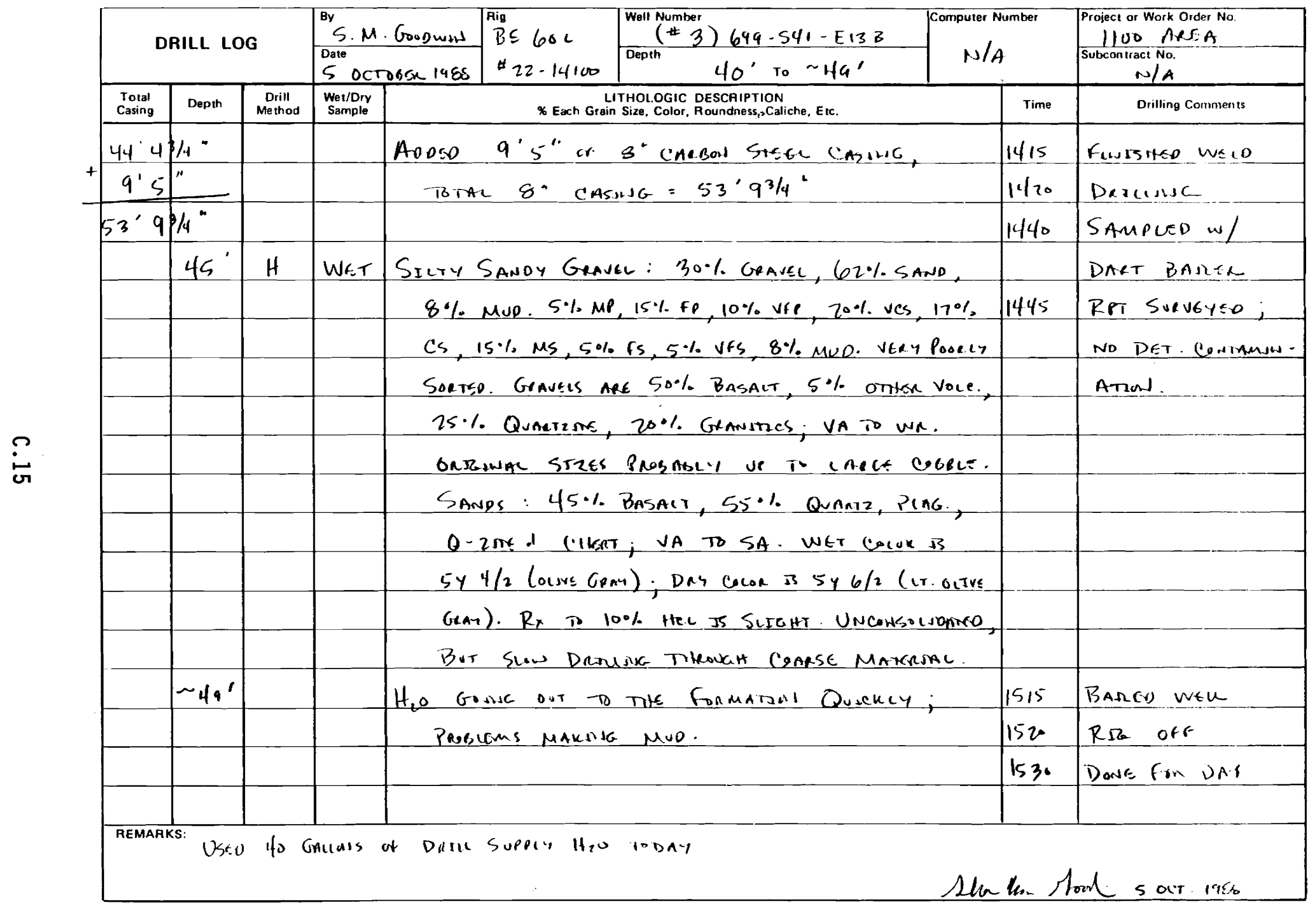

D:Drive Barrel H. Hard Toal L-Large M-Medium S-Small VC-Very Coarse C-Coarse F-Fine VF-Very Fine - Standing Water 


\begin{tabular}{|c|c|c|c|c|c|c|c|}
\hline & \multirow{2}{*}{\multicolumn{3}{|c|}{ DRILL LOG }} & \multirow{2}{*}{\begin{tabular}{|c|} 
S.M. Gooduns \\
Date \\
6 ocrobx 1988 \\
\end{tabular}} & \multirow{2}{*}{\multicolumn{2}{|c|}{\begin{tabular}{|c|c|c|}
$\begin{array}{c}\text { Well Number } \\
(33)\end{array} 699-541-513$ is \\
Depith & Computer Number \\
& $45^{\circ}$ To $50^{\prime}$ & \\
\end{tabular}}} & \multirow{3}{*}{$\begin{array}{l}\text { Project or Work Order No } \\
1100 \text { Mer } \\
\begin{array}{c}\text { Subcontraci Na. } \\
\text { N/A }\end{array} \\
\text { Drilling Comments }\end{array}$} \\
\hline & & & & & & & \\
\hline & $\begin{array}{l}\text { Total } \\
\text { Casing }\end{array}$ & Depth & $\begin{array}{c}\text { Drill } \\
\text { Method } \\
\end{array}$ & $\begin{array}{c}\text { Wet/Dry } \\
\text { Sample }\end{array}$ & $\begin{array}{l}\text { LITHOL OGIC DESCRIPTION } \\
\text { \% Each Grain Size. Color, Roundness, Caliche, Ex. }\end{array}$ & Time & \\
\hline & & & & & 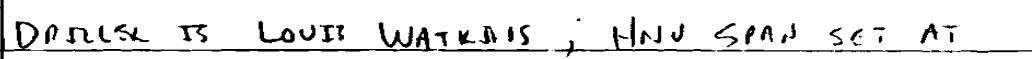 & 0700 & Seavienig in \\
\hline & & & & & 2.6 CaisRntso w/ P, ENzonse Cns. & 0710 & Dascesse \\
\hline & & $50^{\circ}$ & $H$ & WE.T & 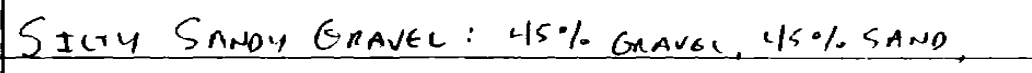 & 0740 & SAMPLCEO W/ DNT \\
\hline & & & & & $10 \%$ Mup $5 \% C^{P}, 10 \% \mathrm{MP}, 15 \%$ Fe, $15 \%$ v $=0$, & & Bajer \\
\hline & & & & & $15 \%$ ves, $10 \%$ cs, $10 \%$ Ms, $5 \%$ Fs, $5 \%$ vas, & $07 \cdot 15$ & Das..., \\
\hline & & & & & $10 \%$ MUD. VEAY PoORL SORKOD. GRAVELS & o8as & 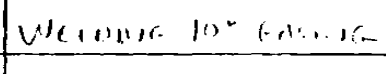 \\
\hline & & & & & $40 \%$ BDASACT, $5 \%$ ACT VOCCMNICS, $30 \%$ & & Driller say = Rimpoll e \\
\hline & & & & & Qunarzms, $25 \%$ GPANSTCS; A A WR. & & $\leqslant n^{\prime}$ \\
\hline & & & & & SA.19S: $35 \%$ BASALT, $65 \%$ QUARTZNE, QUMCTZ & & \\
\hline & & & & & CIler, PIAG; VA TD SA WET CheOn IS & & \\
\hline & & & & & 5y 3/2 (Darn olve Gray); Day colok is $5 y$ & & \\
\hline & & & & & 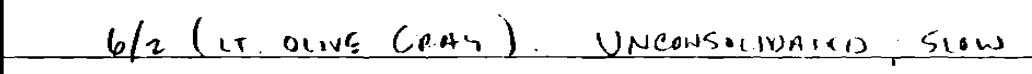 & & \\
\hline & & & & & 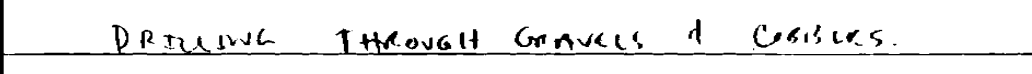 & & \\
\hline & & & & & 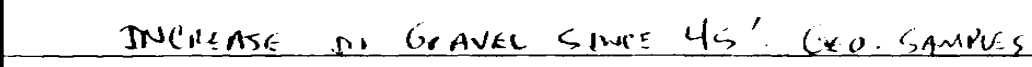 & & \\
\hline & $533^{\prime} 9$ & $3 / 4^{n}$ & & & TAver & & \\
\hline+ & $8^{\prime} 4$ & 4 & & & 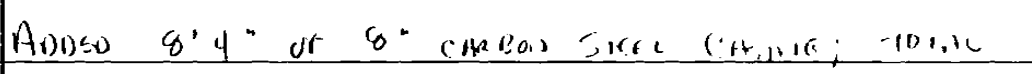 & 0920 & finsalles vieno \\
\hline & 62 & $3 / 4^{\prime \prime}$ & & & $8^{\circ} \operatorname{cone}=62^{\prime} 1^{3 / 4}$ & 0425 & DRSMUG \\
\hline & & & & & Lo Drm or SEDS. A IN AnNueus; 11,2 EV procse & 10955 & HNU RSnOHG: \\
\hline & FEMARK: & & & & 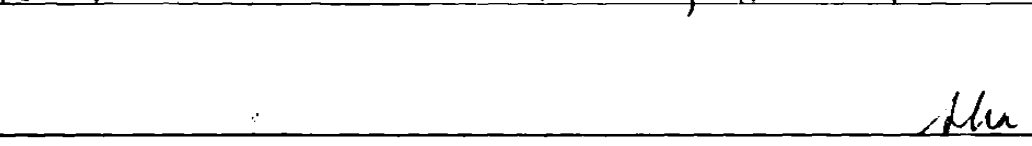 & 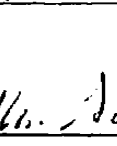 & (. 6 oc. res \\
\hline
\end{tabular}


2 of 4

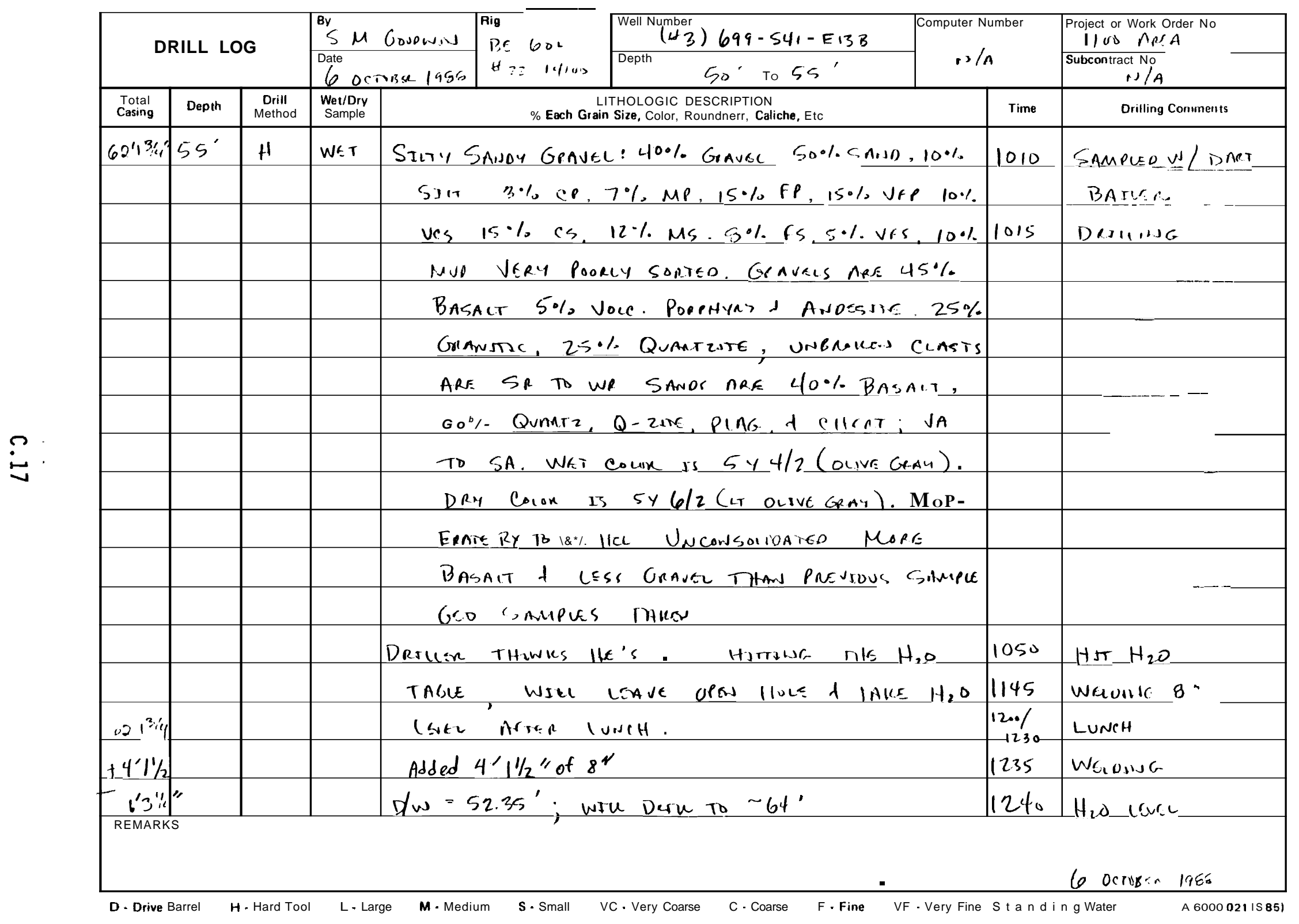


2. if

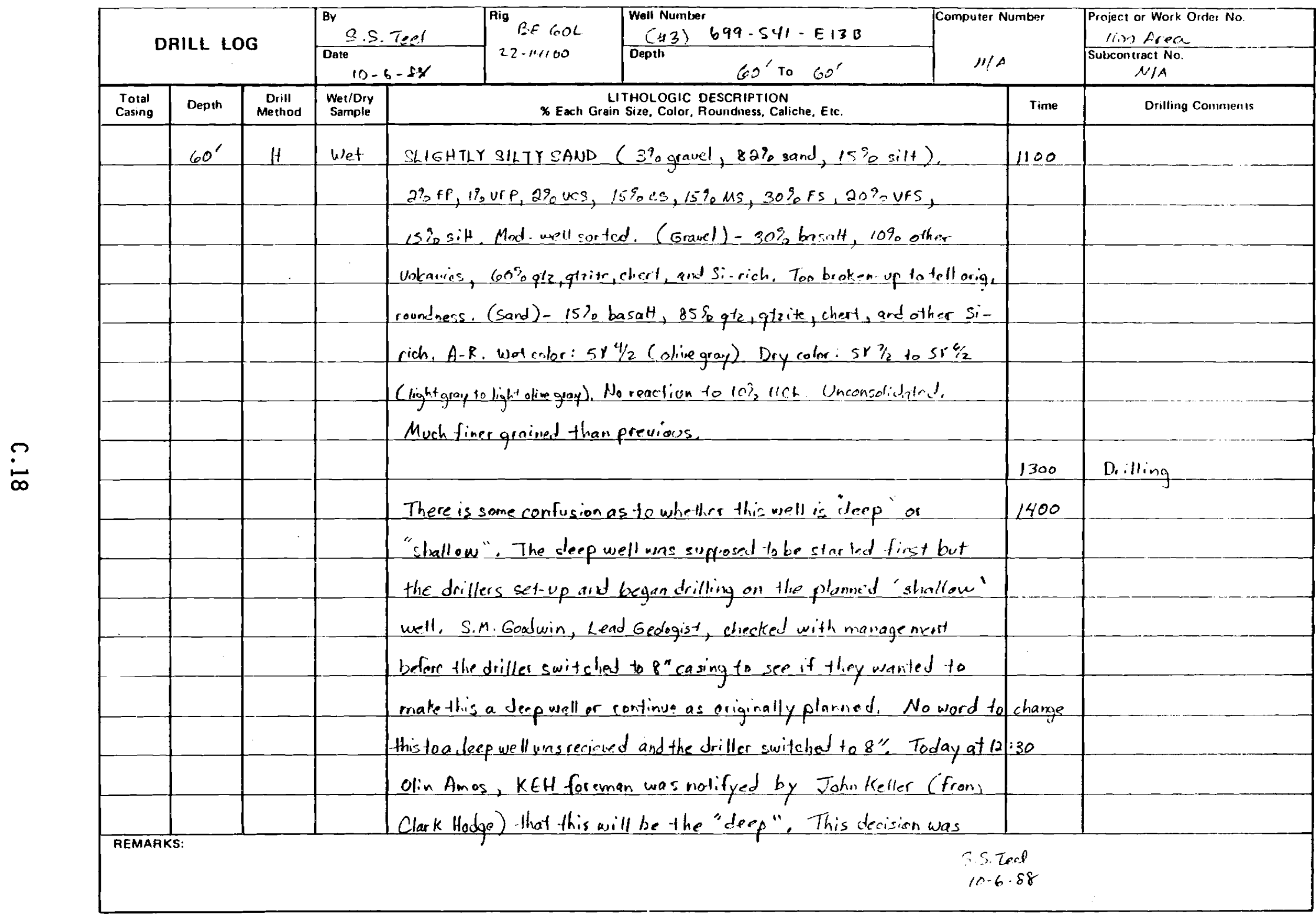

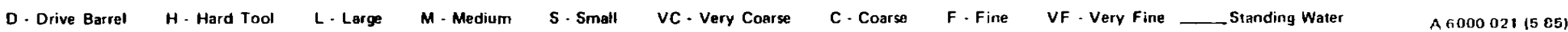


Lif of 4

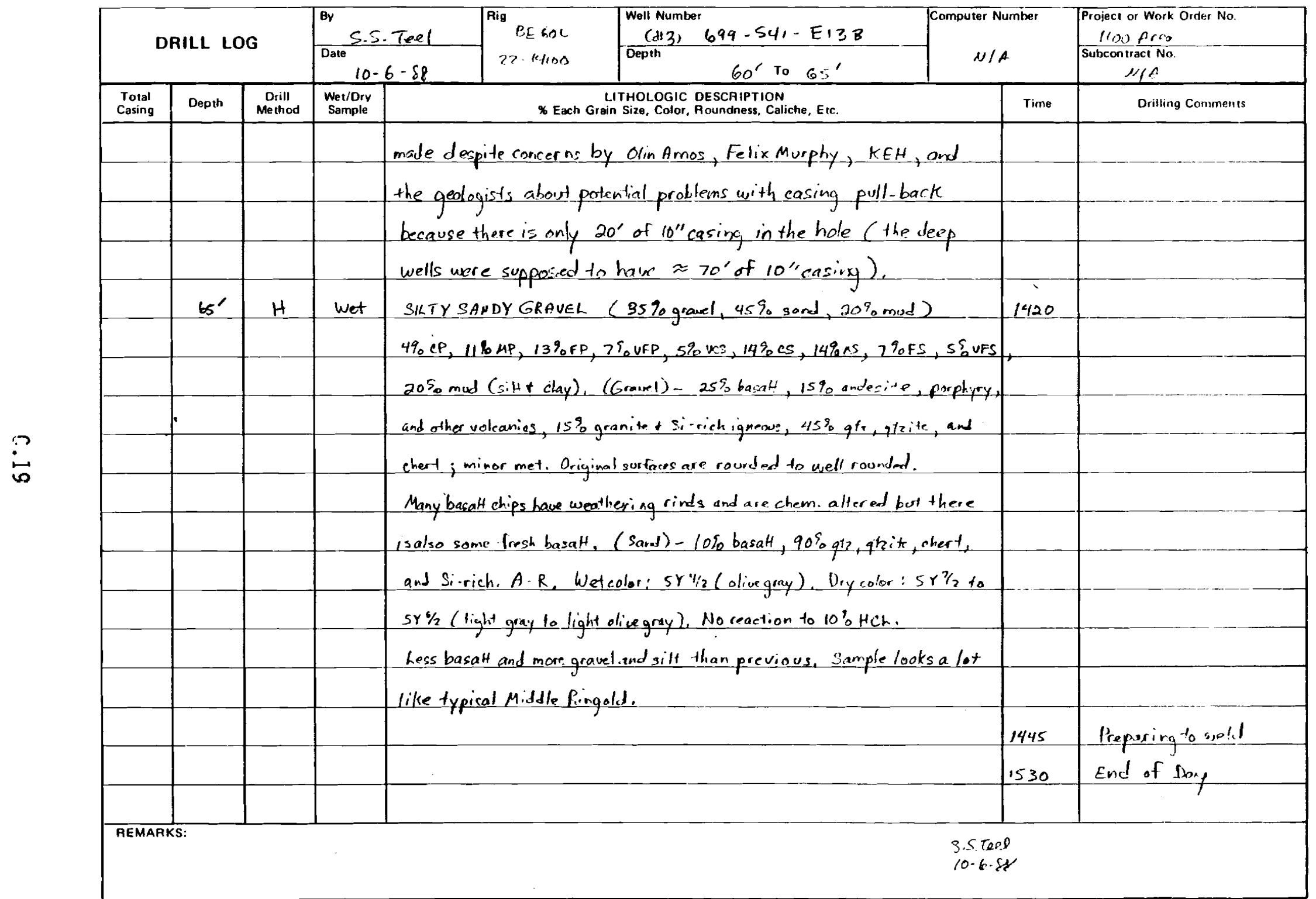

D. Drive Barrel H. Hard Tool L. Large M. Medium S. Small VC - Very Coarse C. Coarse F. Fine VF - Very Fine _ Standing Water 


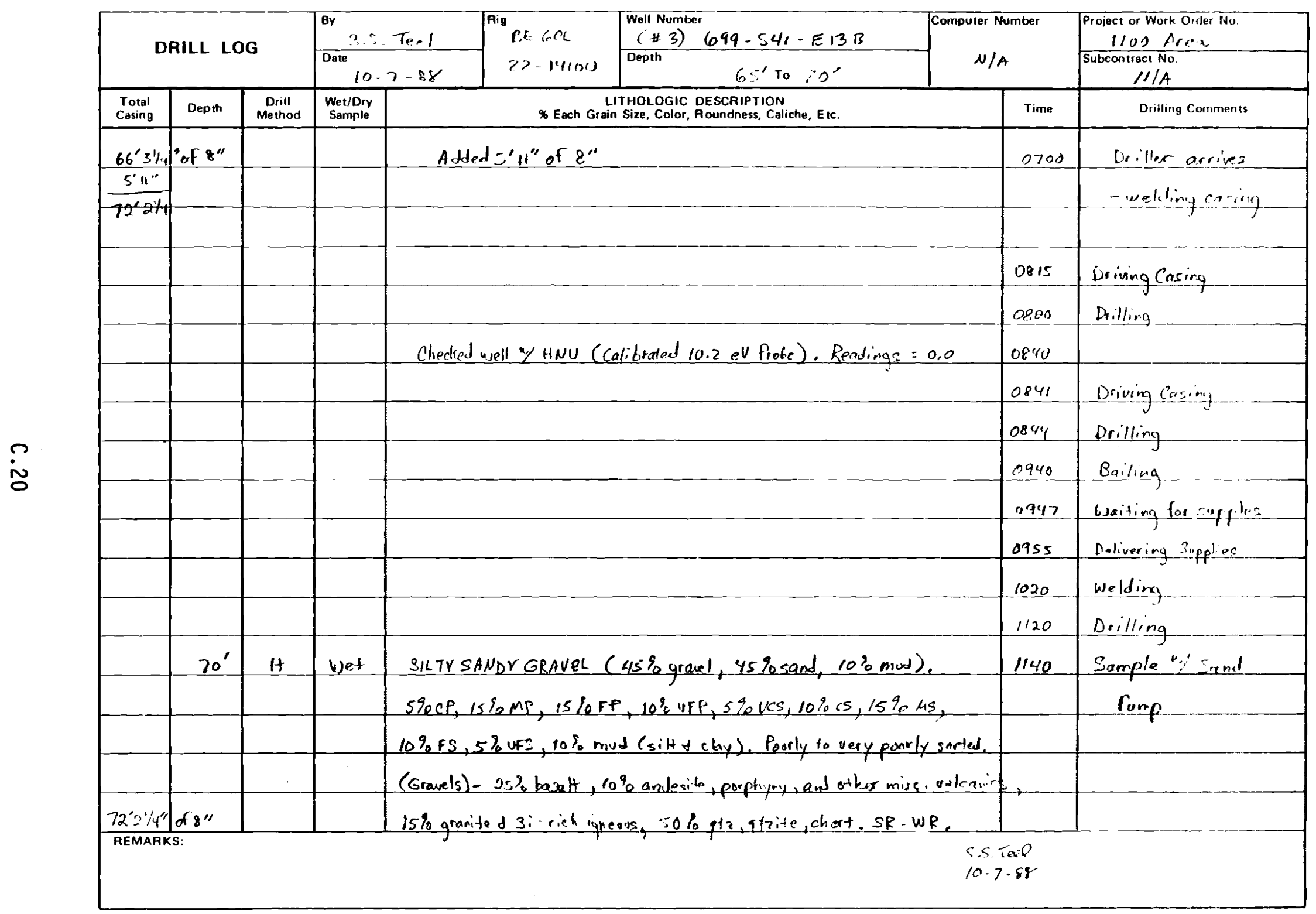

D. Drive Barrel H- Hard Tool L. Large M-Medium S-Smell VC-Very Coarse C. Coarse F. Fine VF-Very Fine Standing Water A tiouo 021 (5.85) 
2 of 2

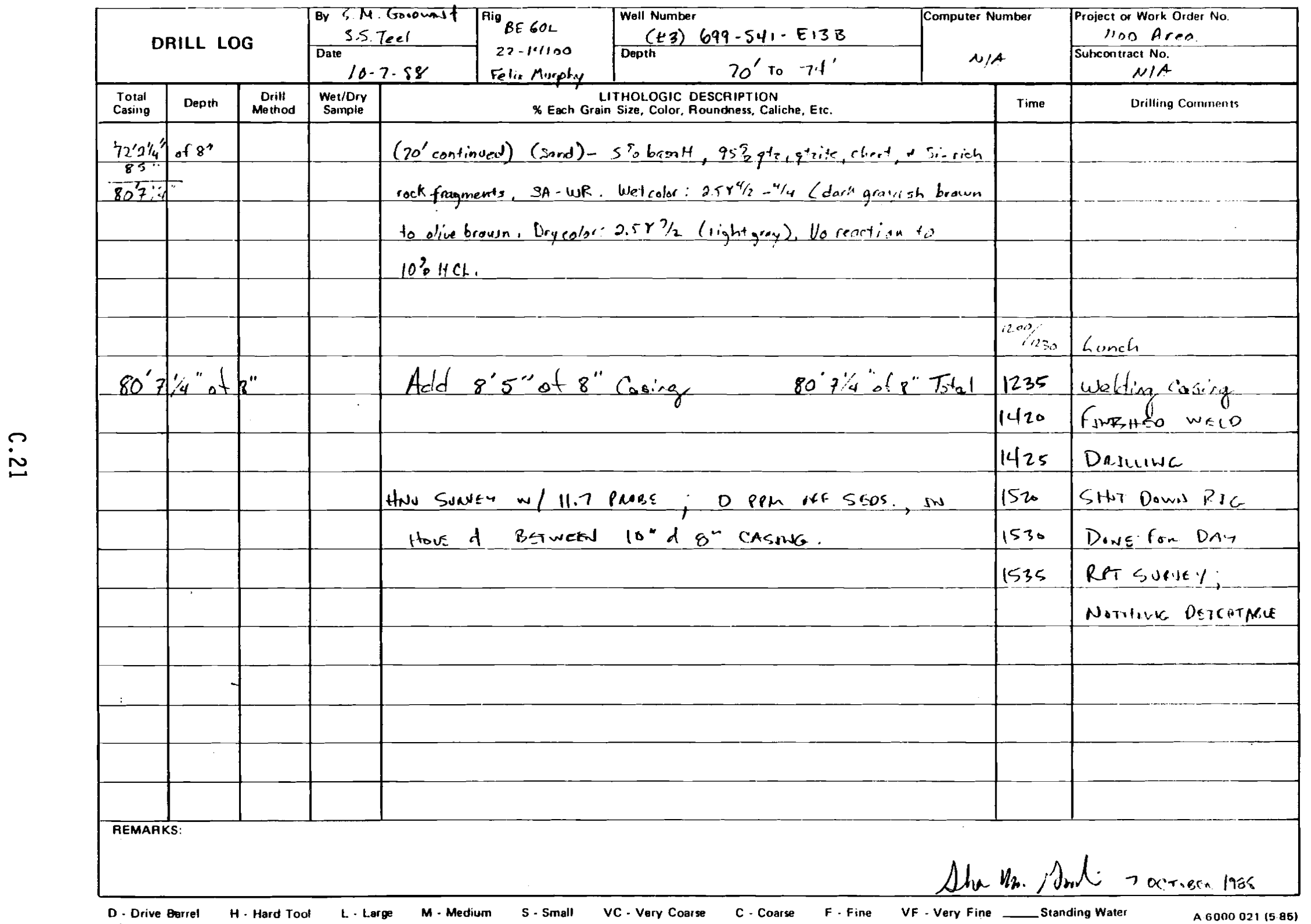


1 of 4

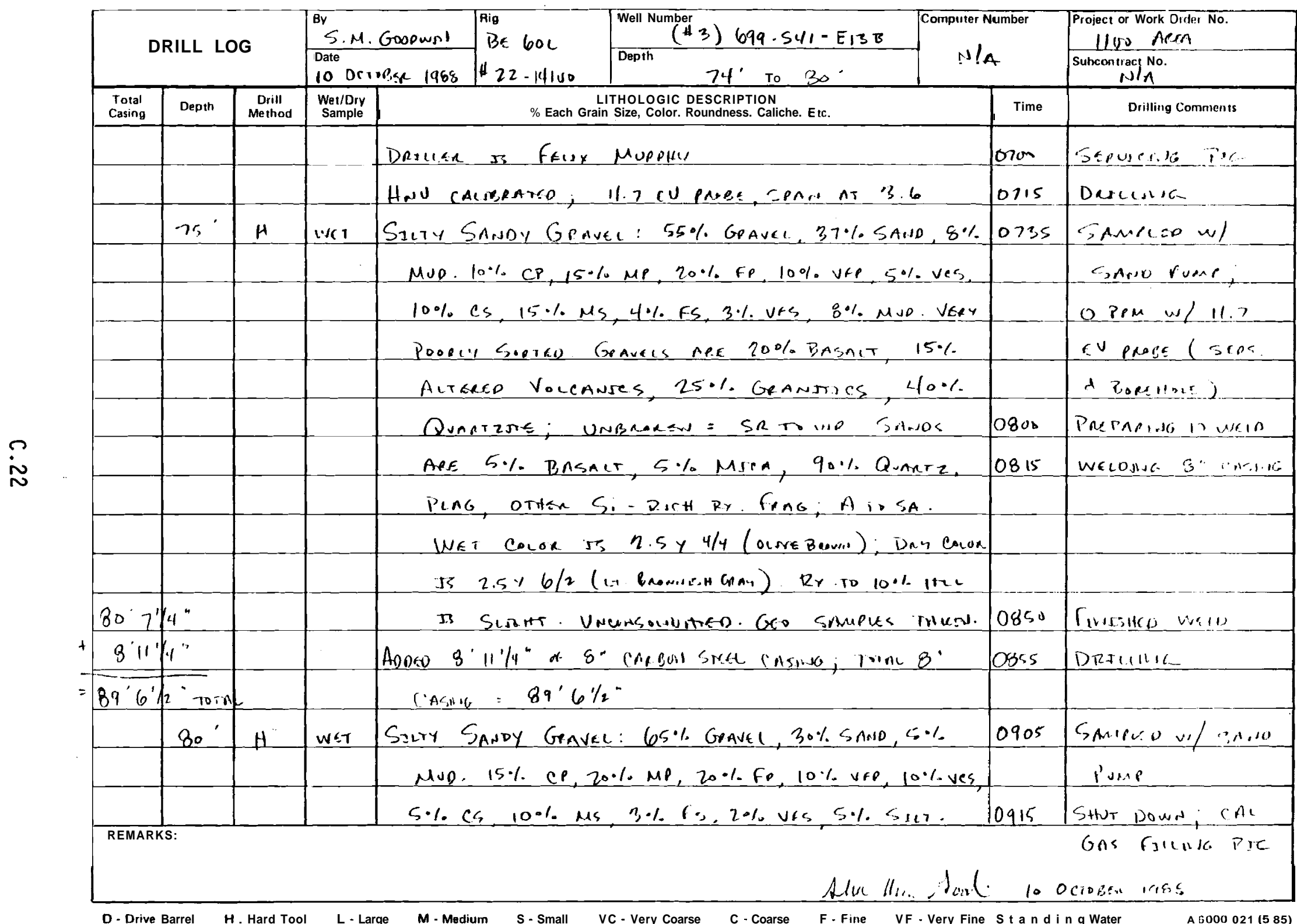





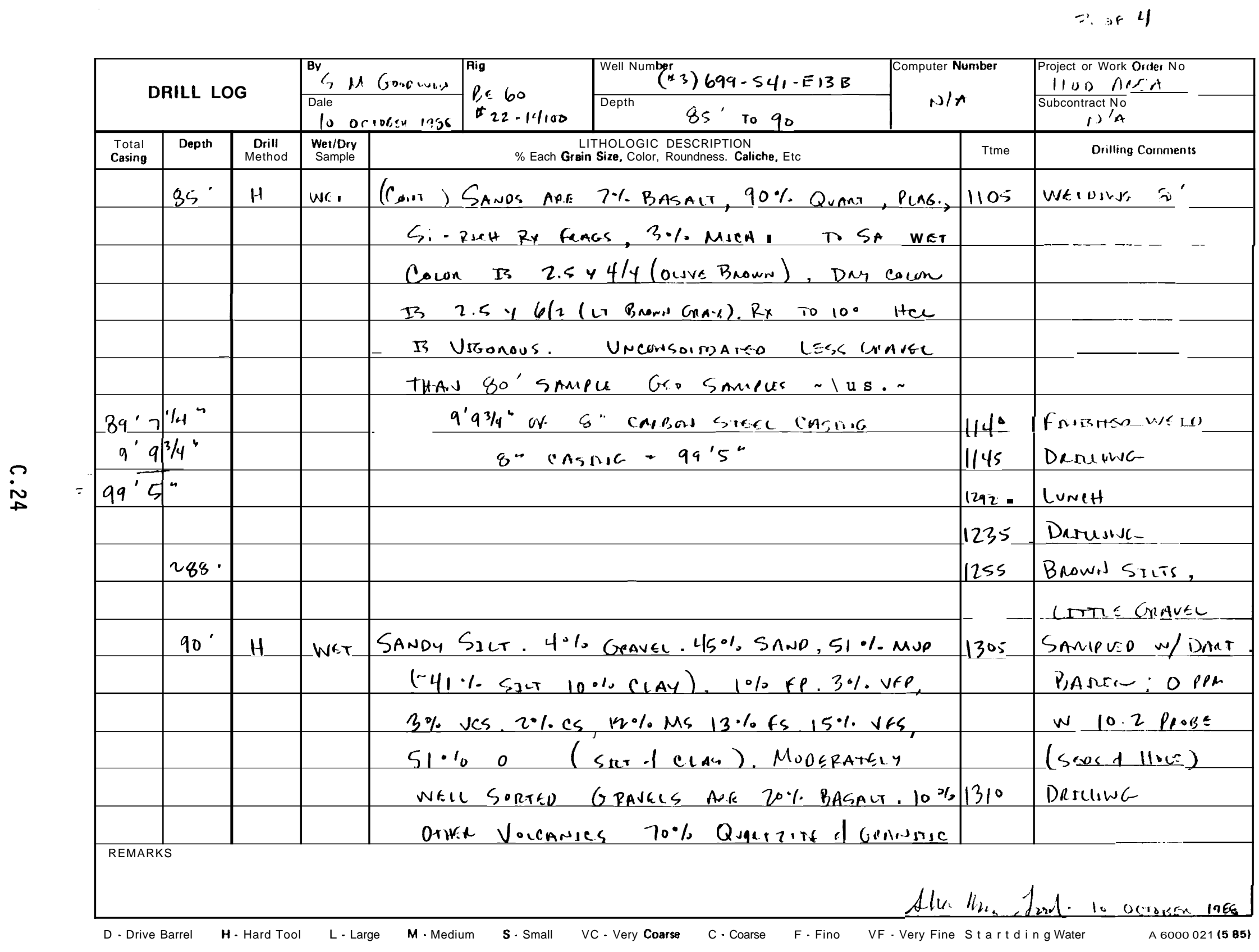


4 of 4

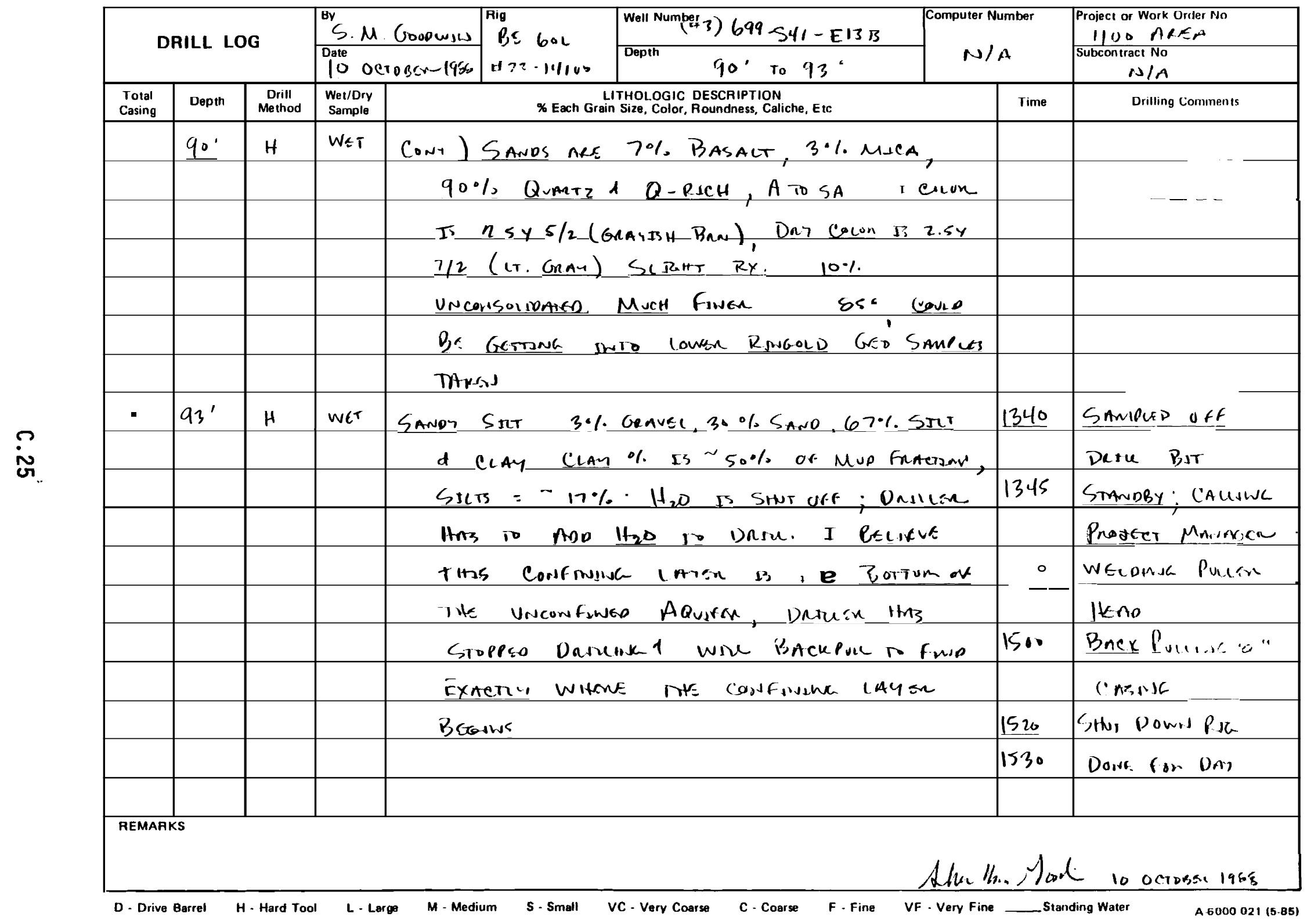


1 or 2

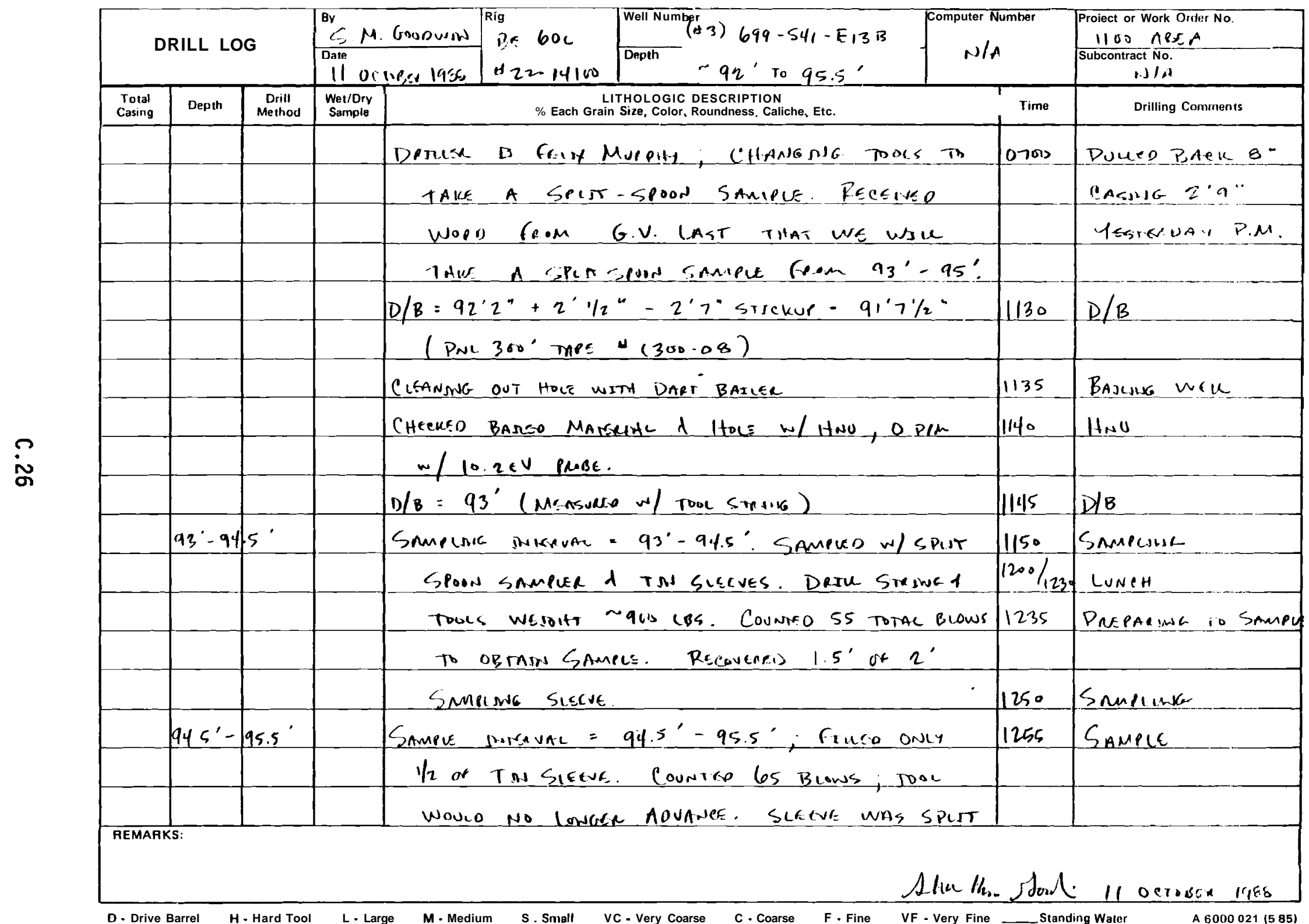


2 or 2

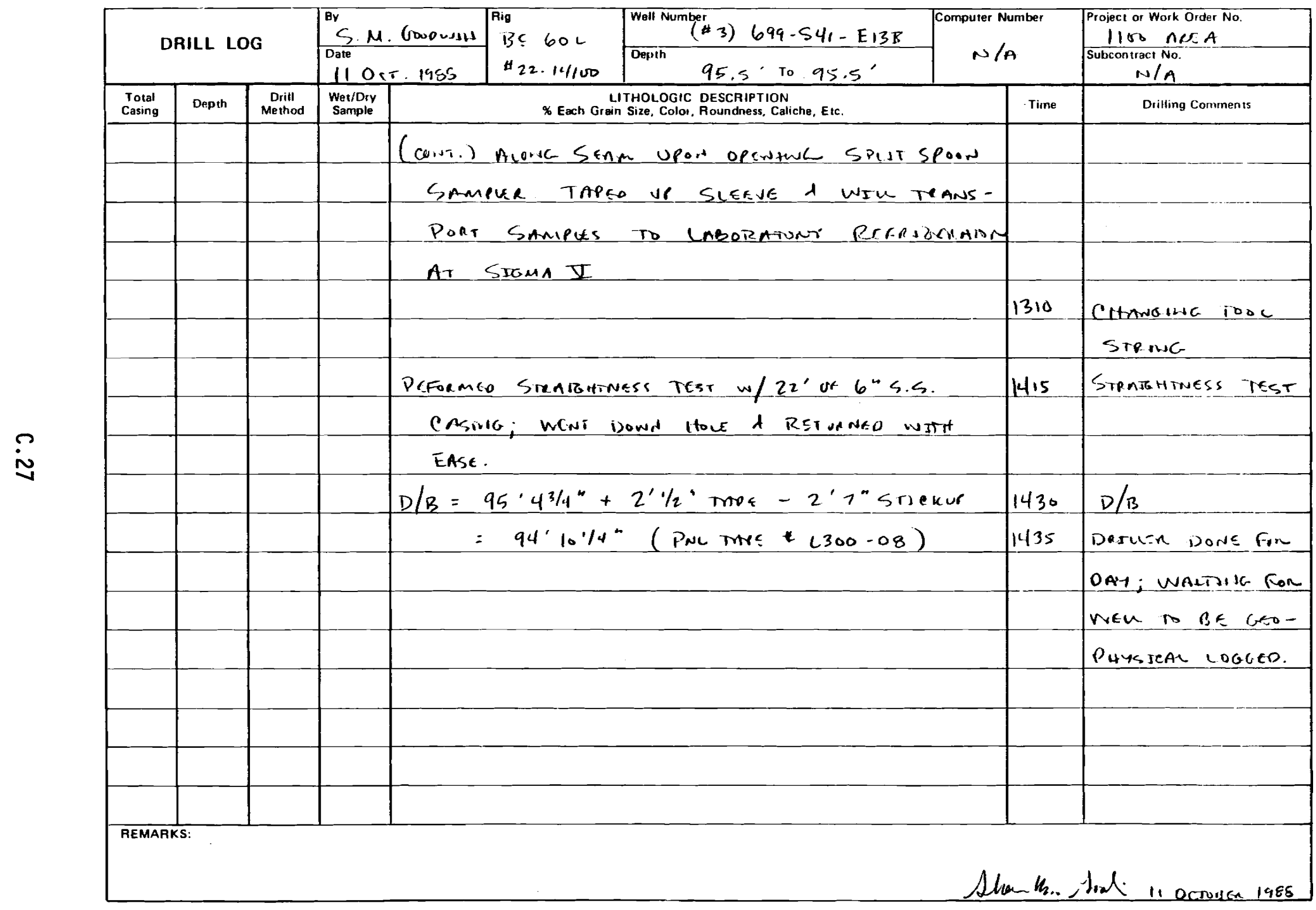

D. Drive Barrel H- Hard Tool L-Lerge M-Medium S-Small VC - Very Coorse C-Coarse F-Fine VF - Very Fine — Standing Water A 
1 ot 2

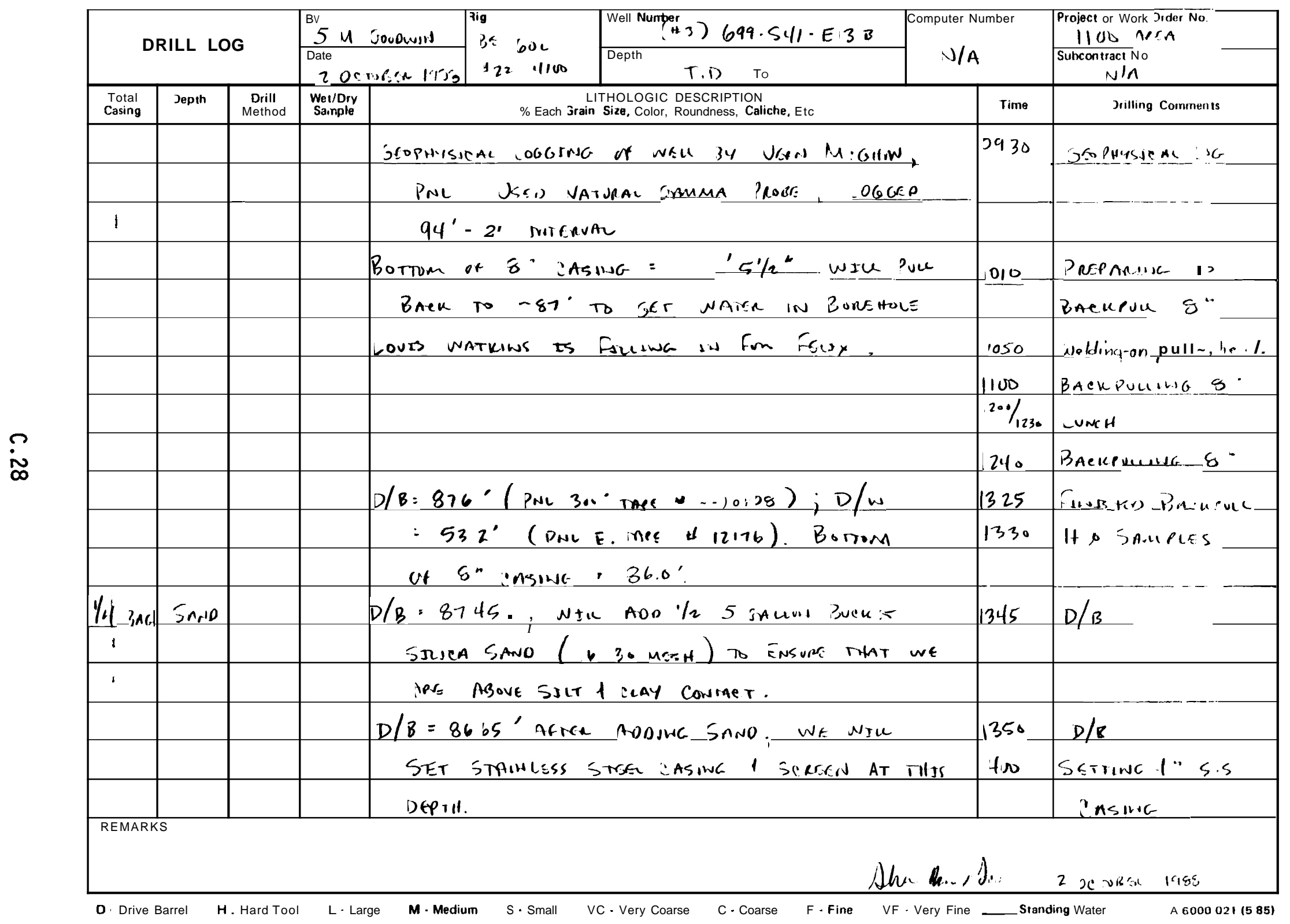


2 . 2

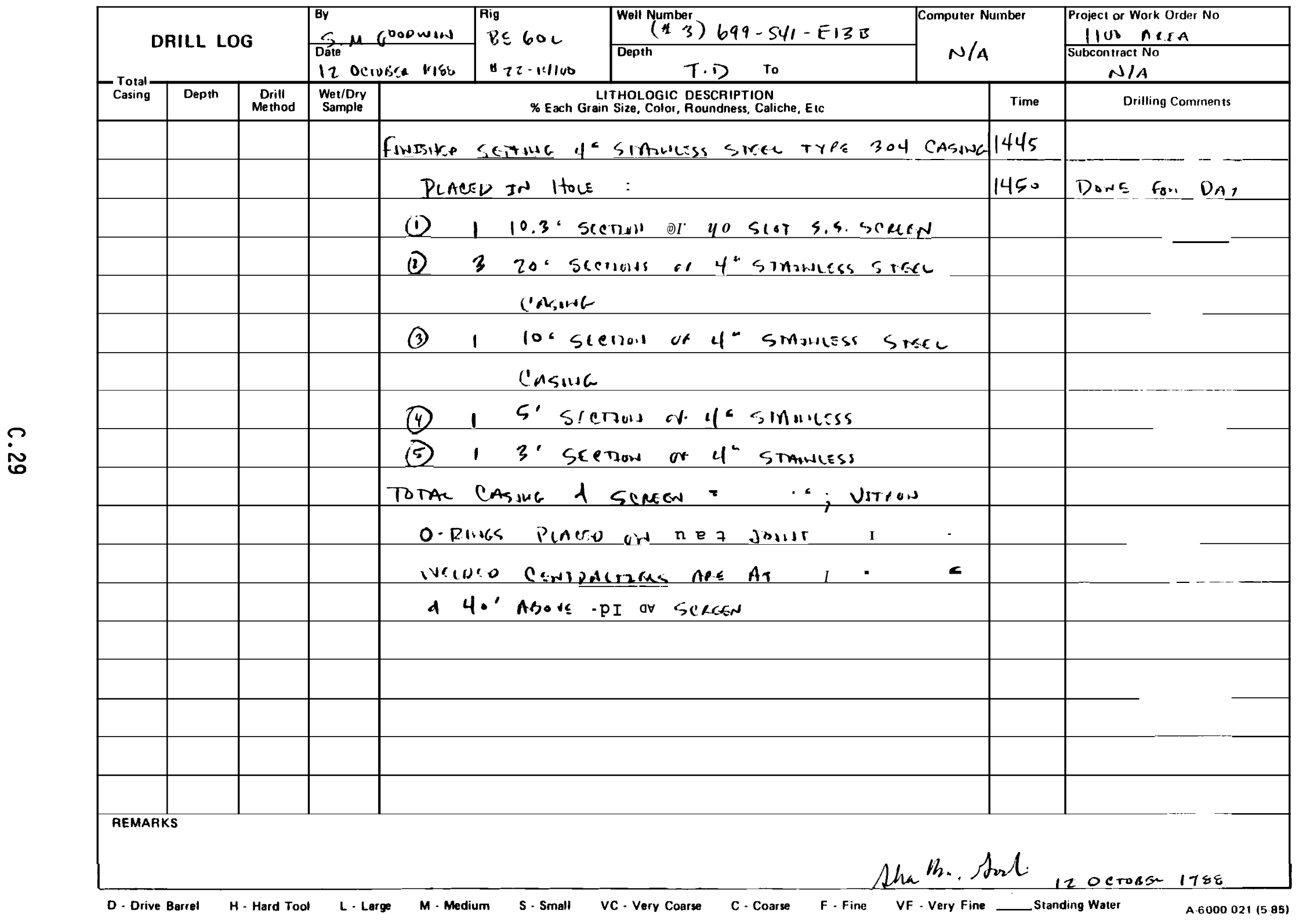




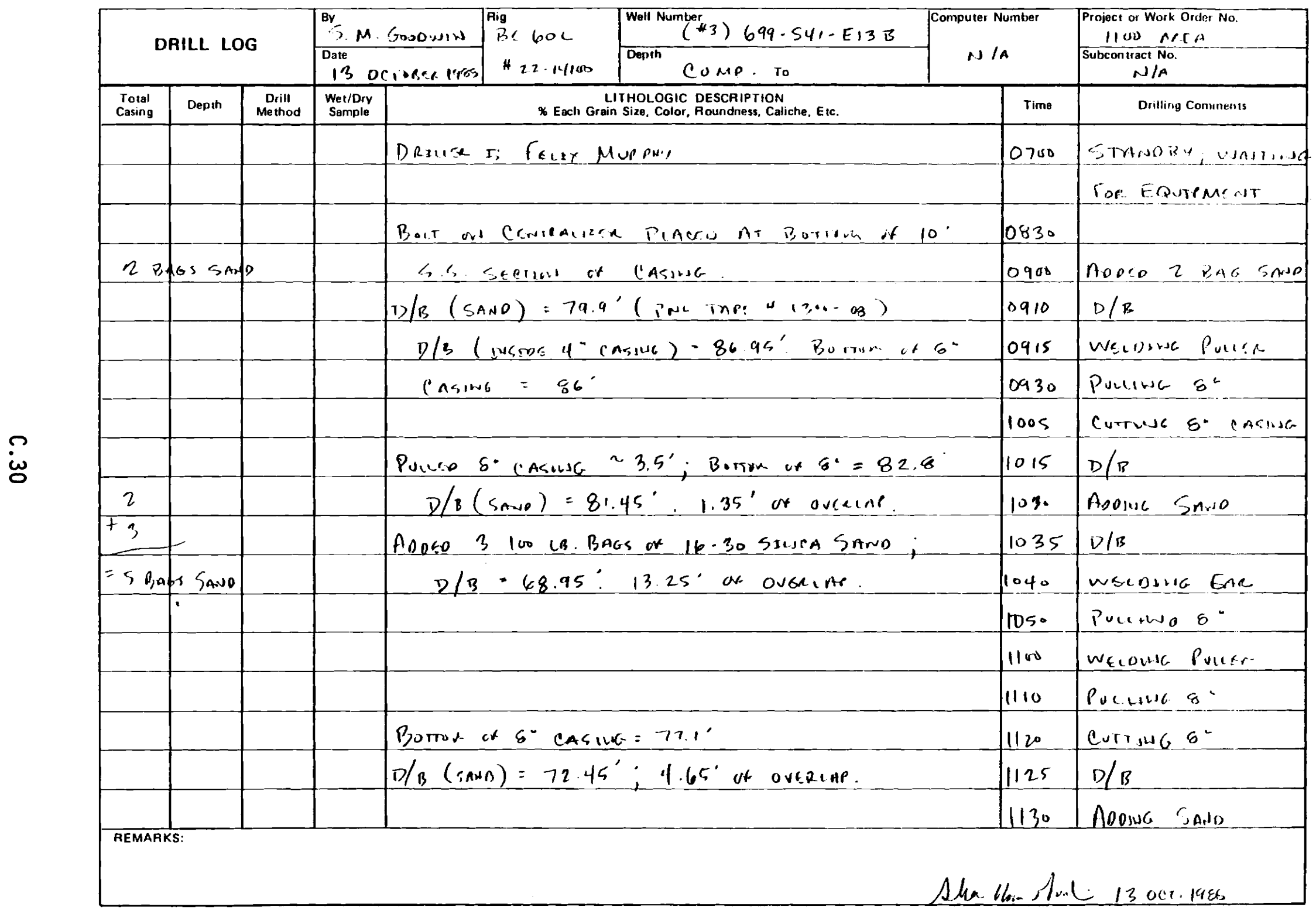

D. Drive Barral H-Hard Tool L-Large M-Medium S-Small VC-Very Coarse C-Coarse F-Fine VF-Very Fine _-Standing Water A 
$2 \cdot 43$

\begin{tabular}{|c|c|c|c|c|c|c|c|}
\hline \multirow[b]{4}{*}{+} & \multicolumn{3}{|c|}{ DRILL LOG } & $\begin{array}{l}\text { By M. Gooowis } \\
13 \text { ocronse 1956 }\end{array}$ & \multicolumn{2}{|l|}{\begin{tabular}{|c} 
Well Num ber' \\
Depth \\
Conif $699-541-138$ \\
\end{tabular}} & \multirow{2}{*}{$\begin{array}{c}\text { Proiect or Work Order No. } \\
1 / \text { in A A A A } \\
\begin{array}{c}\text { Subcontract No } \\
\text { N/A }\end{array} \\
\text { Diilling Comenents }\end{array}$} \\
\hline & $\begin{array}{c}\text { Total } \\
\text { Casing } \\
\end{array}$ & Depth & $\begin{array}{c}\text { Drill } \\
\text { Method }\end{array}$ & $\begin{array}{l}\text { Wet/Dry } \\
\text { Sample } \\
\end{array}$ & $\begin{array}{r}\text { LITHOLOGIC DESCRIPTION } \\
\text { \% Each Grain Size, Color, Roundness. Caliche, Etc }\end{array}$ & Time & \\
\hline & 5 BAs: & & & & $D / P=70.05^{\prime} ; 7.05^{\prime}$ at osectine Anosed $1 / 2100$ & 1135 & $D / B$ \\
\hline & $1 / 2$ & & & & (1). P:AG oK 16.30 MESII SAND. & 1140 & WEDDWE PULCR \\
\hline$=$ & $51 / 2$ & & & & & 1150 & Pounde 8 - \\
\hline+1 & $1 / 4(1$ & $10 / 12)$ & & & & $1200 / 1230$ & LUNCH \\
\hline$=$ & $53 / 4$ B 34 & (6) Tor & ter sined & & & 1235 & Whions End \\
\hline & & & & & & 1245 & Poumenc 8 . \\
\hline & & & & & Puurs 8" CAsing $4^{\prime}$; Borrum of $8^{\circ}=73.1^{\prime}$ & 1255 & Currunc s. \\
\hline & & & & & $D / B($ Sawo $)=72.0^{\prime} ; 3.1^{\prime}$ os overins. Tor & 1305 & $D / B$ \\
\hline & & & & & of Smud PACK ; $14.95^{\circ}$ at SANO PaCK. SAND & 1315 & Runasug TERouse \\
\hline & & & & & 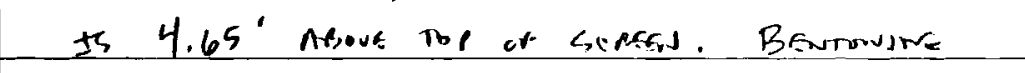 & & DIPS DOW'S HOLE. \\
\hline & & & & & Geost well BE TRrmaks Down Hove to & & \\
\hline & & & & & '2'ABOUE THE HZO TABLE. & & \\
\hline & & & & & 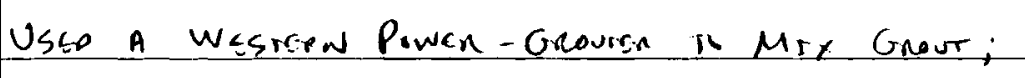 & 1330 & 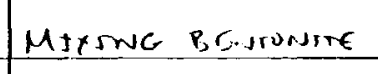 \\
\hline & & & & & USED I UD UB. BAG of POWDSaED BR HORS & & Geout \\
\hline & & & & & 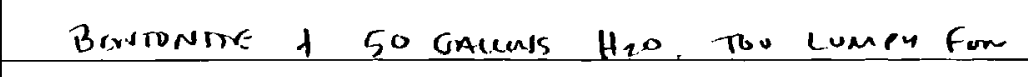 & 1345 & Poverne Grout \\
\hline & & & & & Malsh furres Viscorim. & & \\
\hline & & & & & $D / B($ GPOVT $)=49.65^{\circ} ; 204^{\prime}$ of OVERIAP. & 1350 & $D / B$ \\
\hline & & & & & & 1355 & Puence Tormse \\
\hline & & & & & $\Delta h a$ & Mos & $=13 \mathrm{ocr} \cdot 195 \mathrm{~S}$ \\
\hline
\end{tabular}

D. Drive Barrel H. Hard Tool L. Large M-Medium S.Small VC. Very Coarse C.Coarse F. Fine VF. Very Fine Stand ing Water A6000 021 (5.95) 


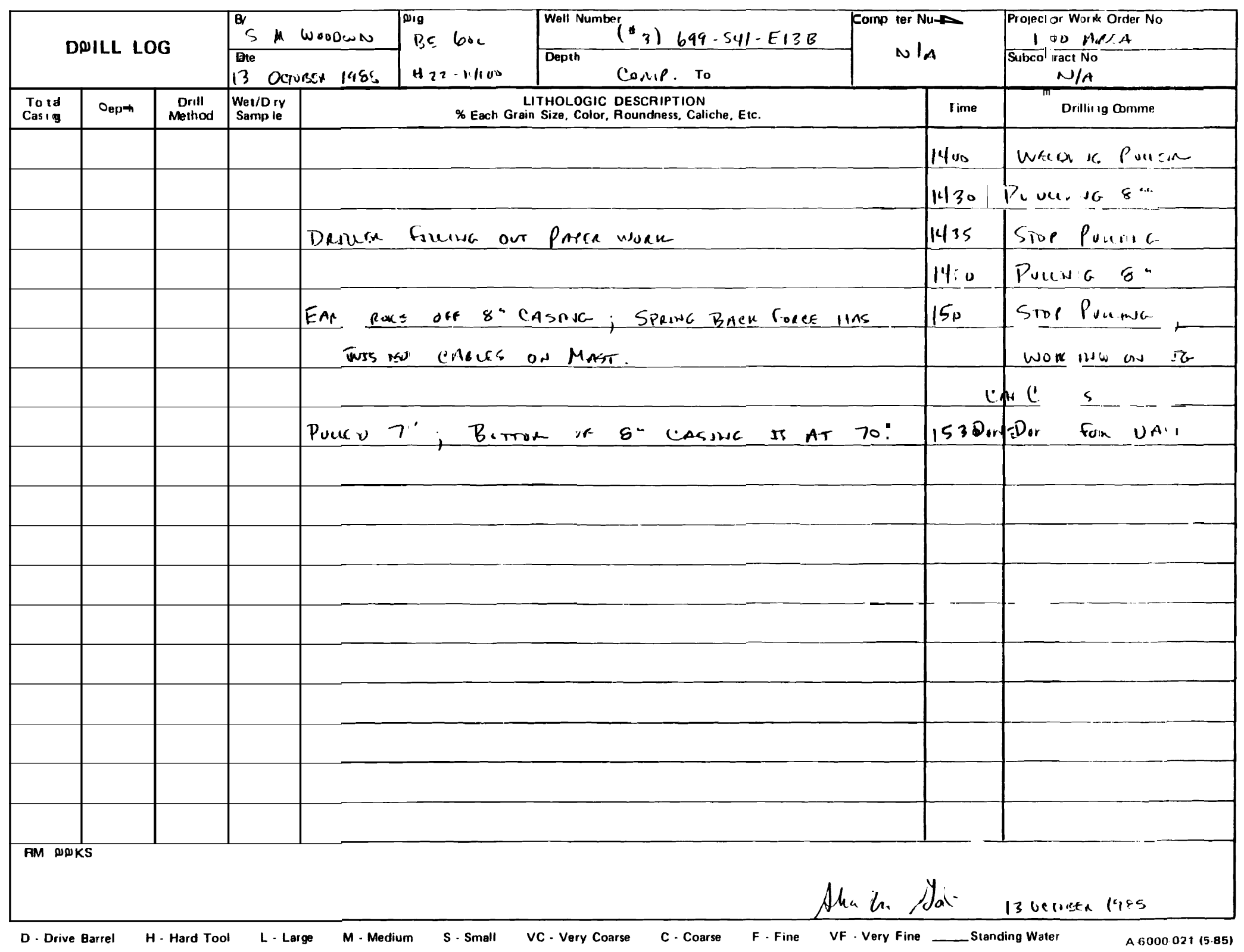


1 of 3

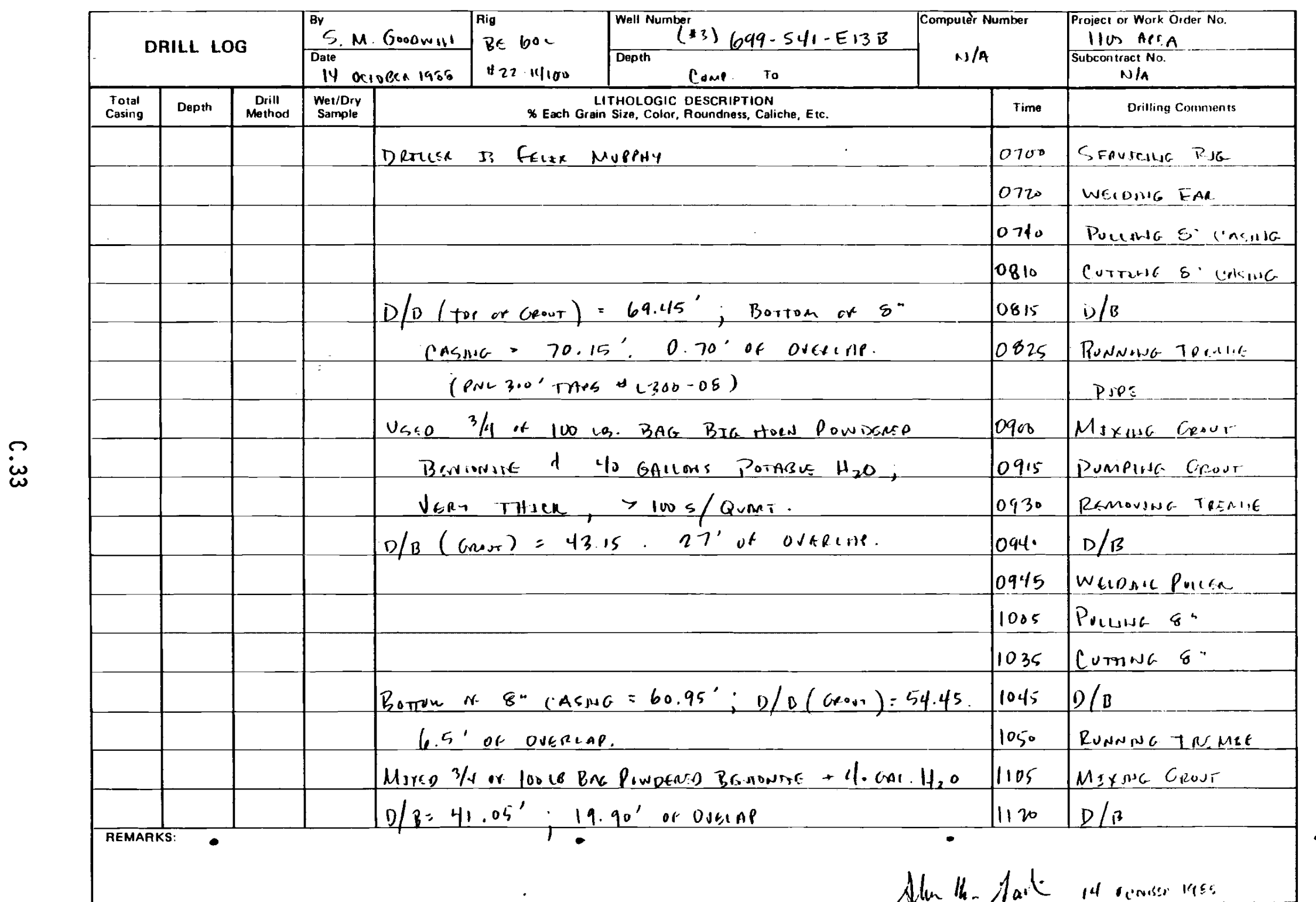

D. Drive Barrel H- Hard Tool L- Large M-Medium S-Small VC-Very Coarse C-Coarse F-Fine VF-Very Fine — Standing Water 


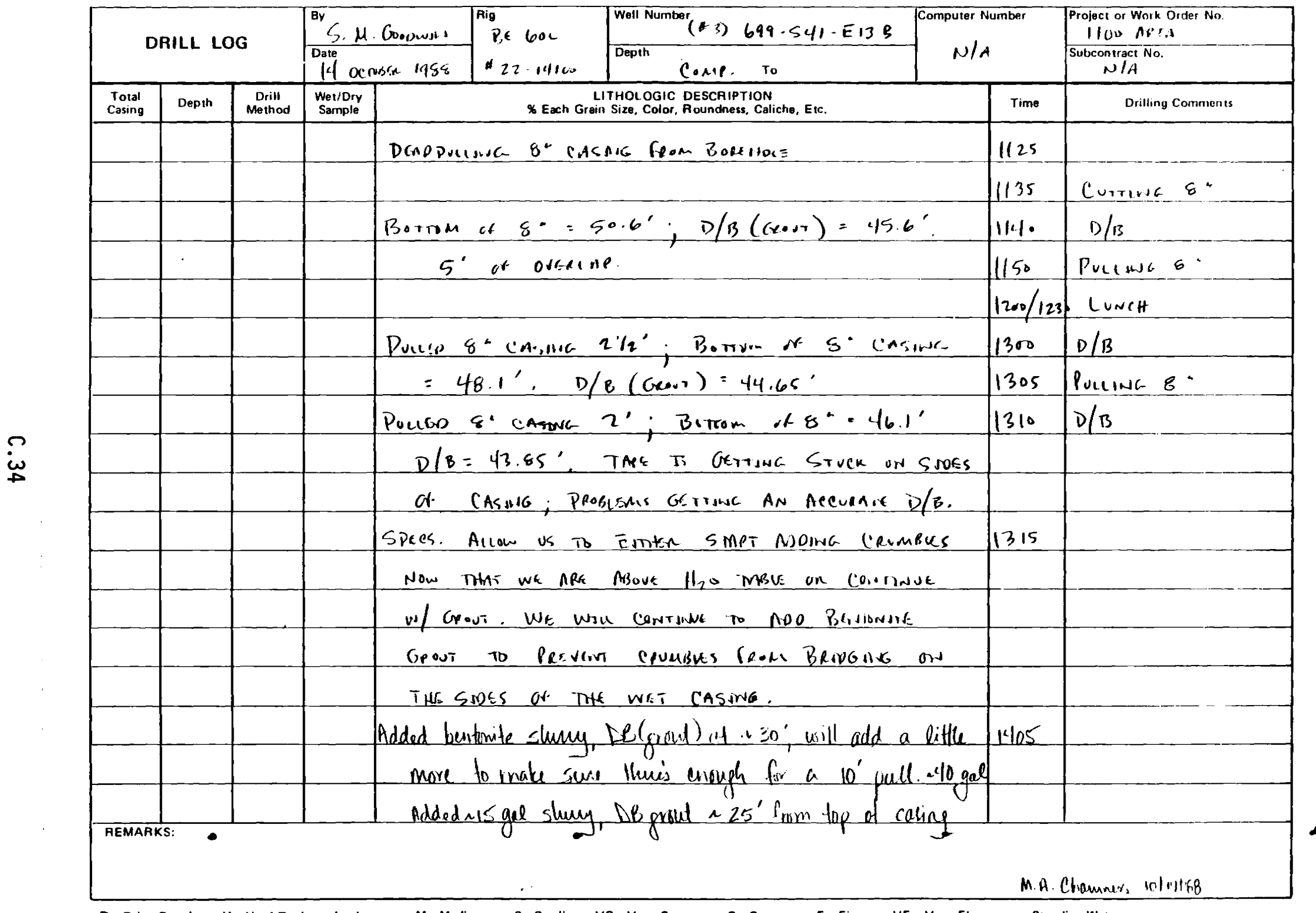

D- Drive Barrel H- Hard Tool L- Large M-Medium S-Small VC - Very Coarse C-Coarse F- Fine VF - Very Fine _- Standing Water A.6000.021 (5.85) 
3 of 3

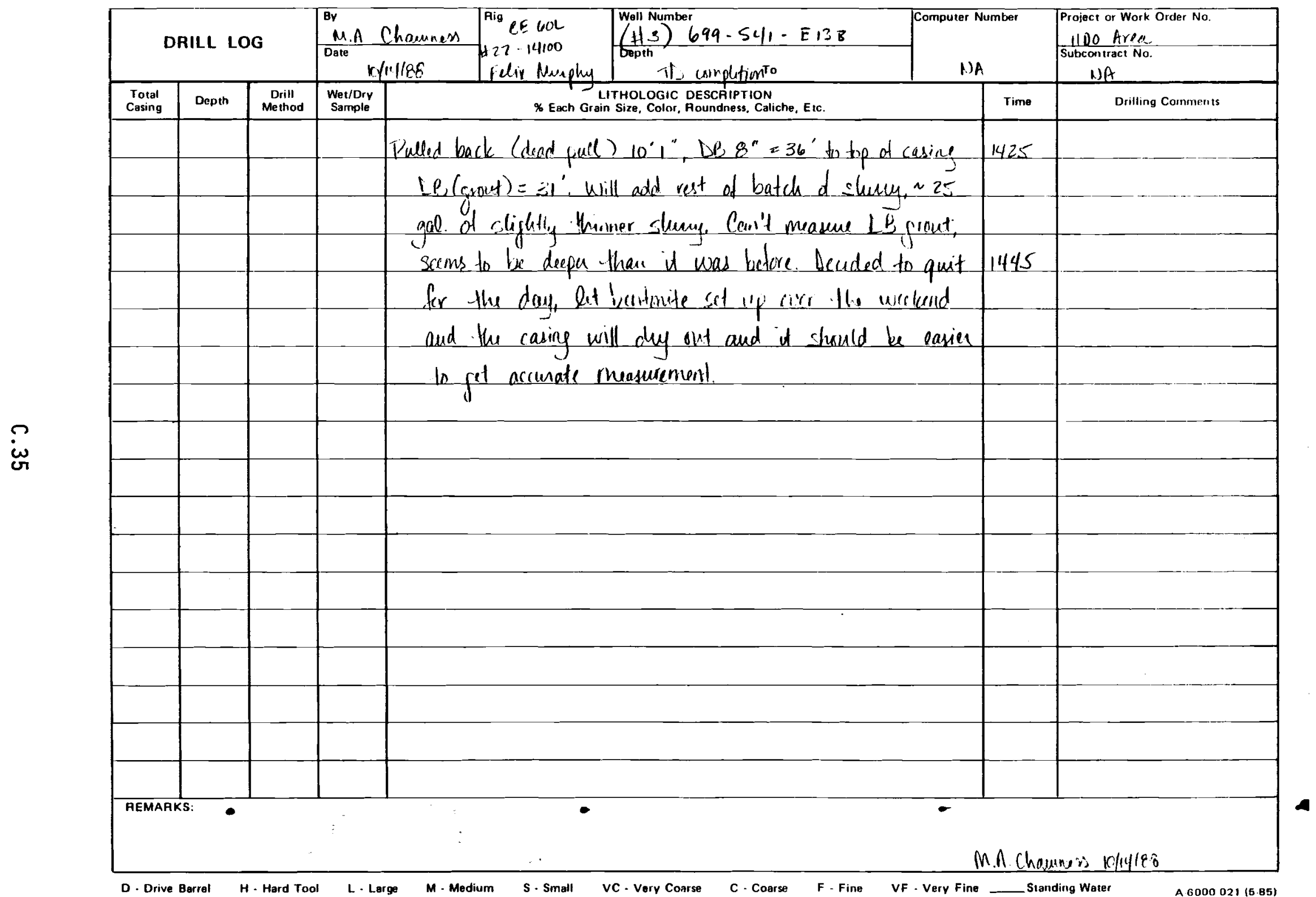




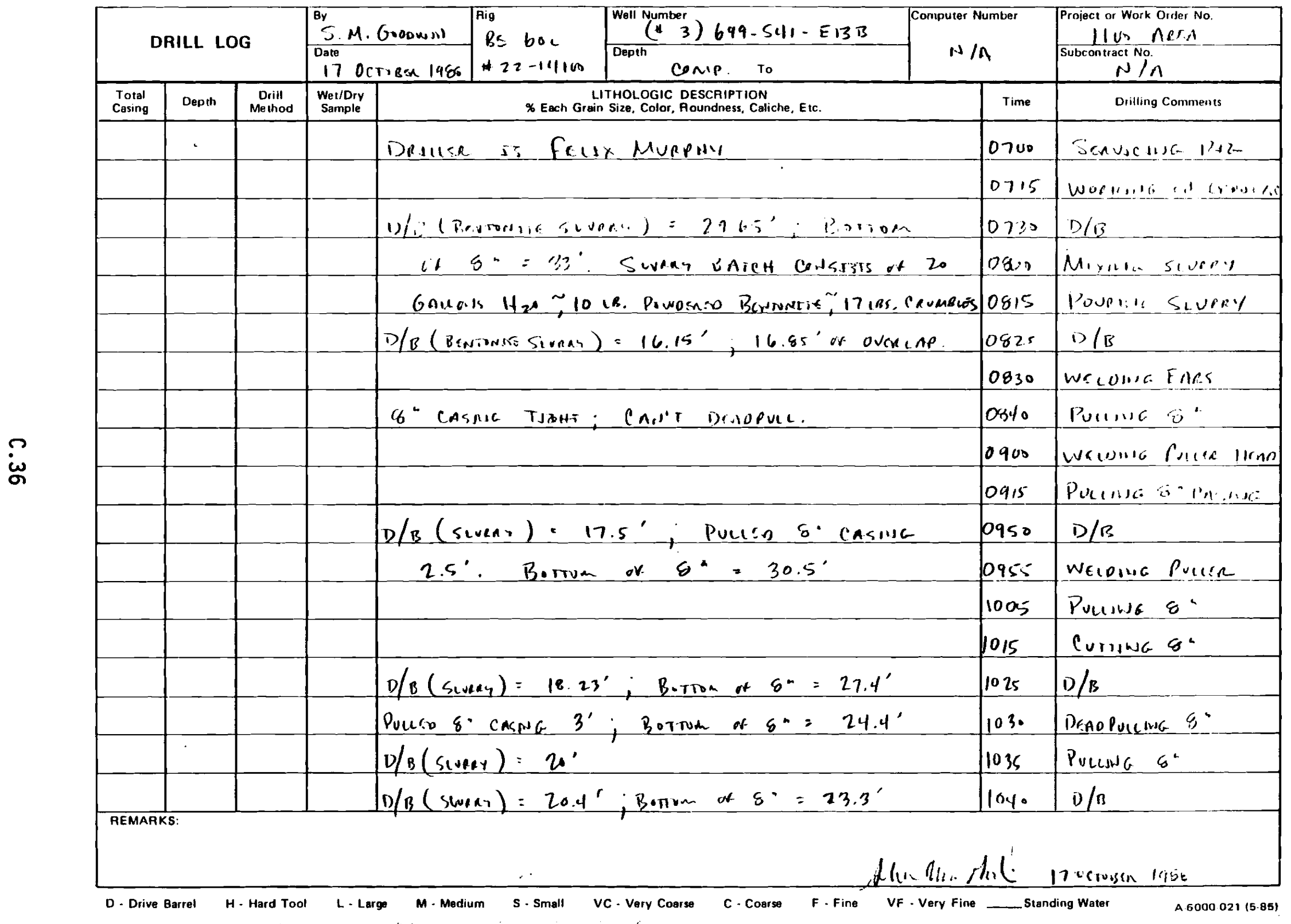


2. of 3

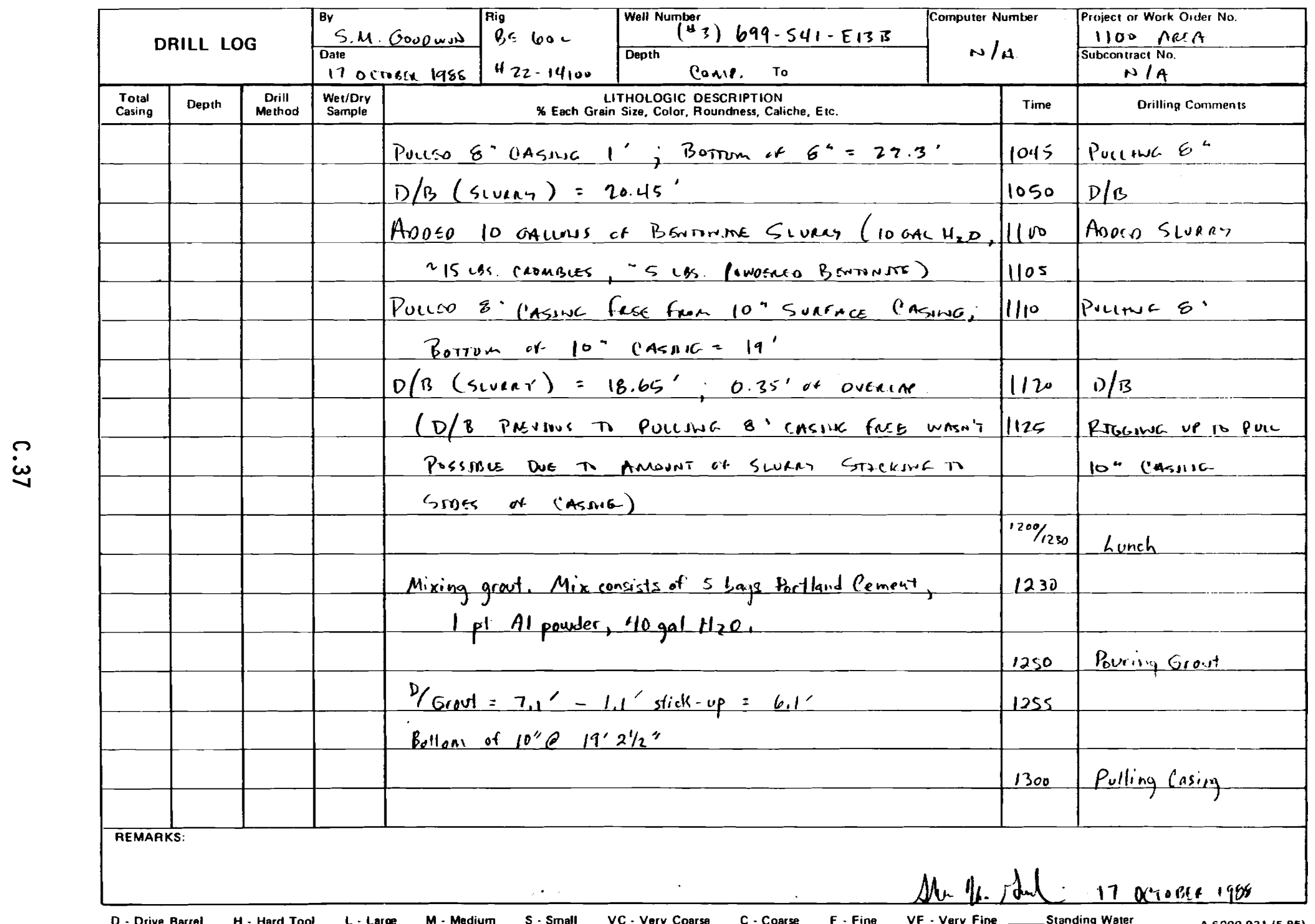




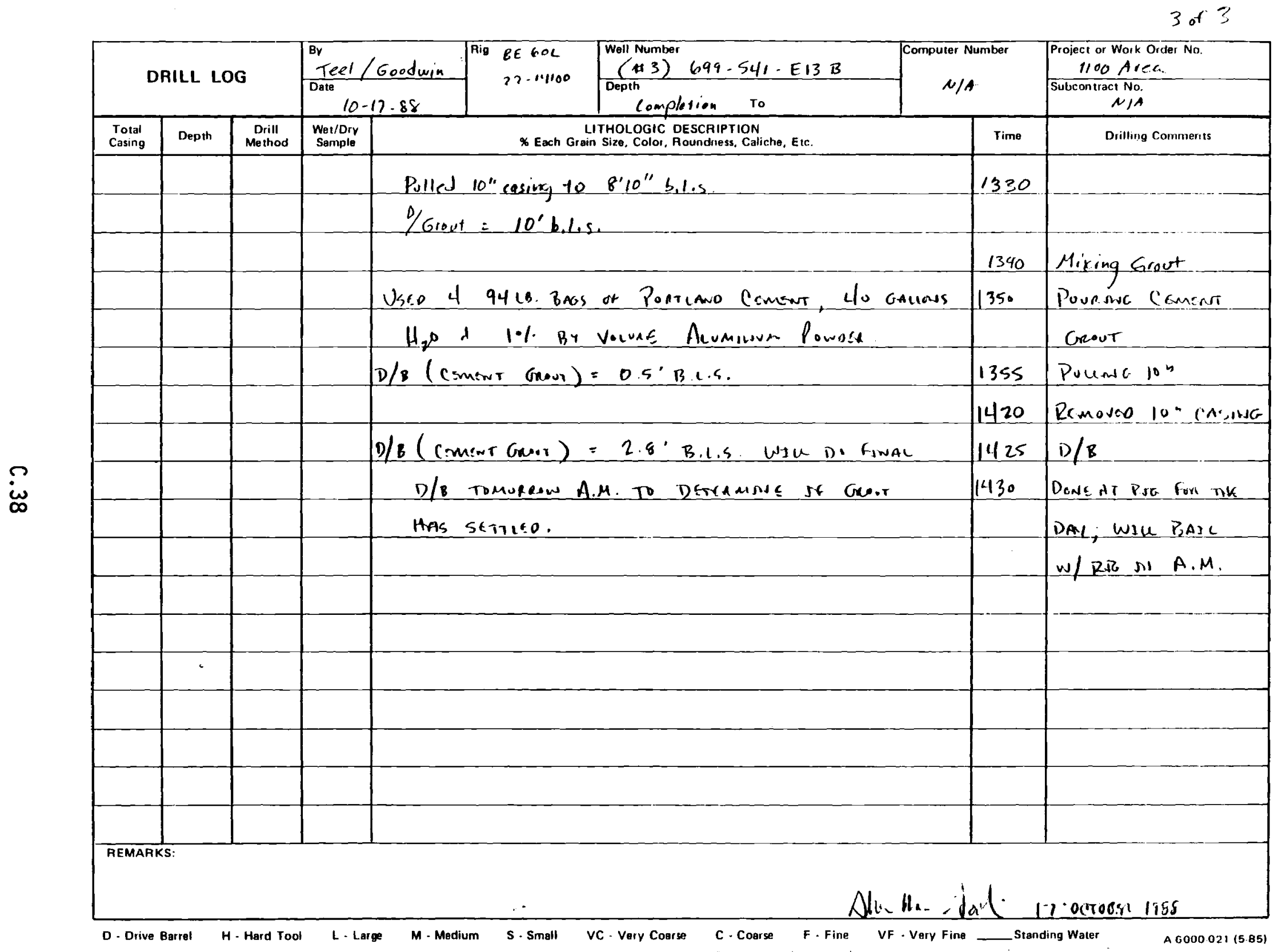




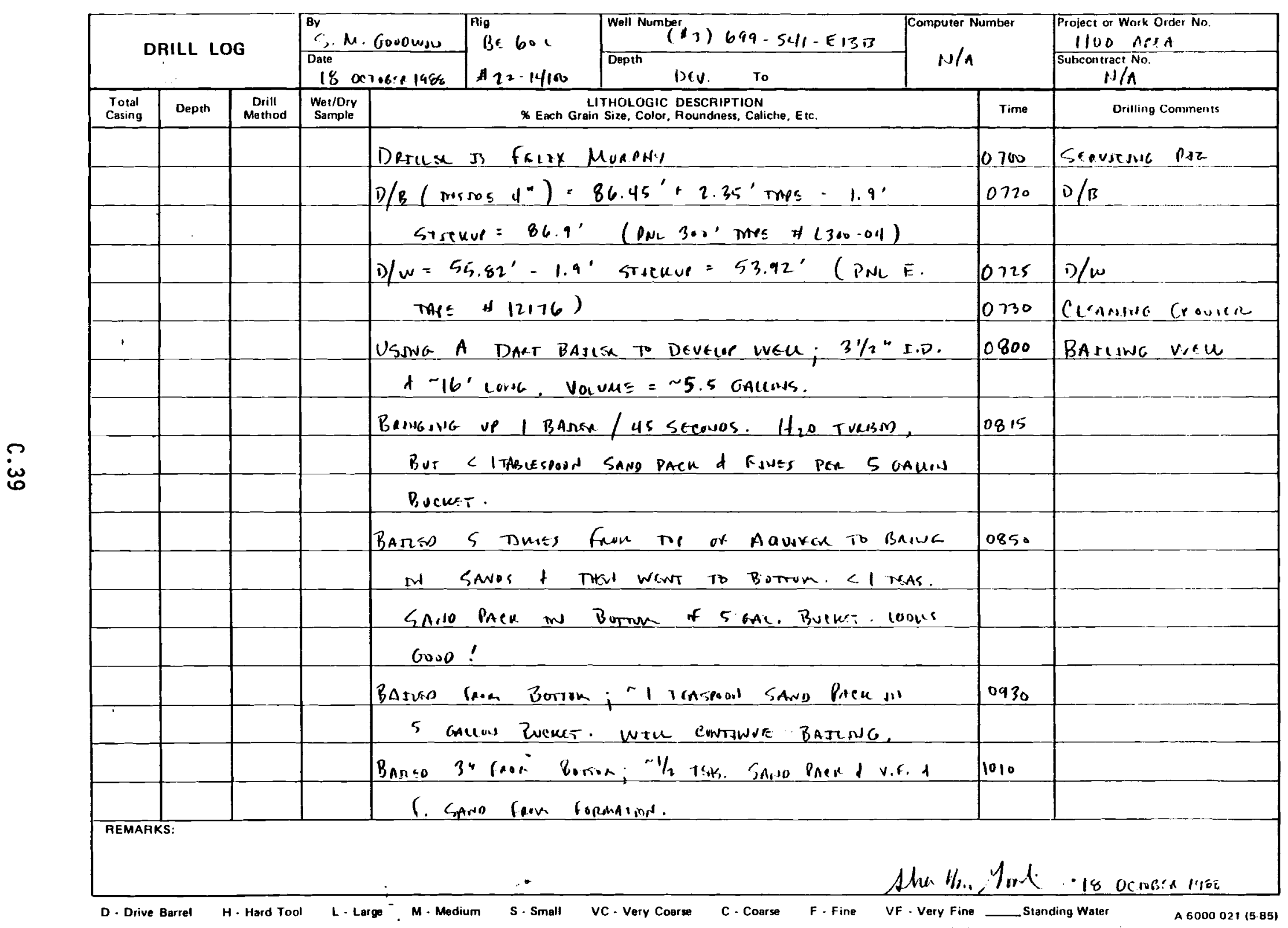




$$
2+2
$$

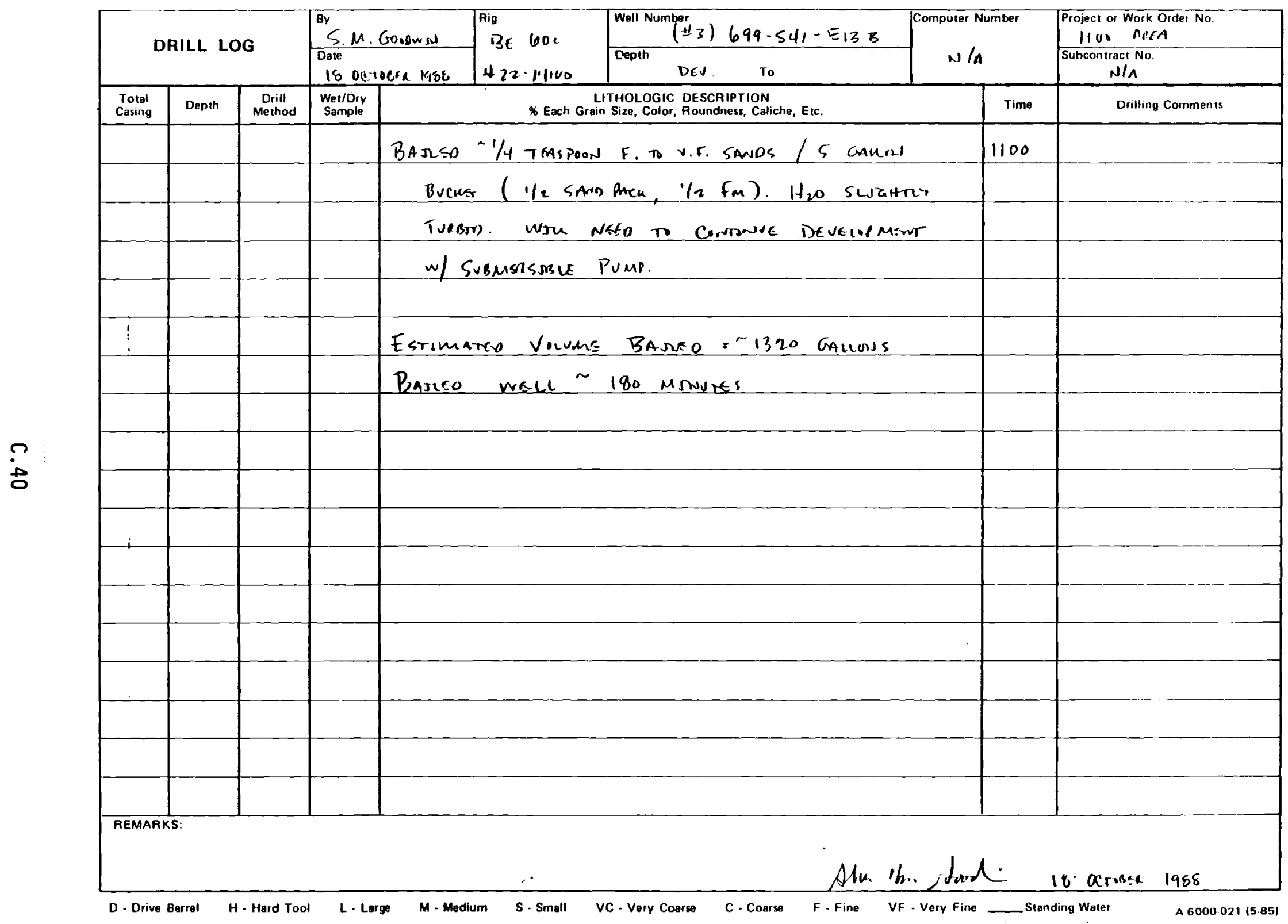




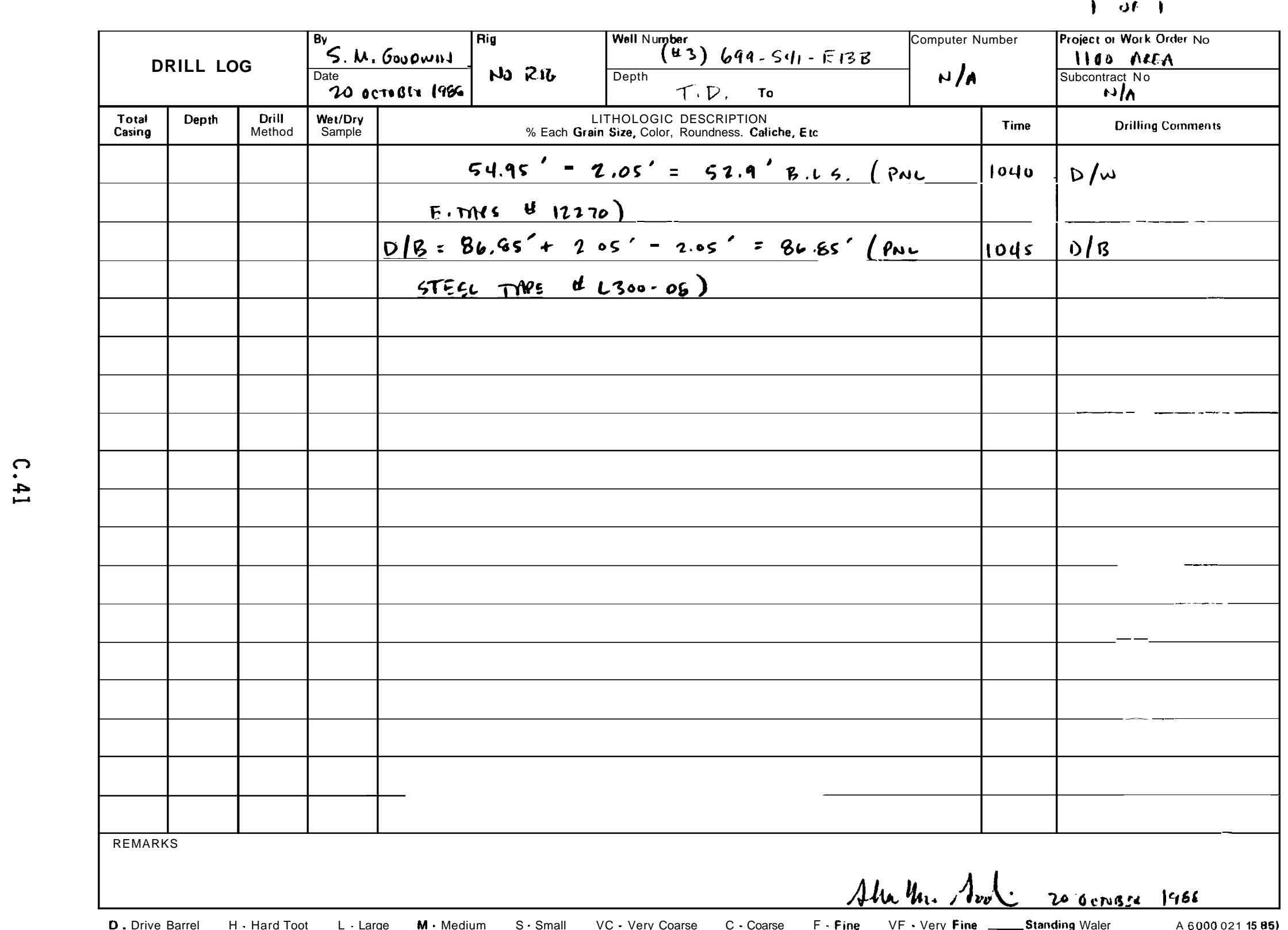




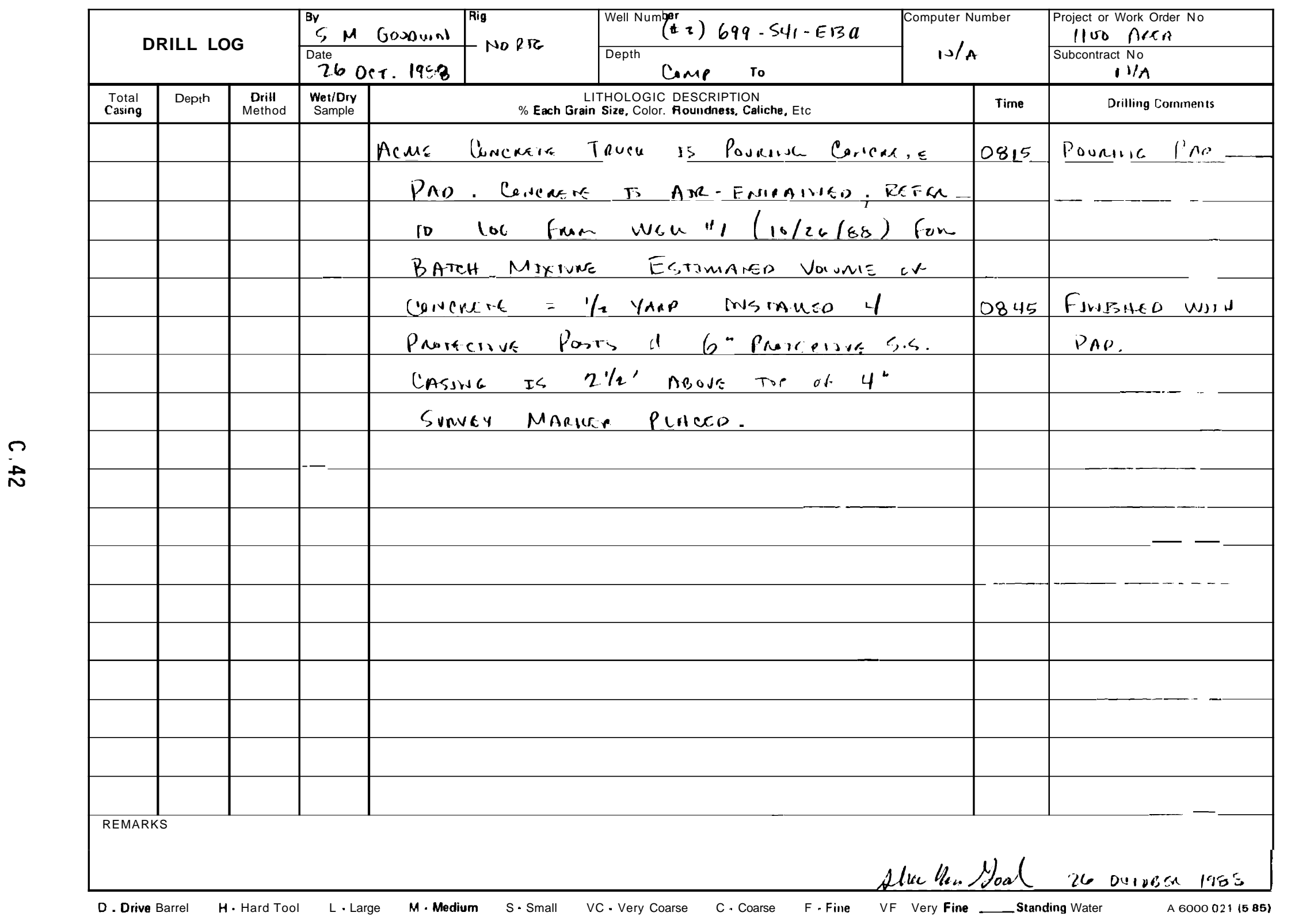


1 if 1

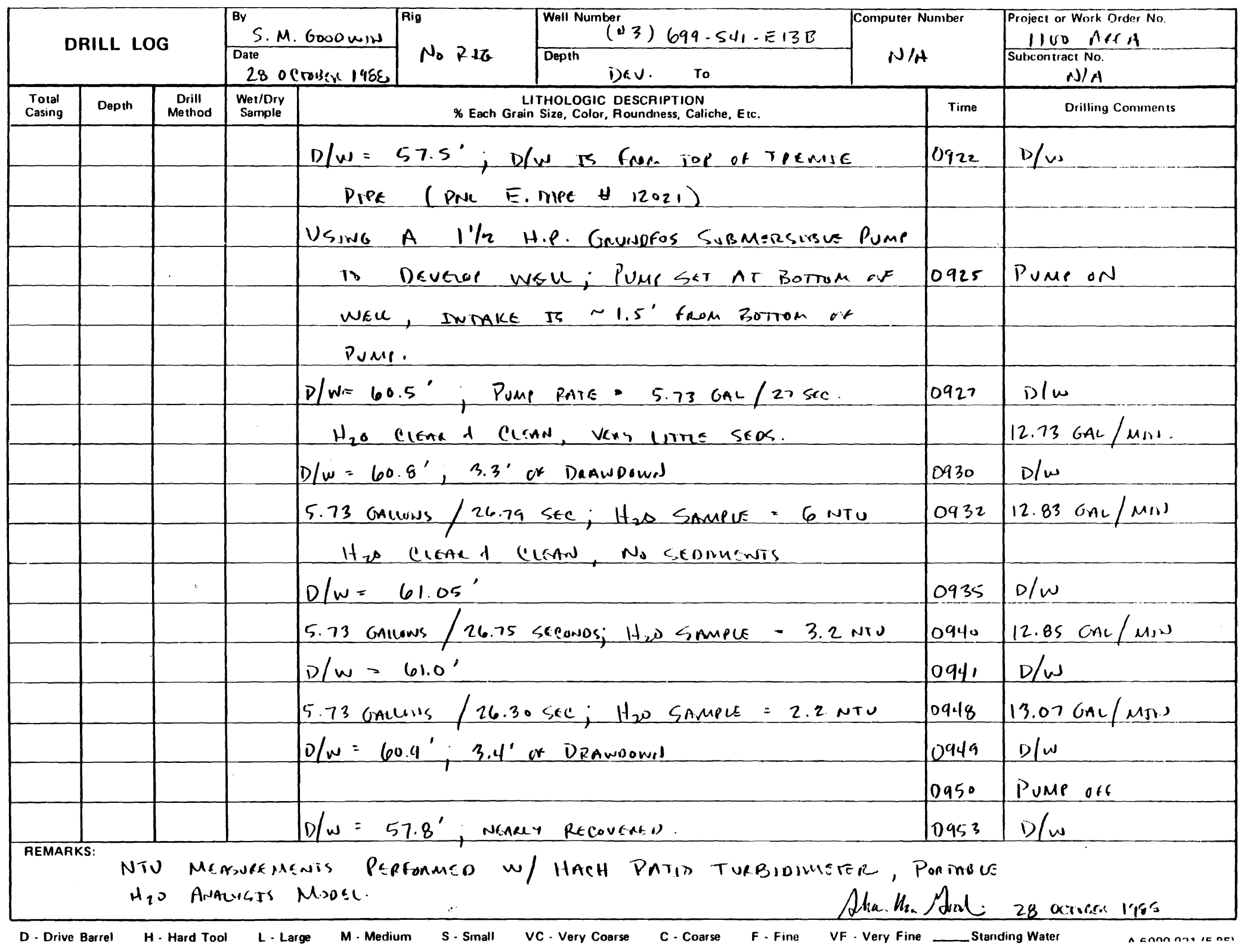


2 of 2

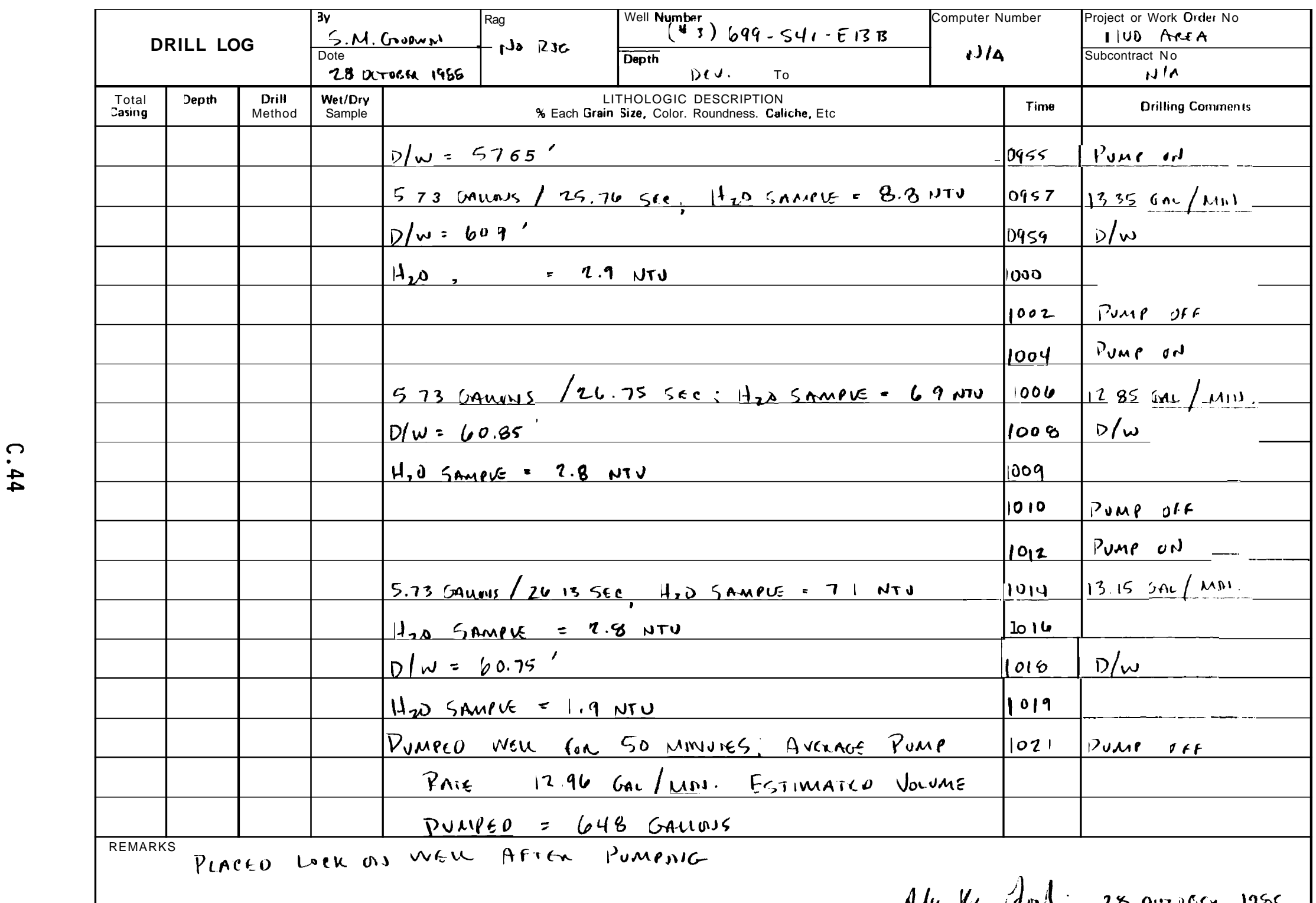

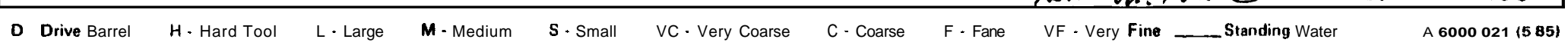


1 of 2

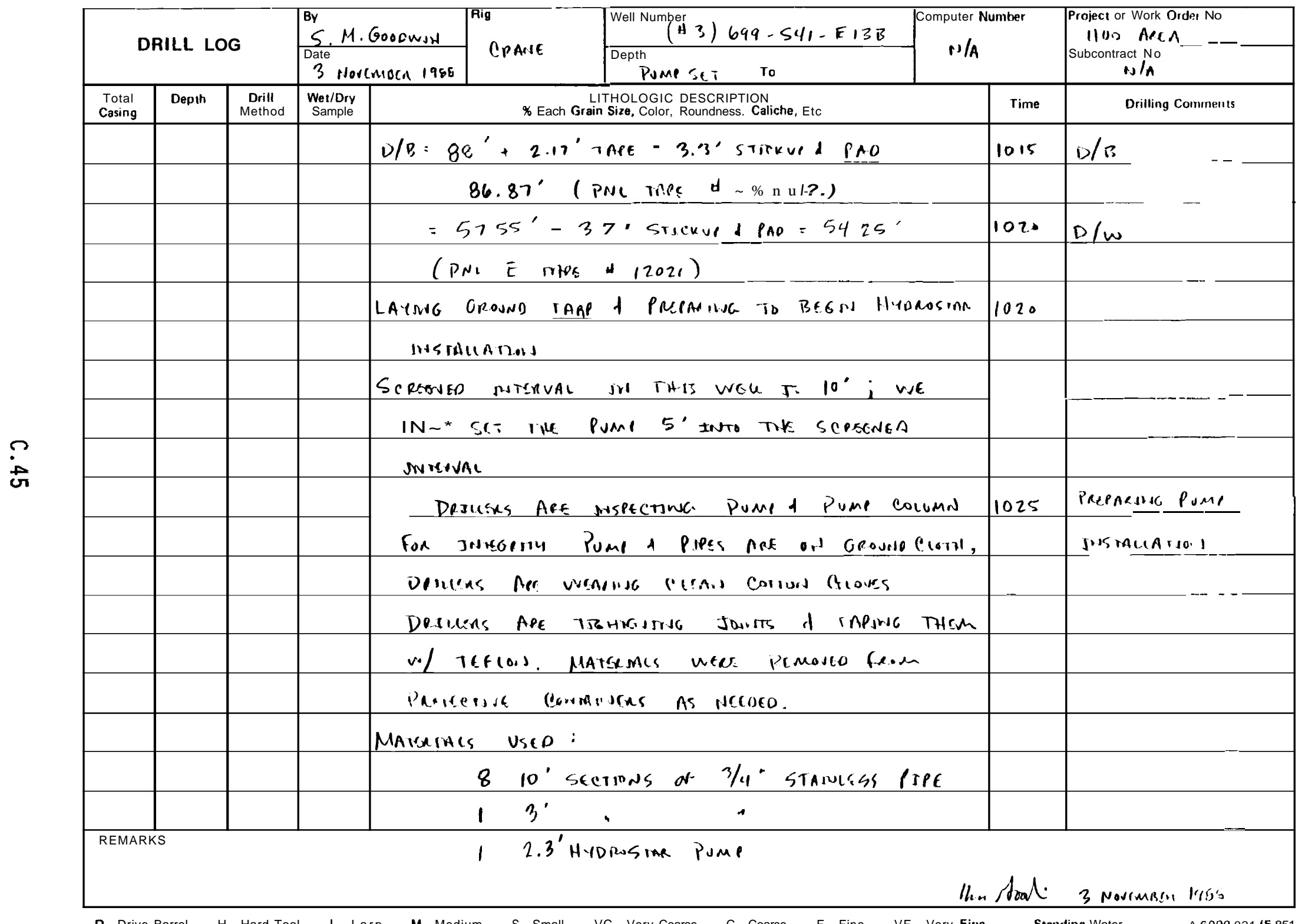


2 of 2

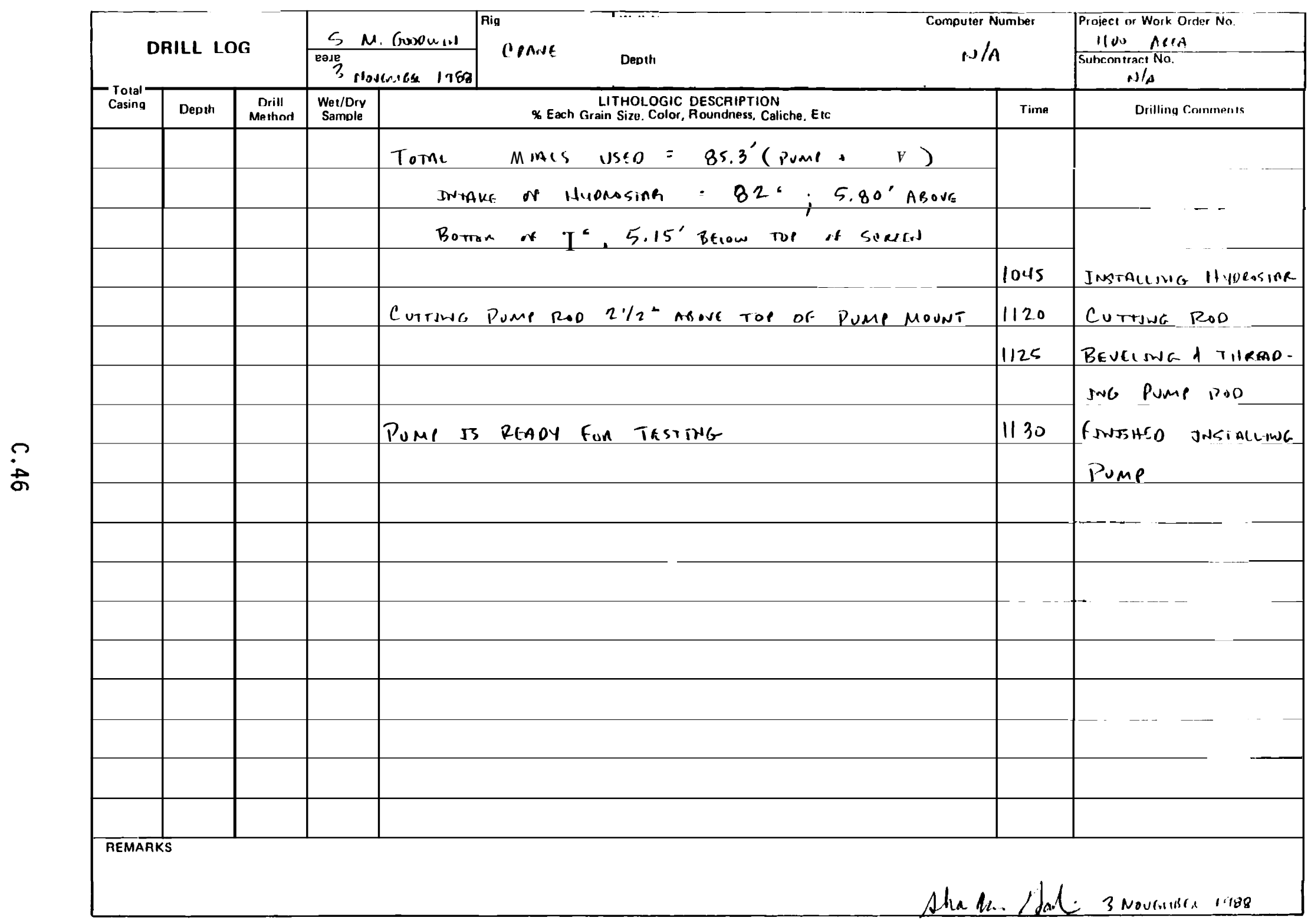

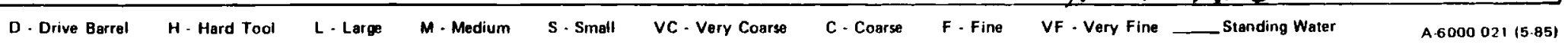




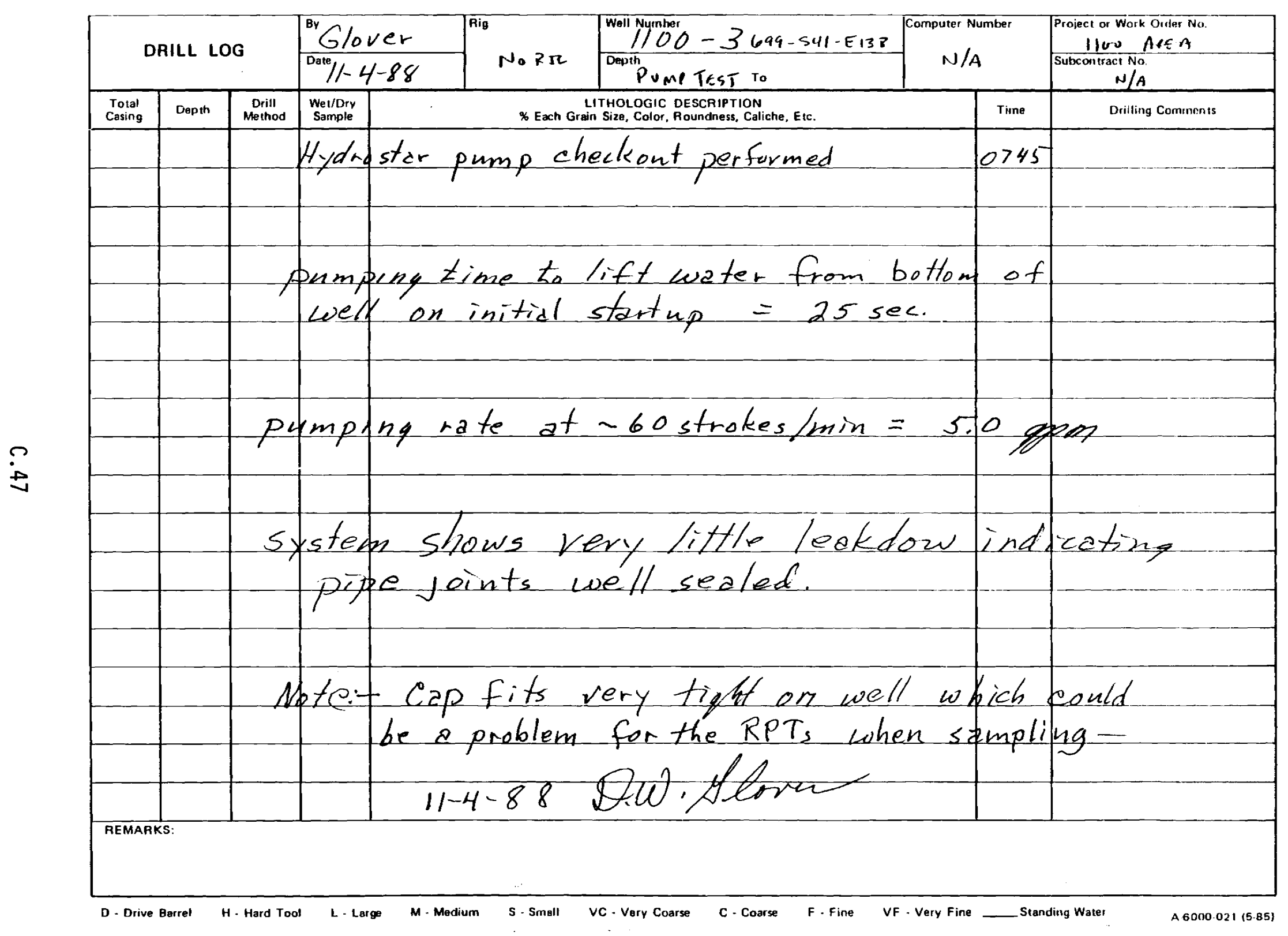


1 of 1

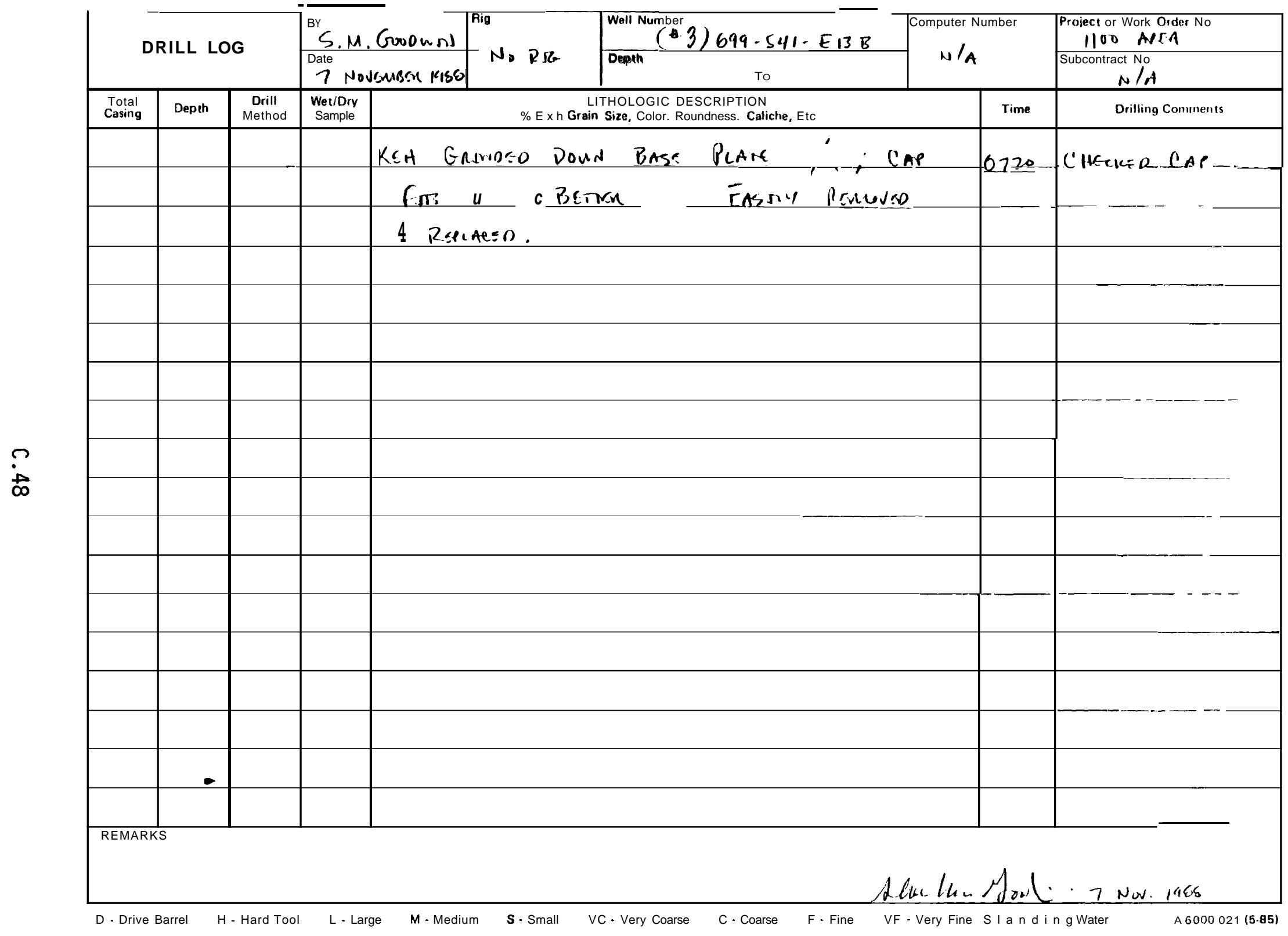




\section{APPENDIX D}

GEOPHYSICAL AND GEOLOGIC LOGS FOR WELL 4 (699-S43-EI2) 


\section{APPENDIX D}

\section{GEOPHYSICAL AND GEOLOGIC LOGS FOR WELL 4 (699-S43-E12)}

This appendix contains the Well Completion/Inspection Report, as-built diagram, notes from the sampling pump installation, the natural gamma log, and the geologists' logs for well 4 (699-S43-E12) in the 1100 Area. 
Procedure P-6

Attachment 1

Page 1 of 1

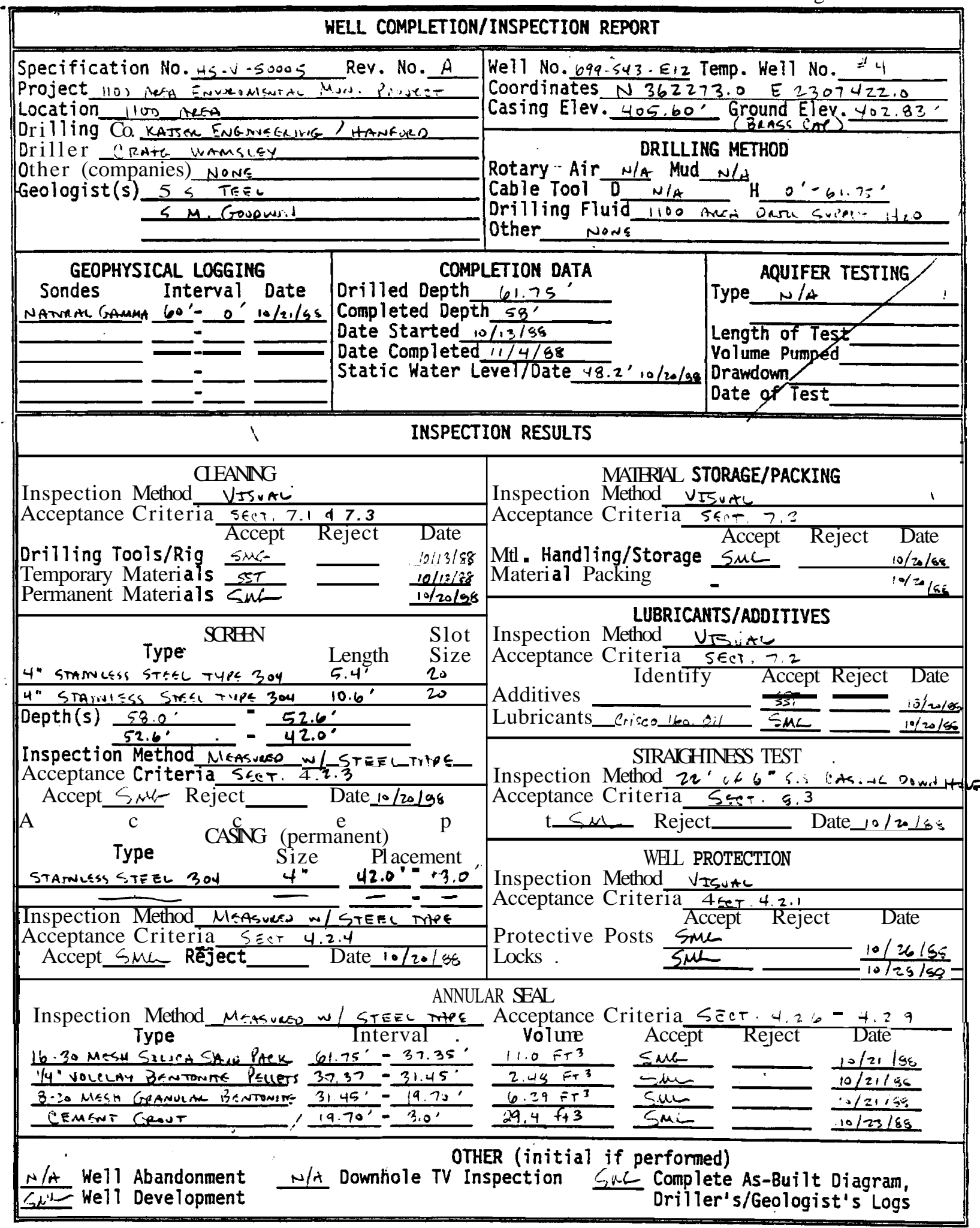

REviewed By 2K. Mncthan 11-8-88 For all blanks mark N/A if not applicable. 


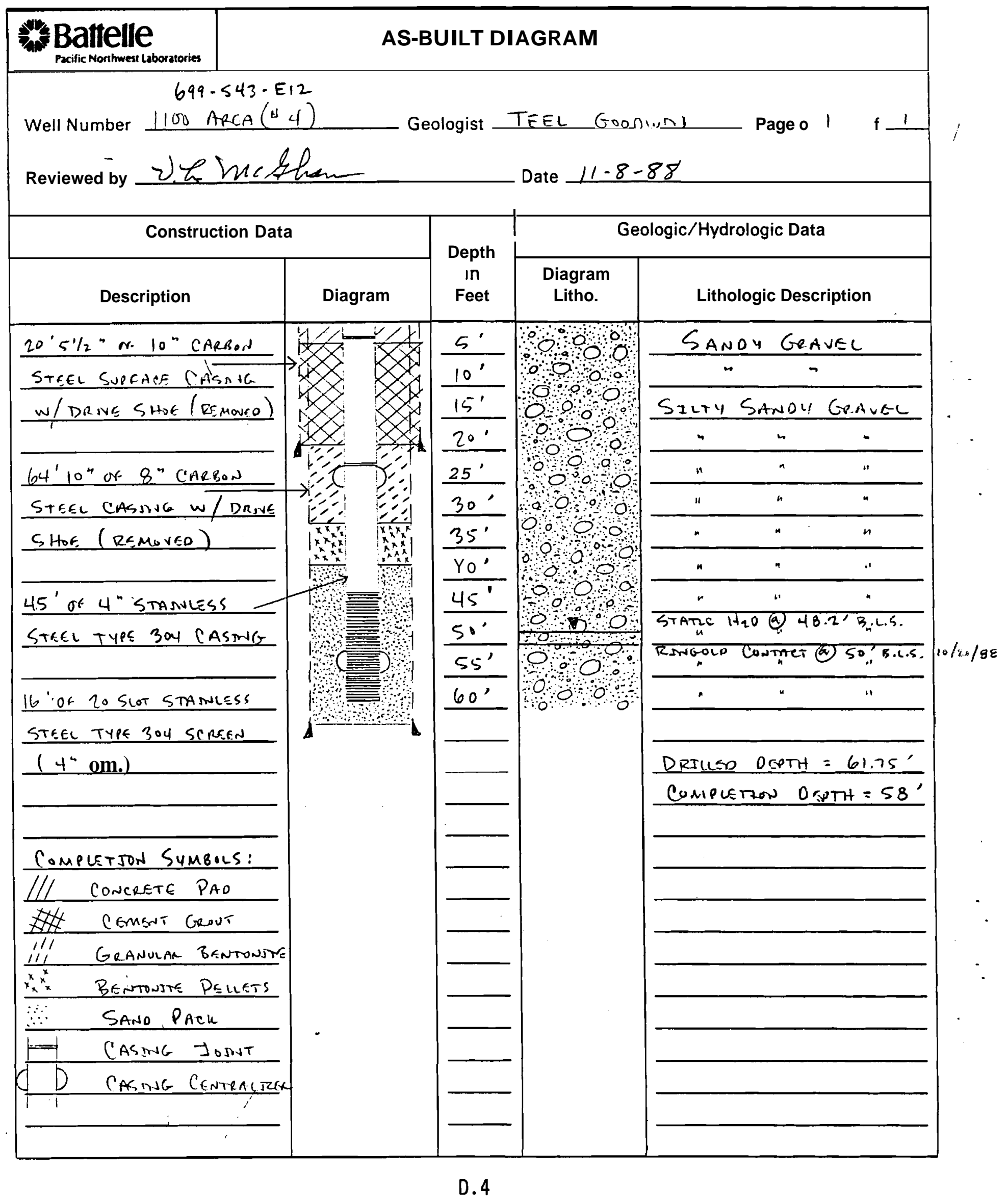


SAMPLING PUMP INSTALLATION IN

GROUND-WATER MONITORING WELLS

$\cdots \quad-\cdots+\cdots \cdots$

Site: $\quad$ Arfat

Monitoring Well Number: $(44) 699-543-E_{12}$

Depth to Water: $49.95^{\circ}$

Depth to Bottom: $57.92^{\circ}$ Reported Depth to Bottom: $58^{\circ}$

Pump Type: Positive Displacement Piston

Pump Model : Hydrostar HS-8001

Date Installed: $11 / 4 / 89$

Installed By: KEH: OLIN AMOS CRAIG WAMSLSY. LENNY COROON

Pump Discharge Pipe Description:

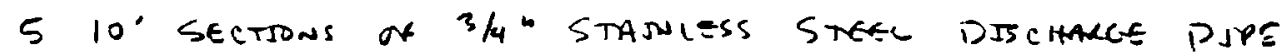

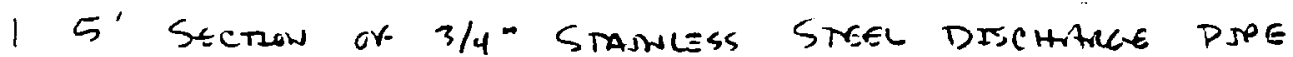

$5^{\prime}$ Sections Is Betweas $10^{\circ} \mathrm{d} 15^{\circ}$ Bslow Pump Bass plate.

Additional Comments:

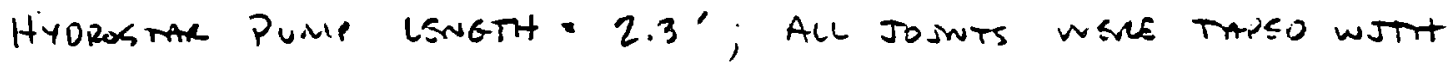
TEFLON. DRJULAS WORE CLSAN COTTON GLOVES.

Sketch of Pump Installation:

Completed By:_Shanth__food._Date: $11 / 4 / 55$ 


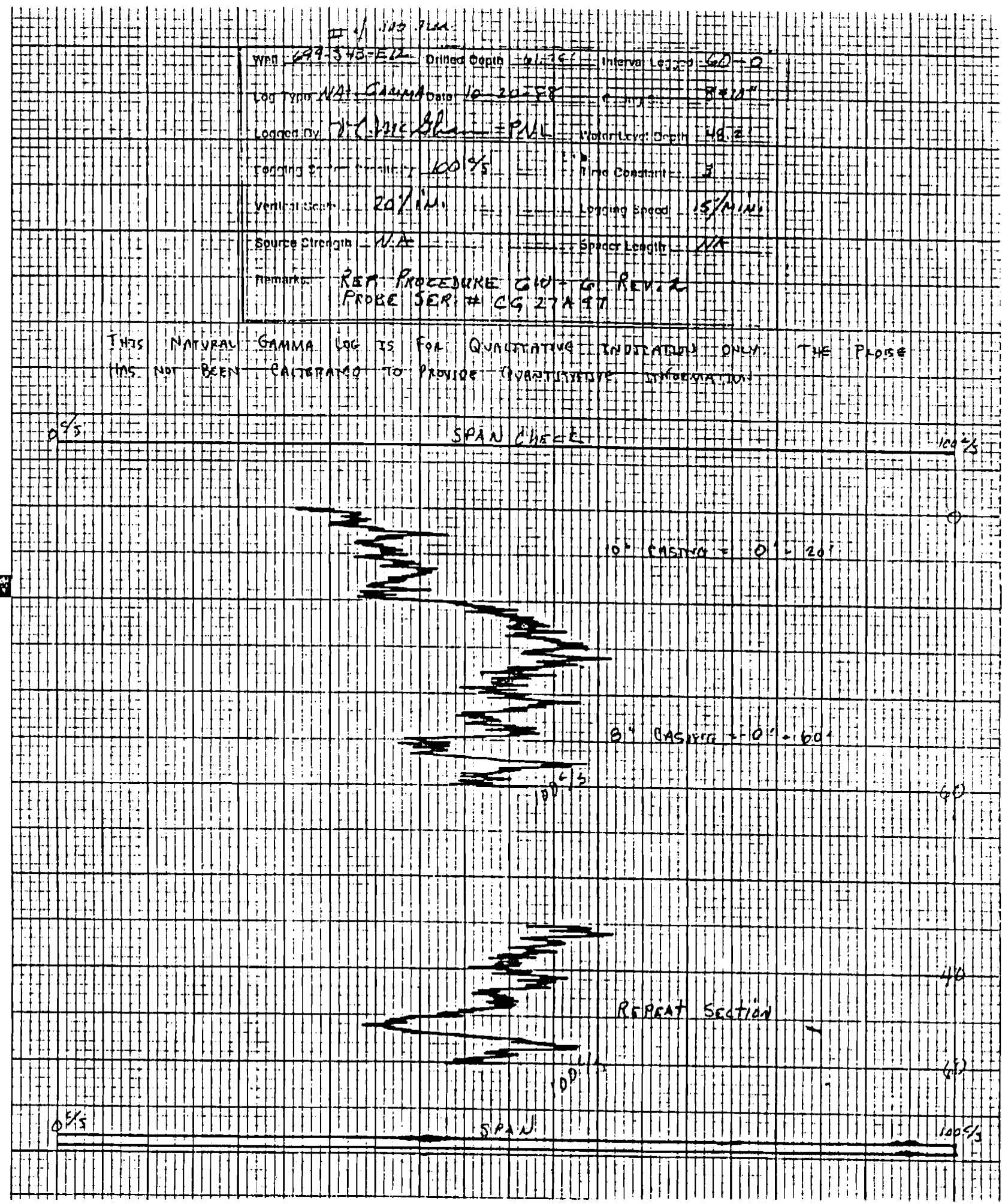


1.11

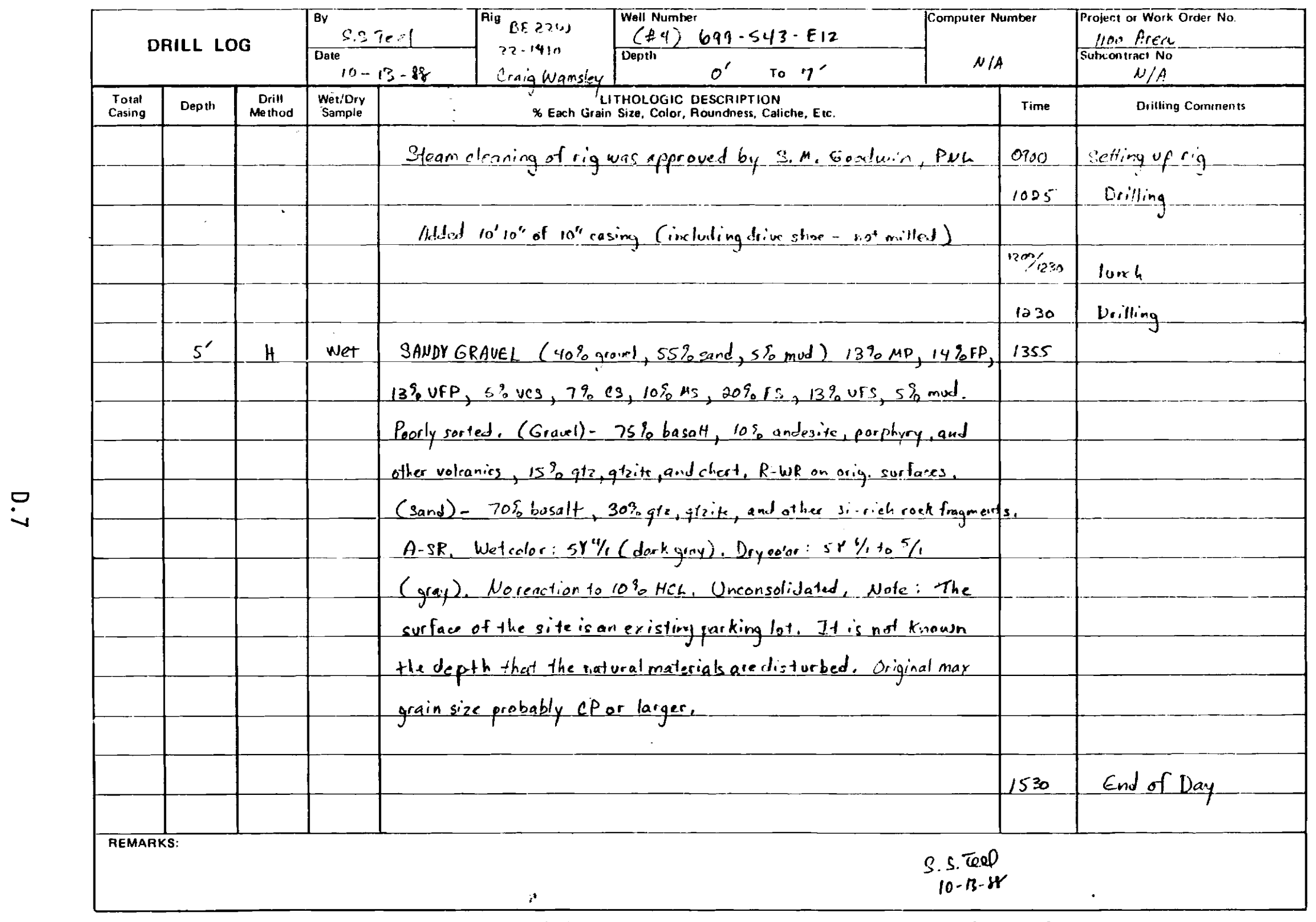

D. Drive Barrel H-Hard Tool L-Large M-Medium S-Small VC-Very Coarse C - Coarse F-Fine VF - Very Fine - Standing Water 
$r_{1}, \mathrm{r}$

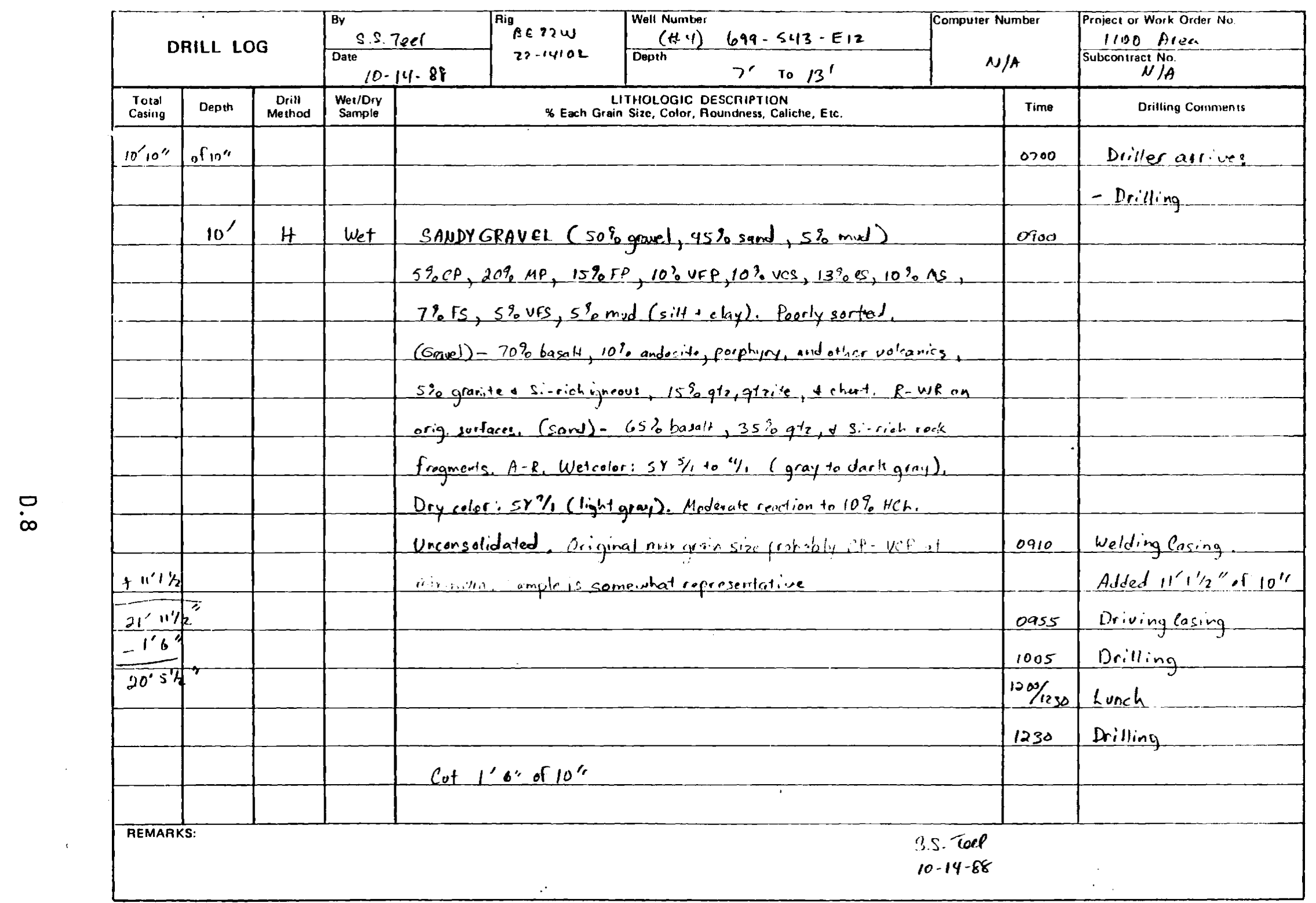

D-Drive Barrel H- Hard Tool L-Large M-Medium S-Small VC-Very Coerse C-Coarse F-Fine VF-Very Fine _-Standing Water , 
a of 3

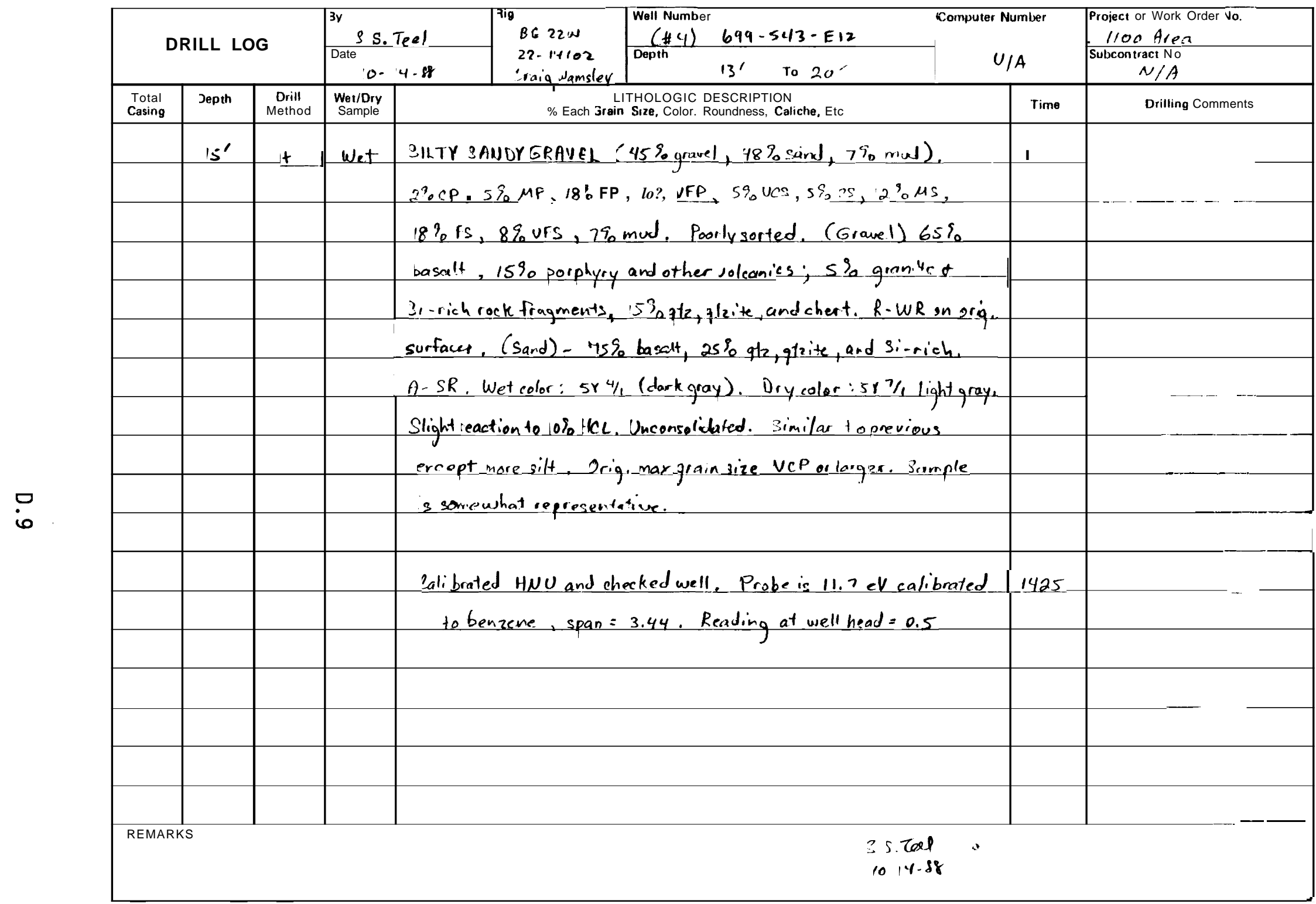

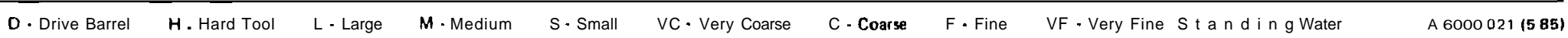




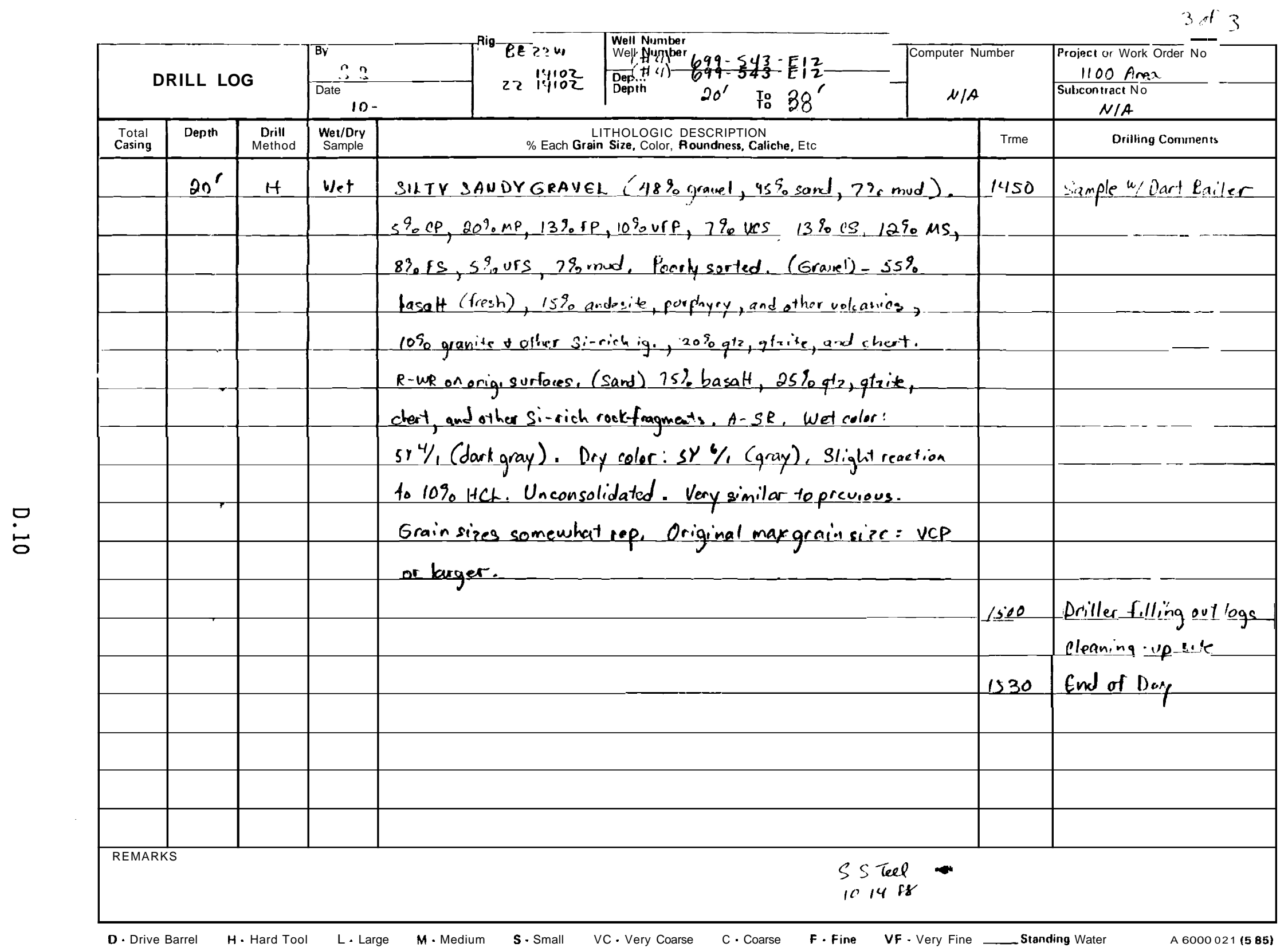


1.12

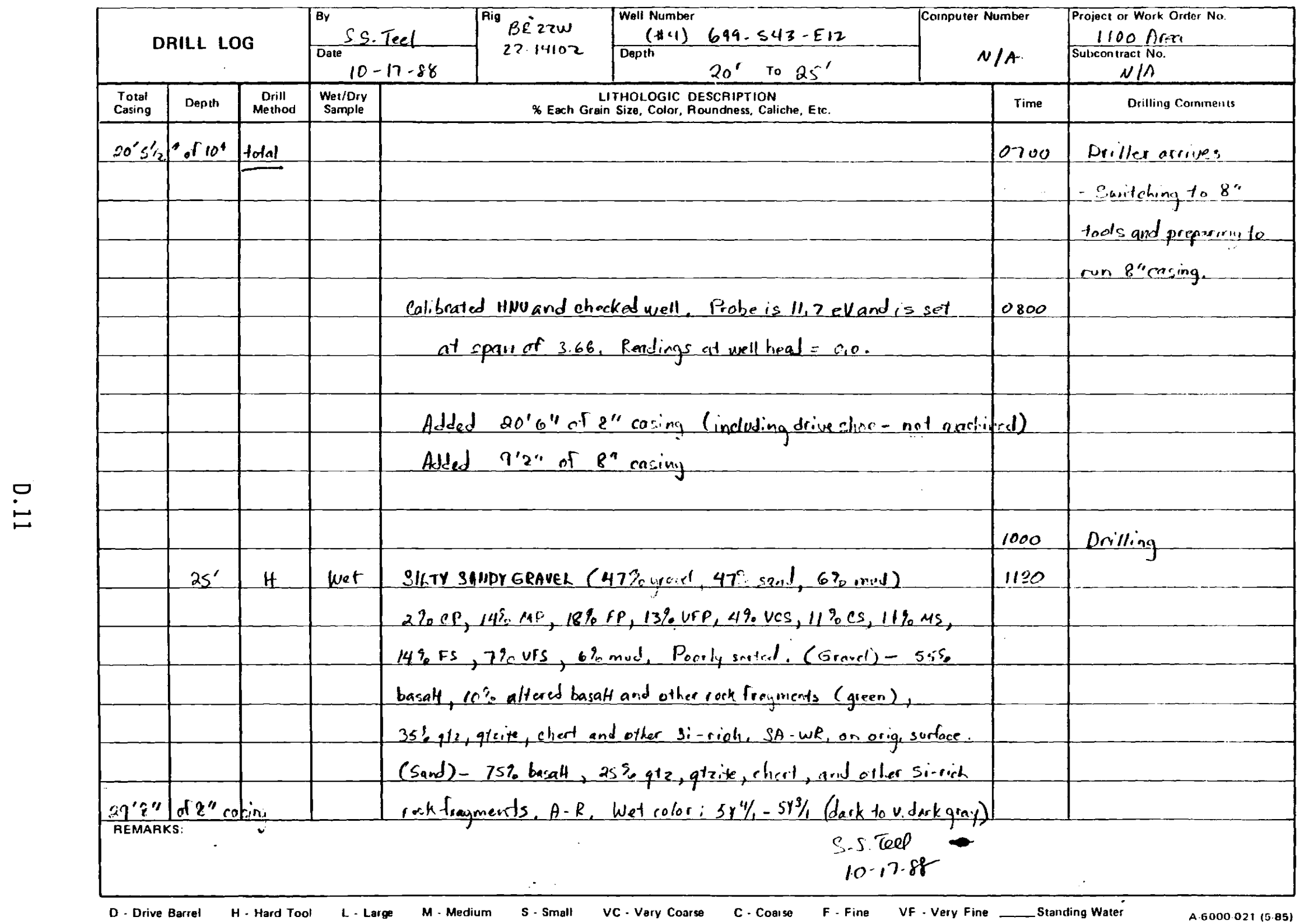


2 of a

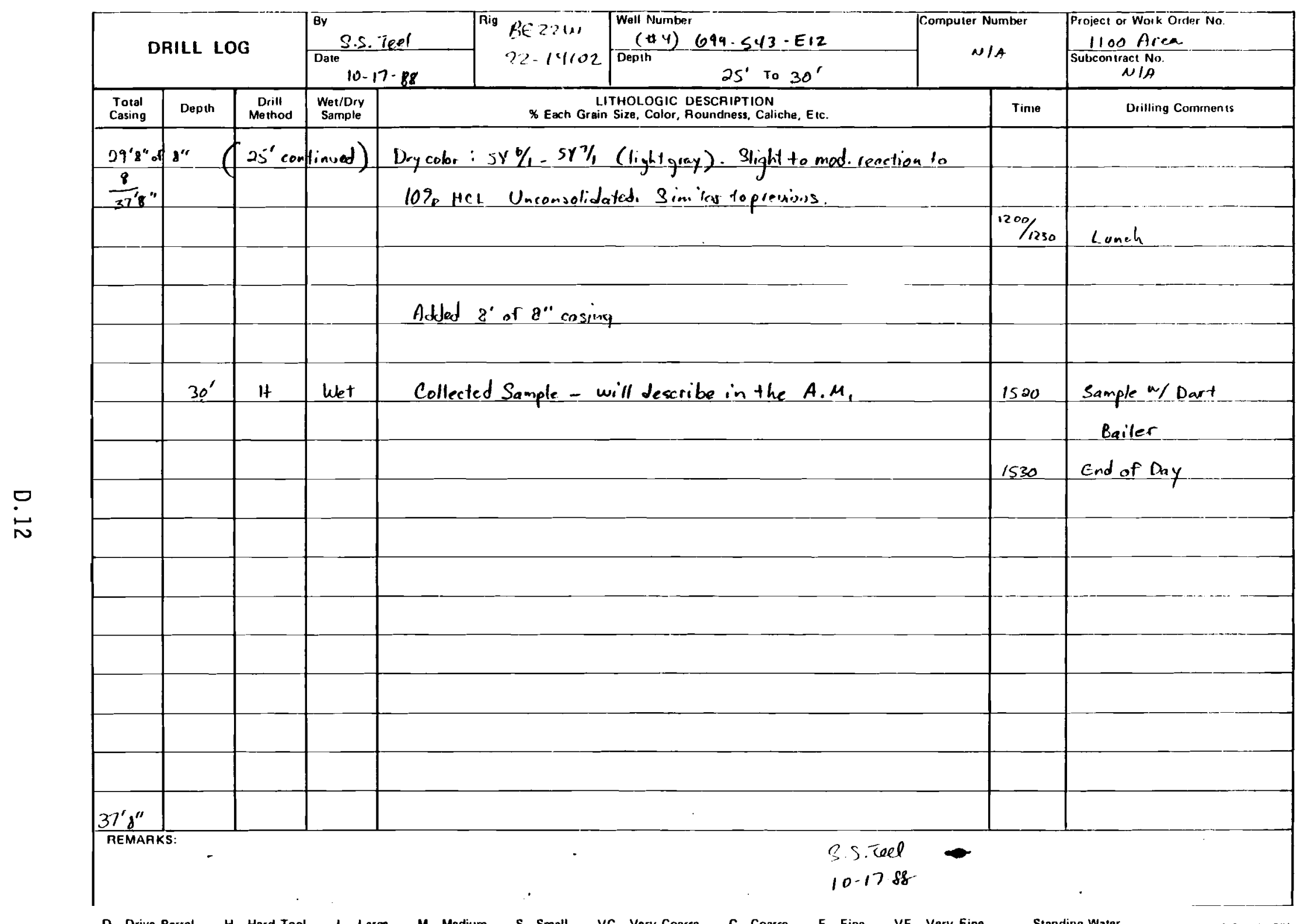


lof 4

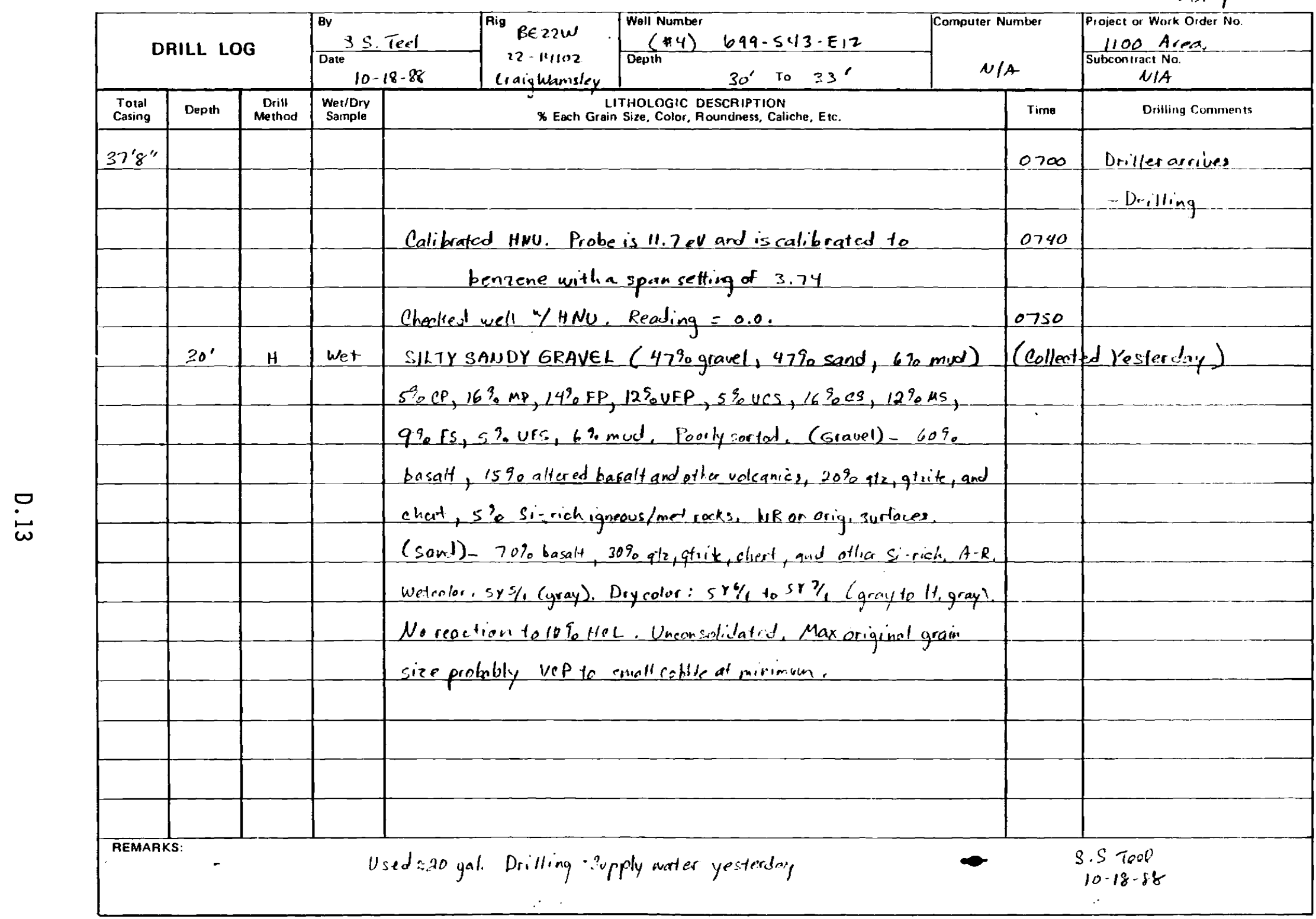

D. Drive Barrel H. Hard Tool L- Large M-Medium S-Small VC-Very Coarse C-Coarse F-Fine VF - Very Fine _- Standing Water A.6000 021 (5.85) 
2 if 4

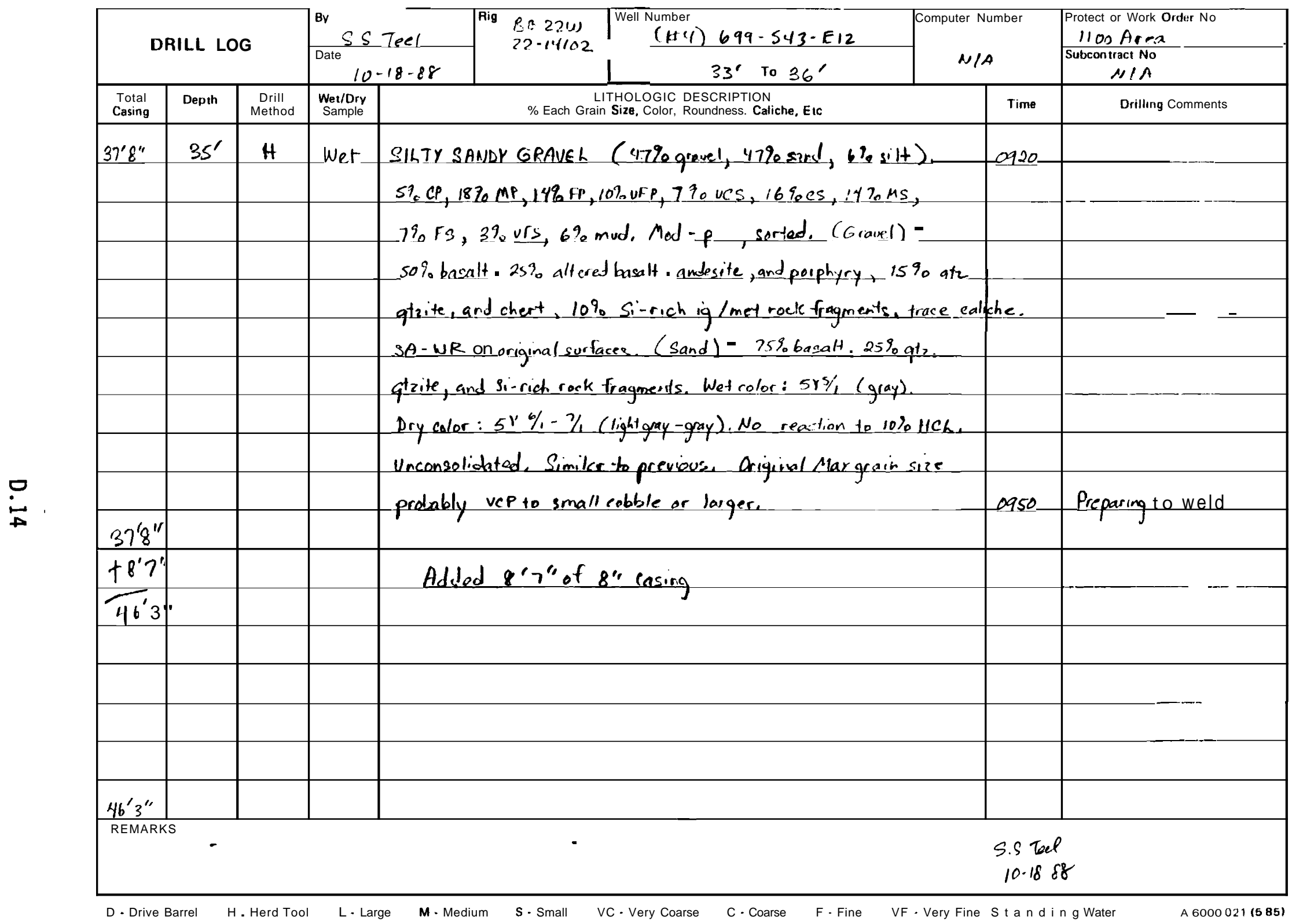


$3 .+4$

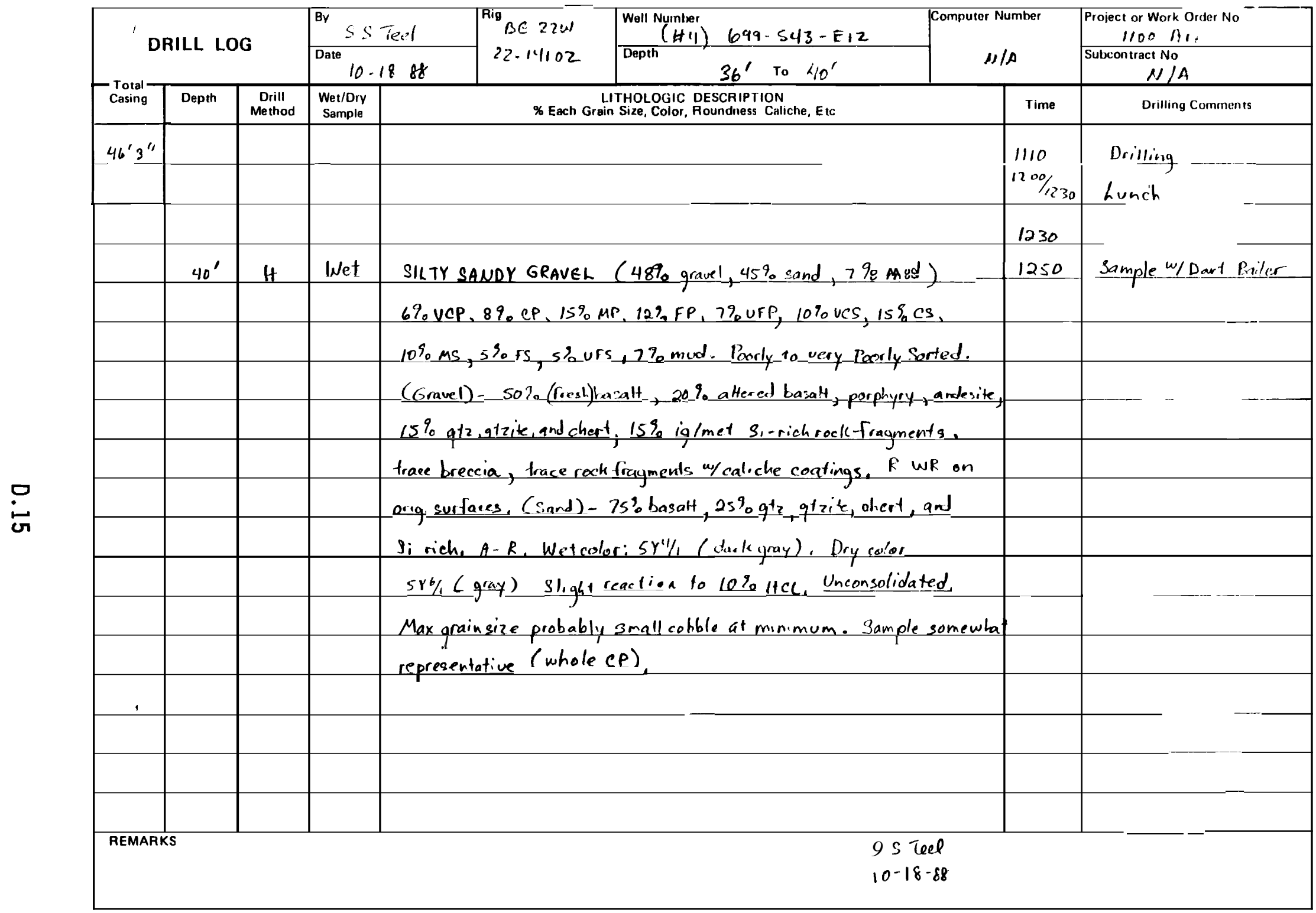

D - Drive Barrel H. Hard Tool L- Large M-Medium S-Small VC - Very Coarse $\quad$ C - Coarse F- Fine VF - Very Fine _- Standing Water 
4 or 4

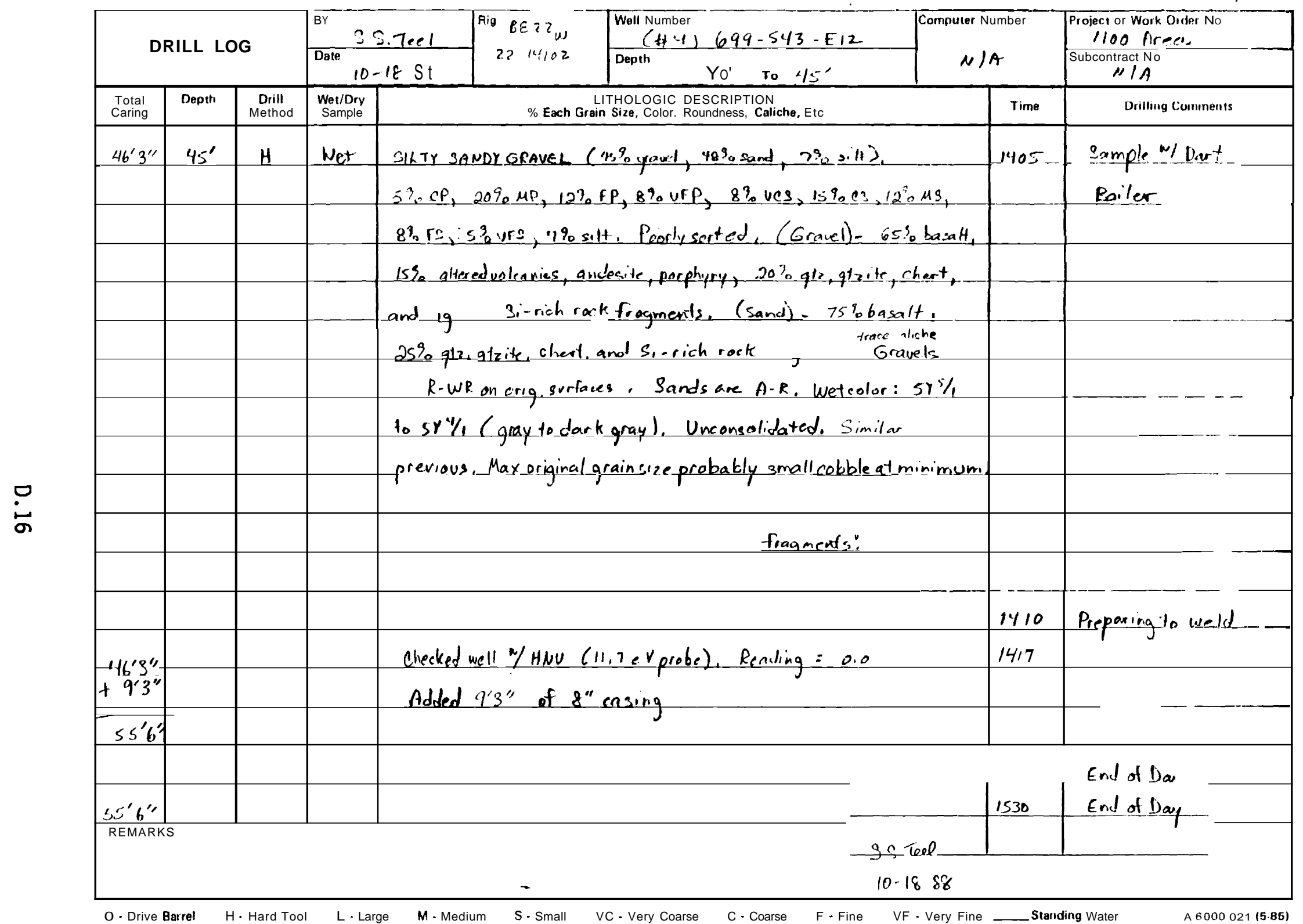


lof

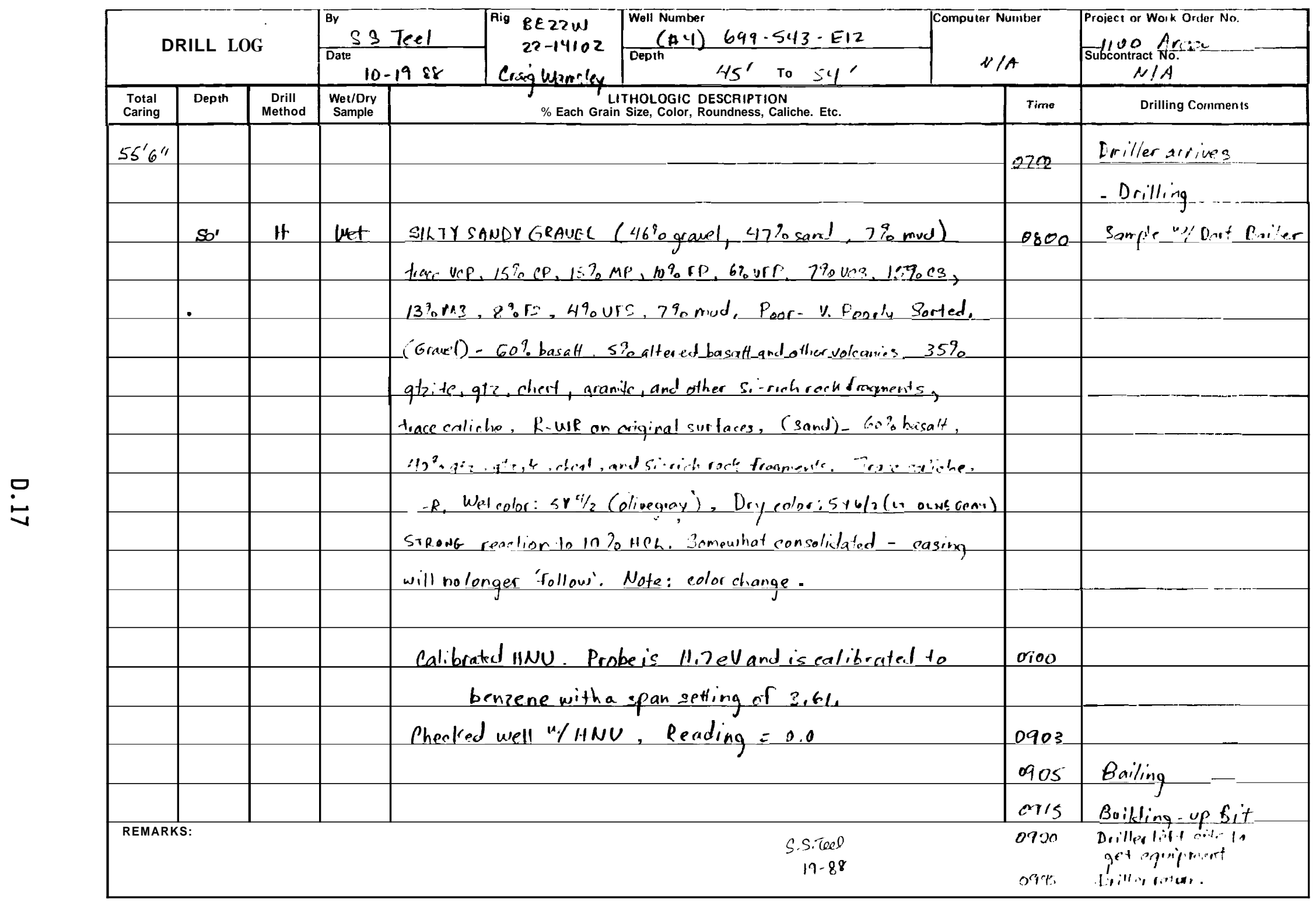

O- Drive Barrel H. Hard Tool L. Large M-Medium S-Small VC-Very Coarse C-Coarse F-Fine VF-Very Fine Standing Water 
$\therefore$ at 3

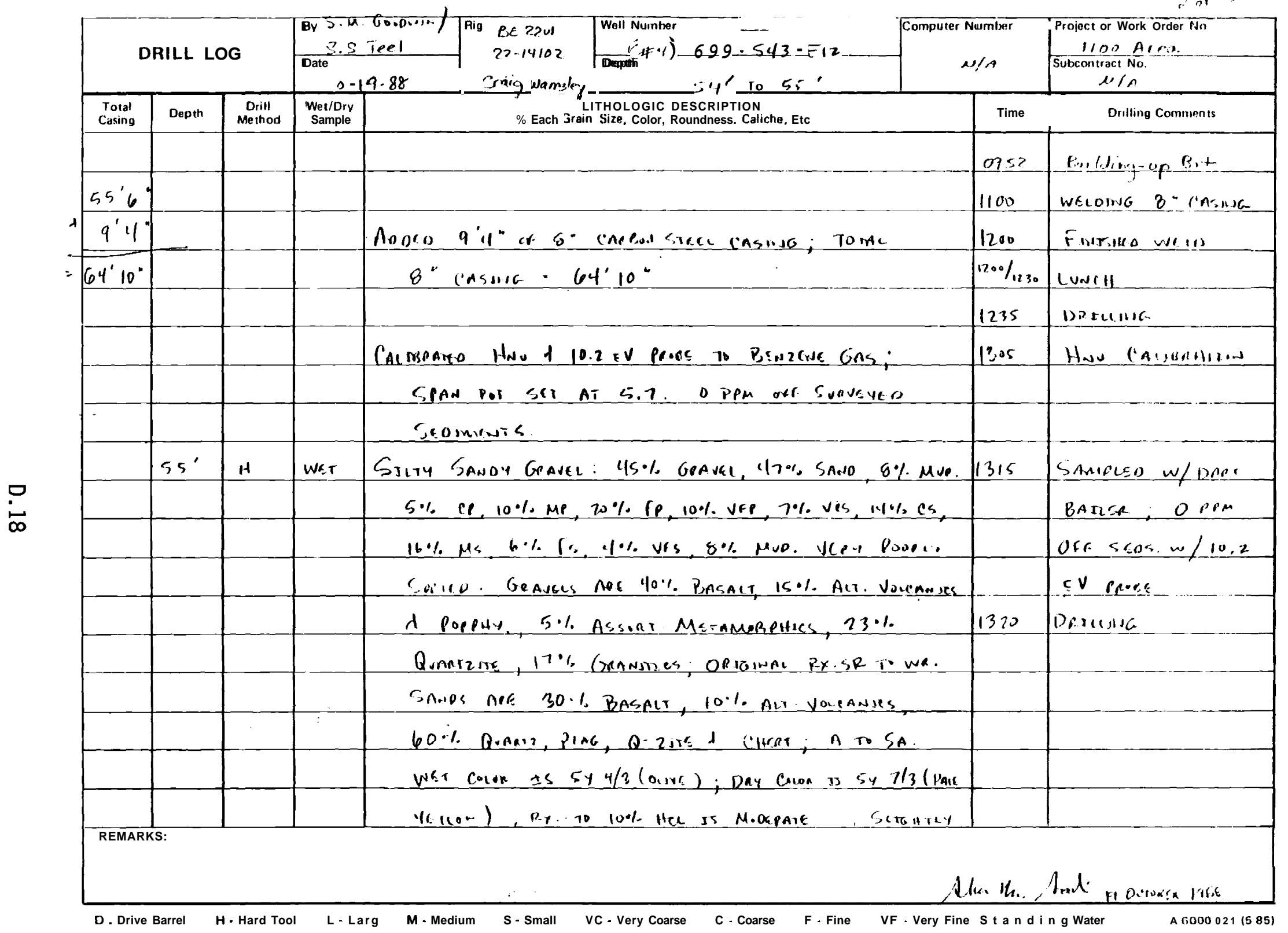


3 or 3

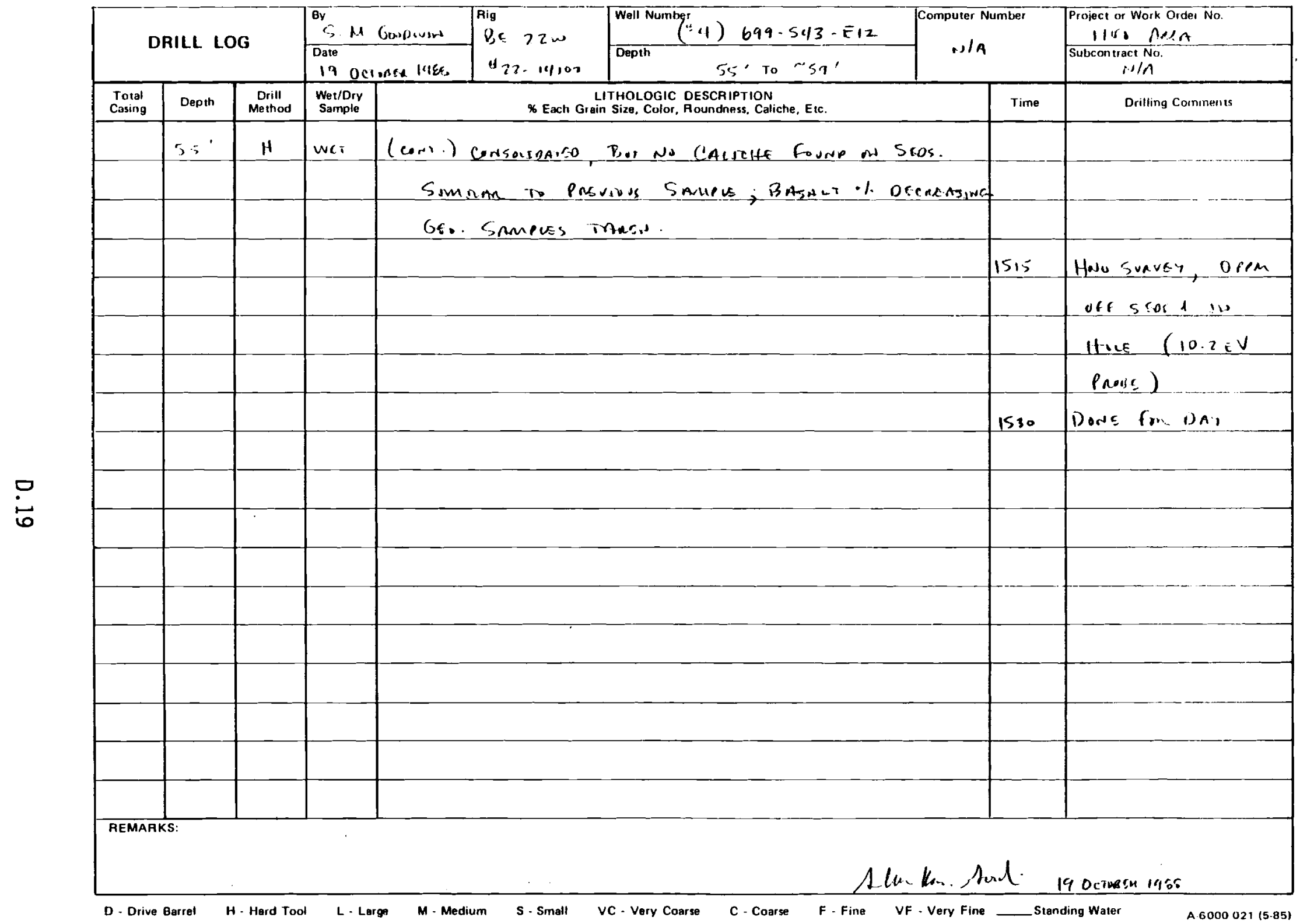


1 or 4

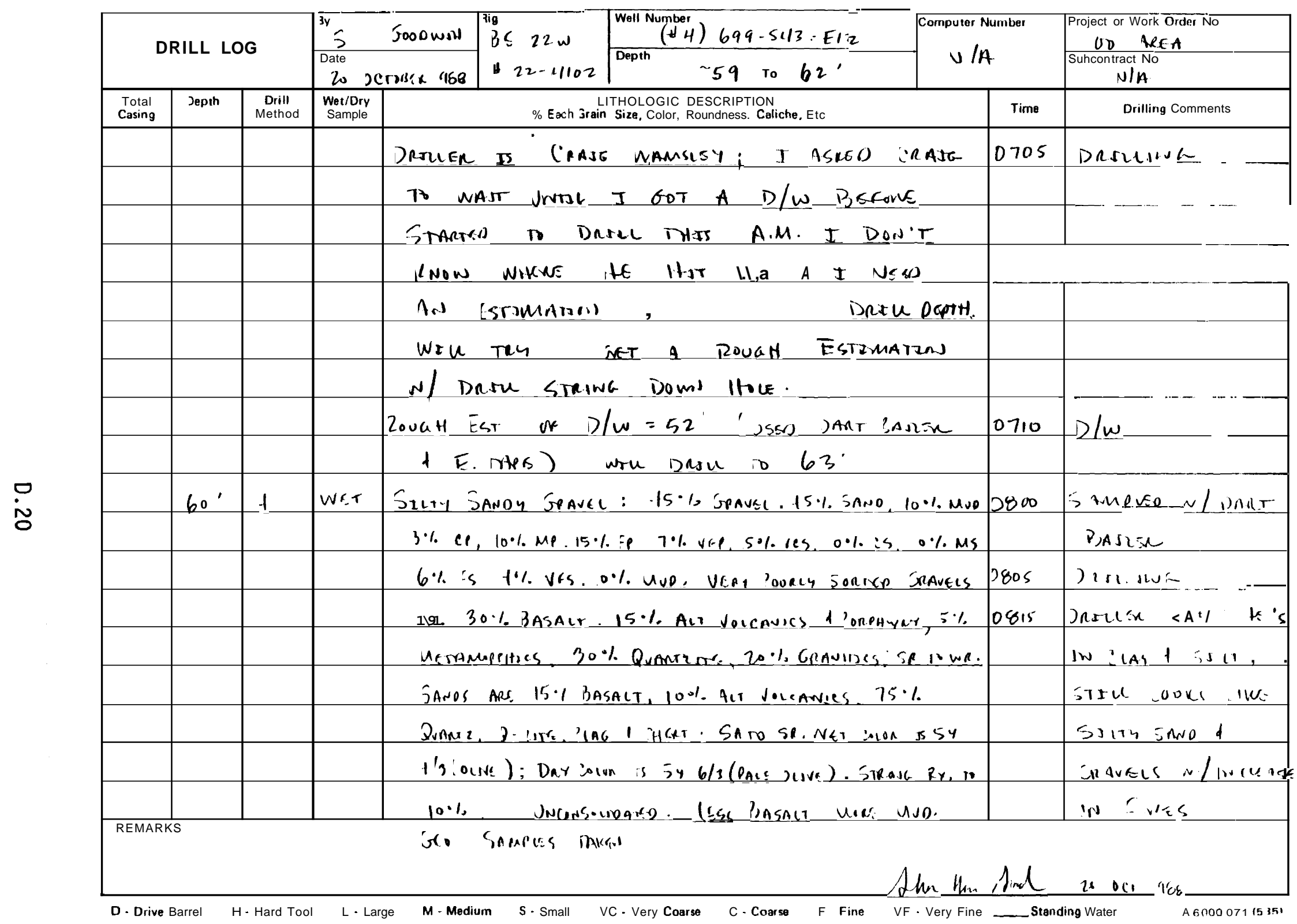




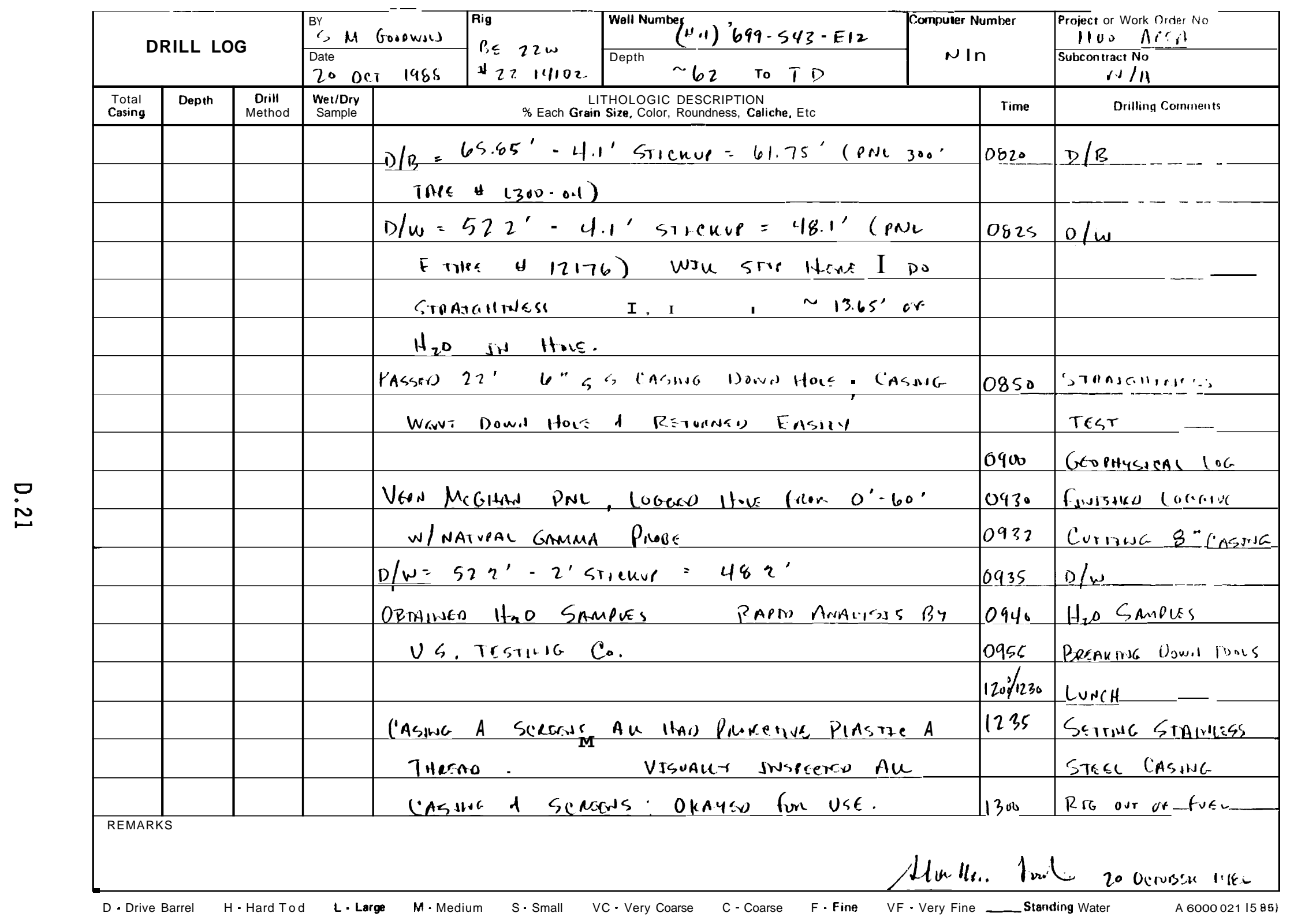


3 of 4

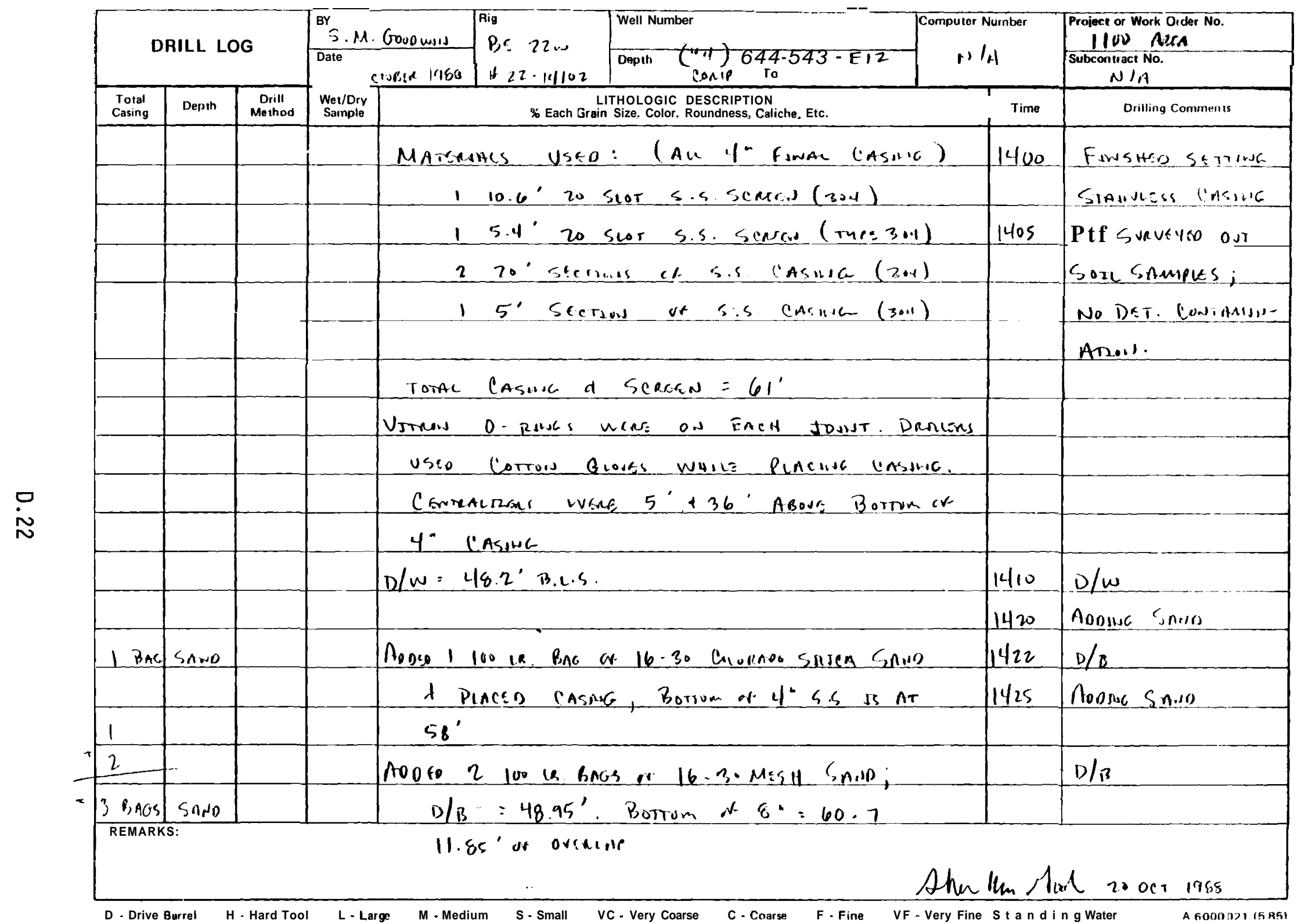


4 ur.

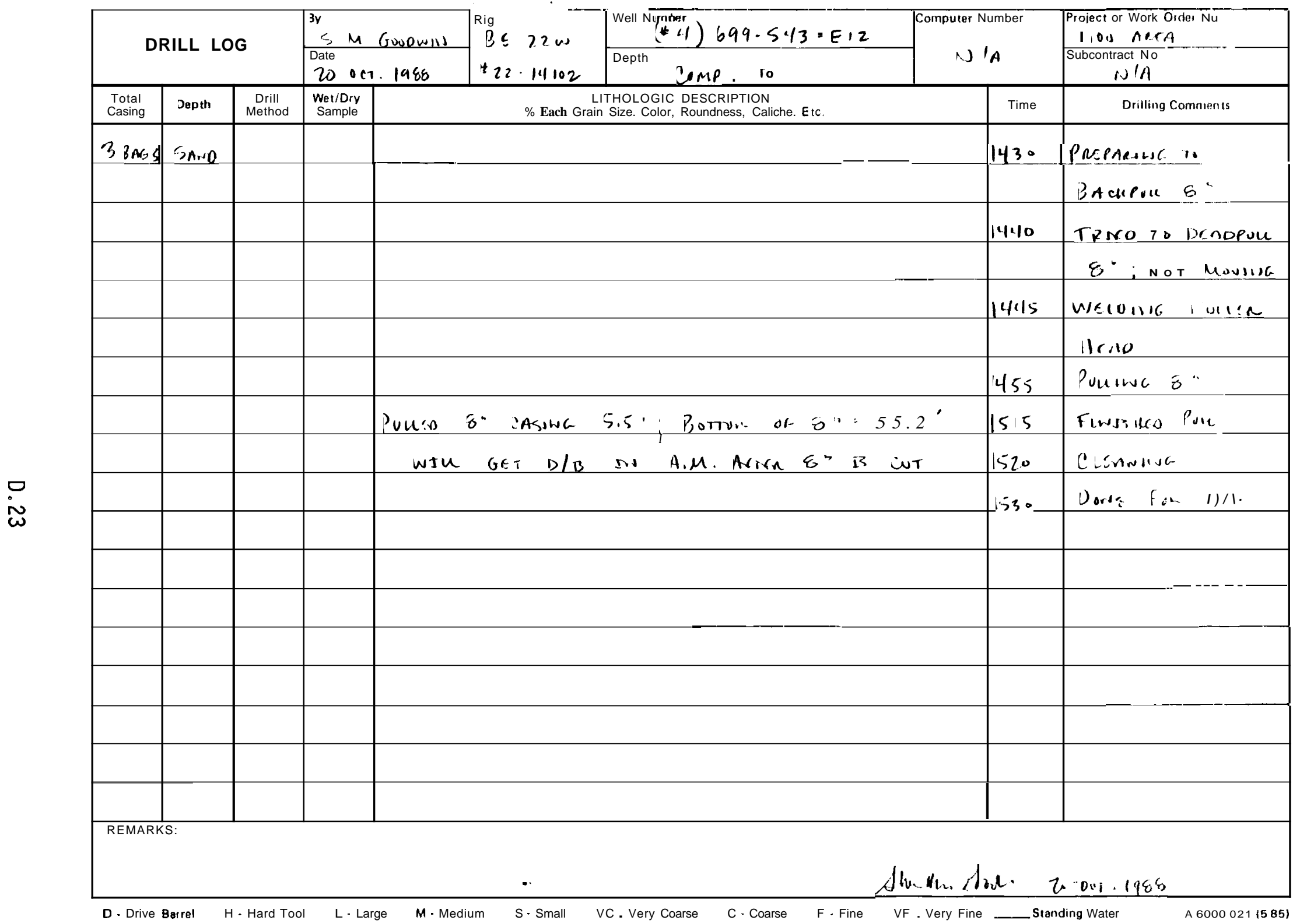


1011

\begin{tabular}{|c|c|c|c|c|c|c|}
\hline \multirow{2}{*}{\multicolumn{3}{|c|}{ DRILL LOG }} & \multirow{2}{*}{ 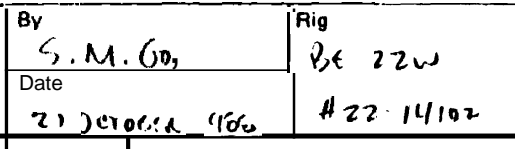 } & \multirow{2}{*}{ 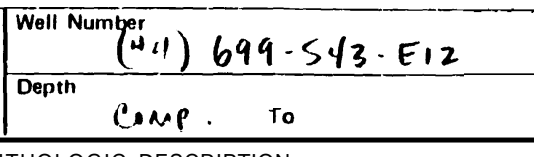 } & \multirow{2}{*}{$\begin{array}{c}\text { Conputer Number } \\
J / A\end{array}$} & \multirow{3}{*}{ 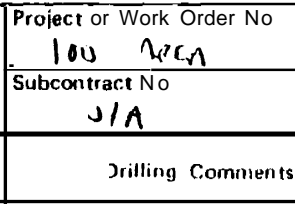 } \\
\hline & & & & & & \\
\hline \multirow[t]{4}{*}{$\begin{array}{l}\text { Total } \\
\text { Casing }\end{array}$} & Depth & $\begin{array}{l}\text { Drill } \\
\text { Method }\end{array}$ & $\begin{array}{l}\text { Wei/Dry } \\
\text { Sample }\end{array}$ & $\begin{array}{l}\text { LITHOLOGIC DESCRIPTION } \\
\% \text { Each 3rain Size, Color, Rounthess Caliche, Etc }\end{array}$ & Time & \\
\hline & & & & Drause is Conja wamsesy & 2700 & Eumerra ec" \\
\hline & & & & $2 / w=50.1-23 \cdot 5$ reke $=47.8^{\circ}$ l PNe $F$ & 2720 & $2 / w \ldots$ \\
\hline & & & & \multicolumn{2}{|l|}{$2270)$} & \\
\hline & & & & $D / B=53.05^{\prime} ;$ Bortom ov $8^{\circ} \cdot 55.2^{\circ}$ & 0125 & $D / B$ \\
\hline$\eta$ & & & & $215^{\prime} \mathrm{N}$ JukCLAP. Botruvh it $1^{n}=5725^{\circ}$ & 0740 & AODJwG GAND \\
\hline \multirow{2}{*}{\multicolumn{2}{|c|}{$\begin{array}{l}2 \text { 3atas } \\
5 \\
\end{array}$}} & & & 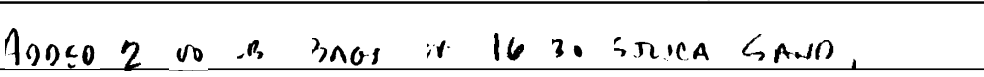 & 0745 & $2 / B$ \\
\hline & & & & $D / B=142^{\prime}, 11$ it overecne. & 675. & svectwa \&" \\
\hline & & & & $D\left(B^{\prime}\right.$ SAND $)=1775$, Botrom or $8^{\prime \prime}=502^{\prime}$ & 07 is & $D / B$ \\
\hline & & & & $2 a: ' x$ Jralue & 0757 & Aoouwe Snno \\
\hline 5 & & & & Adoso $n$ oD 18 BAGS of 16 3. MEST IAND. & 0800 & $D / B$ \\
\hline 2 & & & & $2 / B=39.05^{\circ}, 1.5^{\prime}$ in $\operatorname{lvencAp}$ & 0815 & 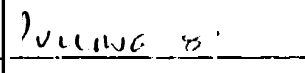 \\
\hline \multicolumn{2}{|c|}{73 A } & & & $D\left(1^{\prime}{ }^{\prime} S A_{N D}\right)=43.35^{\circ}$. BorTom N 8 15.2' & 0815 & $12 / B$ \\
\hline & & & & \multicolumn{2}{|c|}{\begin{tabular}{l|l}
$15^{\prime}$ of Ifrecte. & osin \\
\end{tabular}} & Doswg inell) \\
\hline-7 & & & & 2100 B ings r. 162015511 innes) & $78_{2} 0^{\circ}$ & $0 / 13$ \\
\hline$?$ & & & & 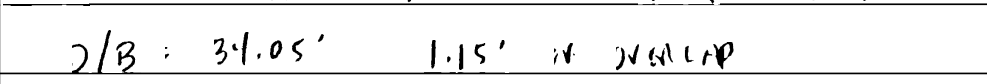 & $\underline{0825}$ & Parance $\&$ \\
\hline A & & & & $2 / B^{\prime}$ SAND $)=39200^{\prime} ;$ B.-TOM O० $0^{\prime \prime} 39 \geq 0$ & 784 & $0 / 0$ \\
\hline & & & & $D / B\left(4^{\prime \prime}\right) \cdot 5665^{\prime} \cdot 1.05^{\prime} \rightarrow 4 \quad 1^{\prime \prime}$ smincess & 0350 & Vf. $(x)=1,10$ \\
\hline REMARKS & & & & Ani Bign lovirle a v' julls & 10900 & Inowe ine \\
\hline \multicolumn{7}{|c|}{$\theta l x \cdot l_{x}}$. \\
\hline
\end{tabular}


.14

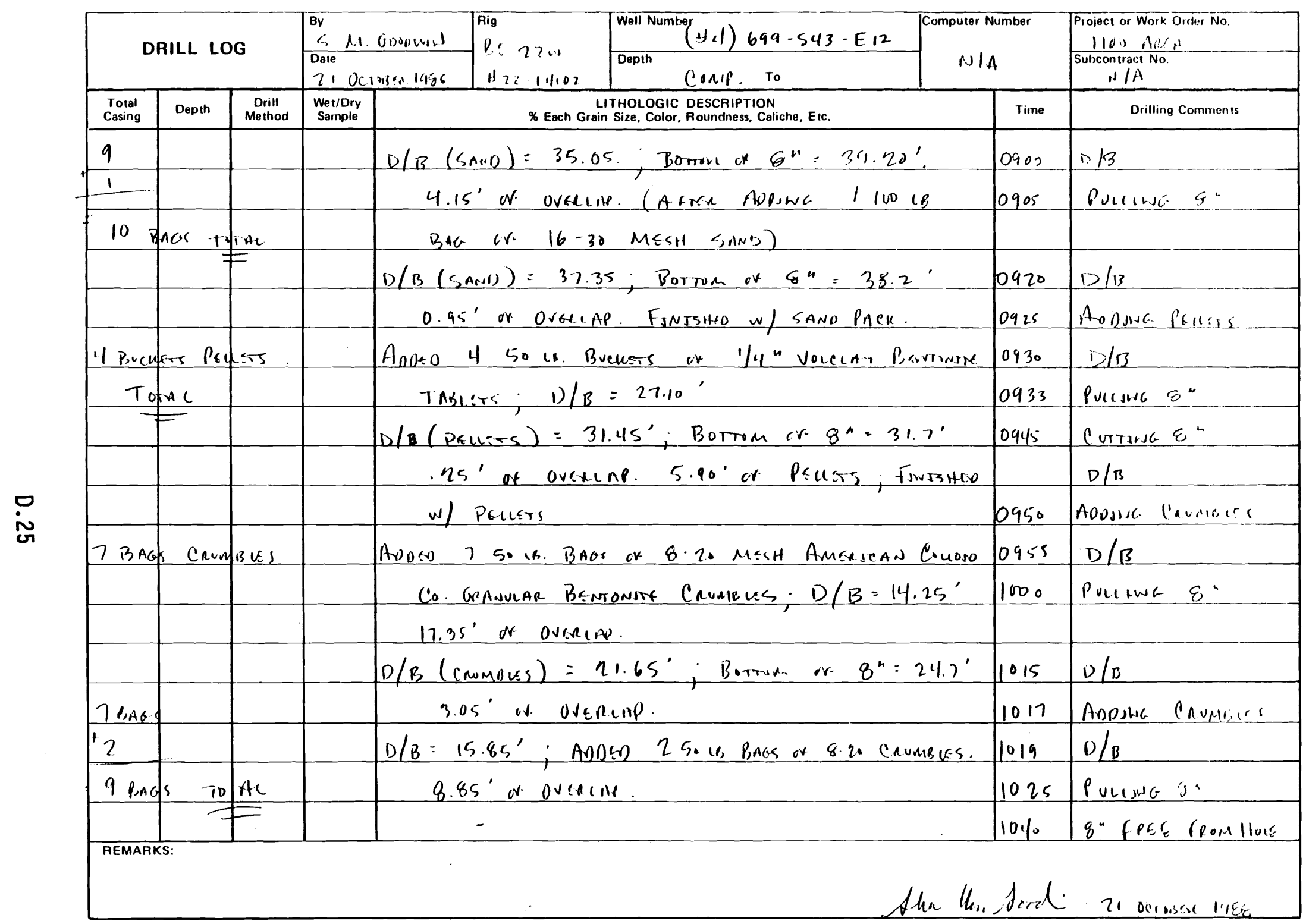

D. Drive Barrel H- Hard Tool L-Large M-Medium S-Small VC-Very Coarse C-Coarse F- Fine VF-Very Fine _ Standing Water A.600u 021 (5-85) 


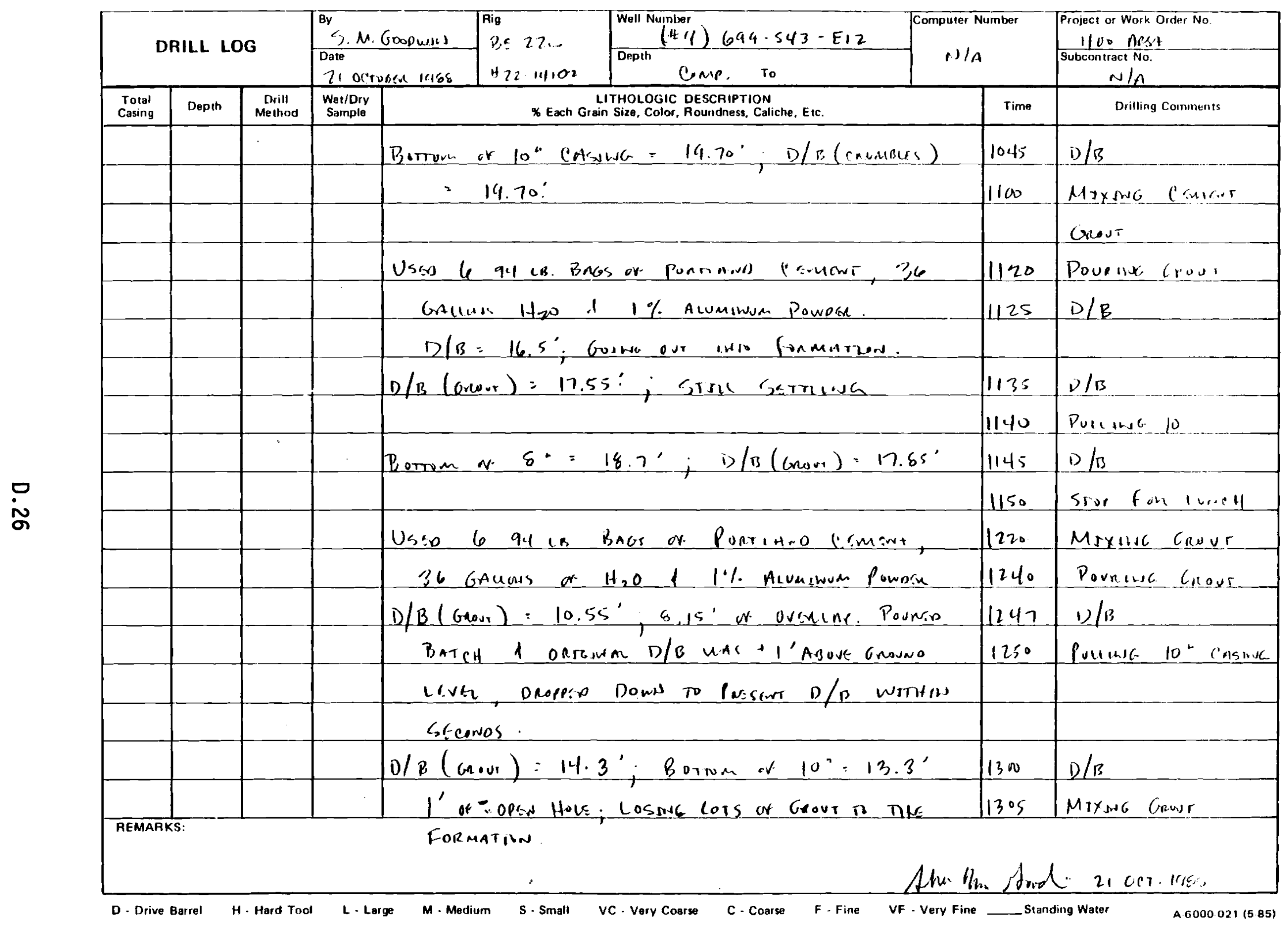




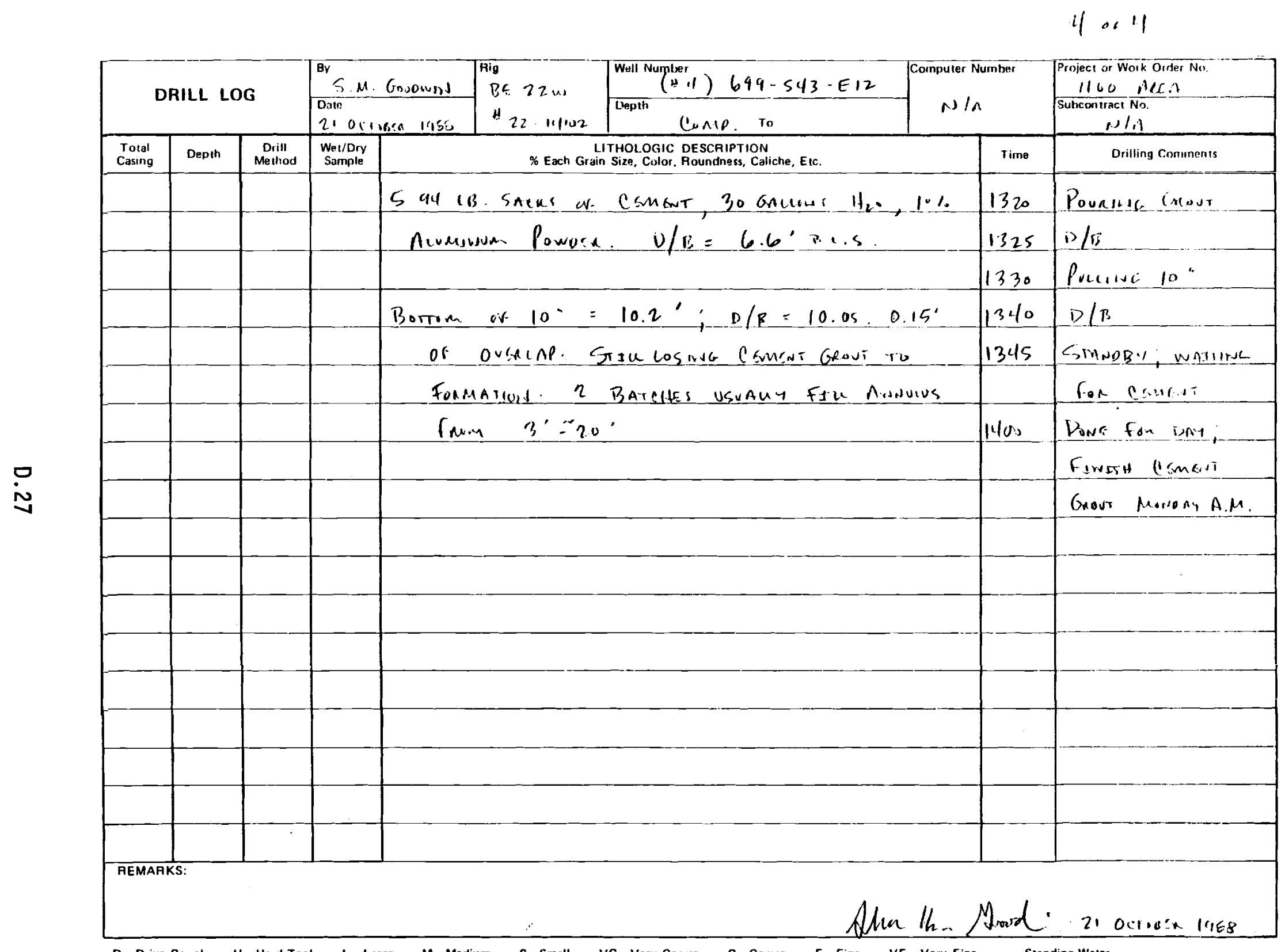

D.Drive Barrel H. Hard Tool L-Large M-Medium S-Small VC-Very Coarse C-Coarse F.Fine VF-Very Fine _- Standing Water 


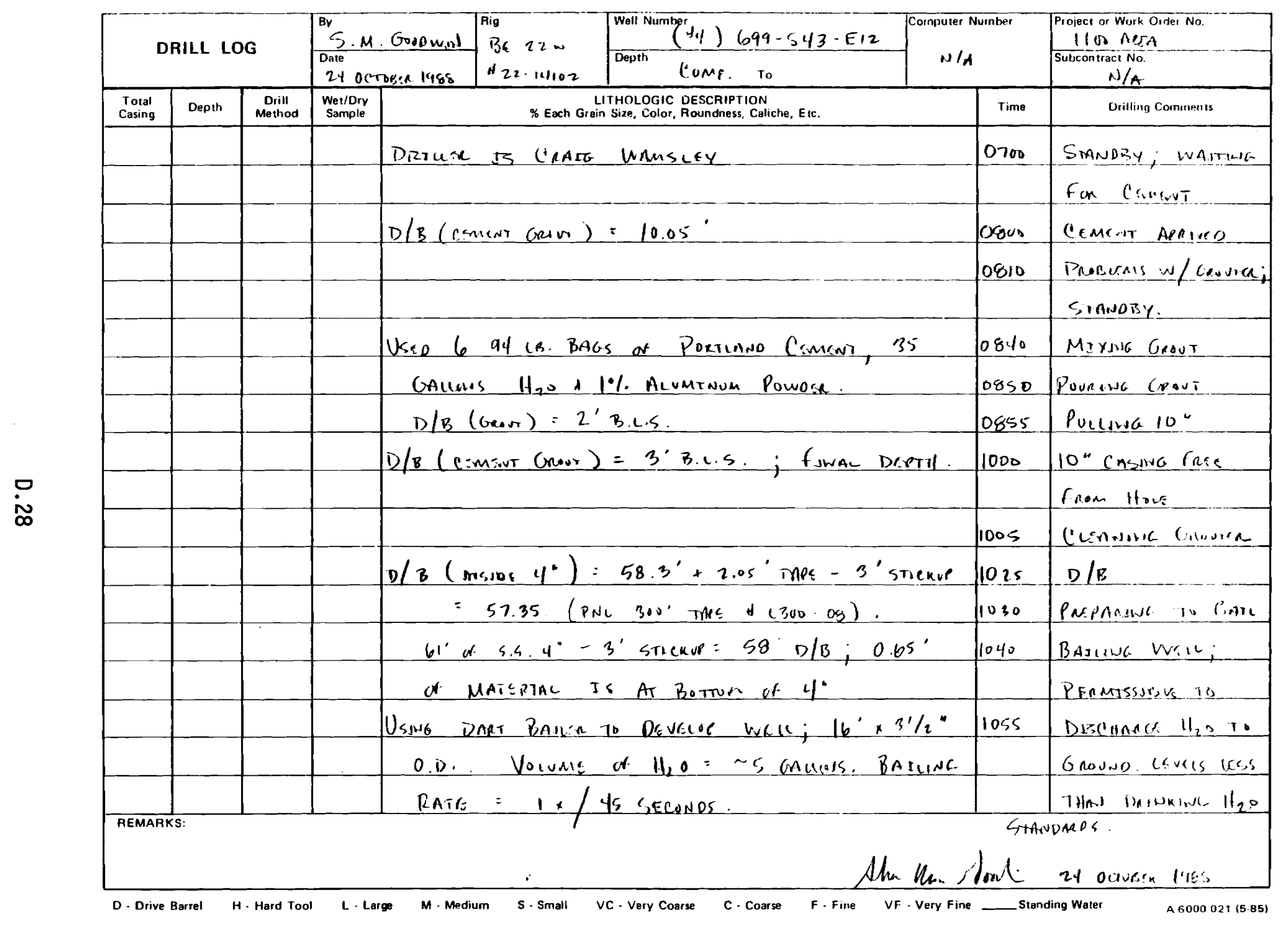


7 ra

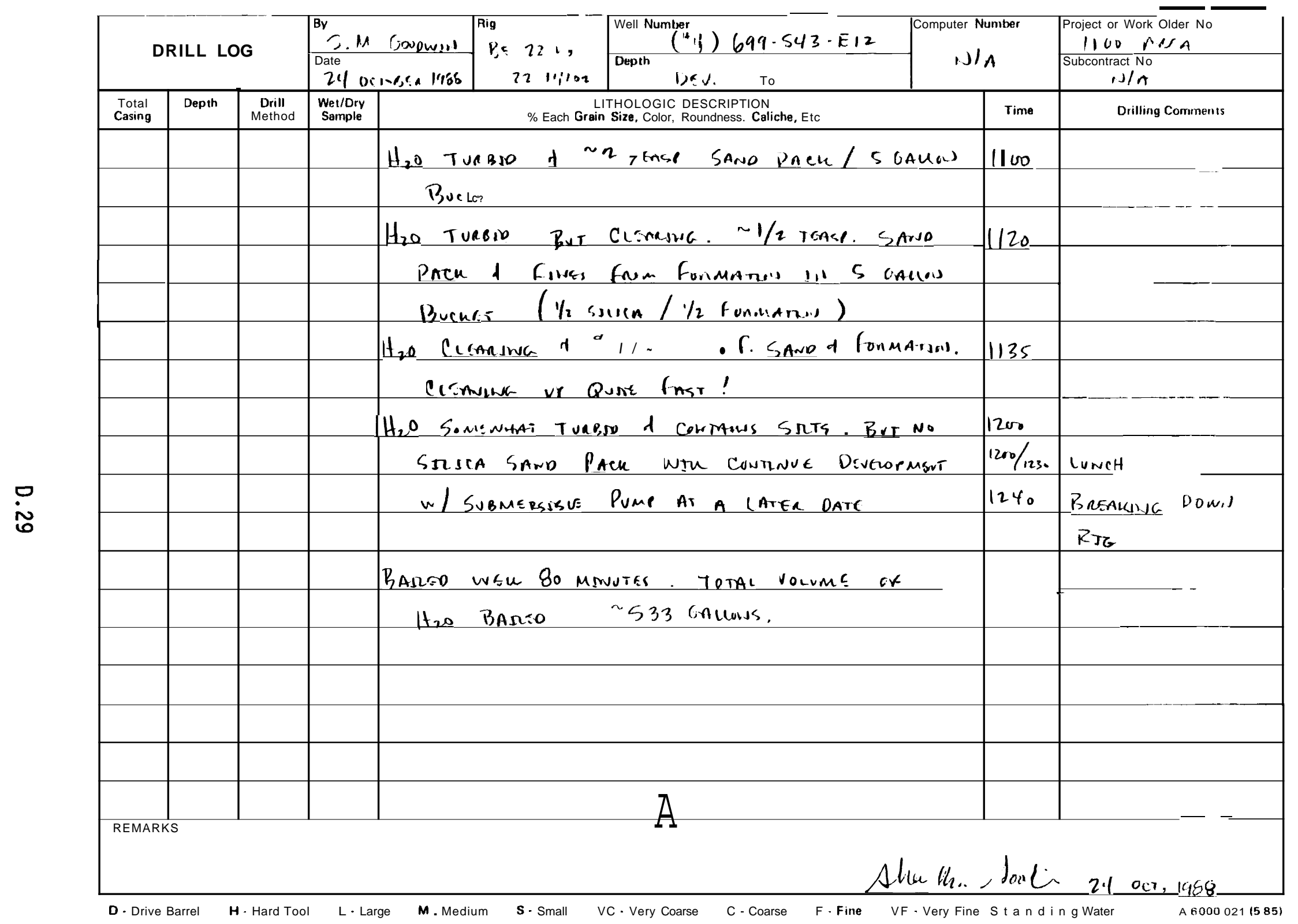


$1 n+1$

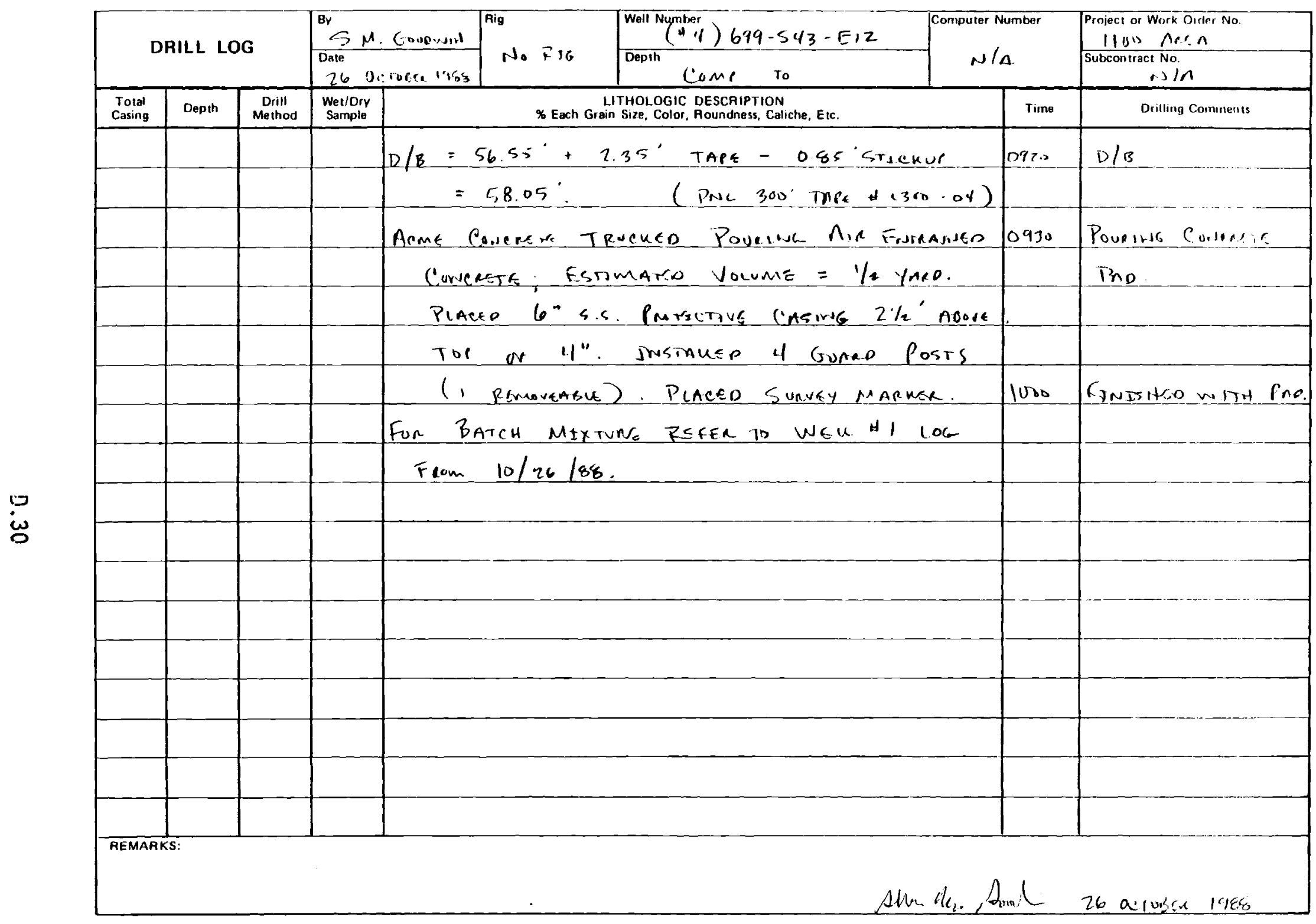

D- Drive Barrel H. Hard Tool L-Large M-Medium S-Small VC-Very Coarse C.Coarse F-Fine VF-Very Fine _- Standing Water A 6000 021 (585) 
101

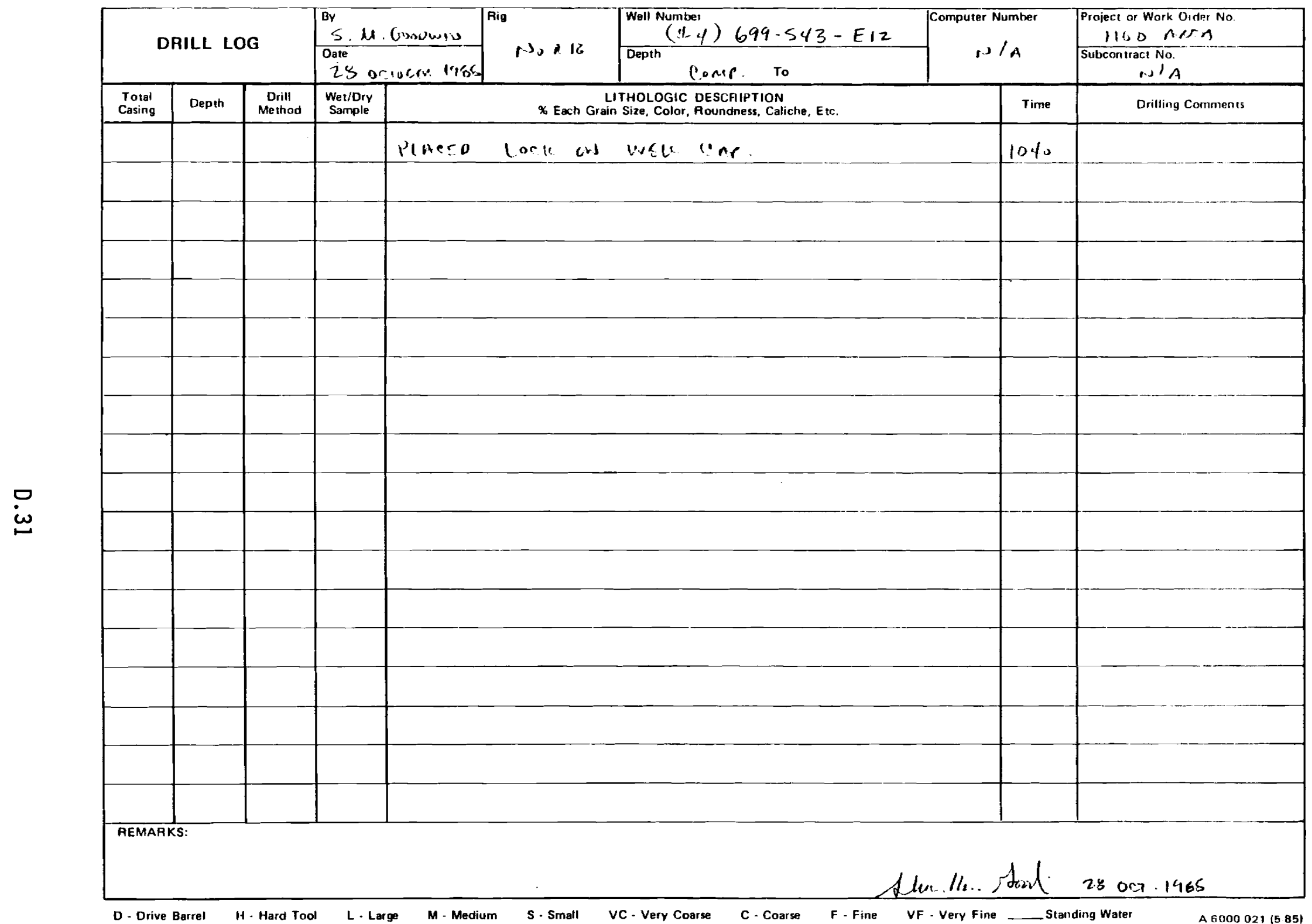




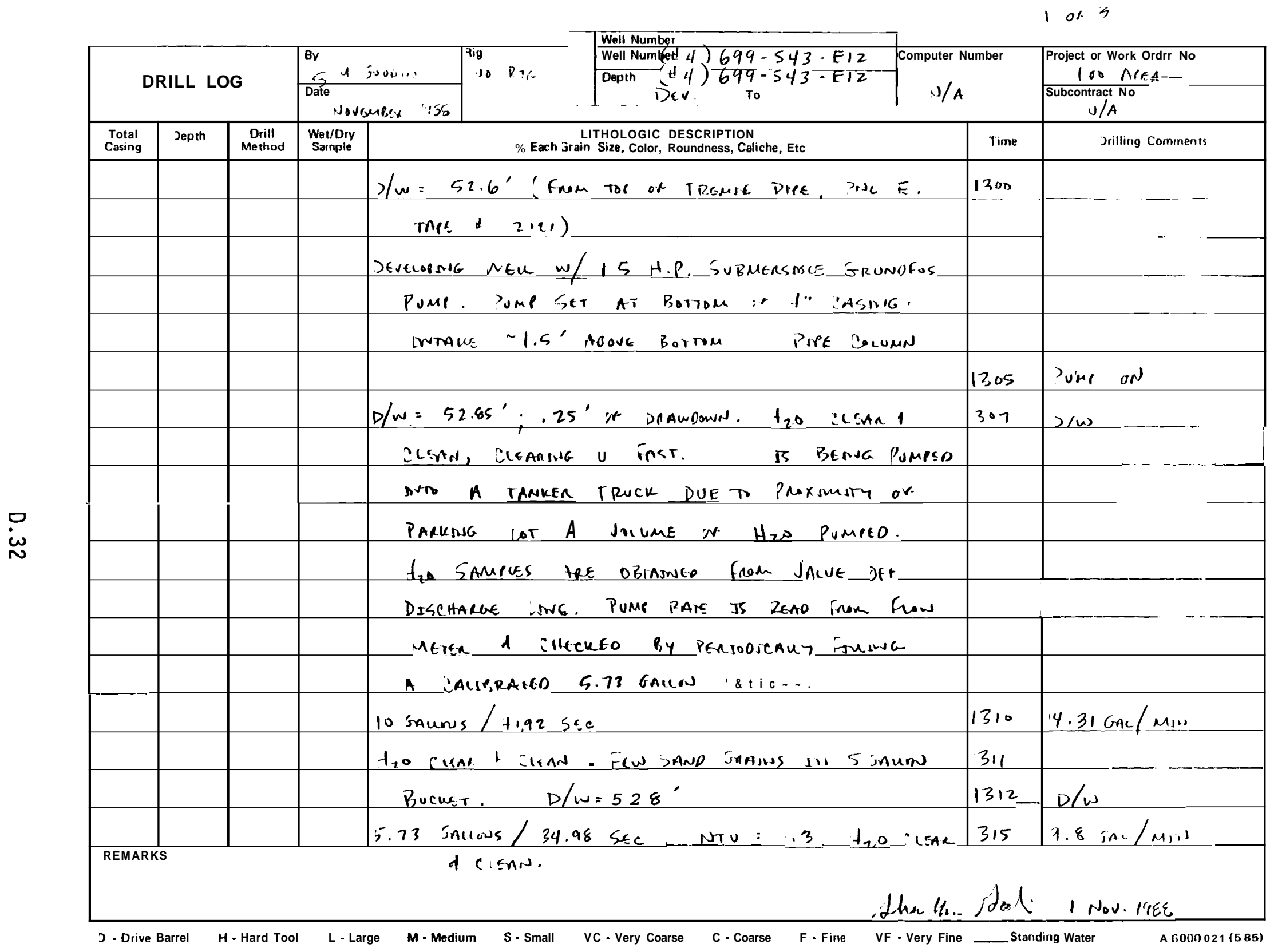


2 of ?

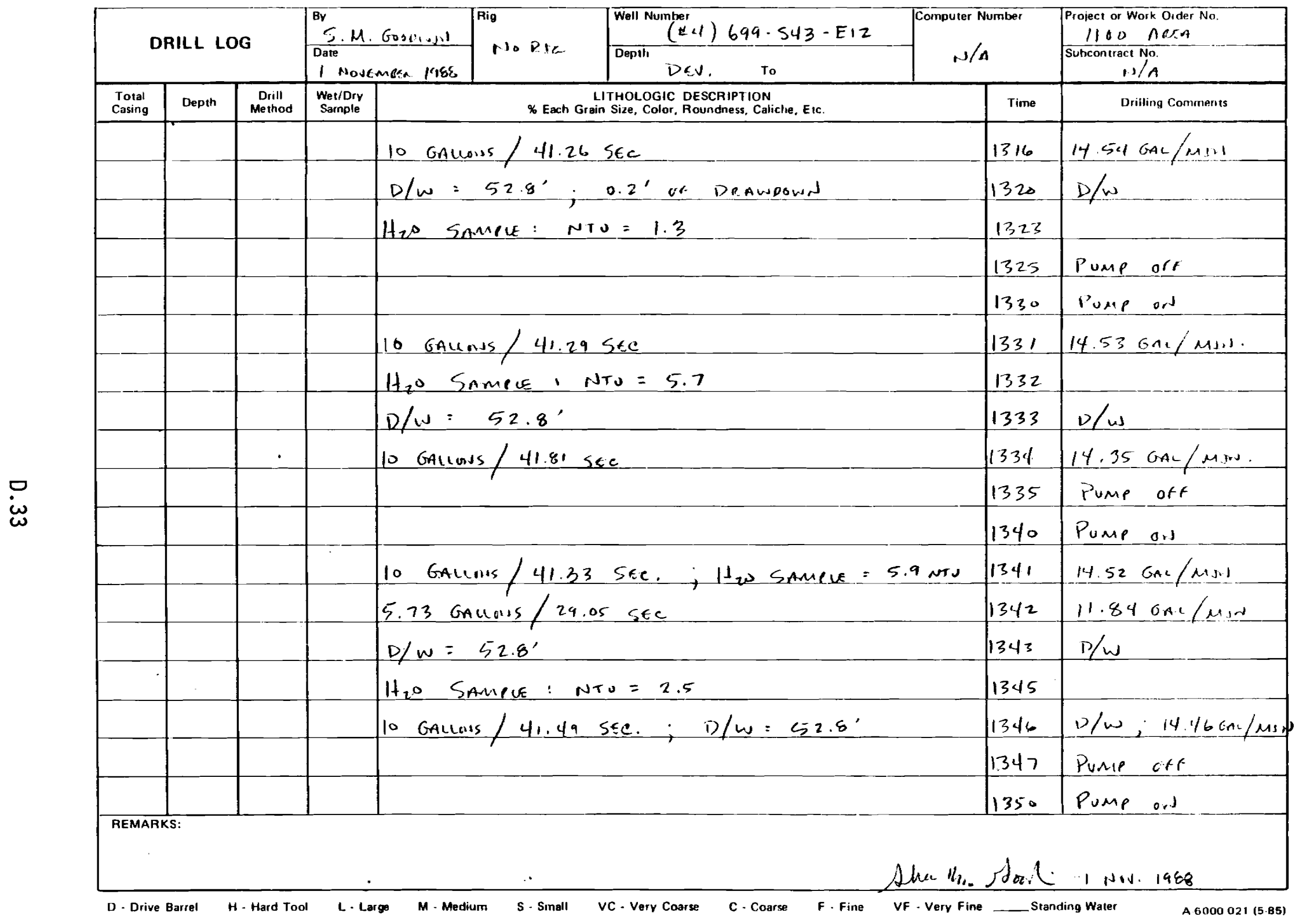




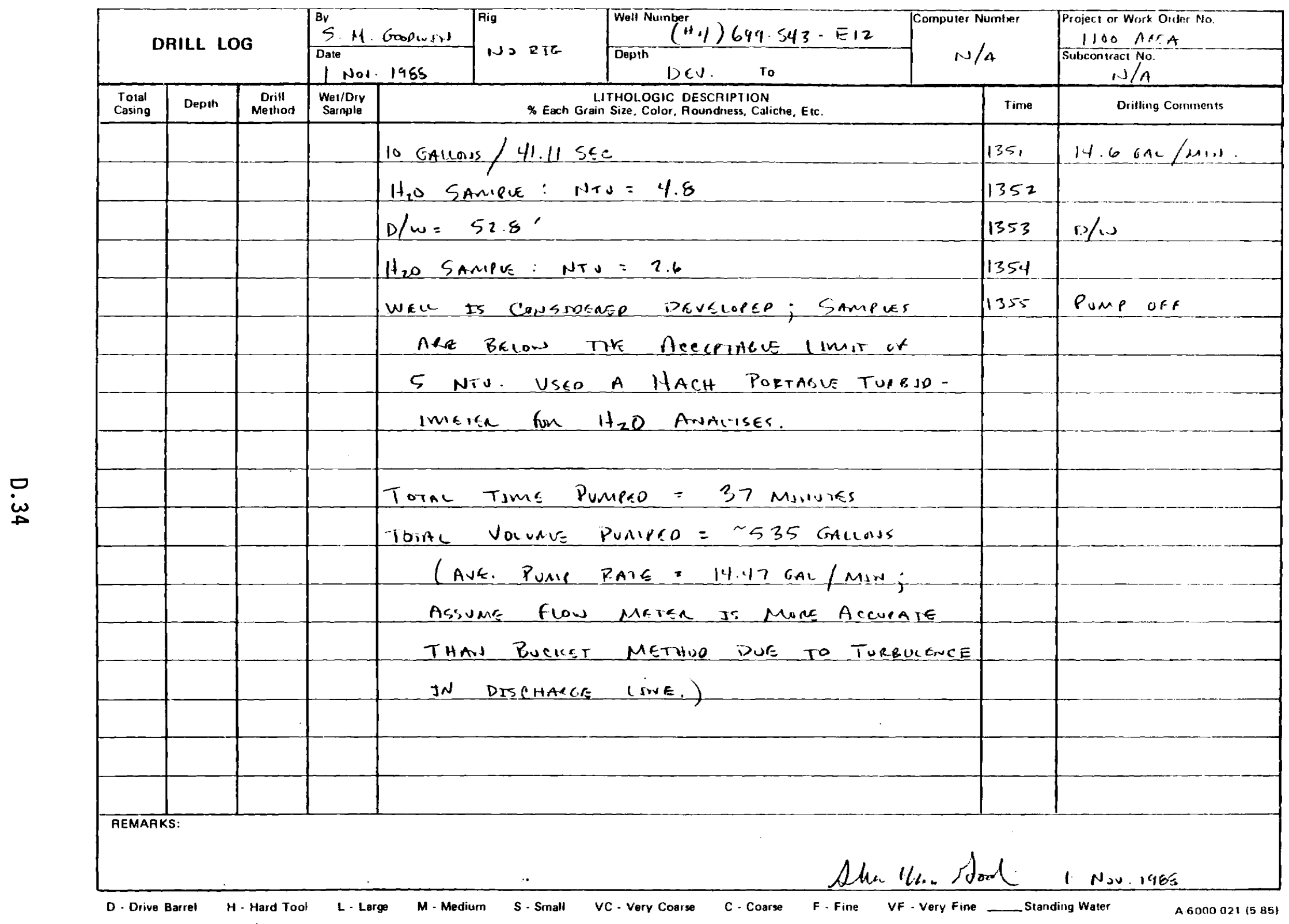




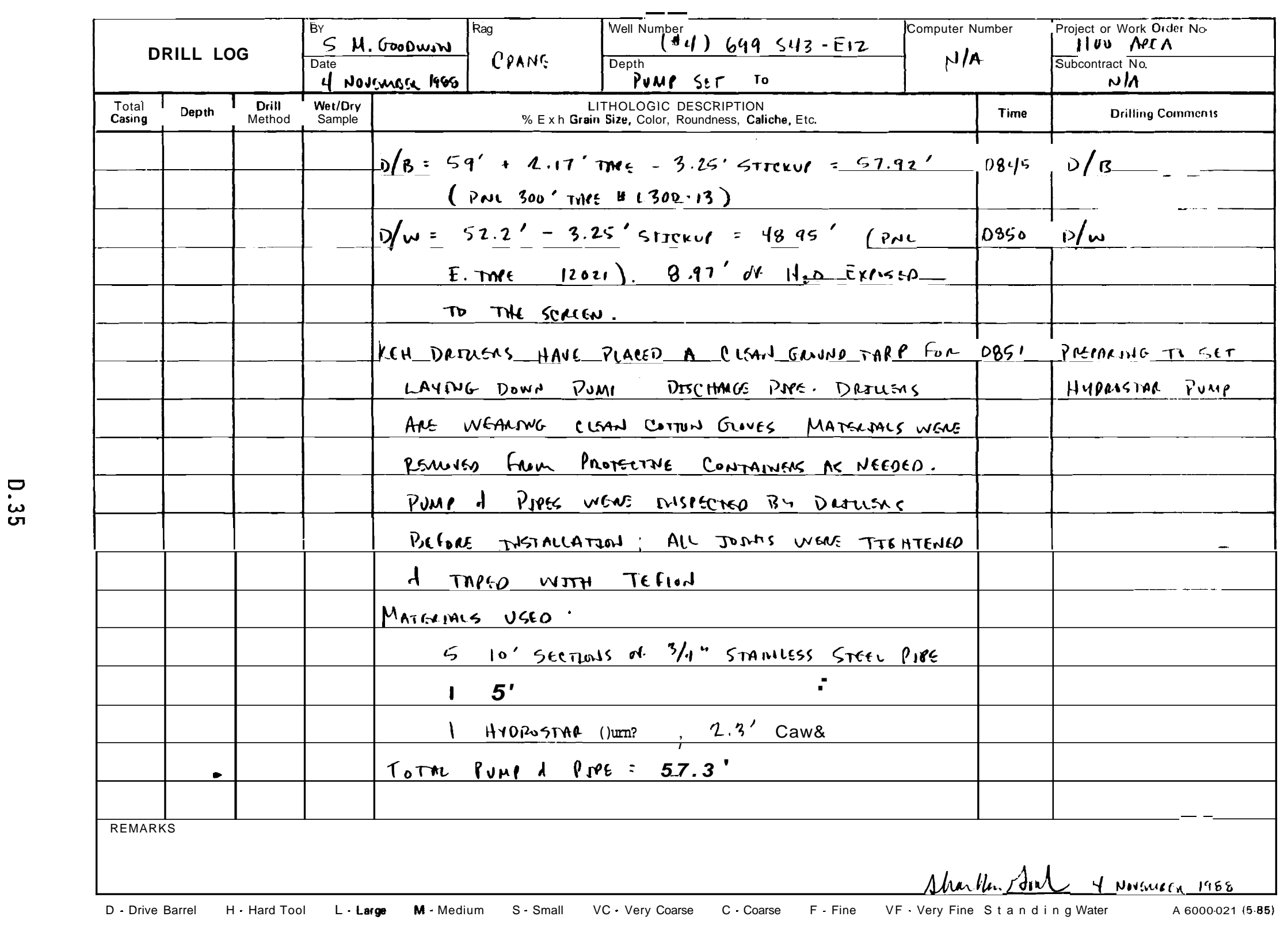




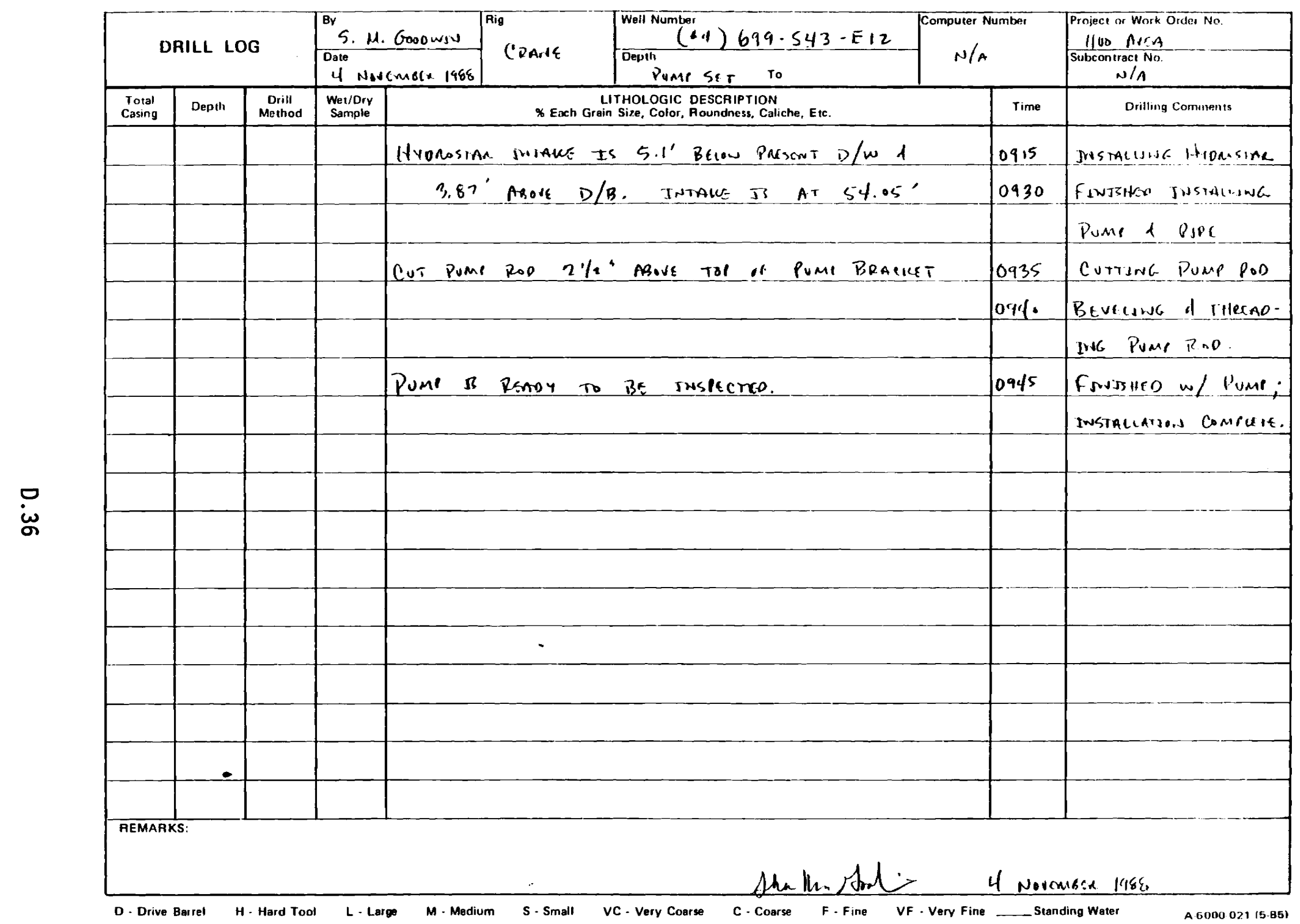




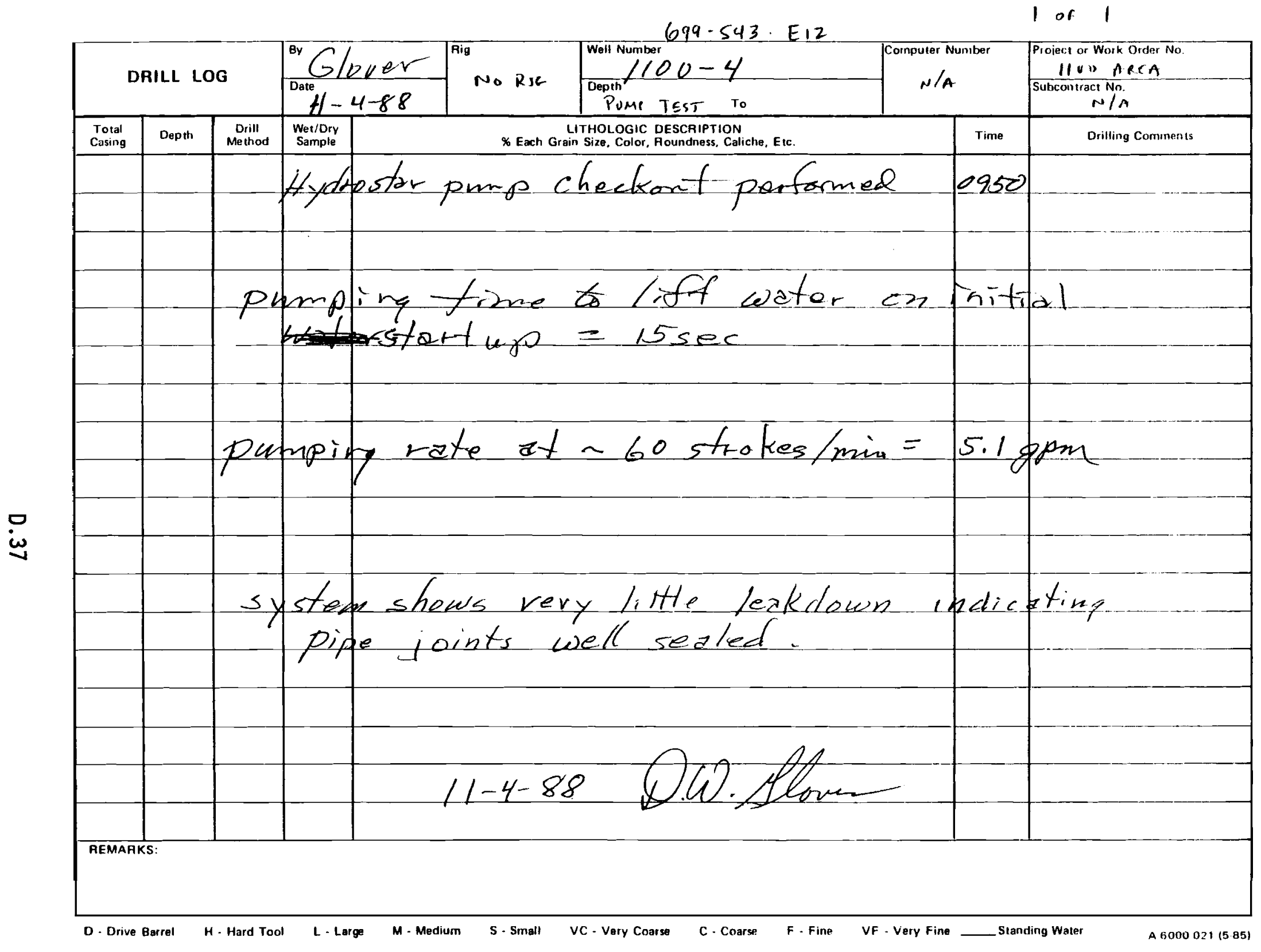




\section{APPENDIX E}

GEOPHYSICAL AND GEOLOGIC LOGS FOR WELL 5 (699-S37-E14) 


\section{APPENDIX E}

\section{GEOPHYSICAL AND GEOLOGIC LOGS FOR WEL 5 (699-S37-E14)}

This appendix contains the Well Completion/Inspection Report, as-built diagram, notes from the sampling pump installation, the natural gamma log, and the geologists' logs for well 5 (699-S37-E14) in the 1100 Area. 


\begin{tabular}{|c|c|}
\hline \multicolumn{2}{|c|}{ HELL COMPLETION/INSPECTION REPORT } \\
\hline 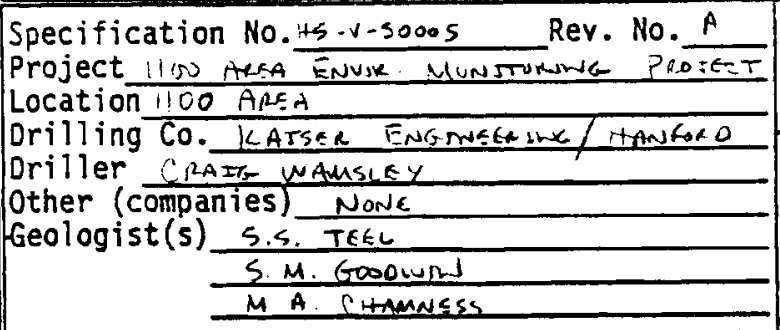 & 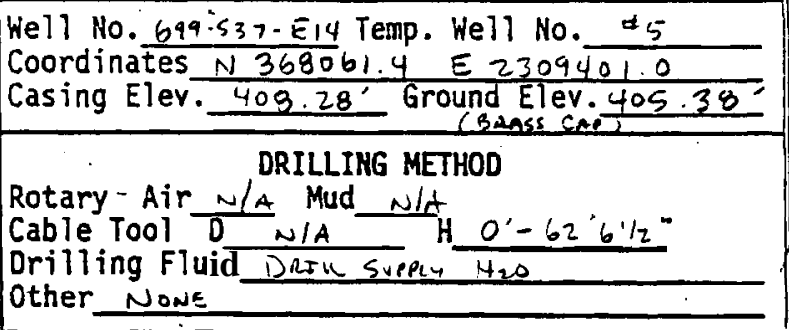 \\
\hline $\begin{array}{c}\text { GEOPHYSICAL LOGGING } \\
\text { Sondes } \\
\text { Ninterval Date } \\
=\square\end{array}$ & 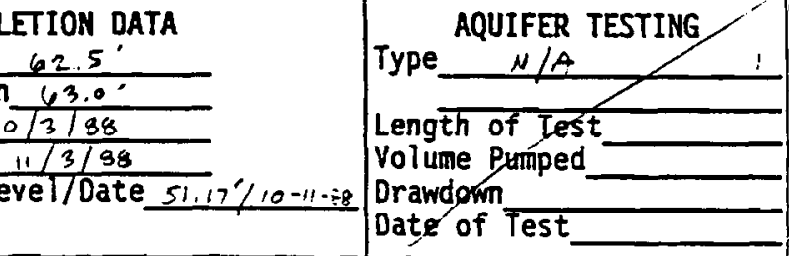 \\
\hline 1 & \\
\hline 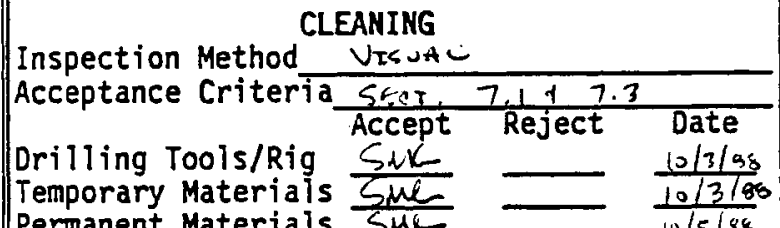 & $\begin{array}{l}\text { MATERIAL STORAGE/PACKING } \\
\text { Inspection Method } \bigcup_{\text {ISUAC }} \\
\text { Acceptance Criteria SECT. } 7.3 \\
\text { Mt 1. Handling/Storage SMUPt Reject Date } \\
\text { Material Packing }\end{array}$ \\
\hline 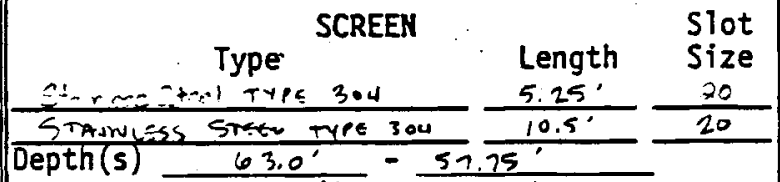 & $\begin{array}{l}\text { Inspection Method VJSun } \\
\text { Acceptance Criteria SEcT. } 7.2 \\
\text {. Identify Accept Reject Date } \\
\text { Additives Nove : } \\
\text { Lubricants Food ost }\end{array}$ \\
\hline $\begin{array}{l}\text { Inspection Method Visual } \\
\text { Acceptance Criteria SEcT.4.2.3 } \\
\text { Accept } \leq=T \text { Reject Date } 10 / 11 / 84\end{array}$ & 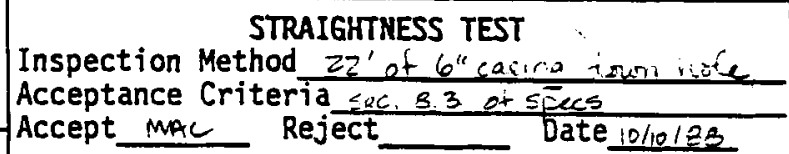 \\
\hline 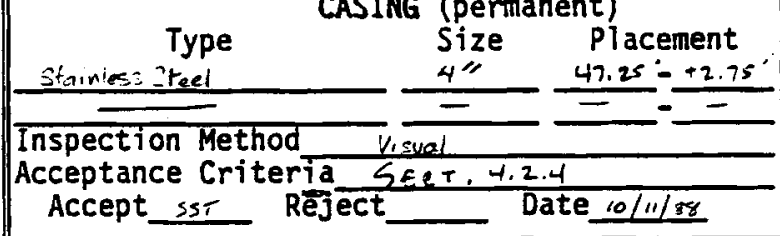 & 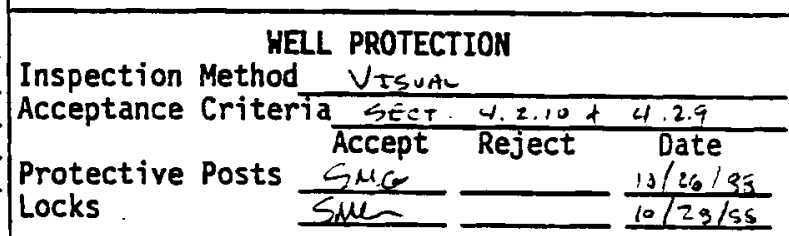 \\
\hline 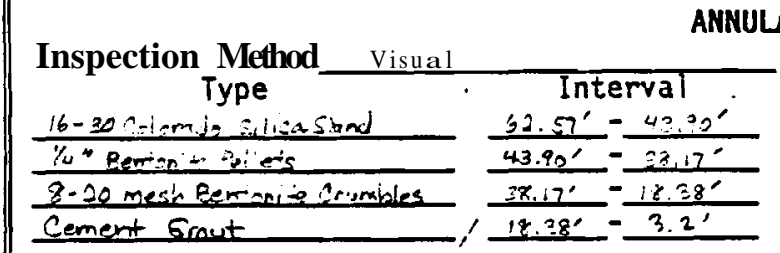 & 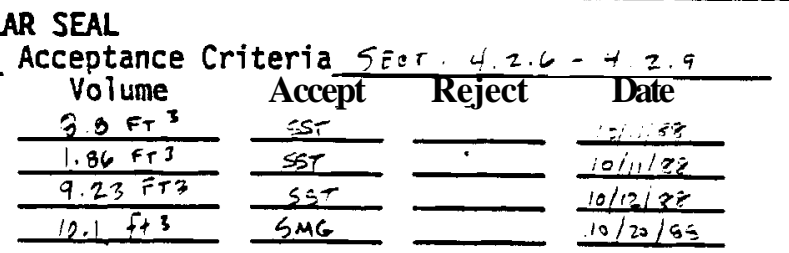 \\
\hline $\begin{array}{l}N / A \\
\text { SML Well Abandonment } \\
\text { Hell Development }\end{array} \quad \mathrm{N} / \mathrm{A}$ Downhole TV In & $\begin{array}{l}\text { IER (initial if performed) } \\
\text { Ispection } \leq \text { Complete As-Built Diagram, } \\
\text { Driller's/Geologist's Logs }\end{array}$ \\
\hline
\end{tabular}

REVIEwES By V.Thrablem 11-8-88

For all blanks mak N/A if not applicable. 


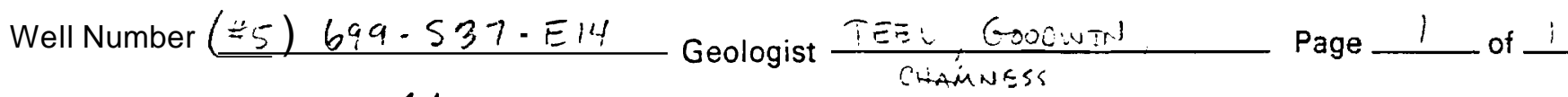
Reviewed by v.hWe Lhan Date $11-8-88$

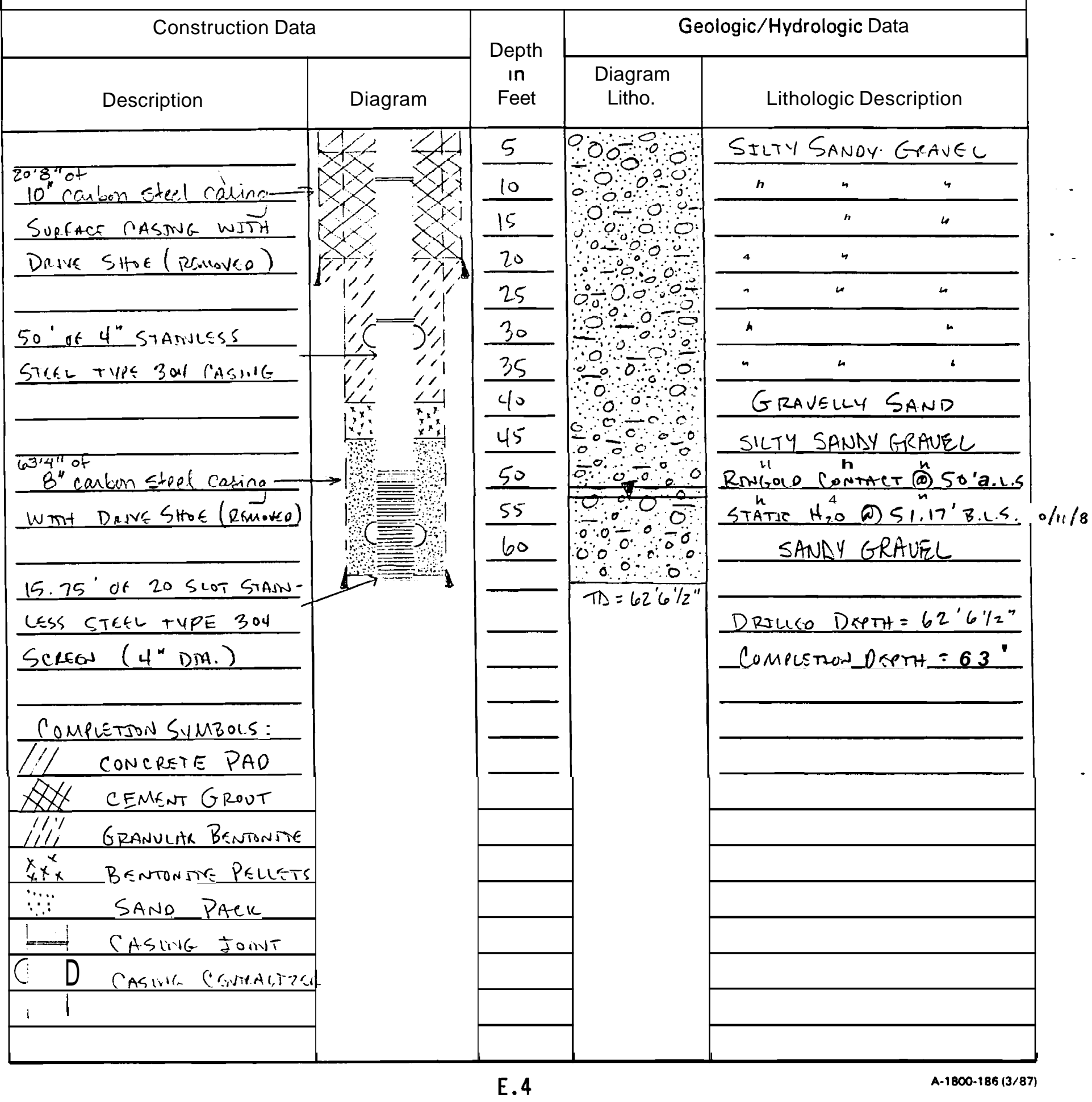


SAMPLING PUMP INSTALIATION IN

GROUND-WATER MONITORING WELLS

Site: $\quad 1100$ AREA

Monitoring Hell Number: $(\# 5) 699-537^{-}$E14

Depth to Water: $53.3^{\prime}$

Depth to Bottom: $63.10^{\circ}$ Reported Depth to Bottom: $63.0^{\circ}$

Pump Type: Positive Displacement Piston

Pump Model: Hydrostar HS-8001

Date Installed: $11 / 3 / 85$

Installed By: KEH: OLN Amos Lovs WATKSWS, LENNY COROON

Pump Discharge Pipe Description:

$610^{\circ}$ SECTIDNS of $3 / 4^{\prime \prime}$ STAMLSS STEEL DESCHARE PJPE.

Additional Comments:

HYDROSTAR PUMP LENGTH $=2.3^{\prime}$; ALL JOAHTS WERE TAPED

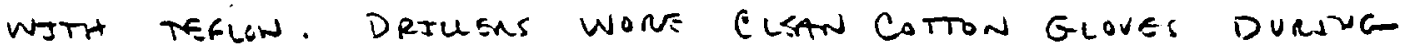

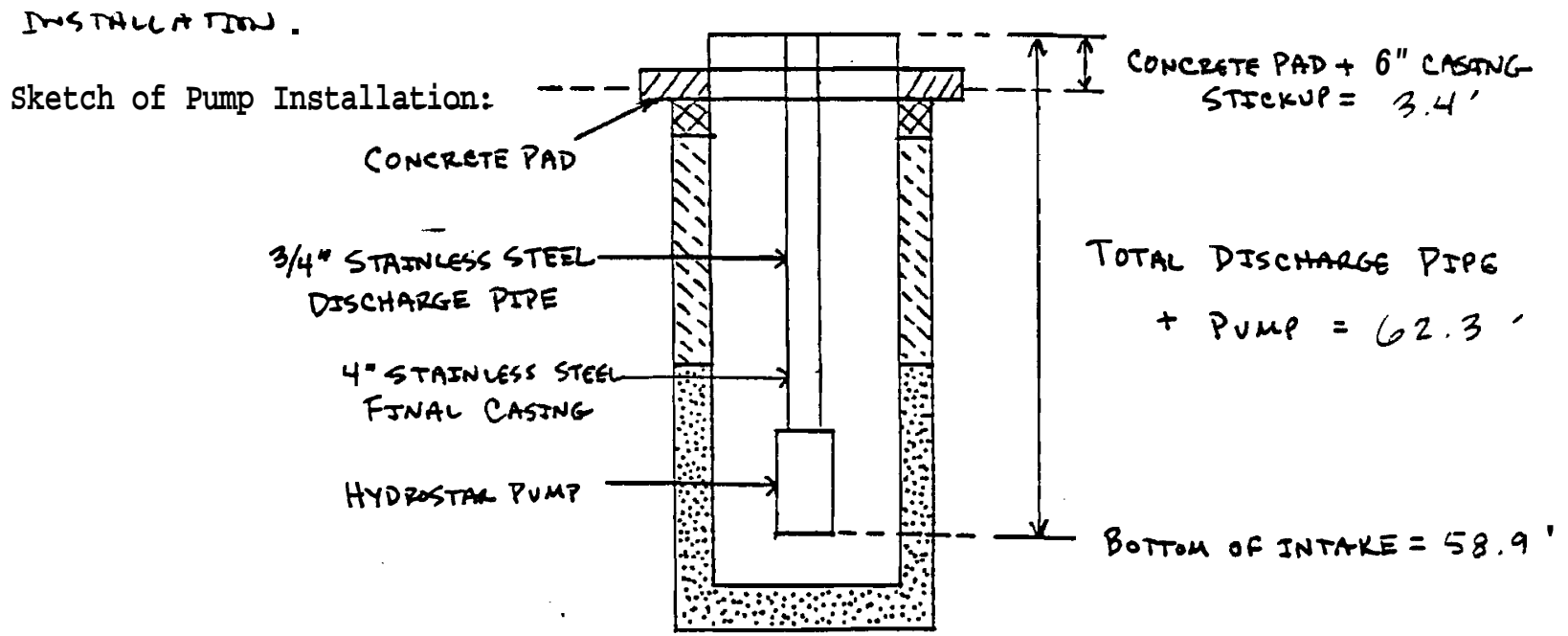

Completed By: Shanthr Note: $11 / 3 / 88$ 


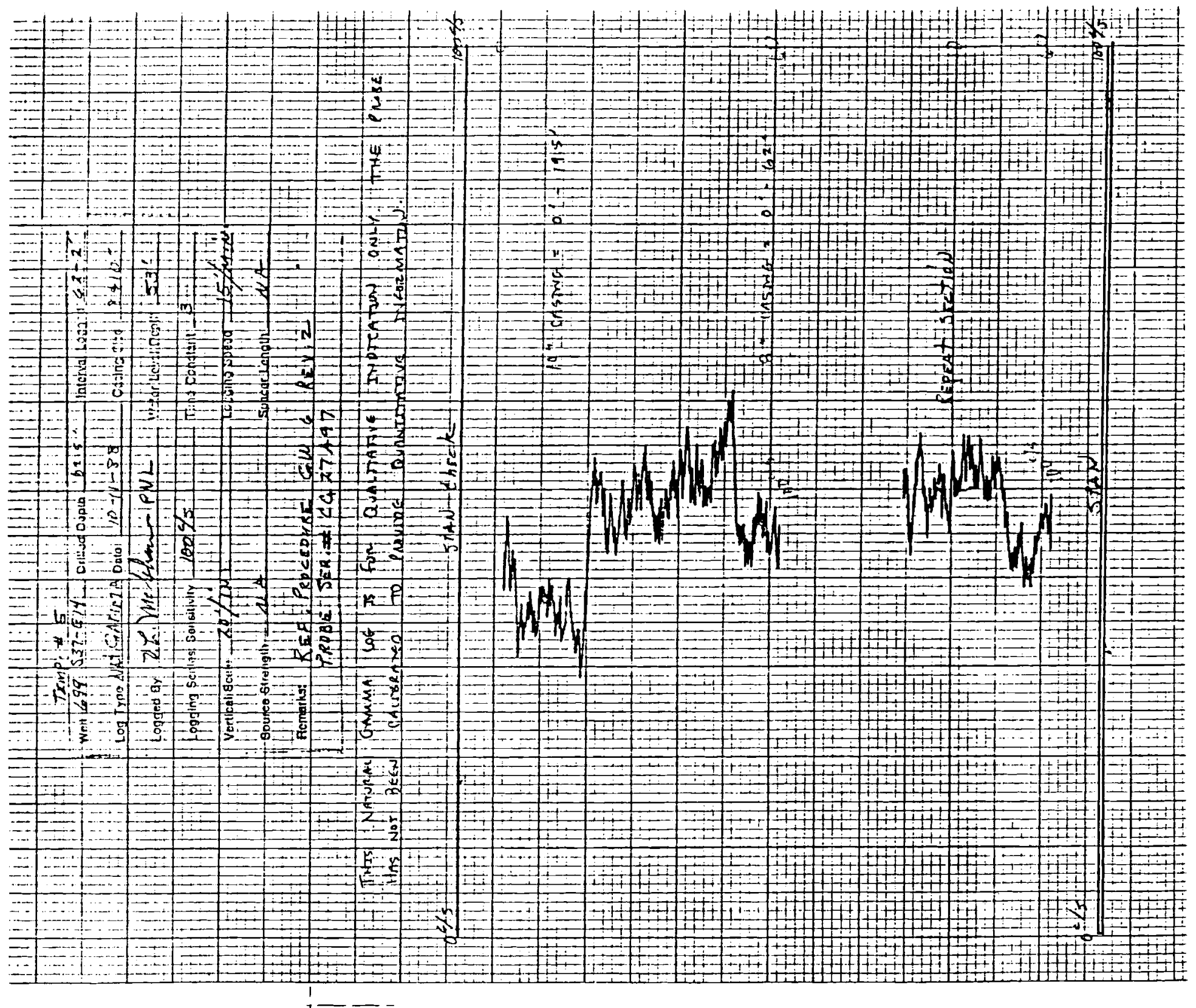




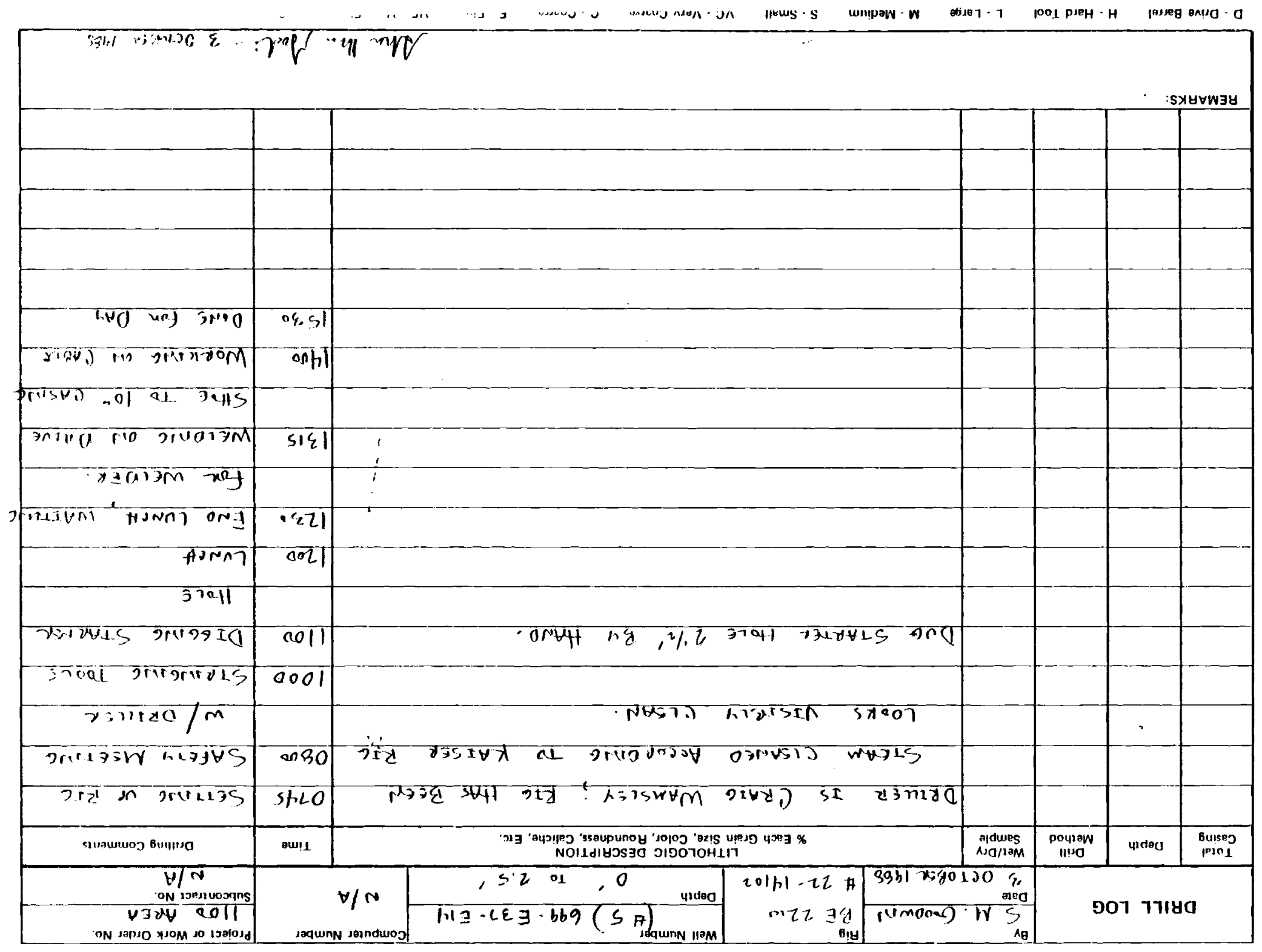


1 of 2

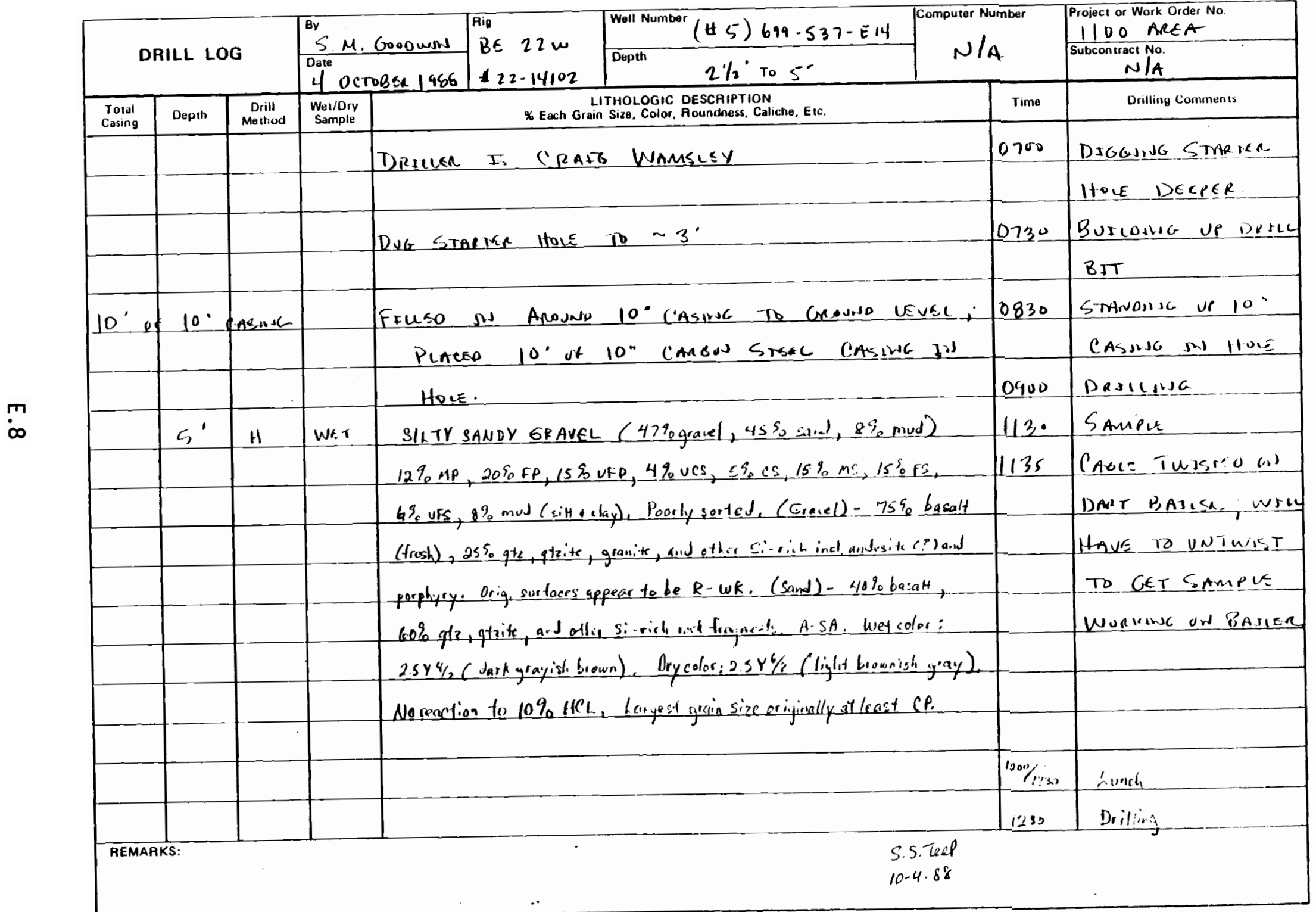

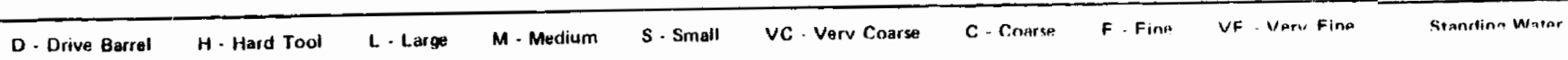




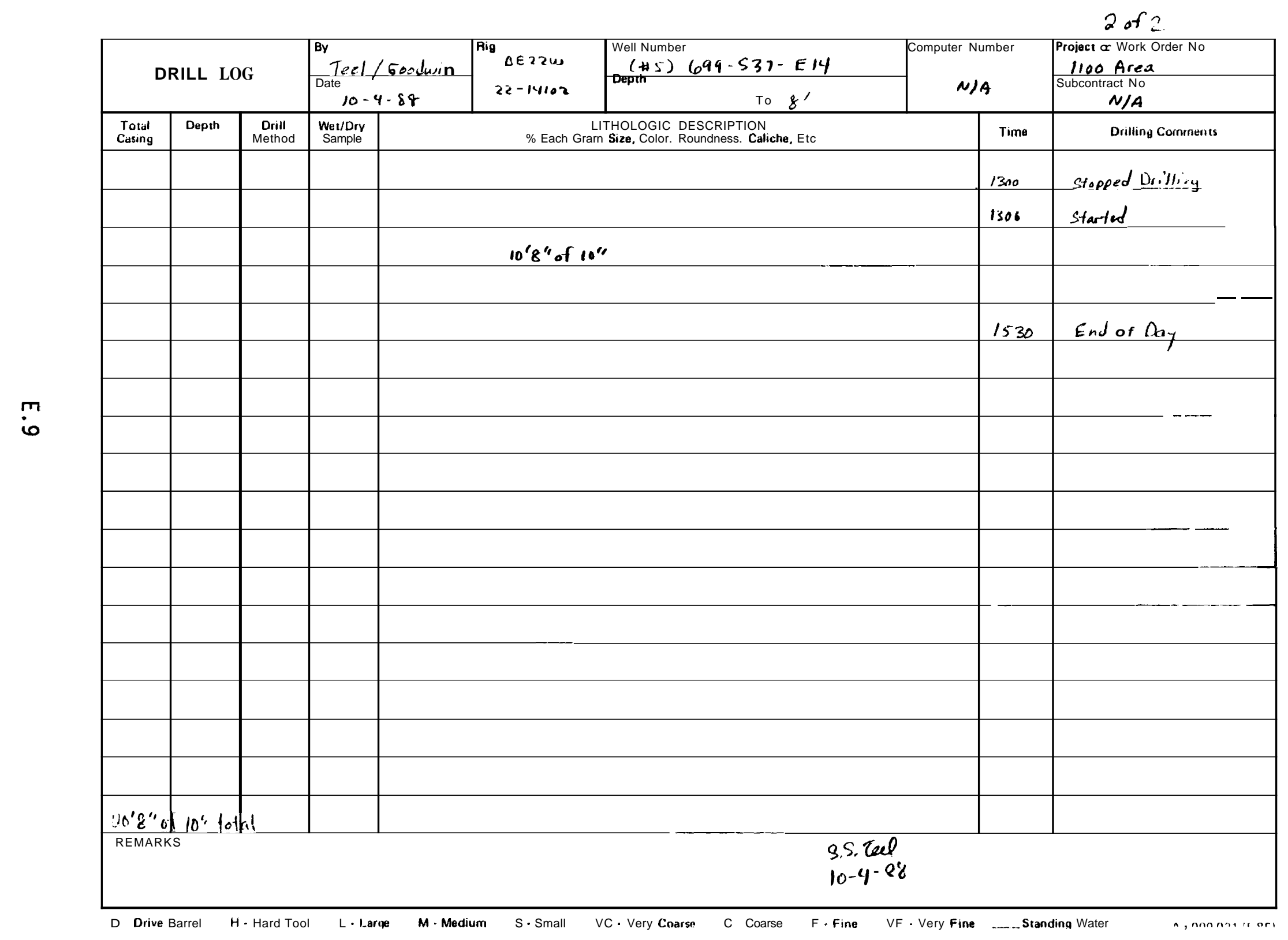




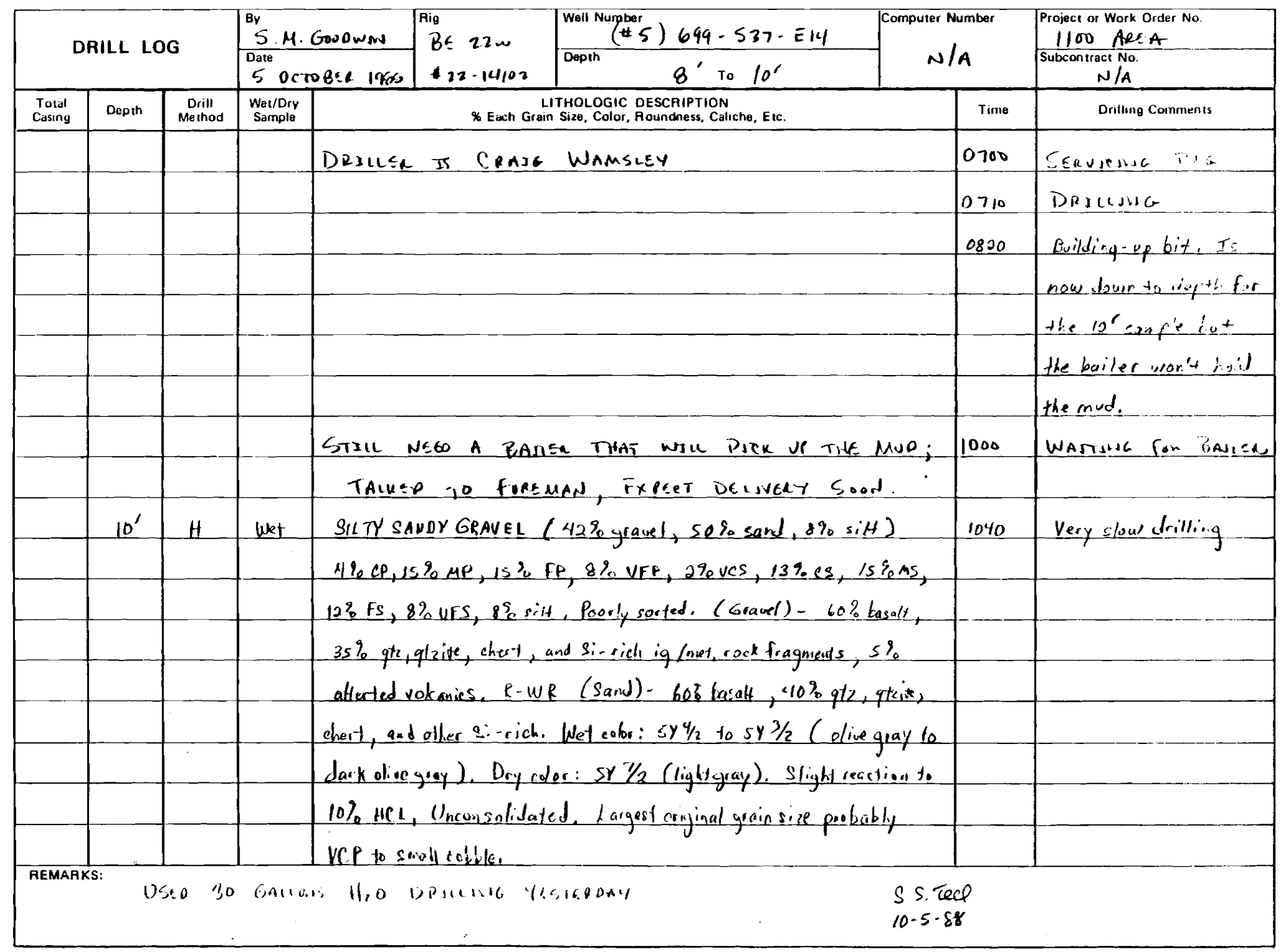

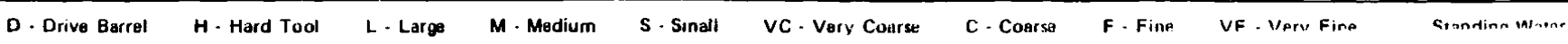


2 of 3

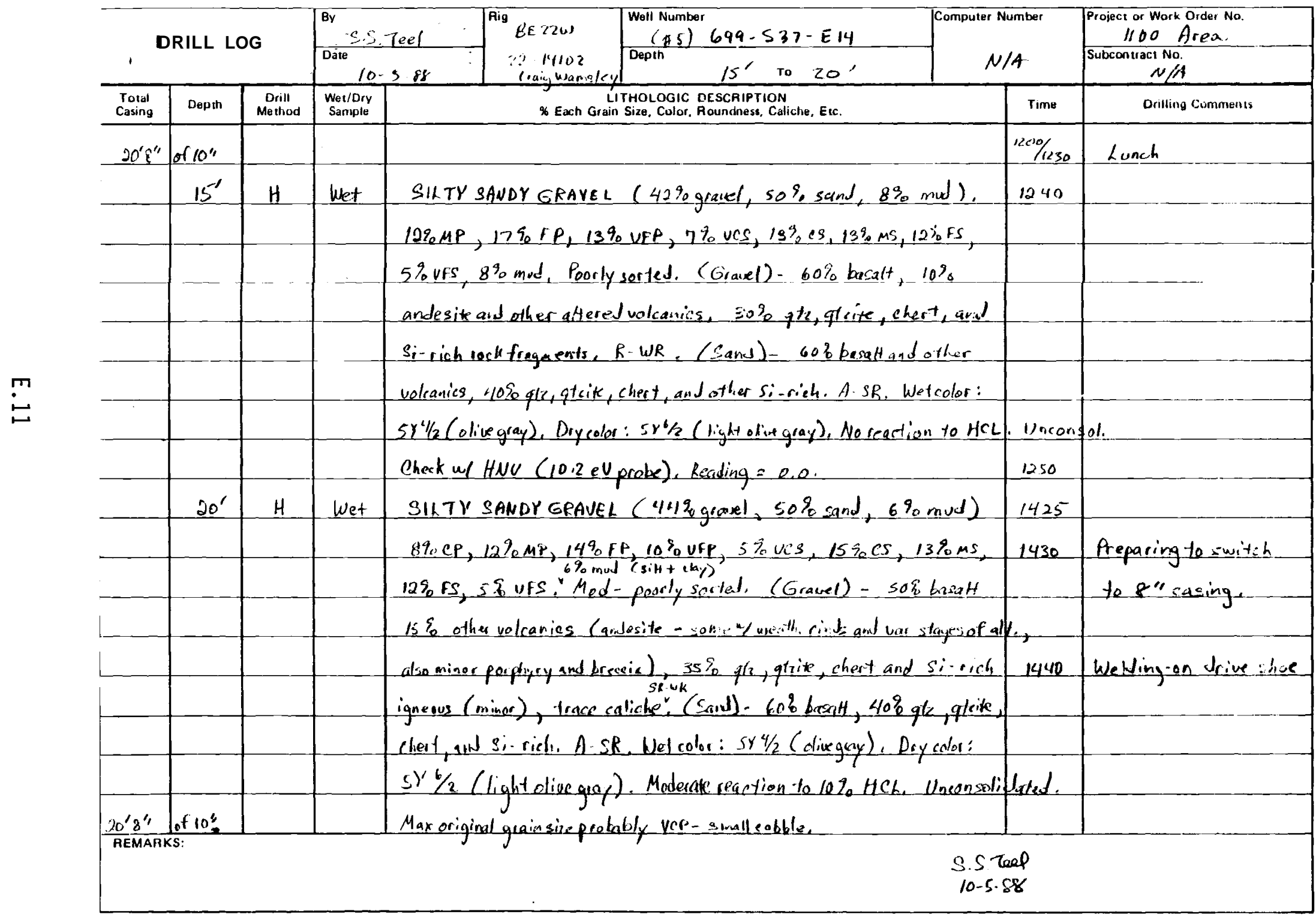

D-Drive Barrel H-Hard Tool L-Large M-Medium S. Small VC-Very Coorse C-Coarse F- Fina VF-Very Fine S t a $n$ d ing Water 


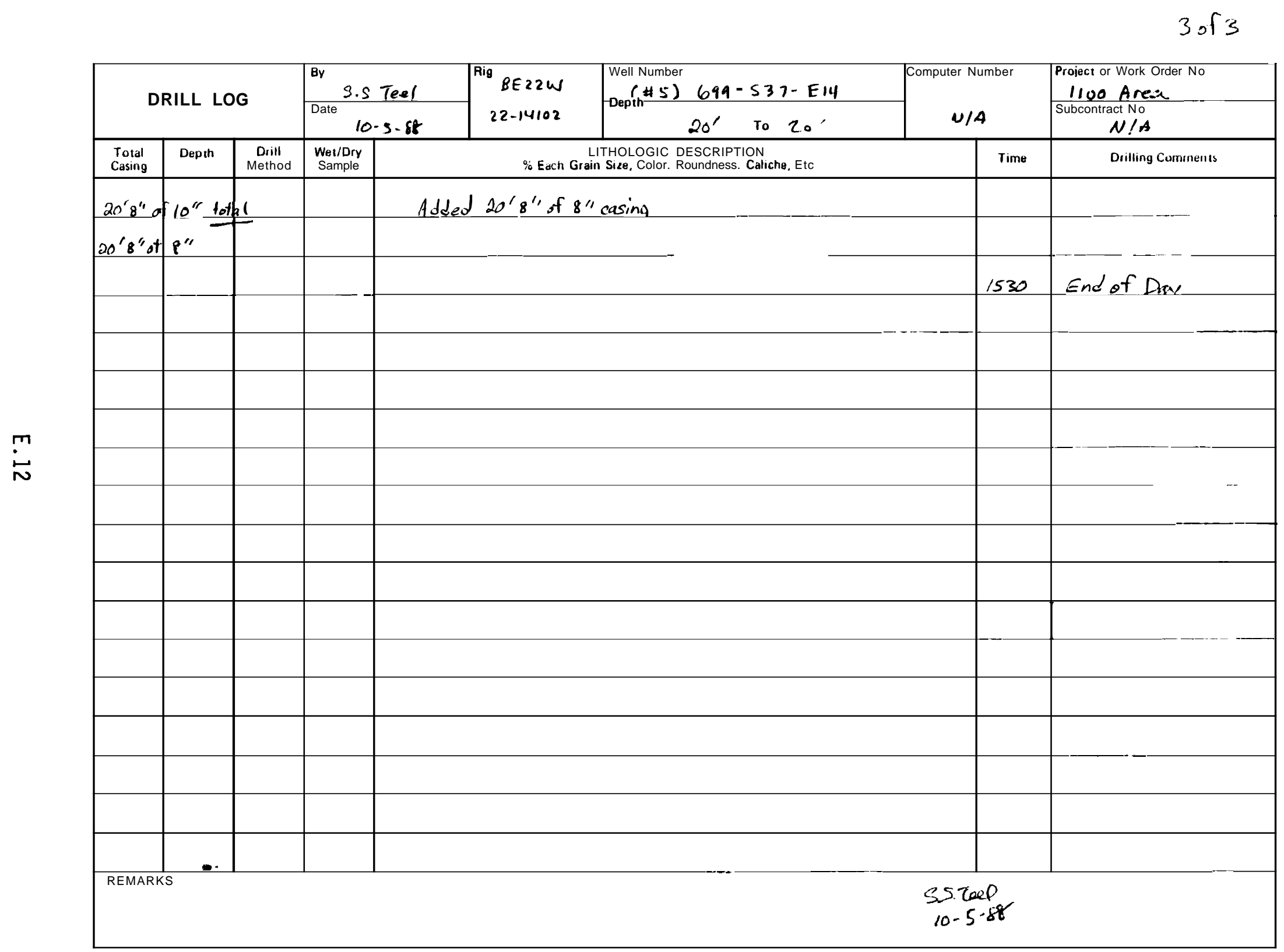

D. Drive Barrel H. Hard Tool L. Large $\quad$ M. Medium $\quad$ S. Small $\quad$ VC. Very Coarse $\quad$ C. Coarse $\quad$ F. Fine $\quad$ VF - Verv Fine Standina Warer 
1 of 4

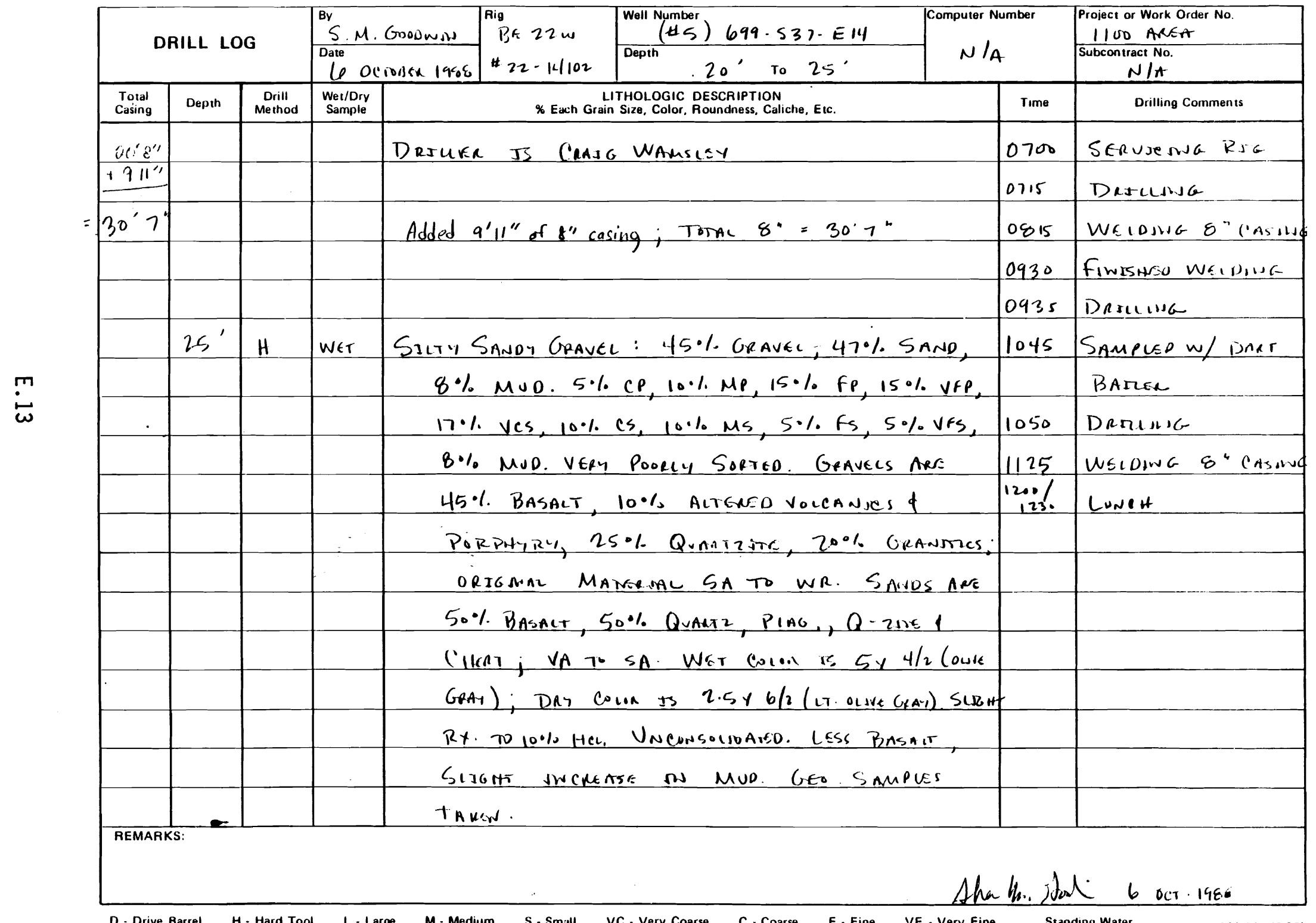


2 of 4

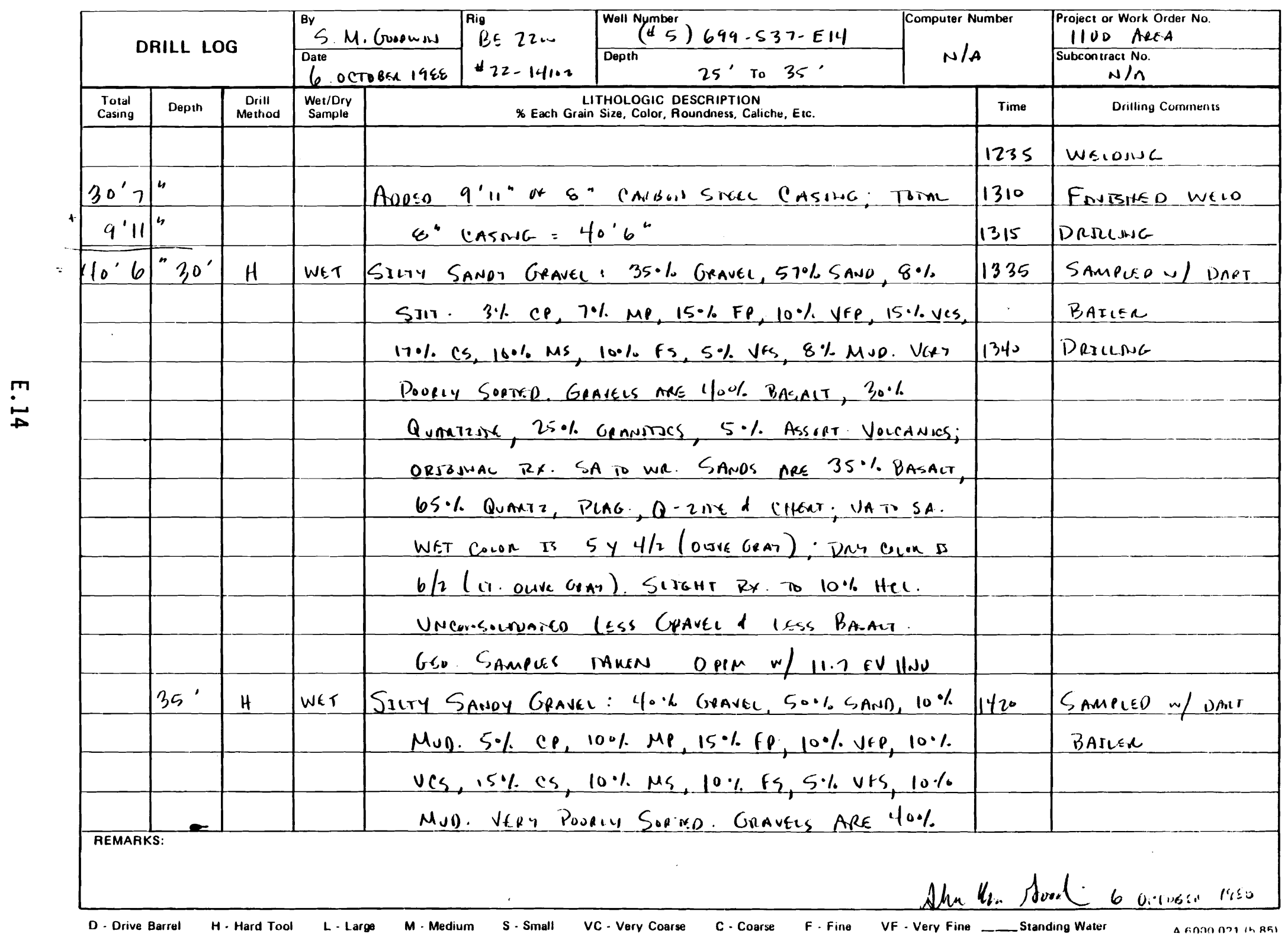


3 of 4

\begin{tabular}{|c|c|c|c|c|c|c|}
\hline \multirow{2}{*}{\multicolumn{3}{|c|}{ DRILL LOG }} & \multirow{2}{*}{ 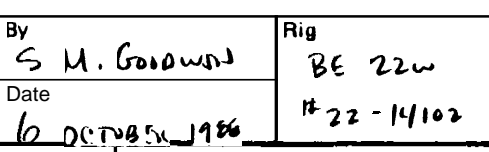 } & \multirow{2}{*}{\multicolumn{2}{|c|}{ 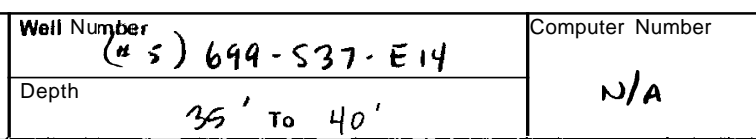 }} & \multirow{3}{*}{$\begin{array}{l}\text { Project or Work Order No } \\
11 \text { So AXEA } \\
\text { Subcontract No } \\
\text { N/A } \\
\text { Dilling Comments }\end{array}$} \\
\hline & & & & & & \\
\hline $\begin{array}{c}\text { Total } \\
\text { Casing }\end{array}$ & Depth & $\begin{array}{c}\text { Drill } \\
\text { Method }\end{array}$ & $\begin{array}{l}\text { Wet/Dry } \\
\text { Sarnple }\end{array}$ & $\begin{array}{l}\text { LITHOLOGIC DESCRIPTION } \\
\text { * Each Grain Size, Color, Roundness, Caliche, Etc }\end{array}$ & Trme & \\
\hline & $35^{\prime}$ & $H$ & WeT & (cont.) Basalt, $5 \cdot 1$, Aitseg) Vocconser d Ponpitual, & 1425 & Dasulins \\
\hline & & & & 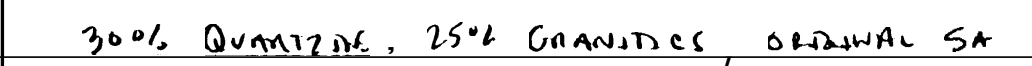 & & \\
\hline & & & & WR SANDS $35 \%$. BASACT, $65 . \%$ QUAMIT. & & \\
\hline & & & & PlaG, CHENT, $Q$ - 2JKE: VA TO SA. No Rr, TP & & \\
\hline & & & & 100\% Hel. WET Color ix $5 y+11$ (D)rak Crany): & & \\
\hline & & & & 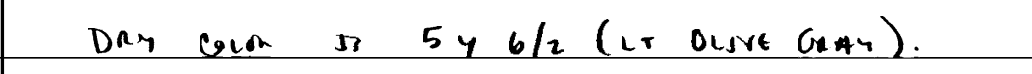 & & \\
\hline & & & & 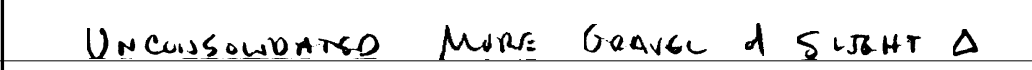 & & \\
\hline & & & & 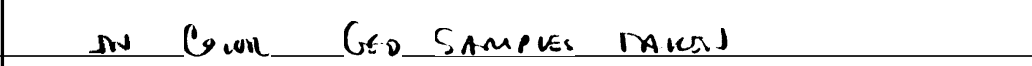 & & \\
\hline & $4 \cdot 1$ & $\mathrm{H}$ & WET & GraveuY SAND $15 \%$ Gravel, $77 \%$ SANO, $8 \%$ Mud. & 1505 & Sampuso w/DAnT \\
\hline & & & & $1 \%$ cp, $3 \%$ mp, $6 \%$ fe, $5 \%$ vep, $15 \%$ ves, $30 \%$ cs. & & BAREA \\
\hline & & & & 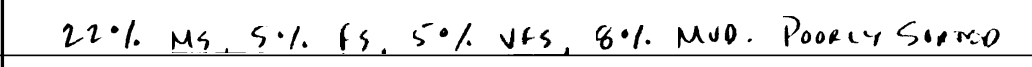 & 1510 & QuiT Darunha \\
\hline & & & & Gravels ne 40\% Basalt; 10\% ALtTEnso vilcavics 1 & & Clsonuik up \\
\hline & & & & ; $25 \%$ Goanmes $25 \%$ QunMrzane, ORTGMal & 1525 & Done Gor \\
\hline & & & & R.Y SA TO WR SANOS ANRE 36.\% BASALT $6 \%$ AINANS) & 1530 & RPT SURUEY, No \\
\hline & & & & 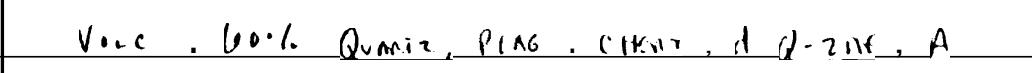 & & DET Conings- \\
\hline & & & & WCA. Wer Cacoa is $5.1 \mathrm{1} / 2$ (Dikk ocive (JoA. ), & & Atros \\
\hline & & & & 1) Bay Coion is $546 / 2 / 27$ OcNeG Gay) No & & $w /$ thd oft stos \\
\hline & & & & Obriods kx $10 \quad 10 \%$ C & & $d n$ Hucs. \\
\hline REMAR & & & & & She & 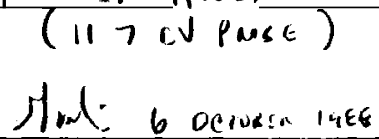 \\
\hline
\end{tabular}


4.5

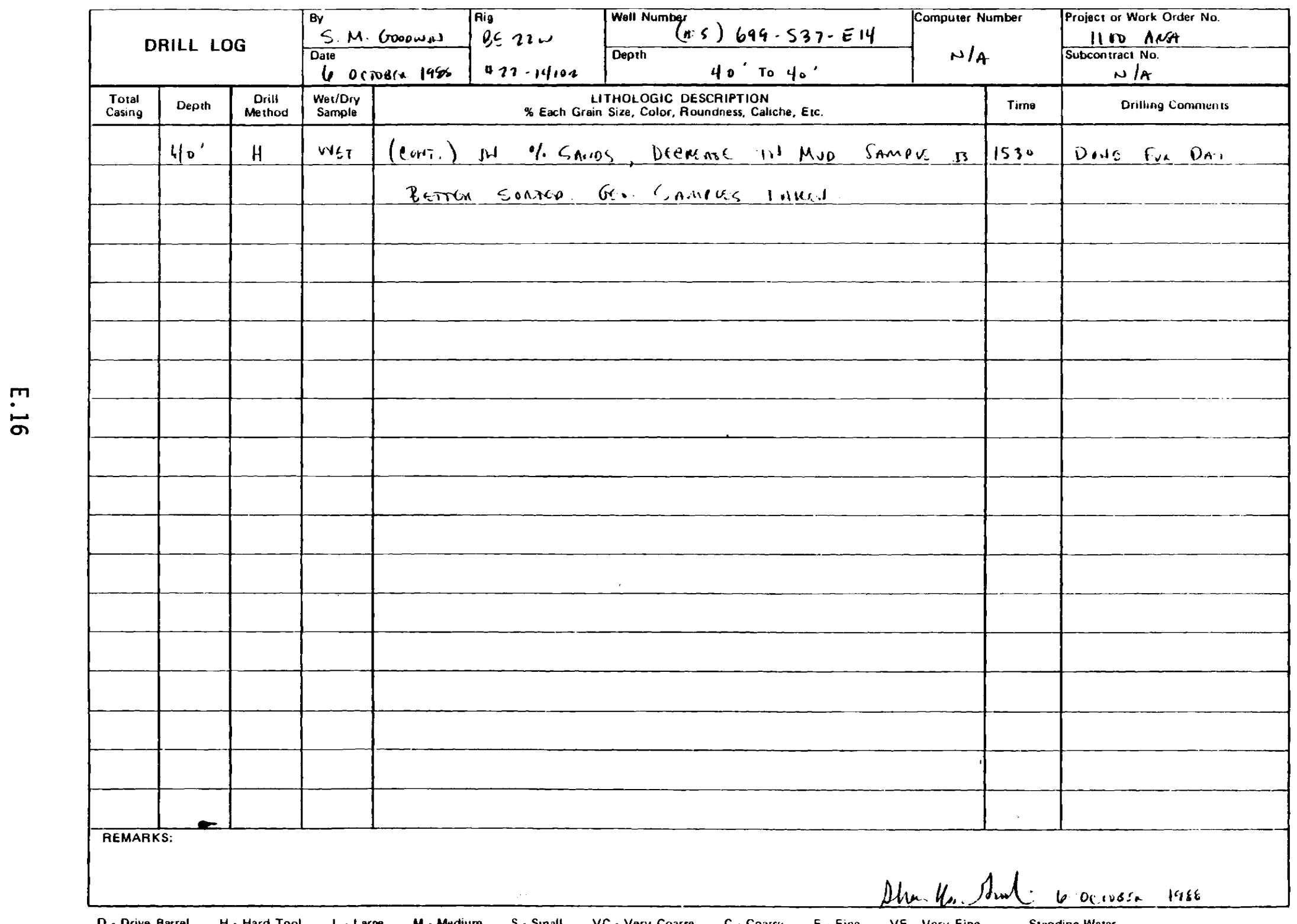

D. Drive Barrel H. Hard Tool L. Large M-Madium S-Sinall VC-Very Coarse C.Coarse F-Fine VF-Verv Fine _...Standinn Water 
1 o. 3

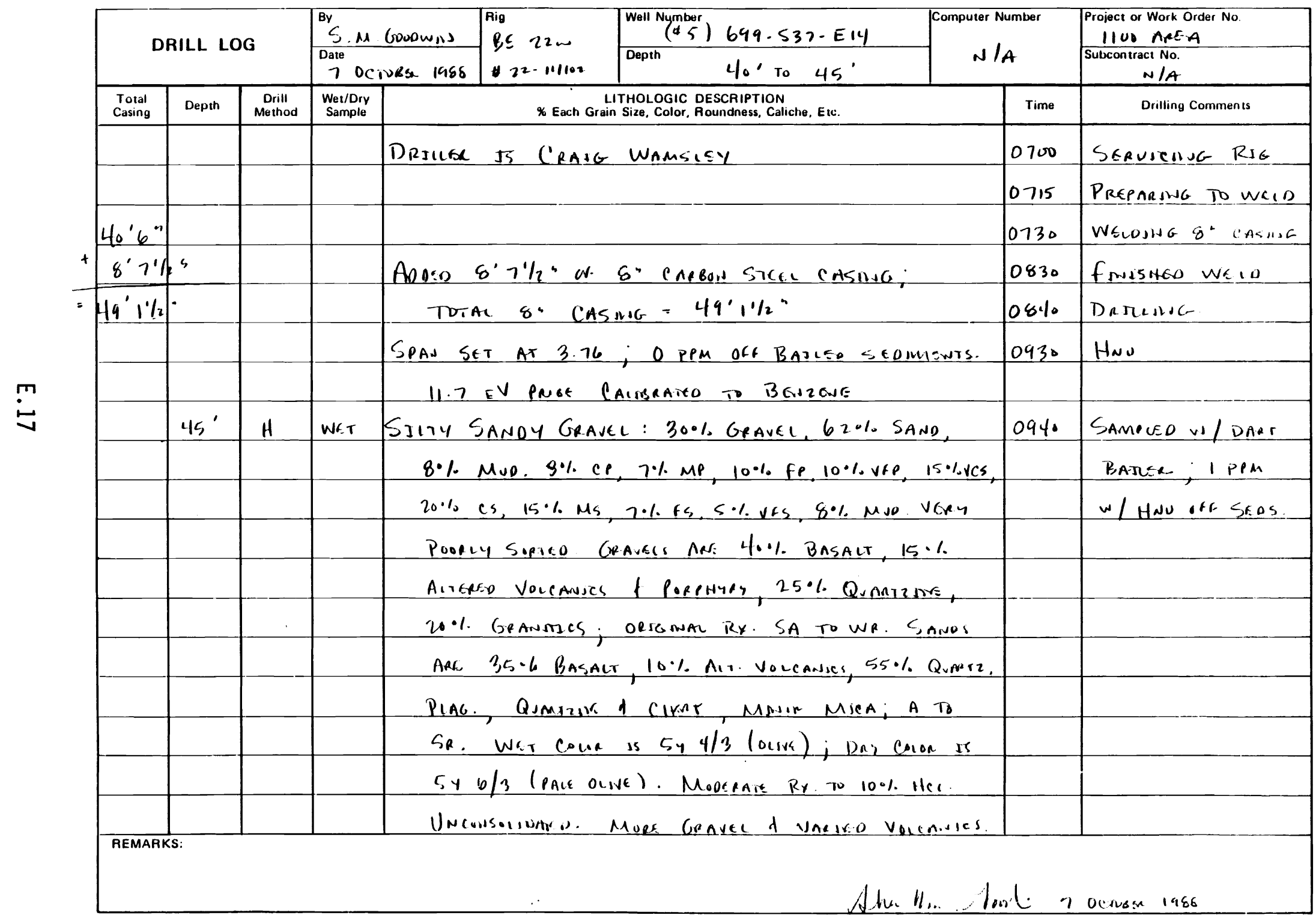

D. Drive Barrel H- Hard Tool L. Large M-Medium S-Small VC-Very Coarse $\quad$ C-Coarse F-Fine VF-Verv Fine ...... Standina Water 


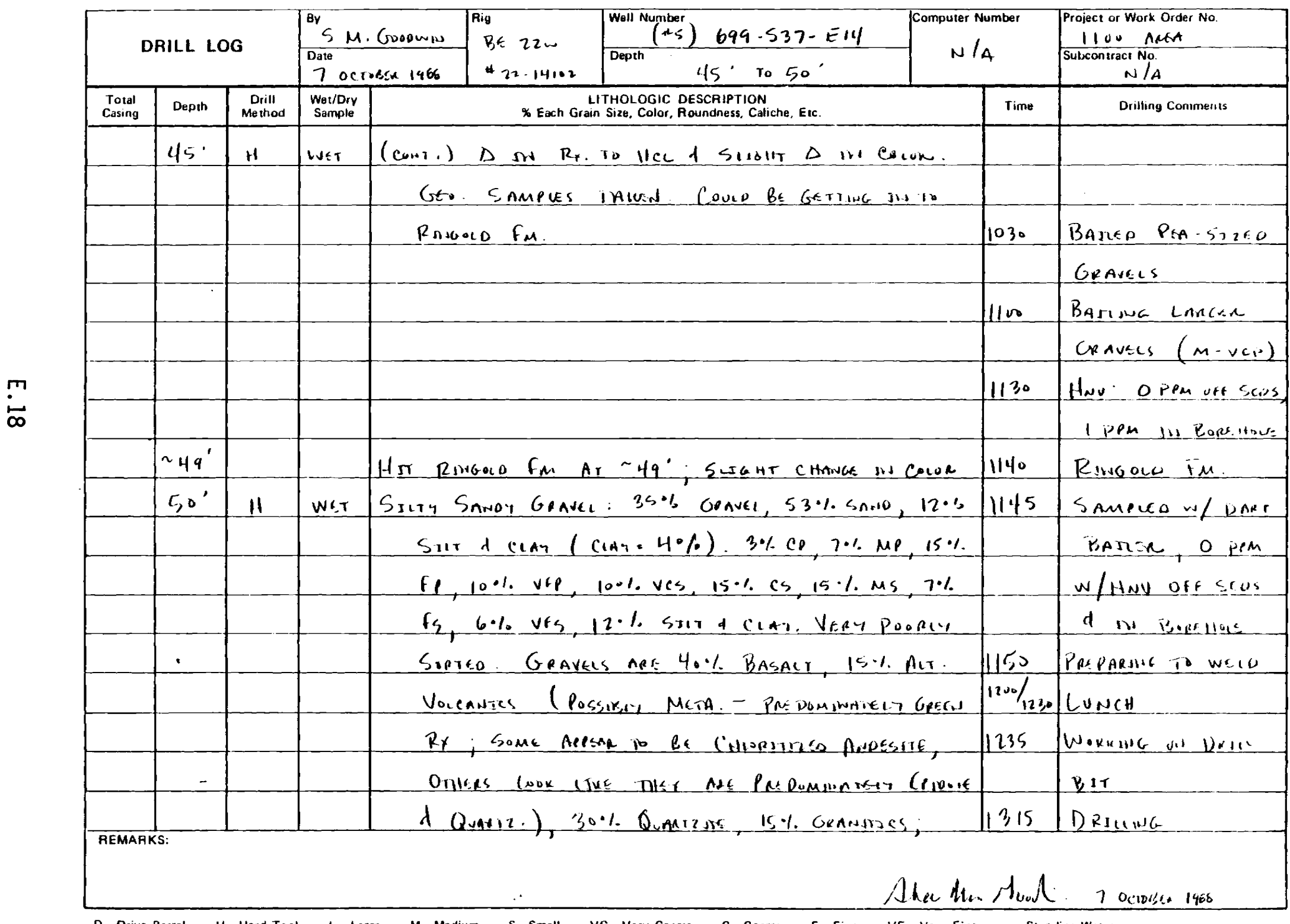

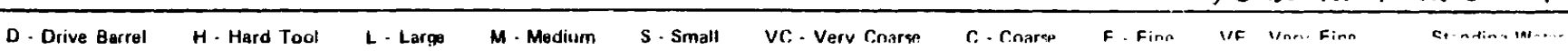




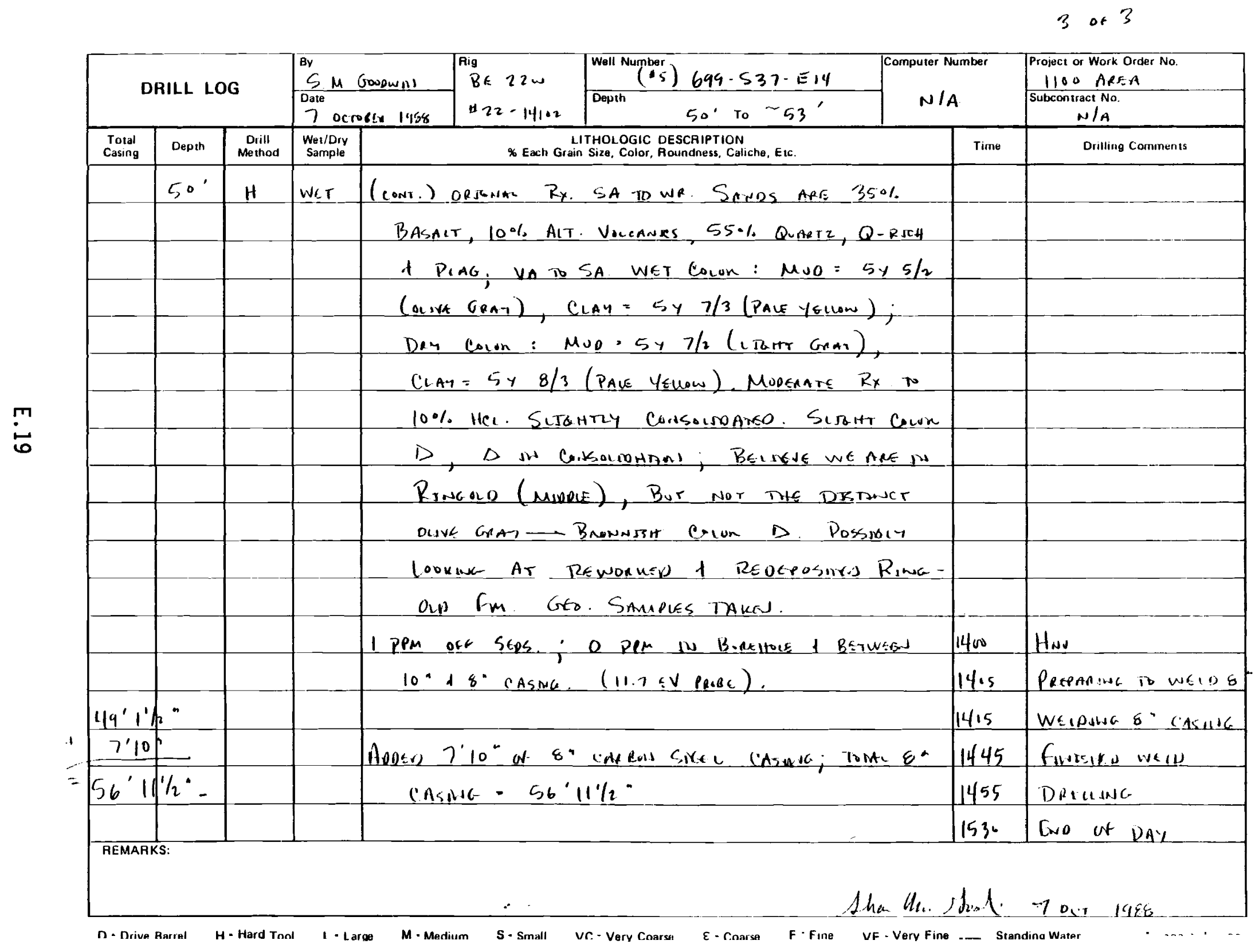


1 of 3

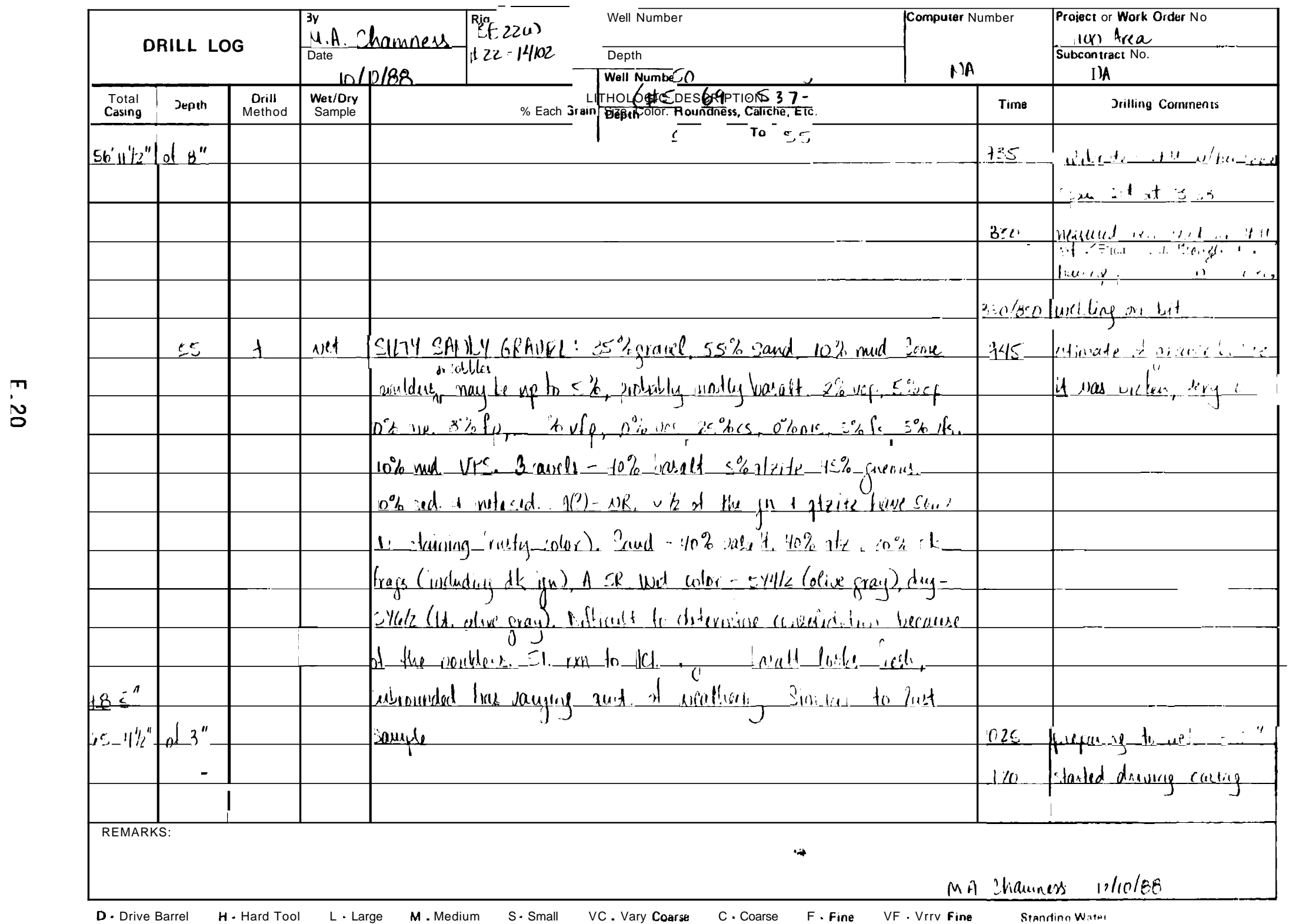


2 of 3

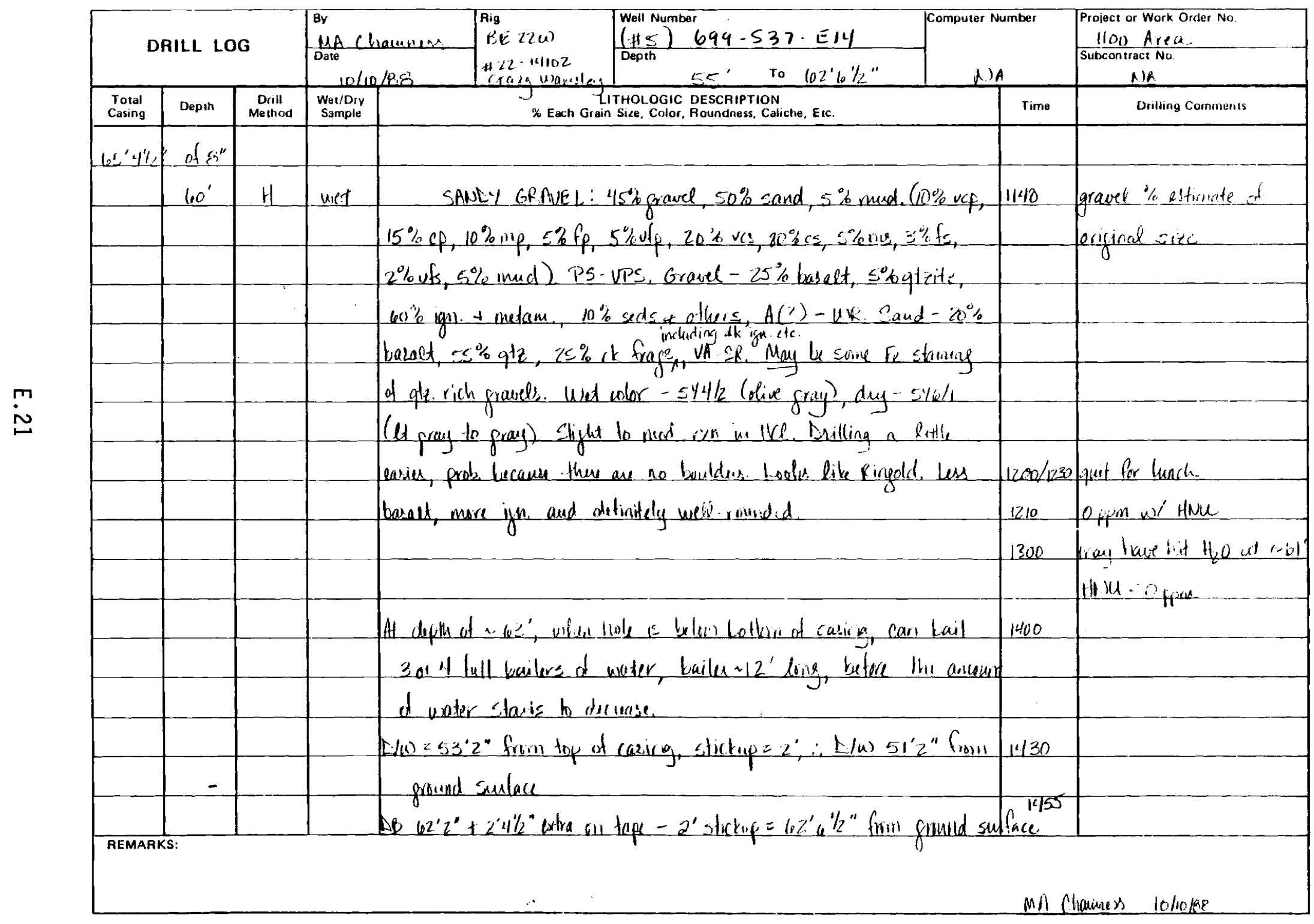

L. Large M-Medium

s. Small

VC - Verv Coarse

c. Comes

F Firn VE VMW E

r. ....... . 
3 of 3

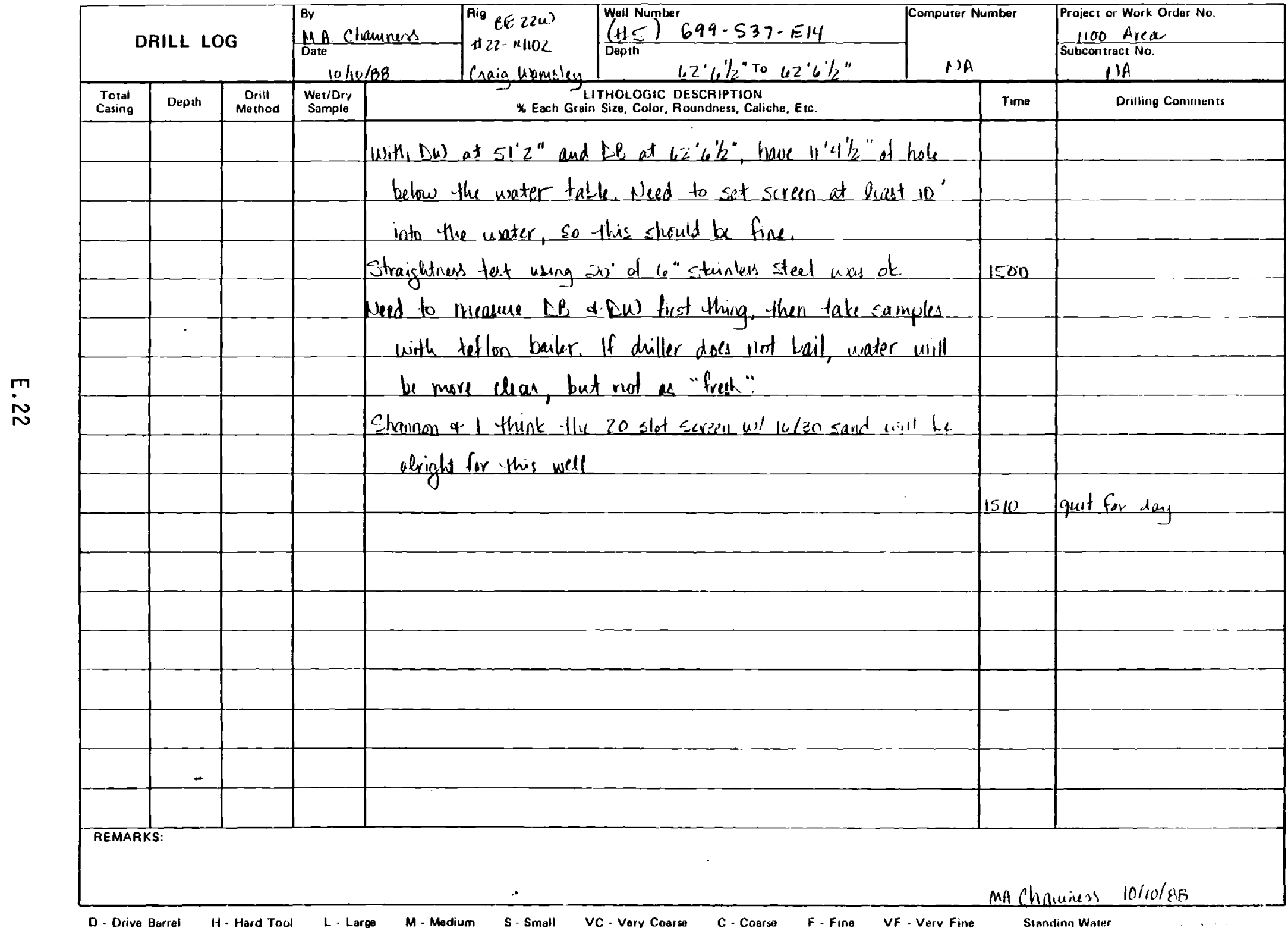


1 or 4

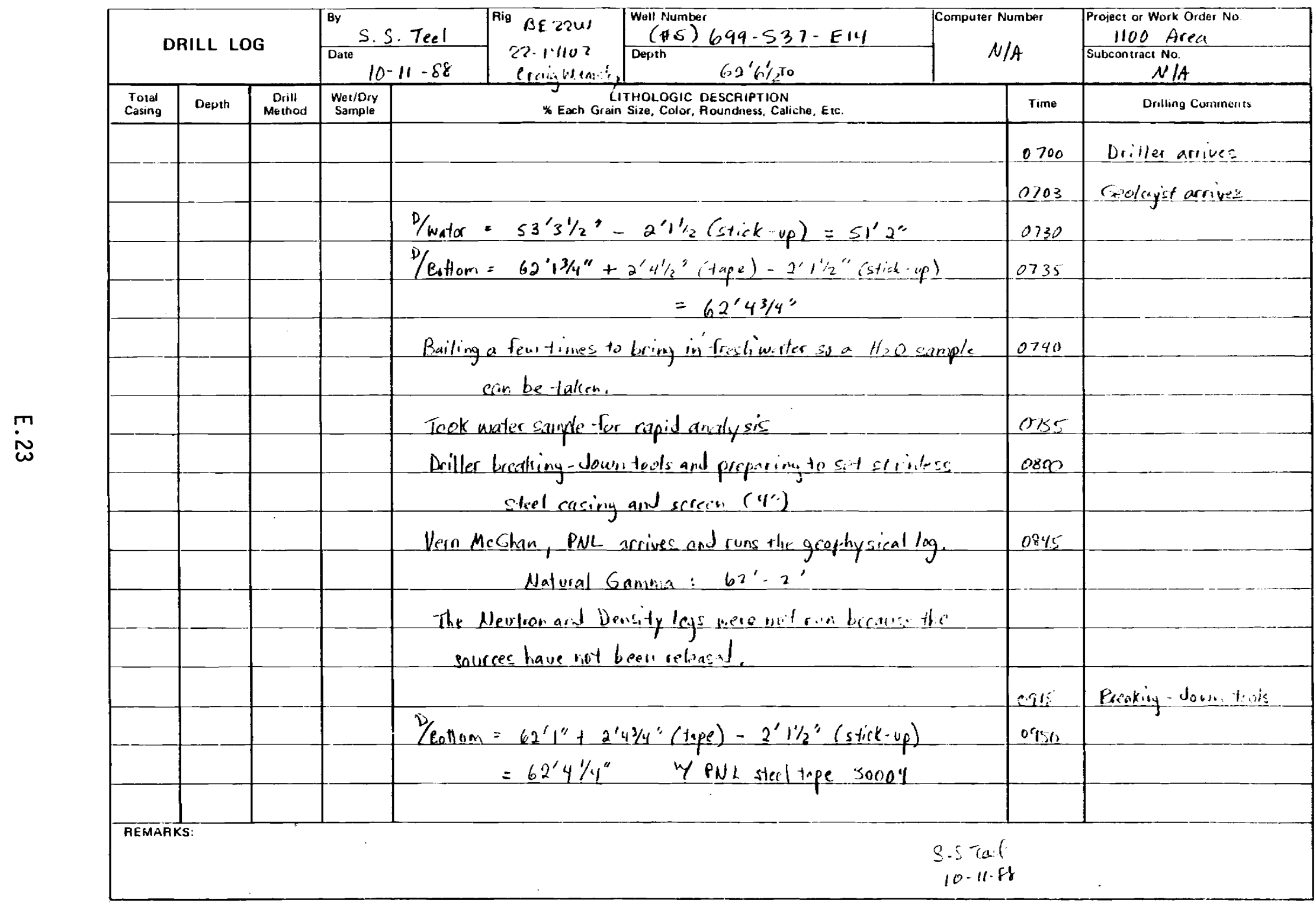

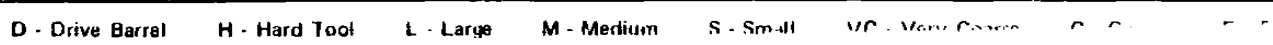




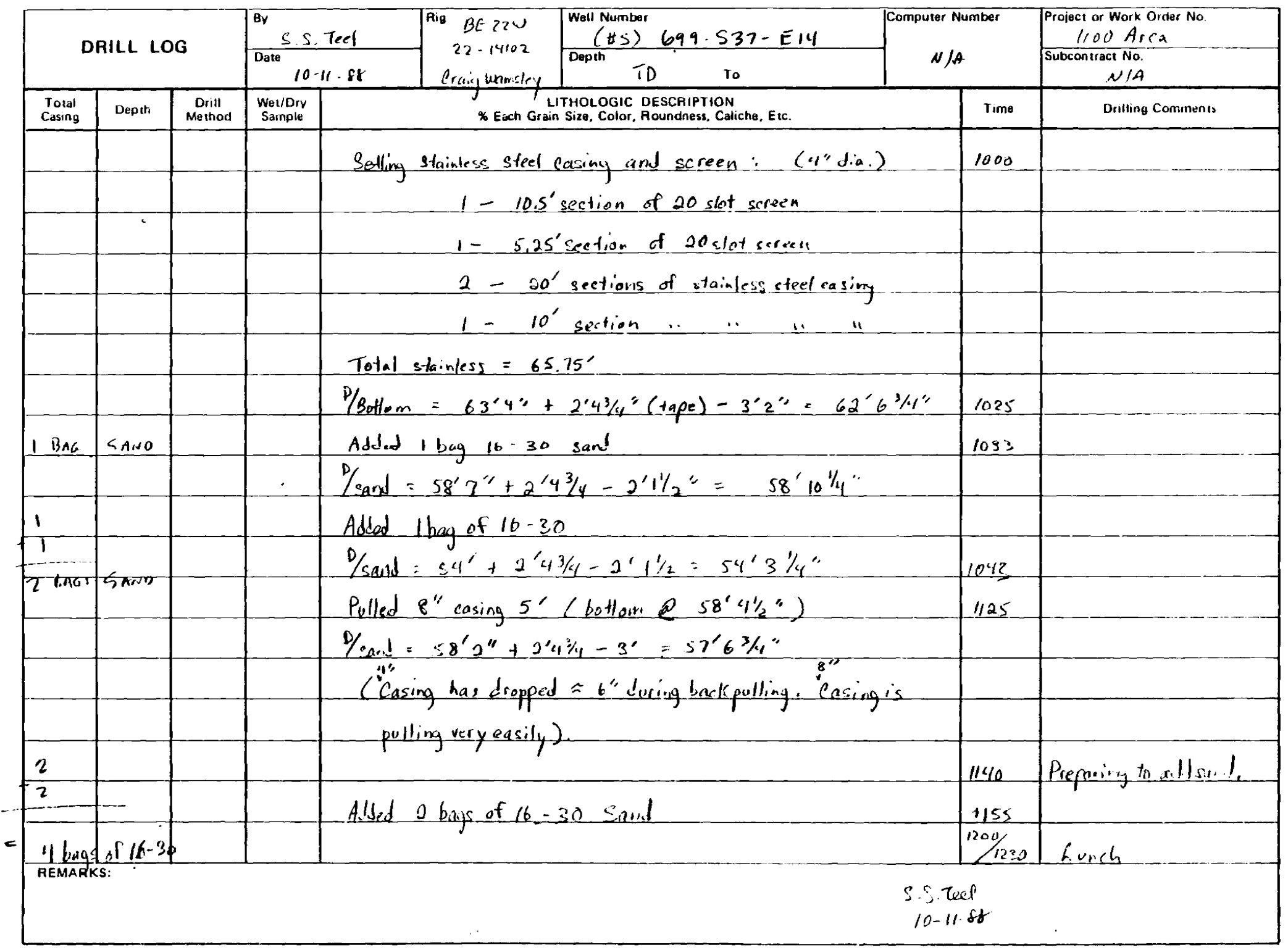

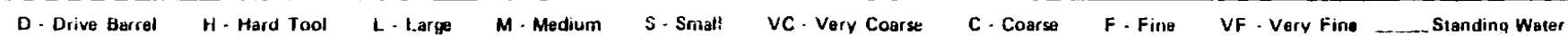




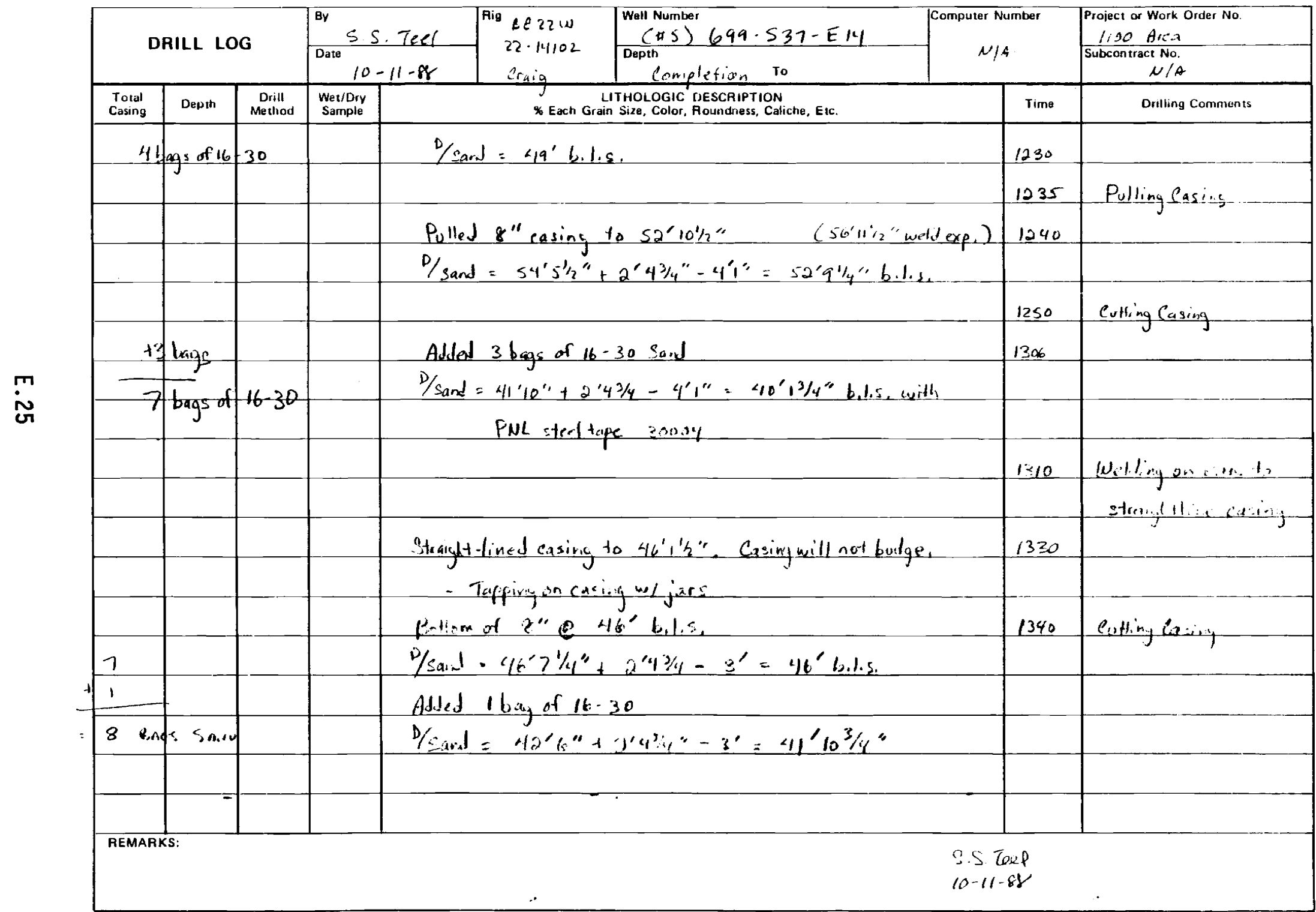




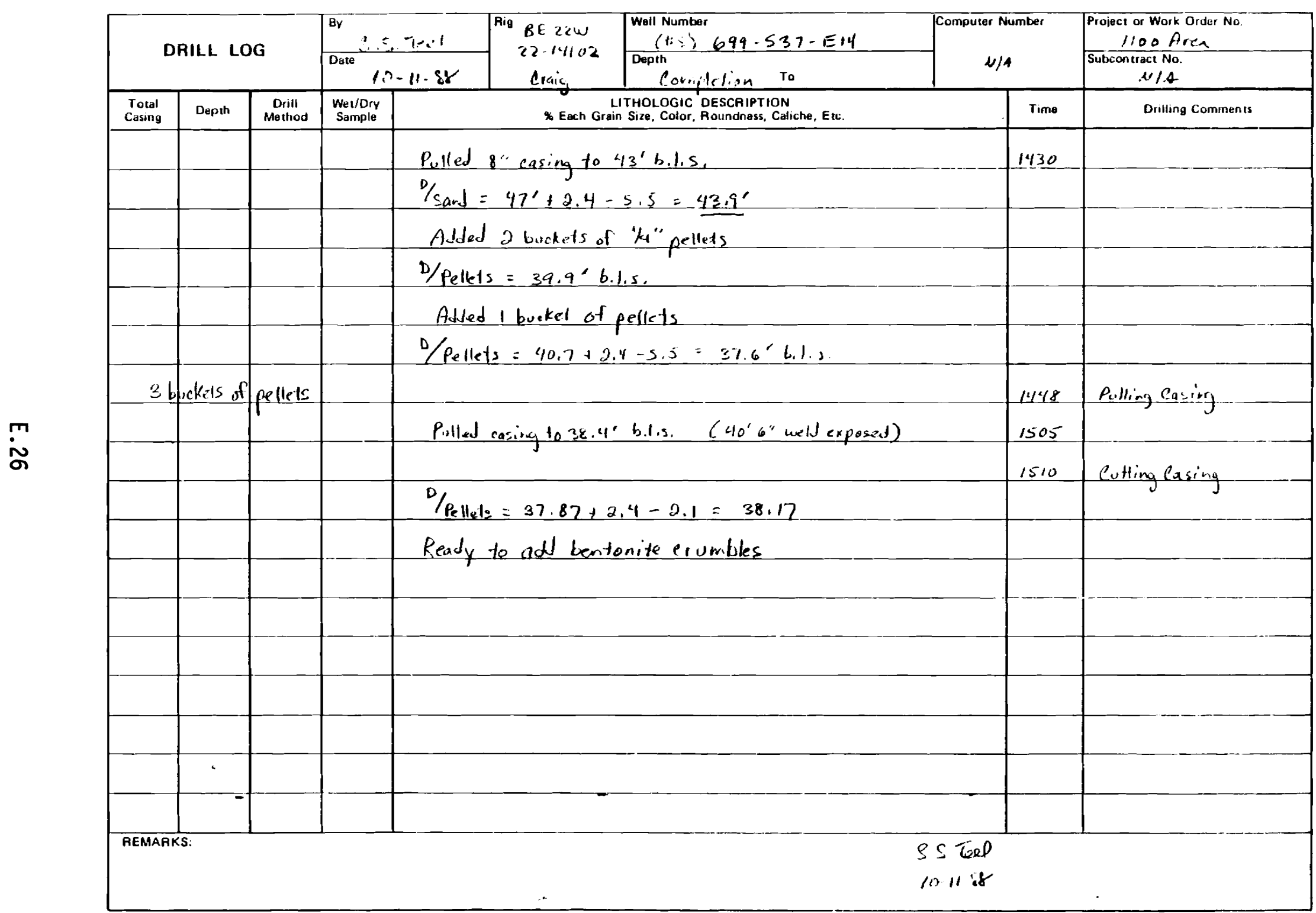

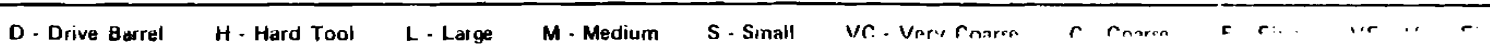


1 of 3

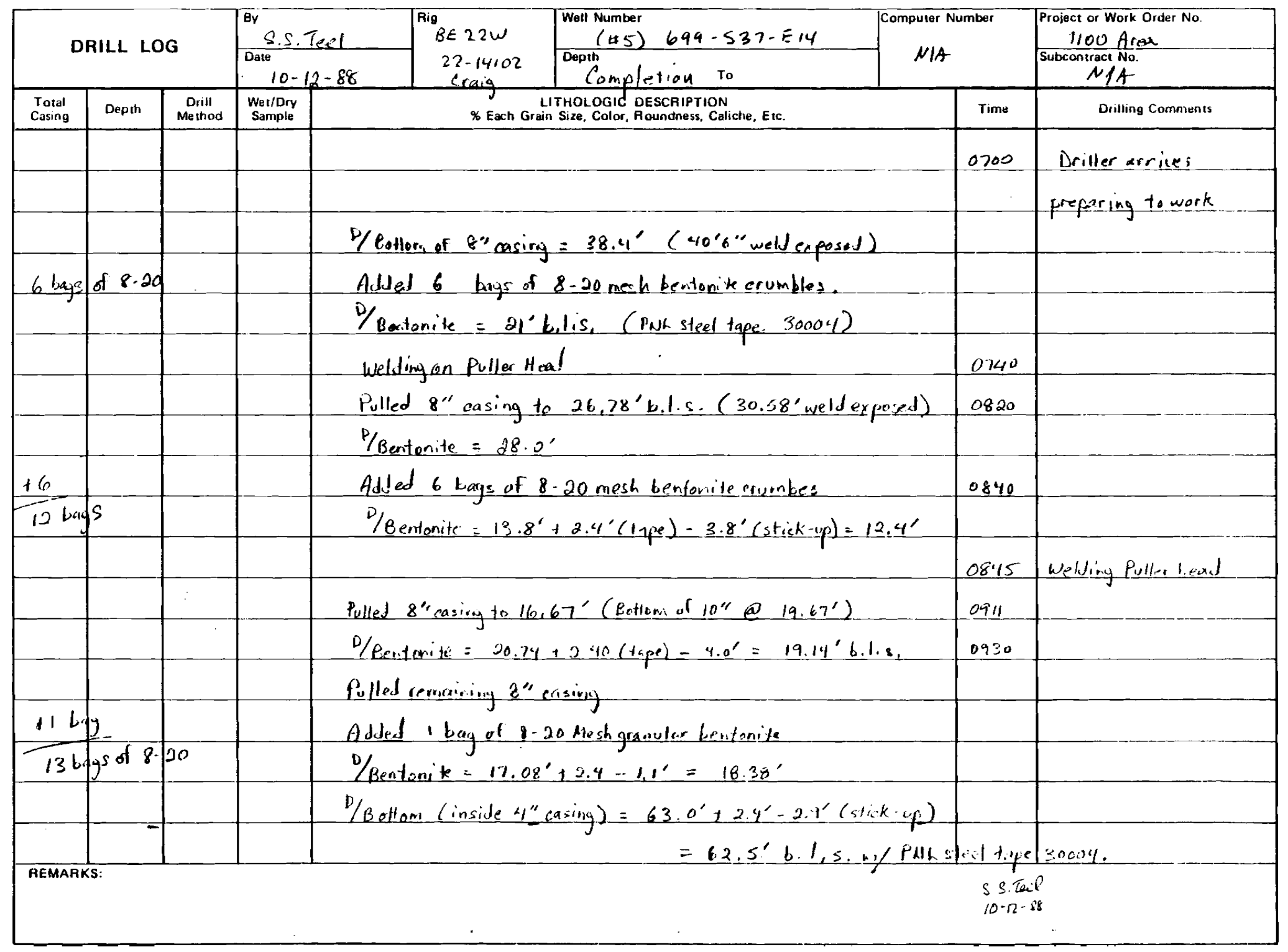




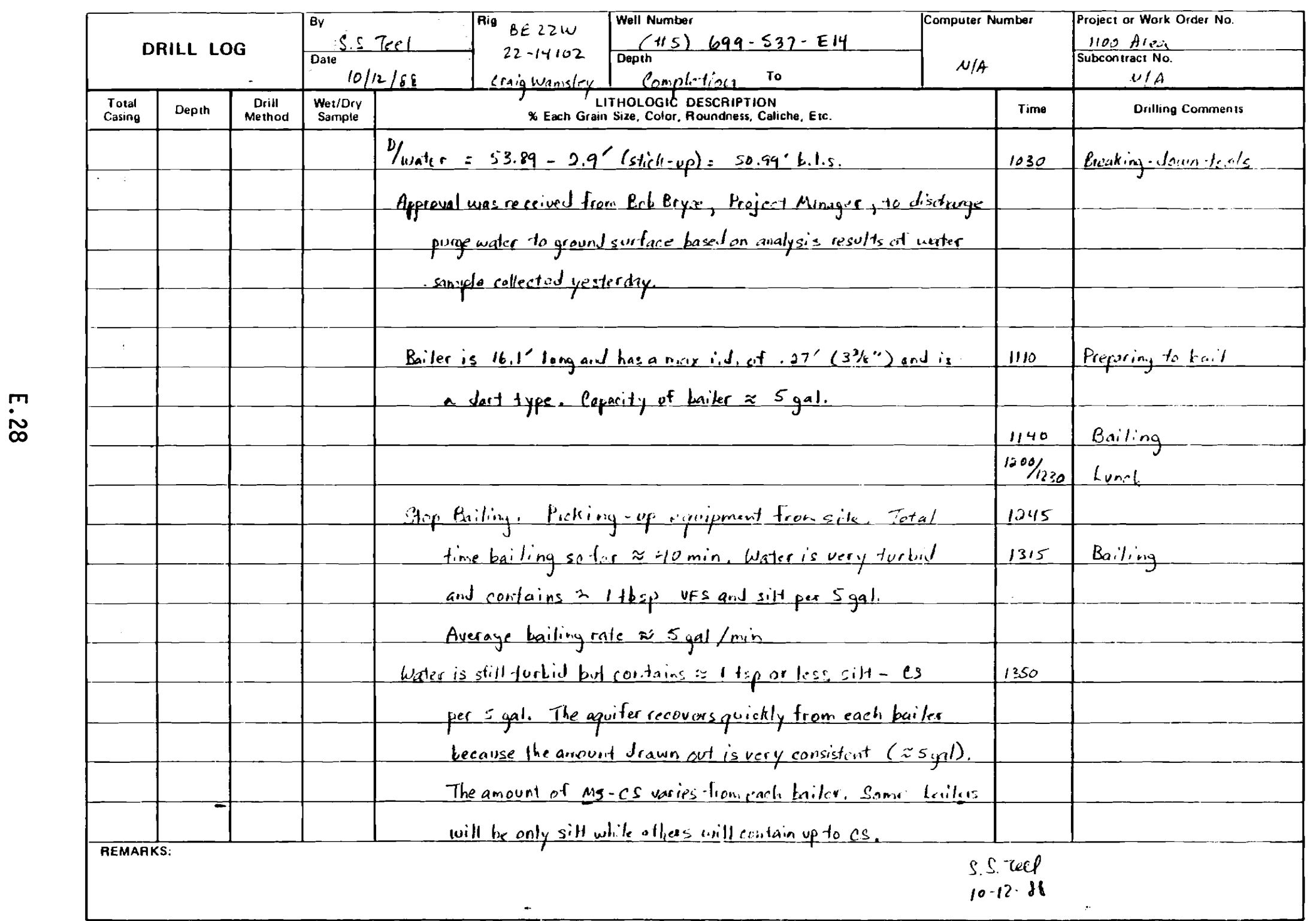

D. Drive Barrel H. Hard Tool L. Large M-Medium S-Small VC-Verv Caarse C - Coarse F-Fine VF-Very Fine .. Standinn Water 
3 of 3

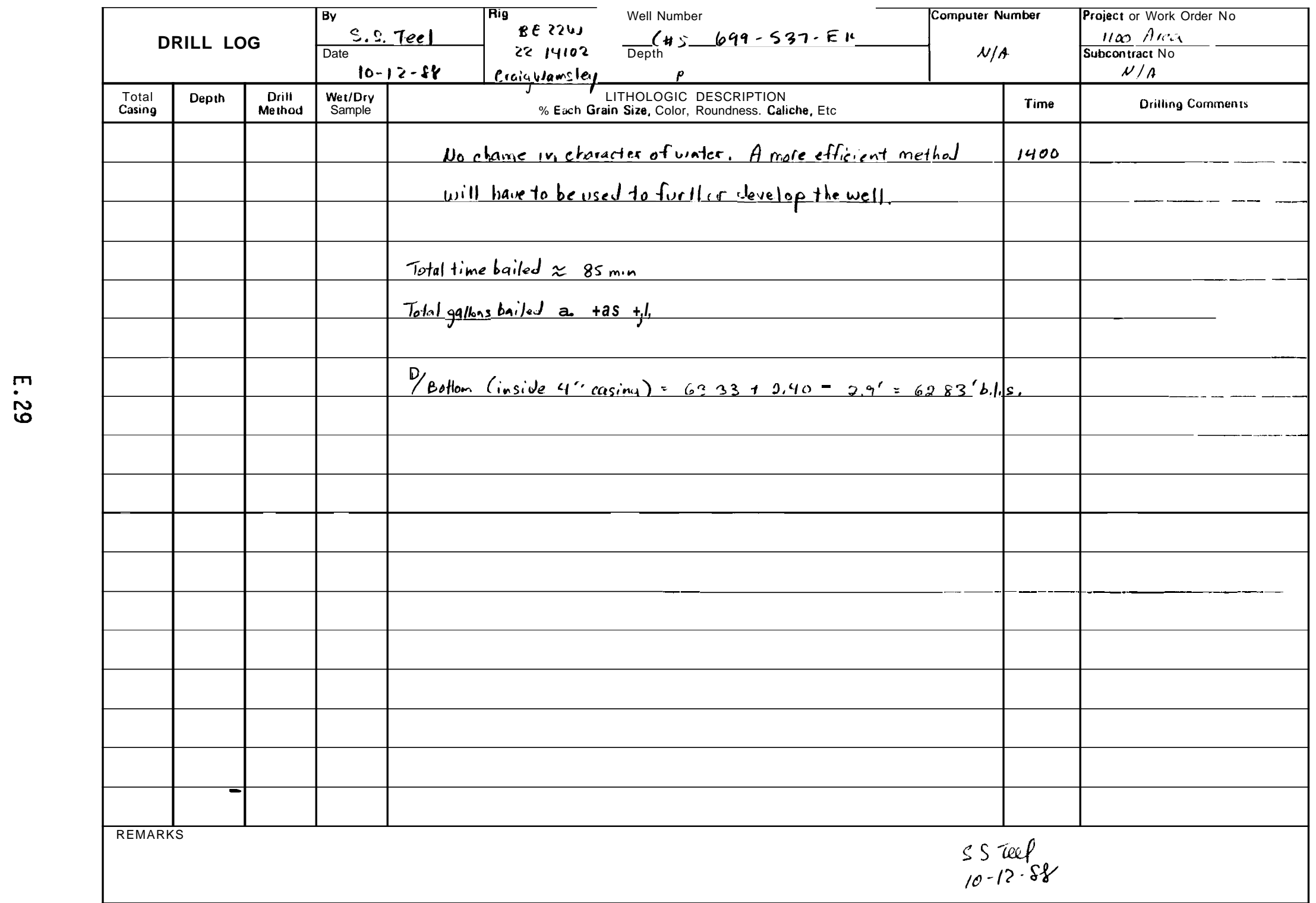

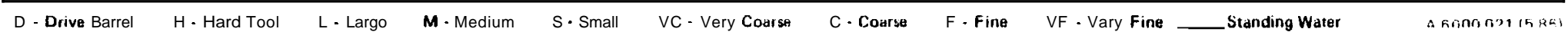


$10 f 1$

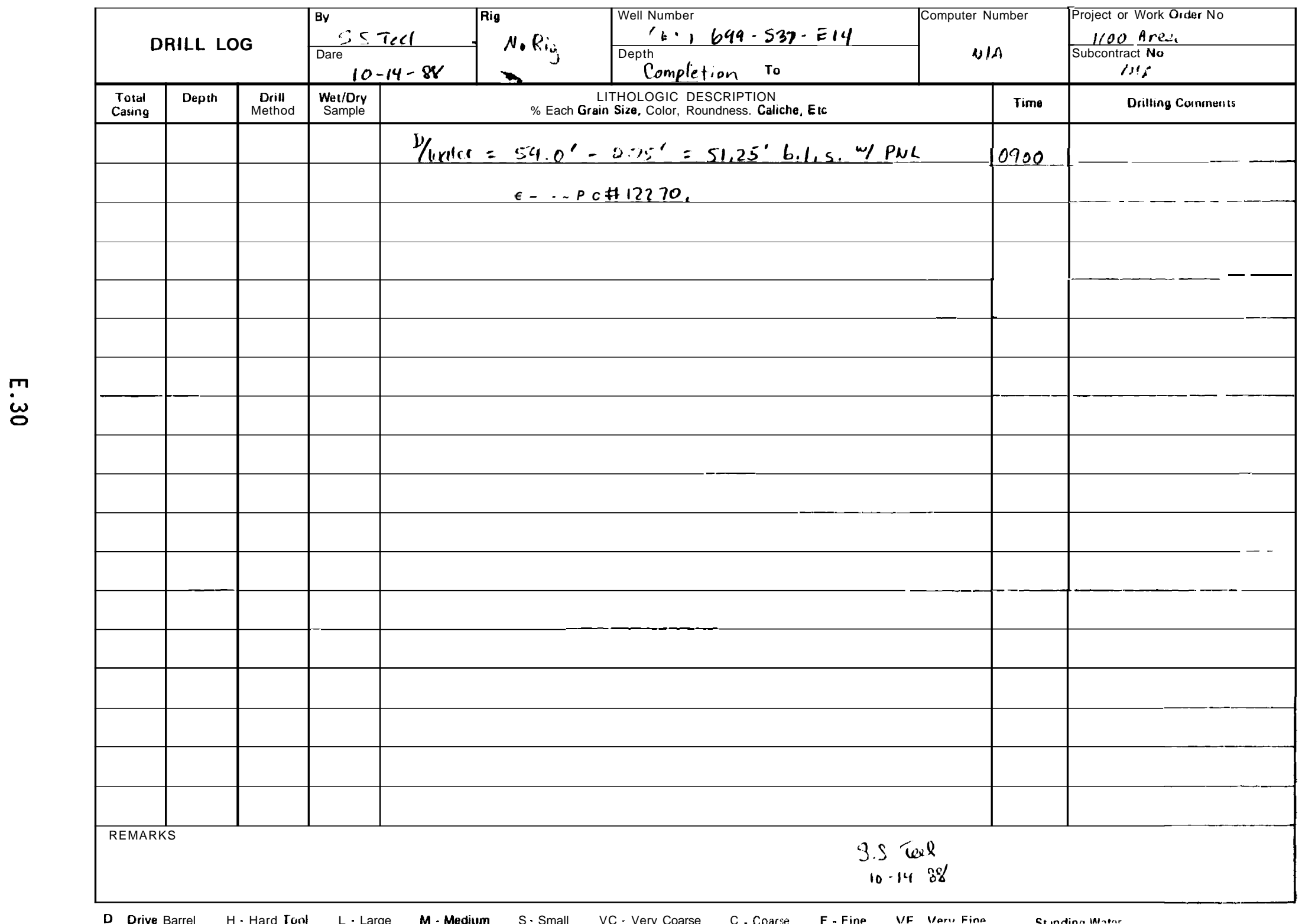


1 of

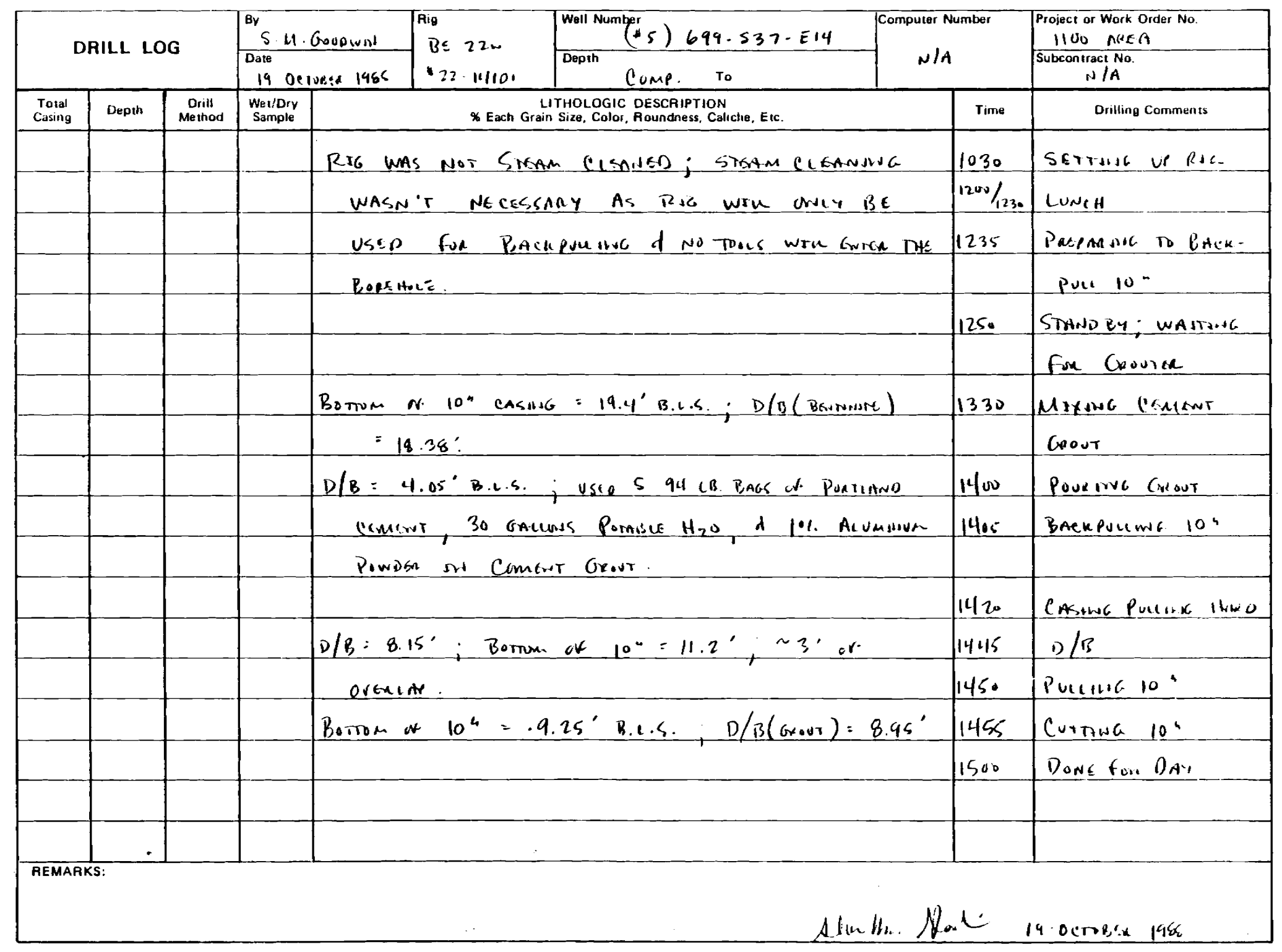

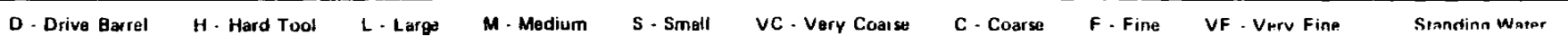




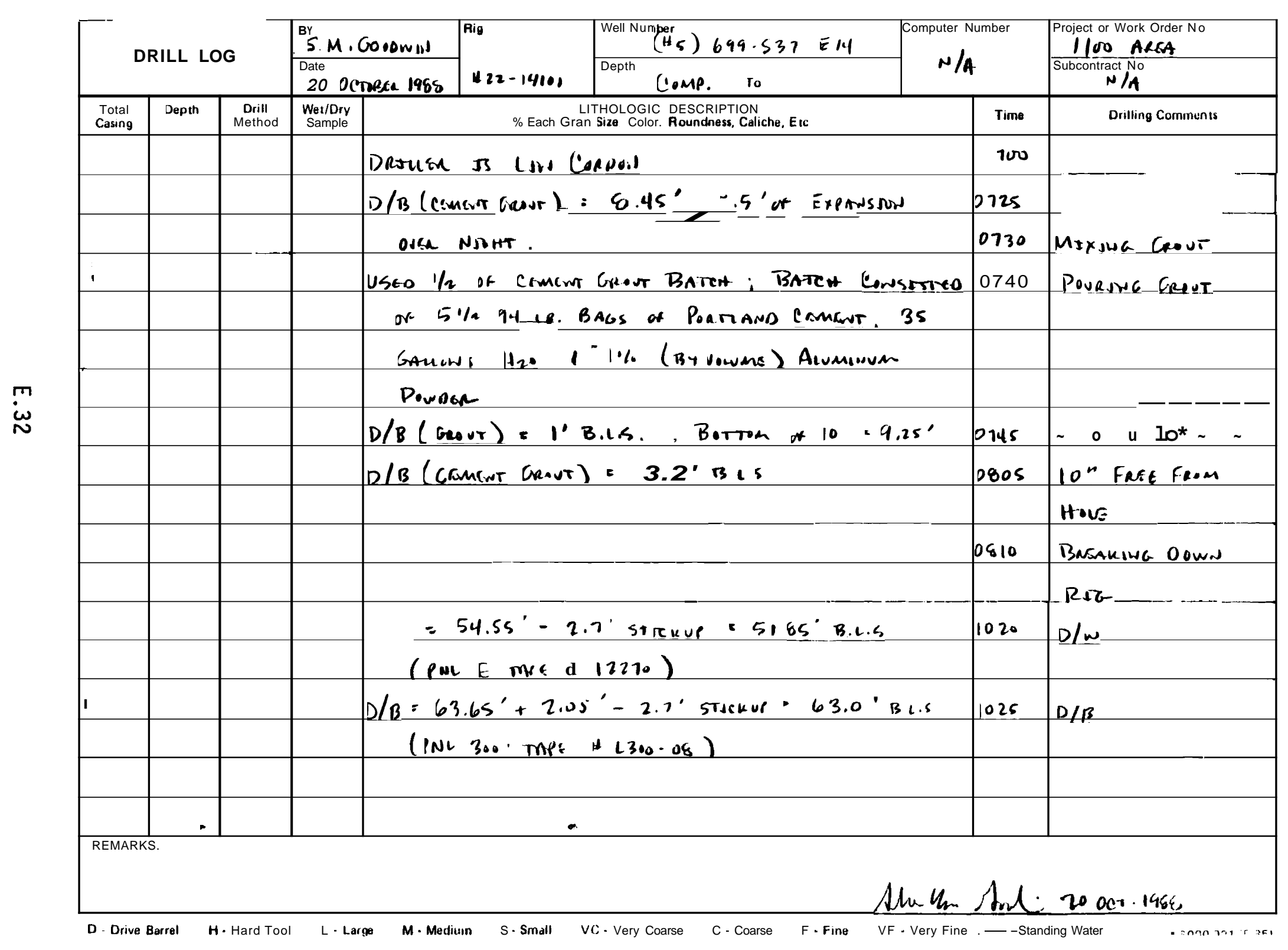




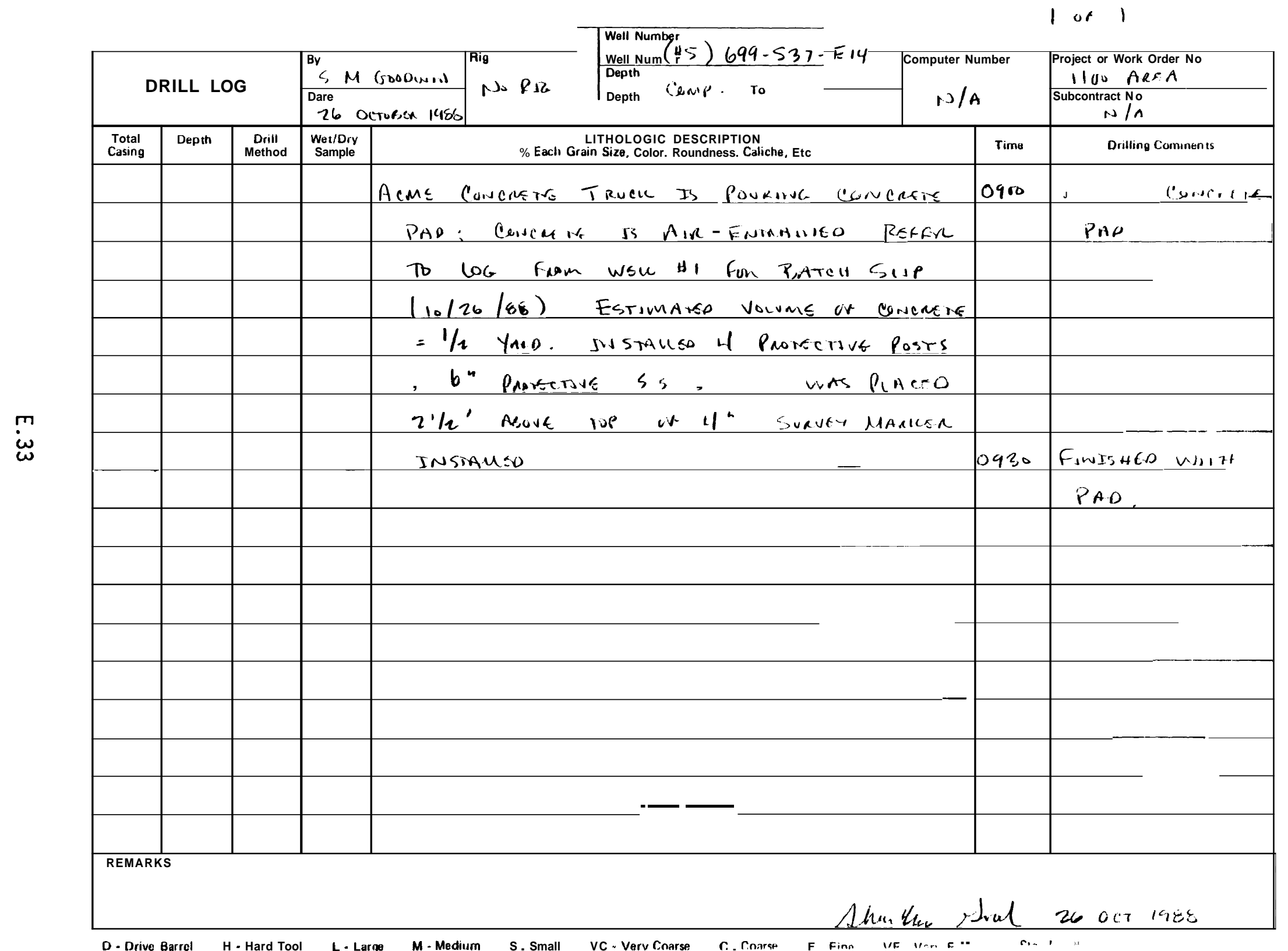


10,1

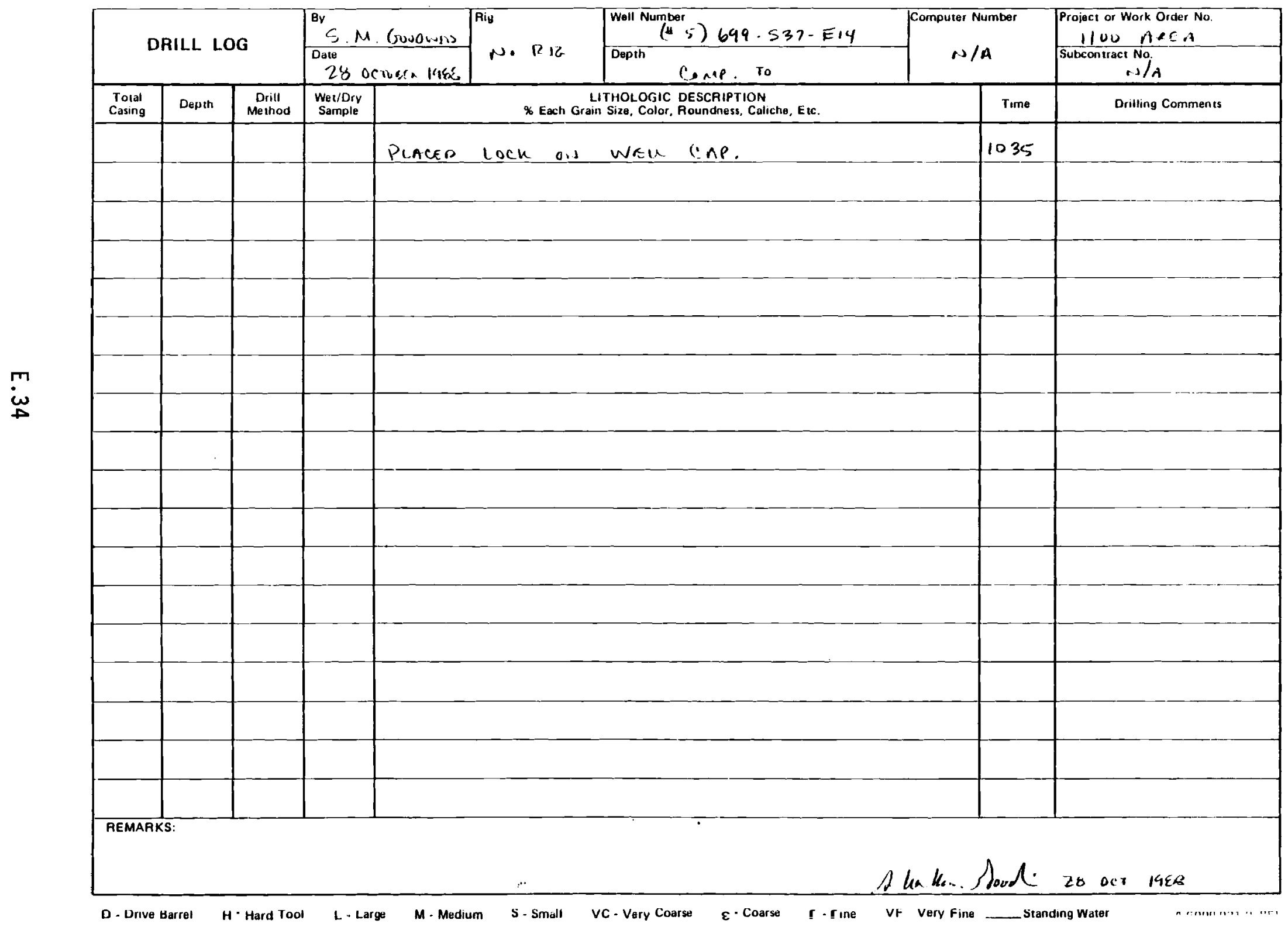


1 of 2

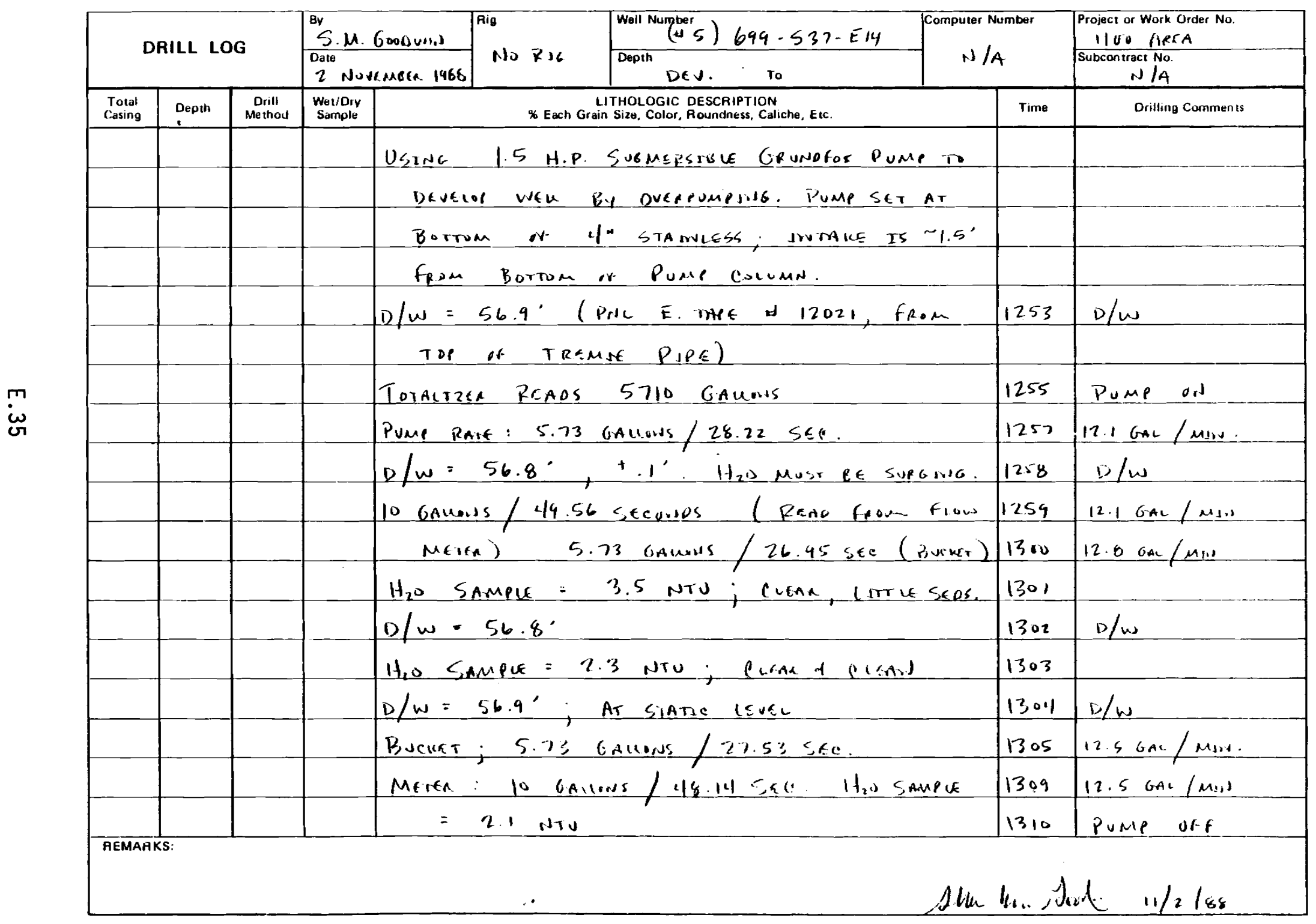

D. Drive Barrel H-Hard Tool L-Large M-Medium S-Small VC-Very Coarse C.Coarse F. Fine VF-Verv Fine - Standing Water 


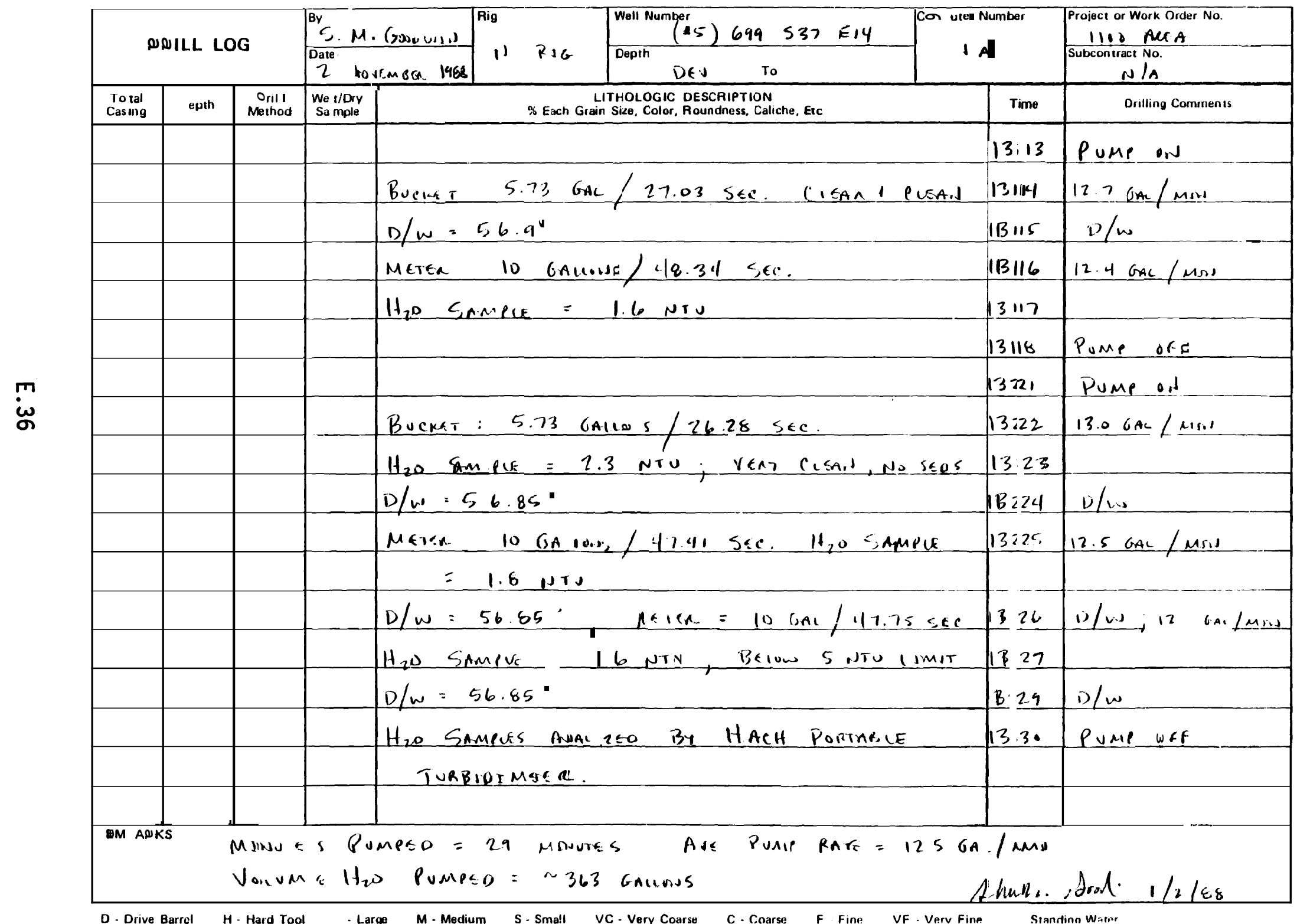


1 or 2

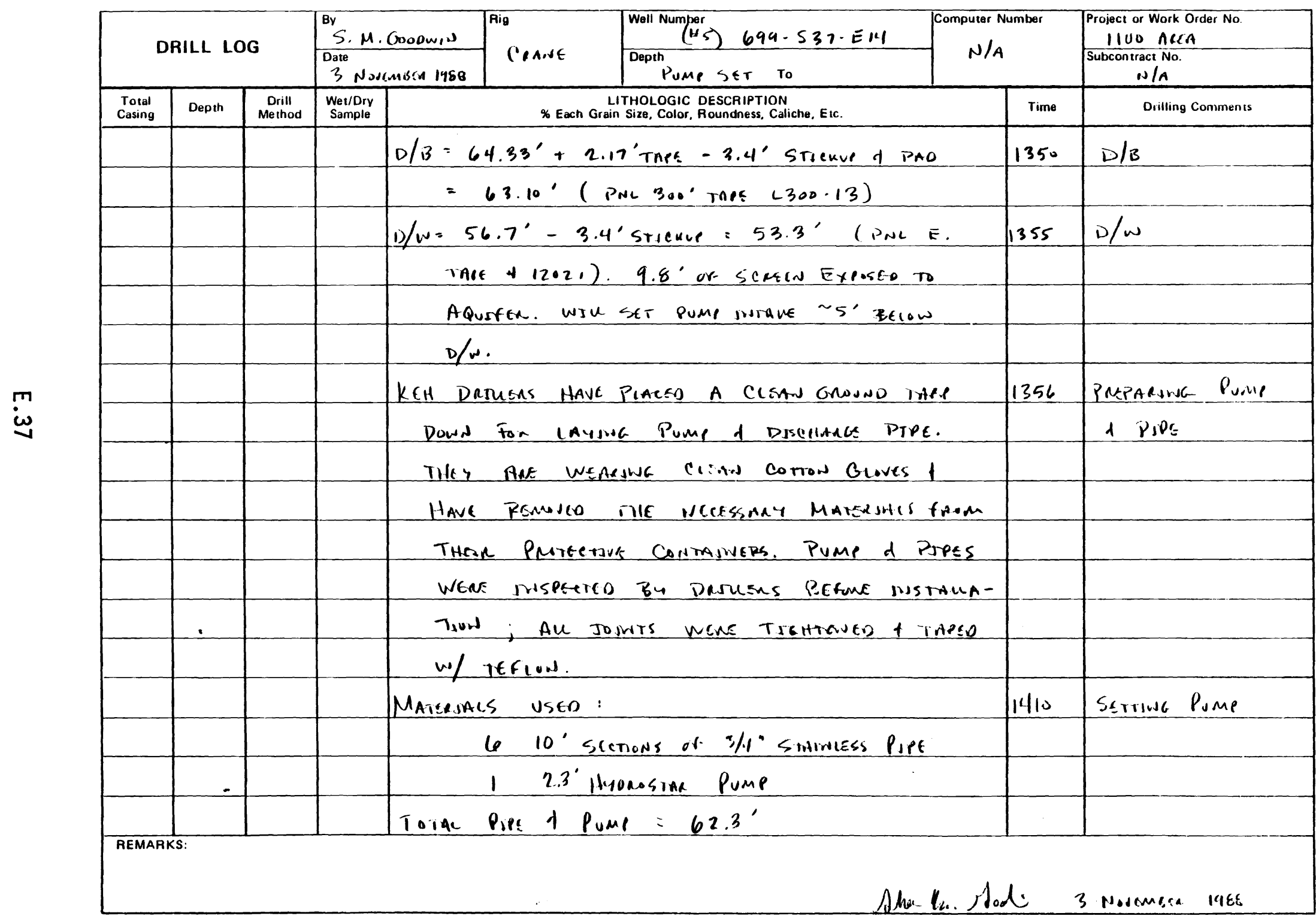




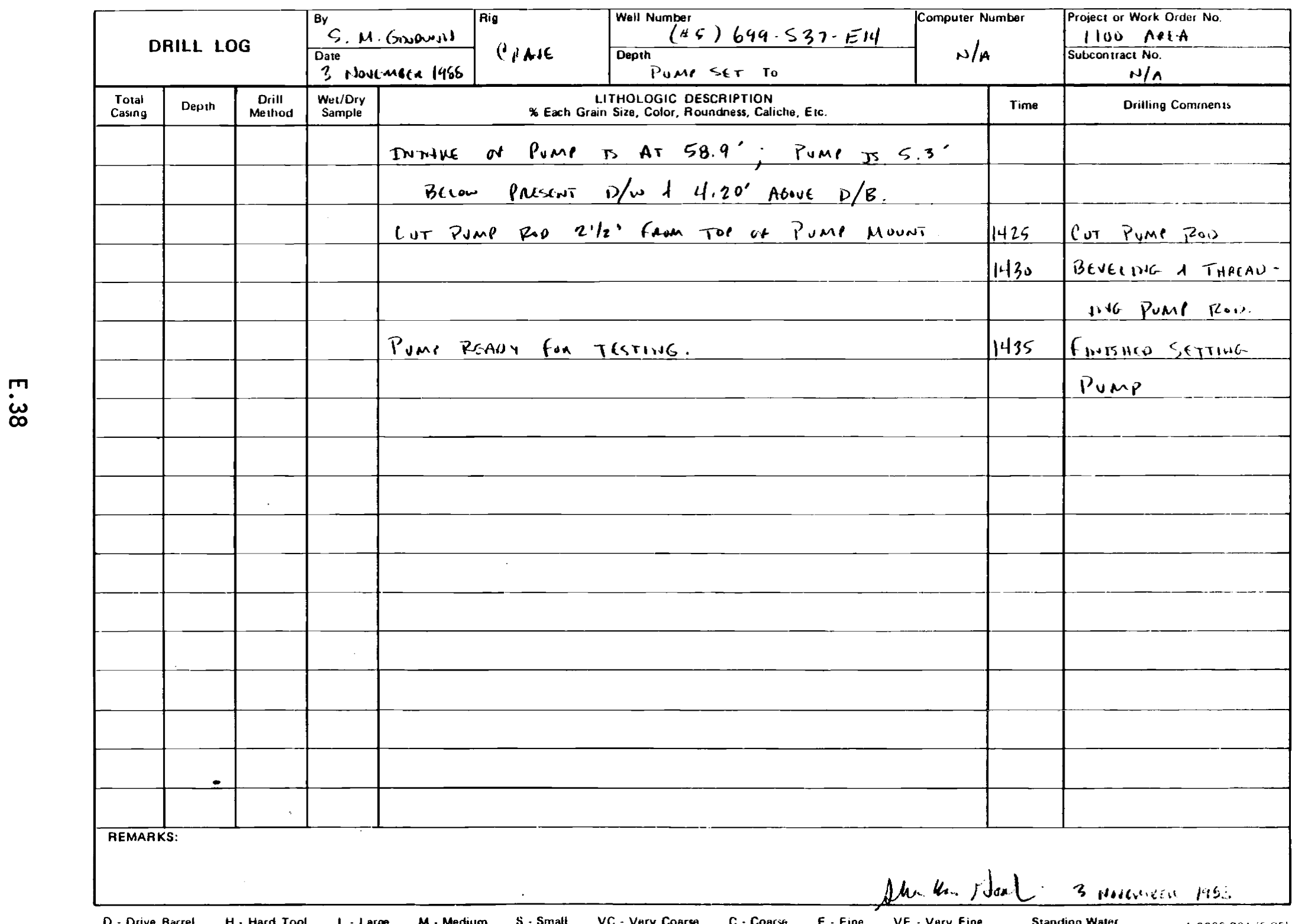

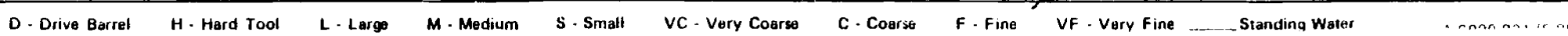


$1 \cdot+1$

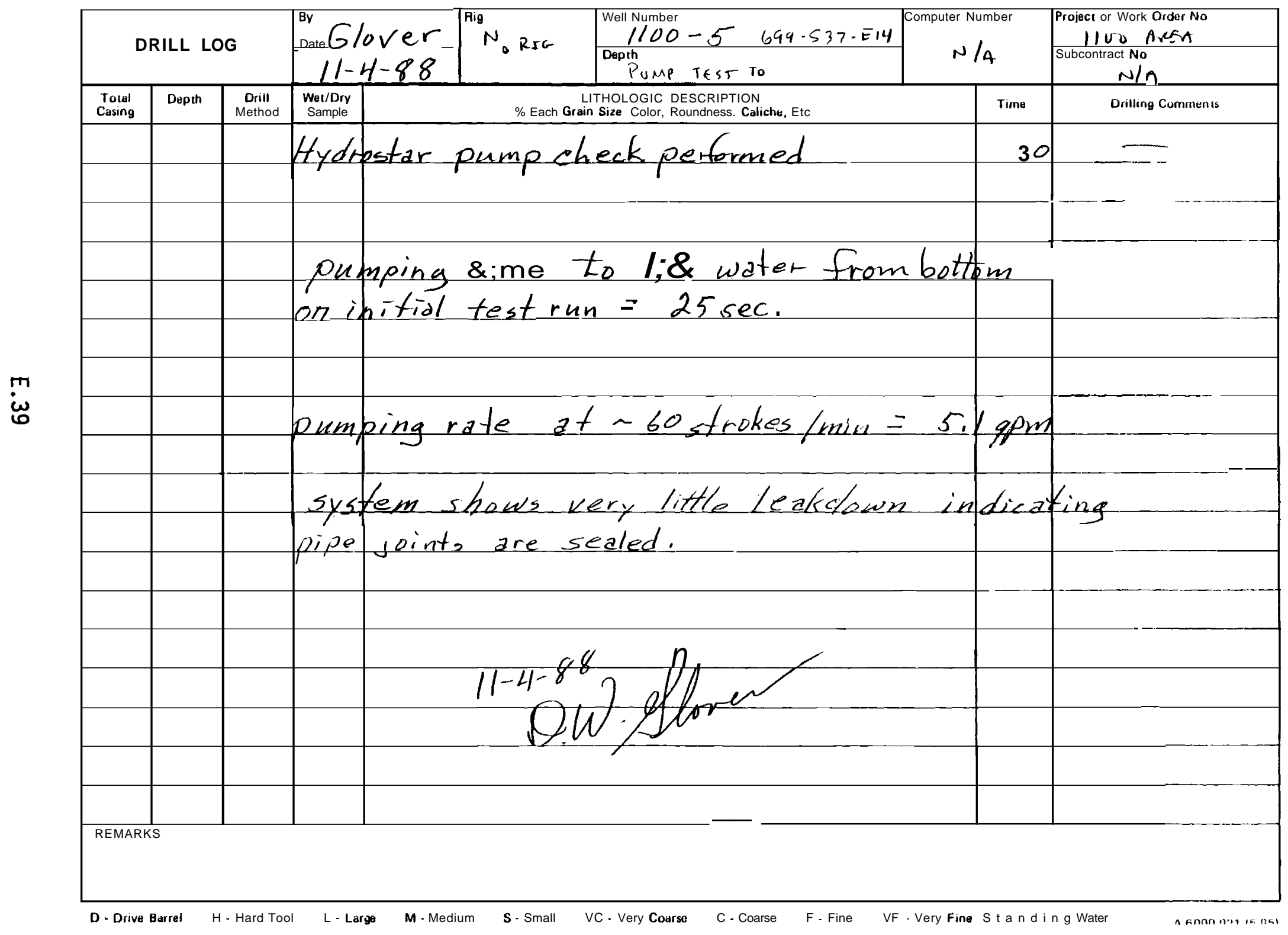




\section{APPENDIX F}

WELL SURVEY DATA 
APPENDIX F

WELL SURVEY DATA

The following pages contain the survey data for the five new wells drilled in the 1100 Area. Kaiser Engineers Hanford performed the surveying. 


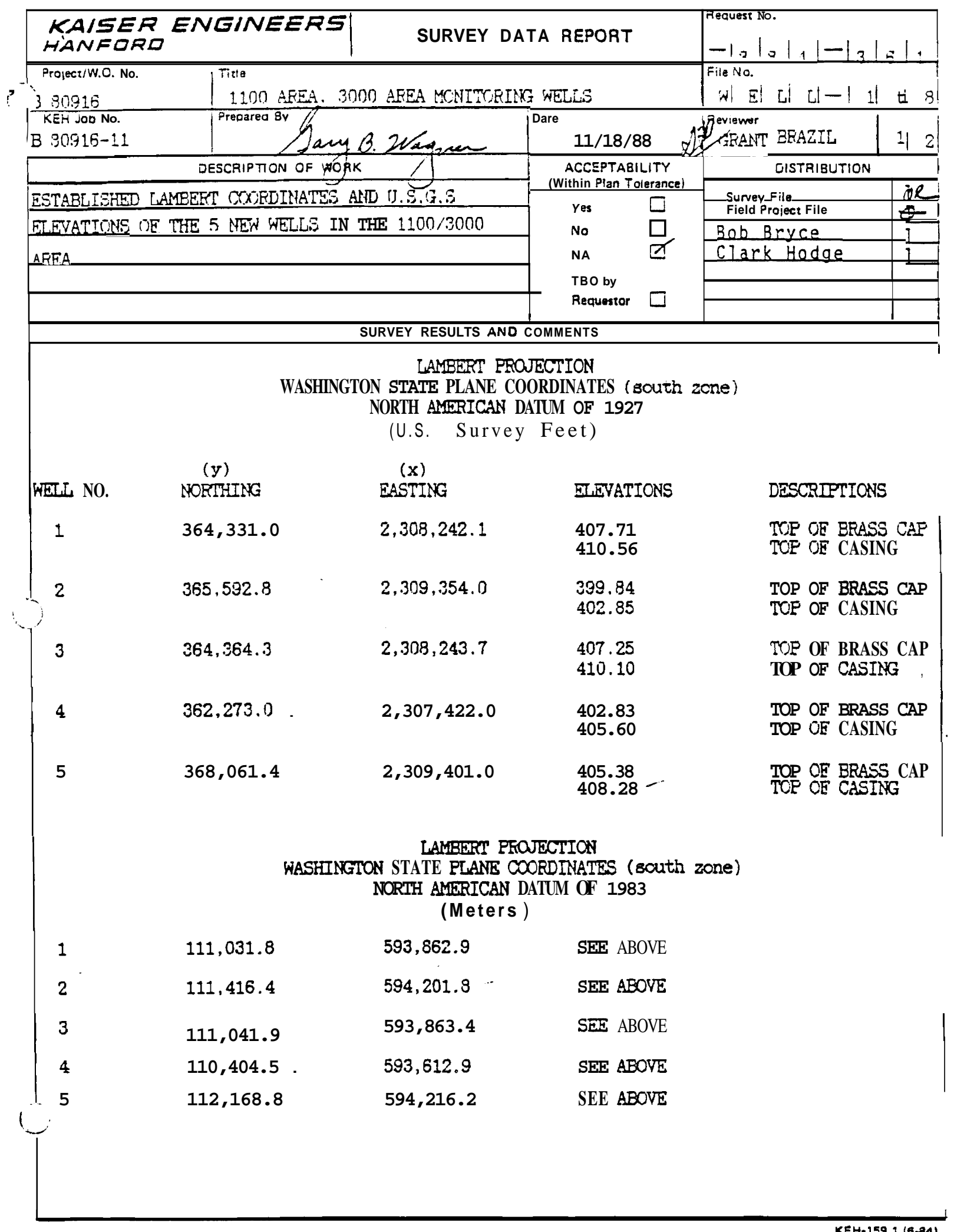

KEH-159. ? (6-94) 


\begin{tabular}{|c|c|c|}
\hline $\begin{array}{l}\text { KAISER ENGINEERS } \\
\text { HANFORD }\end{array}$ & \multicolumn{2}{|c|}{ FIELD SKETCH } \\
\hline $\begin{array}{l}\text { itracnment To or Ooscriorion: } \\
\quad 1100 \text { AREA/3000 AREA }\end{array}$ & MONITORING WELLS & 2 of 2 \\
\hline
\end{tabular}

HANFORO PLANT COCRDINATES OF NEN WELLS IN 1100/3000 AREA

Note: The coominates of the 5 welle listed below were derived from a coominate conversion equation and should be ueed only for the purpose of ldentificartion of the welle and for information only.

6 WELC NOMERR

1

2

3

4

5
SOUTHING

41,004

39,745

40,970

43,060

37,276
EASTING

12.811

13,927

12,813

11,986

13,980 
APPENDIX G

RESULTS OF VOLATILE AND SEMIVOLATILE ORGANIC ANALYSES OF GROUND-WATER SAMPLES 
APPENDIX G

RESULTS OF VOLATILE AND SEMIVOLATILE ORGANIC ANALYSES OF GROUND-WATER SAMPLES

This appendix contains the results of volatile and semivolative organic analyses conducted on samples from the five new monitoring wells in the 1100 Area. Table G.I presents the results of volatile organic analyses of ground-water samples collected November 11 and 14, 1988. These analyses were conducted by Pacific Northwest Laboratory. The pages that follow present the results of the semivolatile organic analyses conducted by U.S. Testing Company, Inc.

TABLE G.1. Results of Volatile Organic Analyses

Concentration(a)

\begin{tabular}{|c|c|c|c|c|c|}
\hline Constituent & Well 1 & Well 2 & Well 3 & Well 4 & Well 5 \\
\hline Methylene Chloride & $<3$ & $<3$ & t3 & $<3$ & $<3$ \\
\hline Chloroform & 0.29 & 0.57 & 0.56 & 0.31 & 5.3 \\
\hline $1,1,1-T$ (b) & t0.02 & $<0.02$ & 0.03 & 0.02 & 0.35 \\
\hline Carbon Tetrachloride & t0.01 & t0.01 & $<0.01$ & t0.01 & $<0.01$ \\
\hline $\operatorname{TCE}(\mathrm{C})$ & 0.03 & $<0.02$ & 0.10 & 0.14 & 0.08 \\
\hline $\mathrm{BDCM}(\mathrm{d})$ & $<0.01$ & 0.03 & t0.01 & $<0.01$ & 0.15 \\
\hline $\operatorname{PCE}(e)$ & 0.12 & t0.01 & 0.22 & 0.02 & 0.13 \\
\hline
\end{tabular}
(a) Concentrations in ppb.
(b) 1,1,1-T = 1,1,1-Trichloroethane.
(c) TCE = Trichloroethylene.
(d) $\mathrm{BDOM}=$ Bromodichloromethane .
(e) $\mathrm{PCE}=$ Perchloroethylene. 


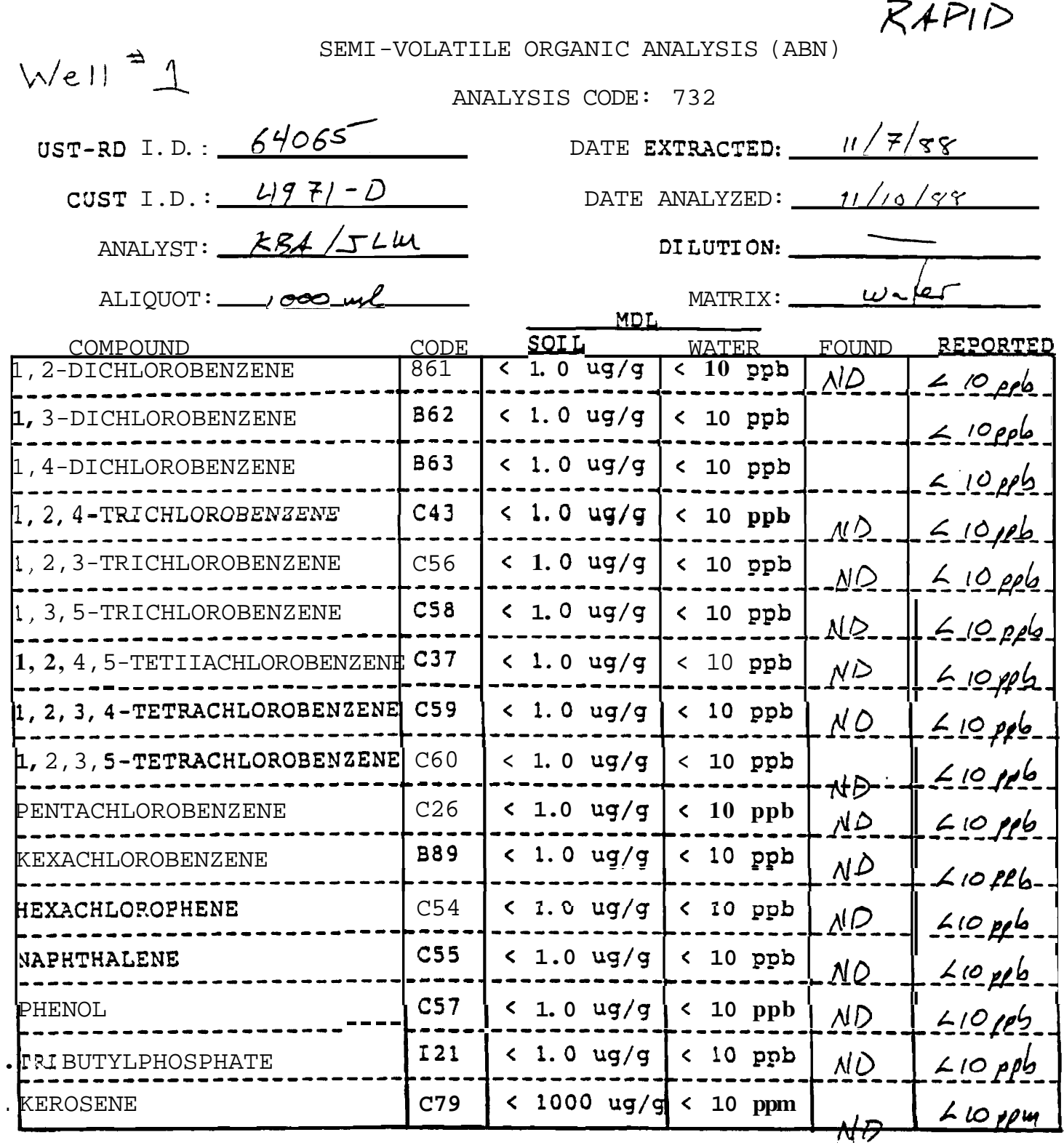

TENTATIVELY IDENTIFIED COMPOUNDS $(>25 \%$ OF NEAREST I.S.,

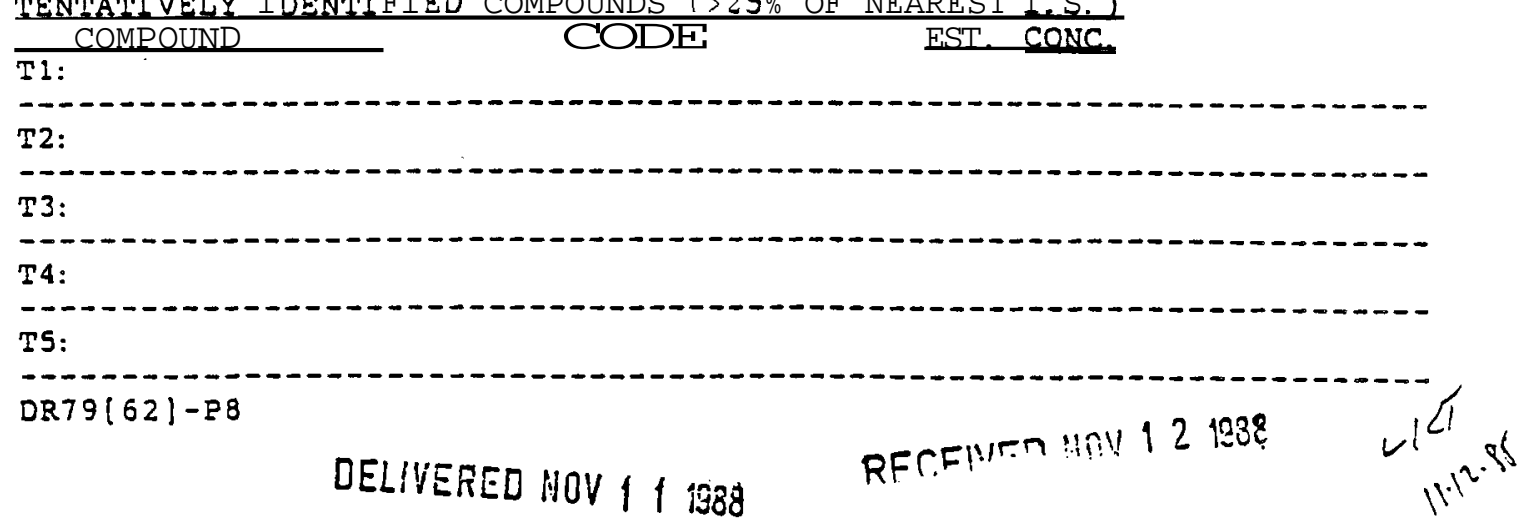




\section{Well $\# 2$ SEMI-VOLATILE ORGANIC ANALYSIS (AEN)}

ANALYSIS CODE: 732

UST-RD I.D. $: \frac{64066}{4972-D}$
CUST I.D.:
ANALYST: $\mathrm{CELCm}$

ANALYS:
DATE EXTRACTED: $11 / 7 / 88$

DATE ANALYZED: $11 / 10 / 5 \%$

DILUTION:

MATRIX:

\begin{tabular}{|c|c|c|c|c|c|}
\hline \multicolumn{2}{|l|}{ ALIQUOT: 1000 and } & MDI & \multicolumn{3}{|c|}{ MATRIX: water } \\
\hline COMPOUND & קס & SOrI & WATER & & \\
\hline 1,2-DICHLOROBENZENE & B61 & $<1.0 \mathrm{ug} / \mathrm{g}$ & $<10 \mathrm{ppb}$ & & \\
\hline 3-DICHLOROBENZENE & $\mathrm{B} 62$ & $\leq 1.0 \mathrm{ug} / \mathrm{g}$ & $<10 \mathrm{ppb}$ & & \\
\hline NF & B63 & $<10$ & 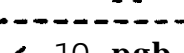 & & \\
\hline $14-D \perp C \Pi \omega$ & Bos & $\therefore--\infty$ & $<10$ pgo & & \\
\hline 1, 2,4-TRICHLOROBENZENE & C43 & $\mathrm{C} 1.0 \mathrm{ug} / \mathrm{g}$ & C $10 \mathbf{p p b}$ & & \\
\hline $1,2,3$-TRI CHLOROBENZENE & C56 & $<1.0 \mathrm{ug} / \mathrm{g}$ & $<10 \mathrm{ppb}$ & & \\
\hline $1,3,5$ TRICHLOROBENZENE & C58 & $<1.0 \mathrm{ug} / \mathrm{g}$ & $<10$ ppb & & \\
\hline $1,2,4,5$-TETRACHLOROBENZ & C37 & $<1.0 \mathrm{ug} / \mathrm{g}$ & $<10 \mathrm{ppb}$ & & \\
\hline $1,2,3,4$-TETRACHLOROBENZ & C59 & $<1.0 \mathrm{ug} / \mathrm{g}$ & $<10$ ppb & & \\
\hline$[1,2,3,5$-TETRACHLOROBENZENE & $\mathrm{C} 60$ & $<1.0 \mathrm{ug} / \mathrm{g}$ & $<10$ ppb & & \\
\hline PENTACHLOROBENZENE & $\mathrm{C} 26$ & $1.0 \mathrm{ug} / \mathrm{g}$ & $<.10 \mathrm{ppb}$ & & \\
\hline HEXACHLOROBENZENE & B89 & $<1.0 \mathrm{ug} / \mathrm{g}$ & $<10$ ppb & & \\
\hline & & & & & $\leq 10-4$ \\
\hline HEXACHLOROPHENE & C5 4 & $<1.0 \mathrm{ug} / \mathrm{g}$ & $<10$ ppb & & \\
\hline NAP HTHALENE & C55 & $<1.0 \mathrm{ug} / \mathrm{g}$ & $<10$ ppb & & \\
\hline IENOL & C57 & $<1.0 \mathrm{ug} / \mathrm{g}$ & & & \\
\hline & & & & & 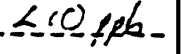 \\
\hline TRI BUTYLPHOSPHATE & I 21 & $<1.0 \mathrm{ug} / \mathrm{g}$ & $<10$ ppb & & 10 \\
\hline KEROSENE & C79 & $<1000 \mathrm{ug} / \mathrm{g}$ & $<10 \mathbf{p p m}$ & & $\angle 10$ \\
\hline
\end{tabular}
$\frac{\text { TENTATIVELY IDENTIFISD COMPOUNDS (225*OF NEAREST } T \text {. S) }}{\text { COMPOUND }}$

T1:

$--$

T2:

T3:

19:

T4:

T5:

DR79[62]-PB

OELIVERED NOV \& । 1988 
Well $\# 3$

SこMI-VOLATILE ORGANIC ANALYSIS (ABN)

RAPID

ANALYSIS CODE: 732

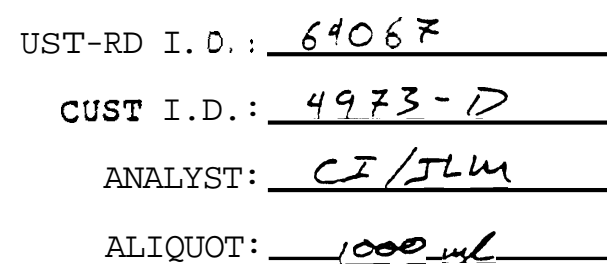

DATE EXTRACTED: $11 / 7 / 88$

DATE ANALYZED: $11 / 10 / 8$

\begin{tabular}{|c|}
\hline BENZENE \\
\hline CHLOROBENZENE \\
\hline 1,4 -DICHLOROBENZENE \\
\hline ROBENZENE \\
\hline TRICHLOROBENZENE \\
\hline HLOROBENZENE \\
\hline
\end{tabular}

CODE

SOII

DI IUTION:

MATRIX:

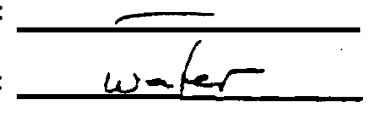

MDI WATER FOUND REPORTED

$1,2,4,5$-TETRACHLOROBENZENE C3

$1,2,3,4$-TETRACHLOROBENZENE C59

$1,2,3,5$-TETRACHLOROBENZENA C60

EENTACHLOROBENZENE

HEXACHLOROBENZENE

(EXACHLORÓHENE

-

PHENOL

che

ERIBUTYLPHOSPHATE

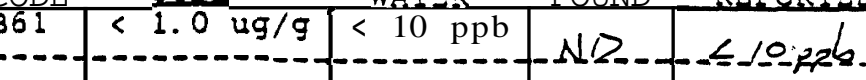
B62<1.0 ug/g $<10 \mathrm{ppb}$ $363<1.0 \mathrm{ug} / \mathrm{g}<10 \mathrm{0g}$

-

KEROSENE

\section{TENTATIVELY IDENTTEIED COMPOUNDS (25\% OF NEAREST I.S.) COMPOUND CODE EST CONC.}

T1:

$\mathrm{T} 2$ :

T3:

T4:

T5.

DR79[52]-P8 


\section{Well ${ }^{\# 4}$}

SEMI-VOLATILE ORGANIC ANALYSIS (ABN)

ANALYSIS CODE: 732
UST-RD I. D. : $-6406 \%$ CUST I.D.: $4974-D$

.ANALYST: 1

ALIQUOT: 1000 me
DATE EXTRACTED: DATE ANALYZED:

DILUTION : -- MATRIX: mive MATRIX: CODE SOIL WATER FOUND RERORTEO

\begin{tabular}{|c|c|c|c|c|c|}
\hline COMPOUND & CODE & SOIL & warea & FOUND & \\
\hline 1. 2-DICHLOROBENZENE & 861 & $<1.0 u g / g$ & $<10$ pob & & \\
\hline $1,3-D I C H L O R O B E N Z E N E$ & B62 & $<1.0 \mathrm{ug} / \mathrm{g}$ & $<10 \mathrm{ppb}$ & & \\
\hline $1,4-D I C H L O R O B E N Z E N E$ & 863 & $<1.0 \mathrm{ug} / \mathrm{g}$ & c 10 ppb & & \\
\hline 1. 2, 4-TRI CHLOROBENZENE & $\mathrm{C} 43$ & $<1.0$ ugi $;$ & C $10 \mathrm{ppb}$ & & \\
\hline $1,2,3$-TRICHLOROBENZENE & C56 & $<1.0 \mathrm{ug} / \mathrm{g}$ & C $10 \mathrm{ppb}$ & & \\
\hline $1,3,5-T R I C H L O R O B E N Z E N E$ & C58 & $<1.0 \mathrm{ug} / \mathrm{g}$ & $<10 \mathrm{ppb}$ & & \\
\hline $1,2,4,5$-TETRACHLOROBENZENE & C37 & $<1.0 \mathrm{ug} / \mathrm{g}$ & $<10 \mathrm{ppb}$ & & \\
\hline $1,2,3,4-\mathrm{TETRACHLOROBENZENE}$ & $\operatorname{csg}$ & $\mathrm{C}-1.0 \begin{array}{cc}0 & \mathrm{ug} / \mathrm{g}\end{array}$ & $<10$ ppb & & \\
\hline $1,2,3,5$-TETRACHLOROBENZENE & $\mathrm{C} 60$ & $<1.0 \mathrm{ug} / \mathrm{g}$ & $<10 \mathrm{ppb}$ & & \\
\hline PENTACHLOROBENZENE & $\mathrm{C} 26$ & $<1.0 \mathrm{ug} / \mathrm{g}$ & $<10 \mathrm{ppb}$ & & \\
\hline HEXACHLOROBENZENE & $\mathrm{B} 89$ & C $1.0 \mathrm{ug} / \mathrm{g}$ & C 10 ppb & & \\
\hline HEXACHLOROP HENE & $\mathrm{Cs} 4$ & $<1.0 \mathrm{ug} / \mathrm{g}$ & $<10 \mathrm{pgb}$ & & \\
\hline NAPHTHALENE & C55 & $<1.0, \mathrm{ug} / \mathrm{g}$ & $<10 \mathrm{ppb}$ & & \\
\hline 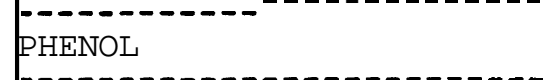 & $\operatorname{cs} 7$ & < $1.0 \mathrm{ug} / \mathrm{g}$ & $<10 \mathrm{p}$ & & \\
\hline IRIBUTYLPHOSPHATE & 121 & $<1.0 \mathrm{ug} / \mathrm{g}$ & $<10$ ppb & & \\
\hline KEROSENE & C79 & $<1000 \mathrm{ug} / 9$ & $<10 \mathrm{ppm}$ & ND & $\angle 10$ \\
\hline
\end{tabular}

TENTATIVELY IDENTIFIED COMPOUNDS (>25\% OF NEAREST I.S.)

COMPOUND
T1:
T2:
T3:
T4:
T5:
DR7 $9[62]-\mathrm{CODE}$

DELIVERED NOY $\mid$ ! 1988 
We $11: 5$ SEMI-VOLATILE ORGANIC ANALYSIS (ABN)

$R A P I D$

ANALYSIS CODE: $\mathbf{7 3 2}$

UST-RD I. D. : 64069

DATE EXTRACTED: $11 / 7 / 88$

CUST I.D. : $4975-0$

DATE ANALYZED: $11 / 10 / 88$

ANALYST: $c I / J L U$

ALIQUOT: _, _o us

DILUTION :

MATRIX:

ser

COMPOUND

\begin{tabular}{|c|c|c|c|c|c|}
\hline COMPOUND_ & Eمع & SOI & WTATER & FOUNO & $\mathrm{RSPCF}$ \\
\hline $1,2-\mathrm{DICHLOROBENZENE}$ & 861 & $<1.0 \mathrm{ug} / \mathrm{g}$ & $<10 \mathrm{ppb}$ & & \\
\hline $1,3-D I C H L O R O B E N Z E N E$ & B62 & c $1.0 \mathrm{ug} / \mathrm{g}$ & c $10 \mathrm{ppb}$ & & \\
\hline 1, 4-DI CHLOROBENZENE & B63 & $\leq 1.0 \mathrm{ug} / \mathrm{g}$ & $<10$ ppb & & \\
\hline $1,2,4$-TRICHLOROBENZENE & $\mathrm{C} 43$ & $<1.0 \mathrm{ug} / \mathrm{g}$ & c 10 ppb & & \\
\hline $1,2,3$-TRI CHLOROBENZENE & C56 & $<1.0 \mathrm{ug} / \mathrm{g}$ & $<10 \mathrm{ppb}$ & & \\
\hline $1,3,5-T R I C H L O R O B E N Z E N E$ & C58 & $<1.0 \mathrm{ug} / \mathrm{g}$ & c 10 ppb & & \\
\hline$[1,2,4,5$-TETRACHLOROBENZENH & C37 & C $1.0 \mathrm{ug} / \mathrm{g}$ & $<10 \mathrm{ppb}$ & & \\
\hline $1,2,3,4$-TETUCHLOROBENZENE & $\operatorname{c5g}$ & C $1.0 \mathrm{ug} / \mathrm{g}$ & $<10$ ppb & & \\
\hline $1,2,3,5$-TETRACHLOROBEN Z ENE & $\mathrm{c} 60$ & $<1.0 \mathrm{ug} / \mathrm{g}$ & $<10 \mathrm{ppb}$ & & \\
\hline PENTACHLOROBENZENE & $C 26$ & $<1.0 \mathrm{ug} / \mathrm{g}$ & $<10 \mathrm{ppb}$ & & \\
\hline HEXACHLOROBENZENE & 889 & $<1.0 \mathrm{ug} / \mathrm{g}$ & c 10 ppb & & \\
\hline IEXACHLOROPHENE & C5 4 & $<1.0 \mathrm{ug} / \mathrm{g}$ & c 10 ppb & 36 & \\
\hline 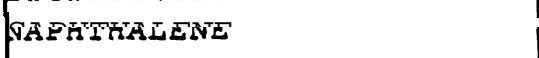 & C55 & $<2.0 \mathrm{ug} / \mathrm{g}$ & $<10$ ppb & & \\
\hline PHENOL & C57 & $<1.0 \mathrm{ug} / \mathrm{g}$ & c 10 ppb & & \\
\hline IRI BUTYLPHOSPHATE & I21 & $<1.0 \mathrm{ug} / \mathrm{g}$ & $<10 \mathrm{ppb}$ & $\mathrm{Ni}$ & \\
\hline KEROSENE & C79 & $<1000 \mathrm{ug} / \mathrm{g}$ & < 10 ppm & ND & $\angle 10$ \\
\hline
\end{tabular}

$\begin{array}{ccc}\text { TENTATTVETY IDENTIETED COMPOUNDS }(>25 \% & \text { OF } & \text { NEAREST I.S. ) } \\ \text { COMPOUND } & \text { CODE } & \text { EST. CONC. }\end{array}$

T1:

T2:

12:

T3:

$\mathrm{T} 4:$

$--$

T5:

DR79[62]-P8

DELIVERED NOV $\mid \uparrow 1988$

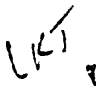

11.9 
APPENDIX H

RESULTS OF GROUND-WATER ANALYSES FROM NEW MONITORING WELLS 
RESULTS OF GROUND-WATER ANALYSES FROM NEW MONITORING WELLS

This appendix contains the results of chemical and volatile organic analyses conducted on ground-water samples collected November 7, 1988, from five new monitoring wells in the 1100 Area.

\section{TABLE H.1. Results of Chemical Analyses Conducted by U.S. Testing Company, Inc.}

\begin{tabular}{|c|c|c|c|c|c|}
\hline \multirow[b]{2}{*}{ Constituent } & \multicolumn{5}{|c|}{ Concentration (a) } \\
\hline & Uell 1 & Well 2 & Well 3 & Well 4 & Uell 5 \\
\hline Jitrate & $7.8 \mathrm{ppm}$ & $1 \mathrm{ppm}$ & $4 \mathrm{ppm}$ & $21.2 \mathrm{ppm}$ & $3 \mathrm{ppm}$ \\
\hline Chloride & $4.9 \mathrm{ppm}$ & $0.9 \mathrm{ppm}$ & $8.1 \mathrm{ppm}$ & $43 \mathrm{ppm}$ & $2.1 \mathrm{ppm}$ \\
\hline Fluoride & $<0.5 \mathrm{ppm}$ & $<0.5 \mathrm{ppm}$ & $<0.5 \mathrm{ppm}$ & $<0.5 \mathrm{ppm}$ & $<0.5$ pom \\
\hline Sulfate & $11.6 \mathrm{ppm}$ & $11.9 \mathrm{ppm}$ & $11.5 \mathrm{ppm}$ & 26.9 ppm & $11.5 \mathrm{ppm}$ \\
\hline $\begin{array}{l}\text { Phosphate } \\
\text { TOX }\end{array}$ & $\begin{array}{l}<1 \mathrm{ppm} \\
<13\end{array}$ & $\begin{array}{l}<1 \mathrm{ppm} \\
44\end{array}$ & $\begin{array}{l}<1 \mathrm{ppm} \\
<8.0\end{array}$ & $\begin{array}{l}<1 \mathrm{ppm} \\
59\end{array}$ & $\begin{array}{l}<1 \mathrm{ppm} \\
61\end{array}$ \\
\hline TOC & $<700$ & $<600$ & $<700$ & $1.2 \mathrm{ppm}$ & $<600$ \\
\hline TC & $32.6 \mathrm{pprn}$ & $16.9 \mathrm{ppm}$ & $47.8 \mathrm{ppm}$ & $53.6 \mathrm{ppm}$ & 20.2 ppm \\
\hline $\mathrm{pH}$ & 7.6 & 7.6 & 7.5 & 7.6 & 7.8 \\
\hline Alkalinity & $142 \mathrm{ppm}$ & 73 ррп & 214 ppm & 234 ppm & 89.5 ppm \\
\hline
\end{tabular}

Metals Analyzed by the Inductively Coupled Plasma Method

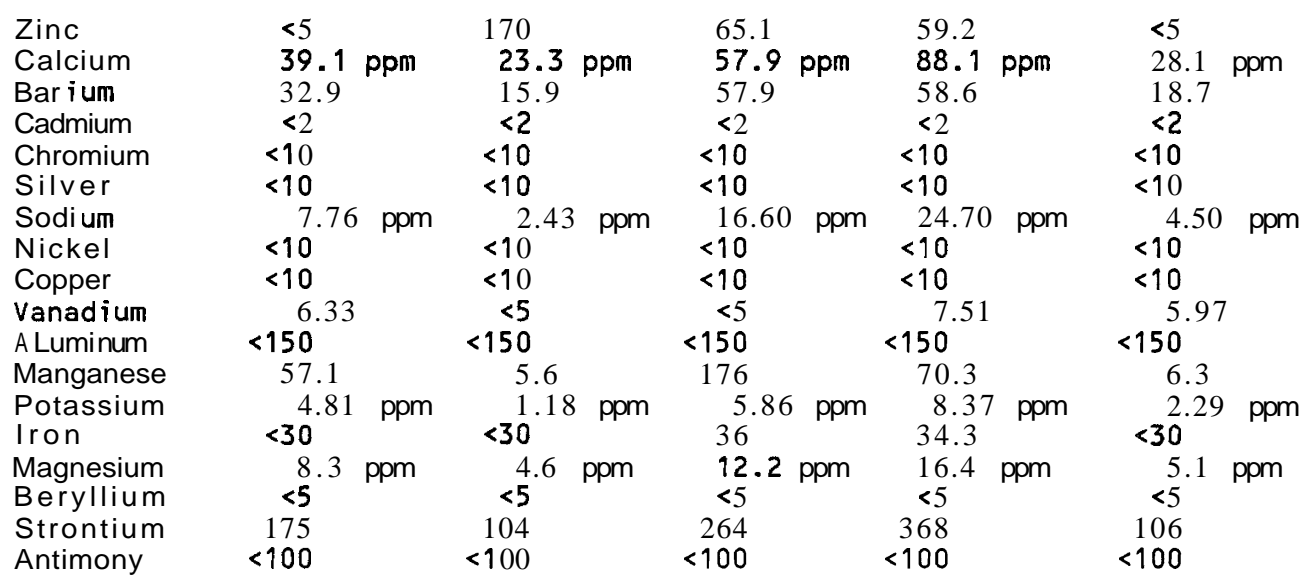

Metals Analyzed by the Gas Furnace/Atomic Absorption Method

\begin{tabular}{|c|c|c|c|c|c|}
\hline Arsenic & $<5$ & $<5$ & $<5$ & $<5$ & $<5$ \\
\hline Selenium & $<5$ & $<5$ & $<5$ & $<5$ & $<5$ \\
\hline Lead & $<5$ & $<5$ & $<5$ & $<5$ & $<5$ \\
\hline Thallium & $<5$ & $<5$ & $<5$ & $<5$ & $<5$ \\
\hline Mercury & $<0.1$ & $<0.1$ & $<0.1$ & $<0.1$ & $<0.1$ \\
\hline
\end{tabular}


TABLE H.1. (contd)

\begin{tabular}{|c|c|c|c|c|c|}
\hline & & & Atration & & \\
\hline Constituent & Well 1 & Well 2 & Well 3 & Well 4 & Well 5 \\
\hline Pesticides & & & & & \\
\hline Endr in & $<0.1$ & $<0.1$ & $<0.1$ & $<0.1$ & $<0.1$ \\
\hline Methoxychlor & $<3$ & $<3$ & $<3$ & $<3$ & $<3$ \\
\hline Toxaphene & $<1.0$ & $<1.0$ & $<1.0$ & $<1.0$ & $<9.0$ \\
\hline Alpha BHC & $<0.1$ & $<0.1$ & $<0.1$ & $<0.1$ & $<0.1$ \\
\hline Beta BHC & $<0.1$ & $<0.1$ & $<0.1$ & $<0.1$ & $<0.1$ \\
\hline Gamma BHC & $<0.1$ & $<0.1$ & $<0.1$ & $<0.1$ & $<0.1$ \\
\hline Delta BHC & $<0.1$ & $<0.1$ & $<0.1$ & $<0.1$ & $<0.1$ \\
\hline Herbicides & $<2.0$ & $<2.0$ & $<2.0$ & $<2.0$ & $<2.0$ \\
\hline Ethylene & & & & & \\
\hline Glycol & $<10$ ppm & $<10 \mathrm{ppm}$ & $<10 \mathrm{ppm}$ & $<10 \mathrm{ppm}$ & $<10 \mathrm{ppm}$ \\
\hline$\underline{\mathrm{PCB}}$ & $<1.0$ & $<1.0$ & $<1.0$ & $<1.0$ & $<1.0$ \\
\hline VOAS & & & & & \\
\hline Carbon Tetra- & & & & & \\
\hline $\begin{array}{l}\text { chloride } \\
\text { Methone }\end{array}$ & $<5$ & $<5$ & $<5$ & $<5$ & $<5$ \\
\hline Methone (D) & $<10$ & $<10$ & $<10$ & $<10$ & $<10$ \\
\hline $1,1,1-T$ (c) & $<5$ & $<5$ & $<5$ & $<5$ & $<5$ \\
\hline $1,1,2-T^{(d)}$ & $<5$ & $<5$ & $<5$ & $<5$ & $<5$ \\
\hline$T C E(e)$ & $<5$ & $<5$ & $<5$ & $<5$ & $<5$ \\
\hline$P C E^{(f)}$ & $<5$ & $<5$ & $<5$ & $<5$ & $<5$ \\
\hline Opxylen (g) & $<5$ & $<5$ & $<5$ & $<5$ & $<5$ \\
\hline Chloroform & $<5$ & $<5$ & $<5$ & $<5$ & $<5$ \\
\hline $\begin{array}{l}\text { Methylene. } \\
\text { chloride }\end{array}$ & $12^{(h)}$ & $72^{(h)}$ & $<10$ & $78^{(h)}$ & $<10$ \\
\hline $\begin{array}{l}M-x y l e(1) \\
\text { Hexone }(j)\end{array}$ & $<5$ & $<5$ & $<5$ & $<5$ & $<5$ \\
\hline nexon & $<10$ & $<10$ & $<10$ & $<10$ & $<10$ \\
\hline$A B N s$ & $<$ detect & $<$ detect & $<$ detect & $<$ detect & $<$ detect \\
\hline
\end{tabular}

(a) Concentrations are in ppb unless otherwise stated.

(b) Methone $=$ Methylethyl ketone.

(c) $1,1,1-T=1,1,1$ Trichloroethane.

(d) $1,1,2-T=1,1,2$ Trichloroethane.

(e) $T C E=$ Trichloroethylene.

(f) PCE = Perchloroethene.

(g) Opxylen = Ortha para xylene.

(h) Methylene chloride was below detection for samples collected November 11 and 14, 1988, and analyzed by PNL, indicating samples collected November 7, 1988, and analyzed by U.S. Testing and PNL were contaminated with methylene chloride during sample collection process.

(i) M-xyle = Meta xylene.

(j) Hexone = Methyl isobutyl ketone. 
TABLE H.2. Results of Volatile Organic Analyses Conducted by Pacific Northwest Laboratory

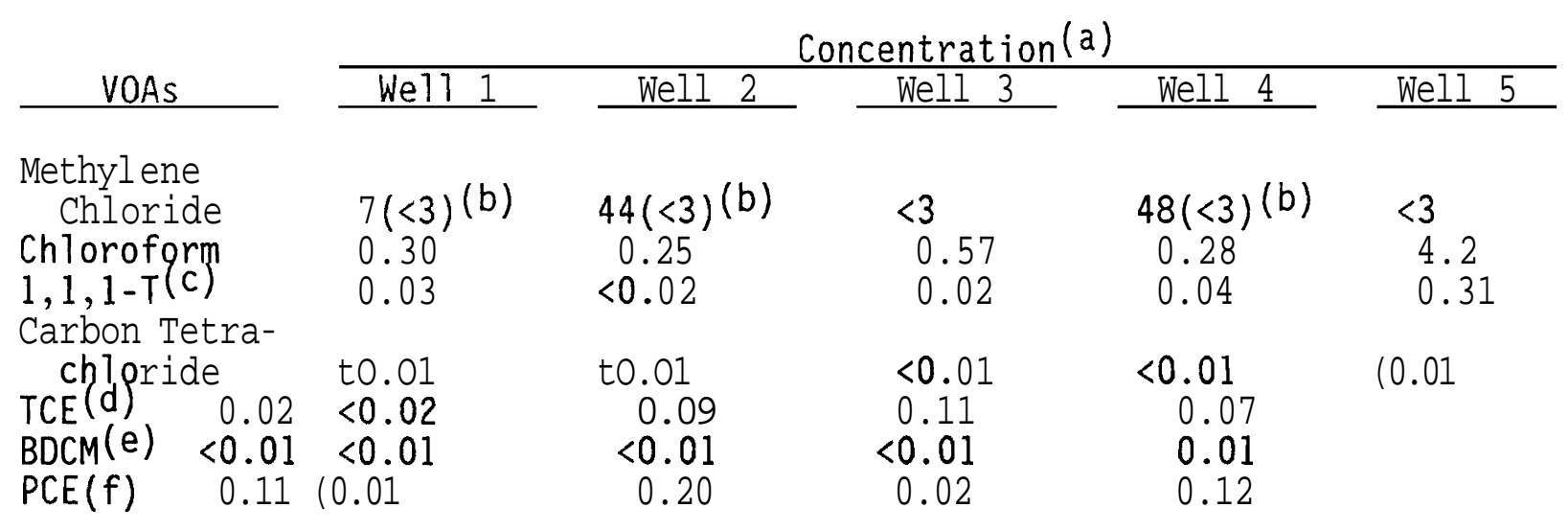

(a) Concentrations are in ppb unless otherwise stated.

(b) Methylene chloride was below detection for samples collected November 11 and 14, 1988, and analyzed by PNL, indicating samples collected November 7, 1988, and analyzed by U.S. Testing and PNL were contaminated with methylene chloride during sample collection process.

(c) $1,1,1-\mathrm{T}=1,1,1-$ Trichloroethane.

(d) TCE = Trichtoroethylene.

(e) $\mathrm{BDCM}=$ Brornodichloromethane.

(f) $\quad$ PCE $=$ Perchloroethylene.

Semivolatile organic constituents (ABNs) have varying detection limits. The raw data report for one sample is included in Appendix $G$ to indicate the complete list of analyses and the detection limits. 
PNL -6824

UC - 11,41

\section{DISTRIBUTION}

No. of

$\underline{\text { Copies }}$

OFFSITE

2 DOE/Office of Scientific and Technical Information

QNSITE

2 DOE Richland Operations Office

E. A. Bracken

M. L. Tiernan

6 Westinghouse Hanford Company

G. Carpenter

K. R. Fecht

R. L. Jackson

K. N. Jordan

R. G. McCain

L. C. Swanson
No. of

Copies

27 Pacific Northwest Laboratory

R. W. Bryce (5)

J. W. Falco

S. M. Goodwin

R. E. Gephart

R. H. Gray (2)

J. M. Hales

P. C. Hays

G. V. Last

T. L. Liikala

R. E. Lundgren

P. J. Mitchell

R. L. Skaggs

R. M. Smith

J. A. Stottlemyre

S. S. Tee1

R. E. Wildung

Publishing Coordination

Technical Report Files (5) 